\title{
E.Y. DAWSON
}

JEAN CHALON

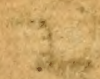

\section{LISTE}

:

DES

ALGUES MARINES

OBSERVÉES JUSQU'A CE JOUR

ENTRE L'EMBOUCHURE DE L'ESCAUT ET LA COROGNE INCL. ILES ANGLO-NORMANDES

QK

572.9

C43

1905

BOT

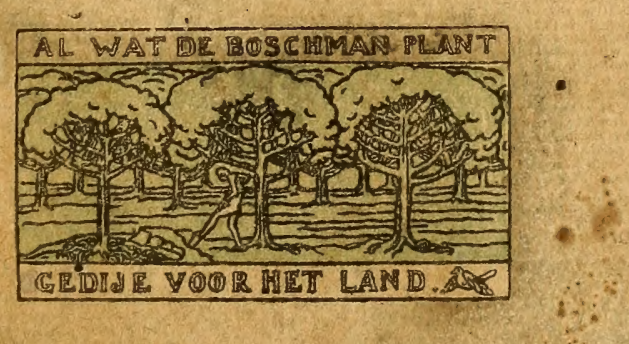

ANVERS

IMPRIMERIE J,-E. BUSCHMANN

REMPART DE LA PORTE DU hHIN

FÉVRIER 1905. 


\section{Liste des Algues marines}

observées jusqu'à ce jour entre l'embouchure de l'Escaut et la Corogne (incl. îles anglo-normandes).

Un vol. in $-8^{\circ}$ de 260 pages

par J. Chalon, Dr en sc. nat., prof. à l'Institut des Htes études de Bruxelles.

Envoi franco dans l'Union postale, contre mandat de 6 franis à l'auteur, $\mathrm{S}^{\mathrm{t}}$-Servais. Namur. Belgique.

\section{SMITHSONIAN INSTITUTION LIBRARIES}

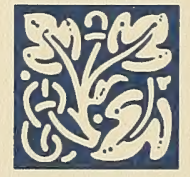

From the Library of

E. YALE DAWSON 
LIBRAFY OF

$E \times Y, D A W S O N$

\section{LISTE}

DES

ALGUES MARINES 


\section{J'offre mes remerciements à}

Monsieur le $\mathrm{D}^{\mathrm{r}}$ Yves Delage, qui m'a toujours si bien accueilli en son Laboratoire de Roscoff, mettant à ma disposition tout le matériel de pêche et de travail, et qui m'a ainsi rendu possible l'étude algologique de la région;

Mon ami le professeur Van Heurck, qui a bien voulu me communiquer le manuscrit de son Prodrome, longtemps avant l'impression (voir p. 6);

M. Malard, sous-directeur de Tatihou, qui m'a communiqué le manuscrit de ses Notes d'herborisations algologiques (voir p. 213);

MM. Heydrich et Reinbold, les éminents Algologues, qui m’ont aidé à déterminer un grand nombre d'espèces difficiles; $M$. Heydrich en outre a revu la synonymie de mes Corallinées;

Mesdemoiselles Vickers et Karsakoff, qui en 1898 m'ont vraiment initié à l'Algologie, par la collection de spécimens qu'elles ont bien voulu me donner, et par les déterminations qu'elles ont bien voulu faire de mes premières récoltes. 


\section{LISTE}

DES

\section{ALGUES MARINES}

OBSERVÉES JUSQU'A CE JOUR

ENTRE L'EMBOUCHURE DE L'ESCAUT ET LA COROGNE

(INCL. ILES ANGLO-NORMANDES)

PAR

\section{J. CHALON}

DOCTEUR EN SCIENCES NATURELLES

PROFESSEUR A L'INSTITUT DES HAUTES ÉTUDES DE BRUXELLES

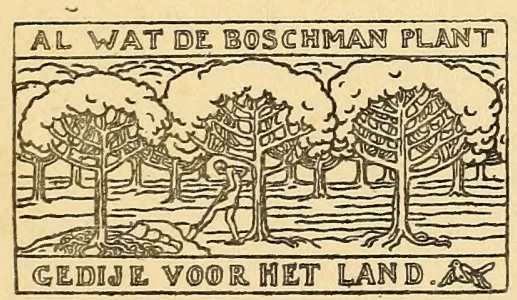

ANVERS

IMPRIMERIE J.-E. BUSCHMANN

REMPART DE LA PORTE DU RHIN

FÉVRIER 1905. 



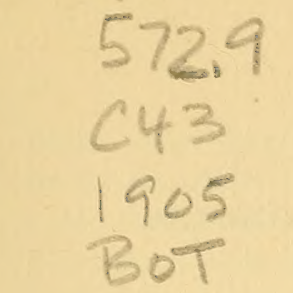

\section{SOURCES DES RENSEIGNEMENTS}

\section{LABORATOIRES MARITIMES}

Wimereux. Directeur : Giard.

Dieppe. Collège de France. Directeur : C. Houlbert.

Le Portel à 5 kilom. de Boulogne-sur-mer. Fondé par M. Bétencourt. Directeur : Hallez.

Boulogne-sur-mer. Station aquicole. Directeur : le docteur Eugène Canu.

Tatihou. Muséum d'hist. nat. de Paris. Directeur : E. Perrier.

Luc. Faculté des Sc. de Caen. Directeur : Joyeux-Laffuie.

Roseoff. La Sorbonne. Directeur : Yves Delage.

Concarneau. Directeur : Fabre Domergue.

Arcachon. Soc. scientifique d'Arcachon. Directeur : Jolyet. Avec une petite annexe à Guéthary.

Tous ces laboratoires sont attribués à la Zoologie. Un travailleur botaniste en sera-t-il exclus? Je ne le crois pas, étant donnée l'extrême courtoisie de nos voisins français, dans tous les domaines, et surtout dans le domaine scientifique. Rappelons que M. Giard, directeur de Wimereux, connu spécialement par ses travaux de zoologie, est aussi un botaniste de grande valeur.... et que j'ai été moi-même accueilli à Roscoff, et par M. De Lacaze et par M. Delage, avec une bienveillance extrême, malgré la modestie de mes recherches, qui ne dépassaient guère la simple herborisation.

\section{MES HERBORISATIONS}

Wimereux. Mai 1901.

Carantec. Août 1903.

Roscoff. Août 1898. Septembre 1899. Avril 1901. Août-septembre 1901. Août-septembre 1902. Septembre 1903.

Camaret, Toulinguet, Pen-Tir. Août 1904. 
Le Croisic. Juin 1903.

St-Jean de Luz et Biarritz. Avril 1901.

Guéthary, Biarritz. Mai-juillet 1903. Septembre 1904.

\section{ÉPAVES}

Certaines espèces arrivent habituellement à la côte en épaves; ce sont celles qui habitent les zones inférieures aux plus basses marées, ne découvrant jamais, par exemple Codium Bursa, Naccaria Wiggii, Desmarestia viridis, Bonnemaisonia asparagoides. Parfois l'ensemble des faits porte à croire que la vraie station de l'espèce est proche: tel le Sargassum flavifolium de Guéthary; certains dragages heureux confirment la prévision. D'autres fois on sait que l'Algue arrive de loin, par exemple Sargassum bacciferum et Vidalia volubilis à Ostende; on ne peut la compter comme appartenant à la Flore du pays - pas plus qu'on ne peut inscrire dans la Flore d'Islande les troncs d'arbres mexicains que le Gulf-Stream $\mathrm{y}$ amène.

La Liste note donc l'origine épave chaque fois qu'il a été possible de la constater.

\section{PROFONDEURS}

Les grandes profondeurs sont difficilement accessibles; elles contiennent probablement beaucoup d'espèces que nous ne connaissons pas encore. Le Laminaria Rodriguezii ne se développe que sous une centaine de mètres d'eau; aussi la découverte en est relativement récente, quoiqu'il soit de grande taille. A cent mètres l'eau reste calme pendant les plus violentes tempêtes, et n'envoie jamais d'épaves vers les rivages.

Les dragages se font généralement pour le service de la Zoologie, sur fonds de sable, de vase ou de gravier fin, où les Algues ne s'implantent pas. Les roches accidentées ne se prêtent pas à l'exploration. Cependant, par la drague, M. Rodriguez, autour des Baléares, a pêché des choses merveilleuses.

Mademoiselle A. Vickers à la Barbade employait des nègres, plongeurs habiles, qui arrachaient et remontaient plantes et galets chargés de végétation.

A Guéthary, M. Sauvageau a trouvé des aides précieux dans la personne des crabes Maïas, qui vivent à d'assez grandes profon- 
deurs; leur carapace épineuse accroche les Algues mieux que les meilleurs fauberts, et peut en outre servir de substratum permanent à des espèces de petite taille. Je ne sais pas si l'on a exploré à ce point de vue les Maïas qu'on prend en si grand nombre autour de Belle-Isle et dont on décharge à Quiberon de pleins bateaux.

Les piéges à homards, dits casiers, rapportent souvent de 50 mètres, plus ou moins, de fort bonnes espèces. On les surveillera soigneusement quand les pêcheurs les remontent; soit à Guéthary en juillet, août et septembre.

Les filets des pêcheurs et les piéges à homards fournissent à Roscoff Desmarestia, Arthrocladia, Bonnemaisonia etc. Quand la mer est houleuse, la moisson arrive abondante.

\section{LES HERBIERS}

Formes des Fucus de la côte belge. Herbier des échantillons authentiques de Kickx, conservé au collège des Jésuites de Namur.

Les herbiers conservés au Jardin des Plantes de Caen sont extraordinairement riches en Algues. Ils forment une série de collections séparées, non fondues en herbier général; citons les noms de Lenormand, Chauvin, Brébisson, Lamouroux, Pelvet. Grâce à la complaisance de M. le $\mathrm{D}^{\mathrm{r}}$ Lignier, doyen de la Faculté des Sciences de Caen et conservateur des collections, j'ai pu explorer l'herbier Lenormand. La section algologique seule de cet herbier comprend 28 énormes volumes; les échantillons y sont en parfait état. Le travailleur qui voudrait y faire des recherches suivies serait récompensé par de bonnes trouvailles, ignorées sans doute de Lenormand lui-même, par exemple les espèces de création récente.

Herbier du Jardin botanique de Bruxelles, renfermant les exsiccata de Westendorp, Lenormand, Le Jolis, Desmazières, Lloyd etc. fondus et réunis en un seul herbier général.

Herbier H. VAN Heunck à Anvers. La partie algologique renferme, entre autres raretés, les introuvables exsiccata de Crouan et de Holmes, plus une énorme quantité d'échantillons de J.C. Agardh, miss Griffith, Le Jolis, Lenormand etc.

Herbier algologique J. Chalon. Environ 5000 espèces et variétés, représentées par 15,000 échantillons. 
Herbier Zanardini conservé au Musée civique de Venise; 2425 numéros. Catalogue publié en 1888 par De Toni et David Lévy.

Algues de la Normandie par Chauvin, 7 fascicules de 25 espèces, publiés en 1827 et années suivantes. Les Reliquiae Chanvinianae ont été mises en vente, après la mort de l'algologue, en 1860.

Desmazières. Plantes cryptogames de France, $1^{\text {re }}$ édition, 44 fascicules de 50 espèces, 1825-1851; 2e édition, 37 fascicules de 50 espèces, 1836-1851; édit. nouvelle, 16 fascicules de 50 espèces, 1853-1860.

La $1^{\text {re }}$ édit. renferme donc 2200 numéros. Les mêmes espèces se représentent en partie dans les éditions 2 et 3, sans aucune nouveauté; ce sont Champignons, Mousses, Lichens et Algues, ces dernières en minorité. Par exemple, Desmazières ne donne que 15 Polysiphonia. Desmazières est mort en 1862.

Sirodot, professeur à la Fac. des Sc. de Rennes. Herbier de Roscoff, déposé à la bibliothèque du Laboratoire en 1876.

\section{LES LIVRES}

- Batters. Catalogue des Algues marines d'Angleterre. Journal of Botany, 1902.

- Bornet. Les Algues de Schousboe. Publié par la Soc. des Sc. nat. et math. de Cherbourg, en 1892. Ce mémoire renferme de nombreuses indications relatives au golfe de Gascogne.

- Bornet et Flahault. Algues perforantes des coquilles calcaires. Congrès de Botanique, Paris 1889.

- Notes algologiques, par BorNet ET ThURET; deux volumes in $4^{\circ}$. De la bibliothèque $\mathrm{H}$. Van Heurck.

- Chantelat. Catalogue des plantes cryptogames et phanéro. games des environs de la Teste-de-Buch. Bordeaux 1844. (Actes de la Soc. Linnéenne, tome XIII).

- M. Colmeiro. Enumeracion de las Criptogamas de España y Portugal. Revista de los Progresos de las Ciencias, Madrid 1867.

Les Algues ont paru dans la $2^{\mathrm{e}}$ partie de ce travail, tome 18 de la Revue, 95 pages. L'auteur n'indique pas si l'Algue est récoltée en épave ou fixée.

- Florule du Finistère par P. L. et H. M. Crouan. Brest 1867. La partie consacrée aux Algues comprend 68 p. et 31 planches. Cette 
Florule a été précédée de la publication de l'exsiccata (1852) et d'un liste supplémentaire (1860).

- Debrax. Algues récoltées entre le Pouliguen et le Croisic. Ass. fr. pour l'avancement des Sc. Congrès de la Rochelle, 1882.

- Io. Florule des Algues marines du Nord de la France.

Plusieurs listes ont paru à différentes époques : dans les Mém. de la Soc. Agr. et Arts de Lille en 1883; dans les Mém. de la Soc. Linnéenne du N. de la France en 1885; et enfin dans Bull. sc. de la France et de la Belgique, en 1886 (Catalogue) et en 1899 (Florule).

- De Tonr. Sylloge.

- De Wildeman. Flore des Algues de Belgique. Bruxelles, 1896.

- De Wildeman et Durand. Prodrome de la Flore de Belgique.

- Flahault. Herborisations algologiques d'automne au Croisic. Bull. de la Soc. bot. de France, 1888.

- FosLie. Norvegian Forms of Lithothamnion. Trondhjem, 1894.

- Harvey. Phycologia Britannica. 4 vol.

- Kickx. Flore eryptogamique des Flandres, 1867, 2 vol. in $8^{\circ}$.

- Iv. Variétés de Fucus vesiculosus trouvées sur le littoral belge. Bull. Acad. roy. de Belgique, 1856.

- M. Langeron. Contribution à l'étude de la Flore du Finistère. Bull. Soc. académique de Brest, tome XXIII de la $2 \mathrm{e}$ sér. Donne spécialement les Algues du Conquet.

- D. B. Lazaro e Ibiza. Datos para la Flora algologica del Norte de Espan̆a ; dans Anal. de la Soc. esp. de Hist. nat. 1889, 20 pages. L'auteur ne distingue pas entre épave et récolte sur place. Baillière.

- Le Jolis. Liste des Algues marines de Cherbourg, 1880. Paris,

Une liste supplémentaire manuscrite, de la main de Le Jolis, m'a été communiquée par $\mathrm{H}$. Van Heurck.

- Les Algues du S. W. de la France par G. Lespinasse. Dans Ann. de la Soc. Linnéenne de Bordeaux, tome XXXVI, 1882.

- R. Moniez. Algues marines observées à Wimereux, dans Bull. sc. du département du Nord et des pays voisins, 1879.

- Sauvageau. Algues Phéosporées parasites. Journal de Botanique, 1892.

- ID. Note préliminaire sur les Algues marines du golfe de Gascogne. Journal de Bot., 1897.

- ID. Générations alternantes des Cutlériacées.. Ann. des Sciences nat., 1899.

- ID. Algues récoltées sur les Araignées de mer du golfe de Gascogne. Comptes-rendus Acad. des Sc. de Paris, 1899. 
- Sauvageau. Remarques sur les Sphacélariacées. Journal de Botanique, 1901-1903.

- ID. Quelques Myrionémacées. Ann. Sc. nat., $8^{\mathrm{e}}$ sér., $5^{\mathrm{e}}$ volume. La fin de ce travail n'était pas encore parue dans le $19^{\mathrm{e}}$ vol. de cette publication...

- H. Van Heurck. Prodrome de la Flore des Algues marines des îles anglo-normandes et du Nord-Ouest de la France. Sera mis sous presse prochainement.

\section{LES MARÉES}

L'Annuaire des Marées pour les côtes de France publiẻ chaque année par l'Imprimerie nationale de Paris (1 franc) sera le guide indispensable de l'Algologue. On y prédit les heures des hautes et des basses mers pour tous les ports de France, et la hauteur de l'eau ; en outre les mêmes renseignements pour les principaux ports de l'Angleterre et de la Belgique.

Soit par exemple la grande marée du 14 janvier 1903. Nous trouvons :

\begin{tabular}{|c|c|c|c|c|c|}
\hline & $\begin{array}{l}\text { Haute mer } \\
\text { Décimètres }\end{array}$ & Heure & $\begin{array}{r}\text { Basse mer } \\
\text { Décimètres }\end{array}$ & Heure & $\begin{array}{c}\text { Différence } \\
\text { verticale } \\
\text { Décimètres }\end{array}$ \\
\hline Socoa & 44 & 16.8 & 0 & 10.7 & 44 \\
\hline Le Boucau & 29 & 16.41 & 0 & 11.10 & 29 \\
\hline Cordouan & 53 & 16.40 & 6 & 10.14 & 47 \\
\hline Ile d'Aix & 64 & 16.12 & 3 & 10.15 & 61 \\
\hline La Rochelle & 60 & 16.13 & 4 & 10.24 & 56 \\
\hline St. Nazaire & 55 & 16.29 & 1 & 10.55 & 54 \\
\hline Port-Louis & 52 & 16.22 & 3 & 10.17 & 49 \\
\hline Brest & 78 & 16.46 & 10 & 10.42 & 68 \\
\hline St. Malo & 125 & 19.2 & 8 & 13.35 & 117 \\
\hline Cherbourg & 65 & 20.49 & 5 & 15.3 & 60 \\
\hline Le Havre & 79 & 21.54 & 6 & 17.21 & 73 \\
\hline Fécamp & 82 & 23.27 & 2 & 18.11 & 80 \\
\hline Dieppe & 94 & 23.46 & 4 & 18.13 & 90 \\
\hline Boulogne & 91 & $0.3($ Le 15) & 5) 7 & 18.56 & 84 \\
\hline Calais & 70 & 0.23 & 5 & 19.9 & 65 \\
\hline Dunkerque & 59 & 0.46 & 4 & 19.20 & 55 \\
\hline
\end{tabular}

J'ai seulement traduit ici les heures de l'Annuaire dans le cadran de 24 heures - bien plus logique que la notation française.

En effet, l'Annuaire compte les heures matin de 0 à midi, et 
soir de 0 à minuit ; par exemple midi et 10 minutes se dit 0.10 soir. Lorsqu'on dépasse midi (ou 12) dans la colonne matin, il faut pour continuer le temps sauter dans la colonne voisine, celle du soir, parce que les minutes seules, et heures + minutes, inscrites en dessous de 12 dans la colonne matin désignent des heures de nuit. Un tel saut est désagréable et compliqué.

On peut s'assurer dans l'Annuaire que la plus basse mer de chaque lieu arrive en ce lieu chaque mois toujours à la même heure. Puis chaque jour la marée, haute ou basse, se présente plus tard, suivant le mouvement de la lune, de façon à retomber juste quand se présente la même phase de la lunaison suivante : un retard de 24 heures pour 28 jours.

Prenons pour fixer les idées Brest, janvier 1904; voici les heures des basses mers :

\begin{tabular}{|c|c|c|c|}
\hline Date & Heure & Date & Heure \\
\hline 3 P. L. & 10.9 & 18 & 23.11 \\
\hline 4 & 10.56 & 19 & 23.45 \\
\hline 5 & 11.44 & 20 & 24.00 \\
\hline 6 & 12.32 & 21 & 0.17 \\
\hline 7 & 13.21 & 22 & 0.49 \\
\hline 8 & 14.13 & 23 & 1.21 \\
\hline $9 \mathrm{D} . \mathrm{Q}$. & 15.8 & 24 & 2.2 \\
\hline 10 & 16.11 & $25 \mathrm{P} . \mathrm{Q}$ & 2.45 \\
\hline 11 & 17.19 & 26 & 3.38 \\
\hline 12 & 18.30 & 27 & 4.42 \\
\hline 13 & 19.36 & 28 & 5.54 \\
\hline 14 & 20.32 & 29 & 7.7 \\
\hline 15 & 21.19 & 30 & 8.9 \\
\hline 16 & 22.00 & 31 & 9.5 \\
\hline 17 N. L. & 22.37 & $1^{\mathrm{r}}$ Fév. I & L. 9.55 \\
\hline
\end{tabular}

Il est interdit en France de puiser à la mer et d'emporter même un demi seau d'eau pour la préparation des Algues. L'Administration des Douanes m'a toujours accordé l'autorisation nécessaire demandée dans un but scientifique. 


\section{CHAMP D’EXPLORATION}

Les principaux territoires explorés par notre Liste sont :

Les vastes plages sablonneuses et pauvres, entre l'embouchure de l'Escaut et le cap Gris-Nez.

Les côtes à falaises crétacées plus ou moins consistantes et roches immergées de nature calcaire, entre lo cap Gris-Nez et le Havre, jurassiques du Havre à Valognes, le long du Calvados.

Les falaises et écueils paléozoïques et cristallins depuis Cherbourg, les îles normandes, St. Malo, jusque Brest; et depuis Brest jusqu'au Croisic. Cette région est la plus riche.

De l'embouchure de la Loire jusqu'à l'Adour, les plages sablonneuses, pauvres certainement, sur lesquelles on trouve peu de renseignements.

$\mathrm{Au}$ fond du golfe de Gascogne, et sur toute la rive $\mathrm{S}$ (espagnole) de ce golfe, les rochers calcaires, (crétacés, triasiques...) connus surtout par les explorations de M. Sauvageau. La flore du golfe de Gascogne est spéciale, peut-être à cause d'un rameau du GulfStream qui en fait le tour, d'abord le long de la rive septentrionale, puis devant Biarritz, St. Jean de Luz, St. Sébastien, puis enfin le long de la côte d'Espagne.

Nous laissons hors de la Liste :

Les plages sablonneuses et vaseuses de la Hollande, qu fourniraient jusque Helgoland une maigre récolte.

Les côtes de Portugal, trop imparfaitement connues.

Les îles Britanniques, d'abord parce que leur flore algologique a été consciencieusement recensée par Batters, et qu'on ne saurait faire mieux. Ensuite parce que cette extension de domaine nous conduirait aux espèces septentrionales, et par les Hébrides et les Orkland aux Shetland et aux Feroë. Il n'y aurait plus motif de s'arrêter.

En résumé, on peut considérer la Liste comme représentant seulement les rivages français de l'Atlantique; le supplément d'espèces donné par la Belgique et par la côte $\mathrm{N}$. de l'Espagne restant insignifiant. 


\section{L'ESGAUT}

Les abords de l'Escaut ne sont pas faciles, et ses rives vaseuses, envahies par les roseaux, ne se laissent guère explorer, ni en canot du côté eau, ni à pied du côté terre. Seules les estacades et murs des quais se montrent accessibles. Pauvre localité algologique d'ailleurs.

Les environs d'Anvers sont mieux connus que le reste de l'immense estuaire; le havre de Philippine, l'ancien chenal d'Axel ont été fouillés attentivement par Kickx.

\section{LA COTE BELGE}

Les régions uniquement sablonneuses font le désespoir des Algologues ; or, depuis l'embouchure de l'Escaut jusqu'au cap Gris$\mathrm{Nez}$, où commencent les premières falaises, on ne trouve sur cette ligne droite de 150 kilomètres, que des dunes à gauche, et des plages de sable à droite, avec, dernier refuge des Algues, quelques brise-lames, maçonnerie et clayonnage, et les estacades de Blankenberghe, Ostende, Nieuport, Dunkerque. C'est tout.

L'eau est toujours sale et limoneuse. Au large, rien que des bancs de sable et de limon, ne découvrant pas. Monotonie décourageante.

Les Algues de mer assez nombreuses inscrites dans les Flores de Belgique sont, pour la plupart, des épaves, arrachées aux rochers du Nord de la France, principalement entre Boulogne et le GrisNez, et amenées par le courant du Pas-de-Calais. Les côtes anglaises ne nous envoient rien, parce qu'il n'existe pas de courants perpendiculaires à l'axe du détroit.

Je n'ai pas pu établir nettement la liste des espèces qui ont été trouvées en place sur nos estacades et brise-lames. Par exemple le Fucus vesiculosus est indigène, c'est sûr, mais le $F$. serratus? Et le $F$. platycarpus? ? Il faudrait done une série d'herborisations attentives et consciencieuses, pour fixer exactement la population végétale de nos plages.

Mais l'entreprise offrirait peu d'attraits. Celui qui a le temps et qui veut faire de fructueuses récoltes algologiques ira à Roscoffi, ou à Belle-Isle, ou à Jersey... enfin partout ailleurs qu’à Ostende.

Sur une épave, on ne peut jamais compter; elles sont rares le 
long de nos côtes, point en grandes masses ni en gros paquets, et celui qui espérerait trouver, même dans l'espace d'une année, entre Calais et l'Escaut les 160 espèces (environ) de la Flore de M. De Wildeman s'exposerait à de fâcheuses désillusions. En outre, elles nous arrivent lacérées et macérées, et sauf les types très coriaces, Himanthalia, Ascophyllum, Fucus ceranoides, Laminaria digitata, en trop mauvais état pour faire de passables échantillons d'herbier.

J'ai cependant noté, autant que possible, pour la côte belge, les Algues clairement désignées par les auteurs soit comme épaves, soit comme fixées.

\section{DE WIMEREUX AU HAVRE}

Du cap Gris-Nez à Boulogne la côte est formée surtout de falaises appartenant au jurassique (roches en bancs horizontaux et argile); parfois de dunes, par exemple entre Ambleteuse et Pointeaux-Oies.

Au pied des falaises, des plages de sable alternent avec des cailloux roulés; parfois des éboulis de rochers énormes, recouverts à marée haute - et difficiles à franchir à pied - se chargent d'Algues.

Vers le large, à marée basse, on voit s'avancer de longues pointes formées de roches plates, à peu près horizontales, un peu relevées vers le $\mathrm{S}$. seulement. Les grandes marées se retirent à un kilomètre du bord. Parfois des éboulis de rochers sont superposés à ces tables. Une abondante végétation d'Algues les recouvre. Si la mer est très basse, on aperçoit les colonies jaunâtres du Laminaria digitata à l'extrême pointe des caps émergés.

Quand on se trouve à l'embouchure de la rivière de Wimereux, on voit à gauche des rochers chaotiques et la ruine de l'ancien château-fort, dit Tour de Croy; toute cette région est abordable aux basses mers, mais quelle circulation difficile! En longeant la côte vers la droite, on franchit bientôt un éboulis de blocs énormes, La Rochette, et l'on arrive en vingt minutes au lieu dit Pointe-auxOies. A quelque hauteur au-dessus de la mer s'élève le Laboratoire zoologique et maritime de M. Giard. Du Laboratoire même on distingue fort bien Ambleteuse, et là-bas à l'horizon, le cap Gris-Nez. Par les temps clairs, une longue bande des falaises d'Angleterre se montre à l'horizon $\mathrm{W}$. 
Comme localités algologiques aux environs de Boulogne, on trouvera d'immenses dalles, plus ou moins fissurées, abordables seulement aux basses mers - et les pilotis des grandes estacades.

La boue crayeuse qui enduit et recouvre les roches sousmarines est excessivement désagréable. Parfois on y enfonce jusqu'au-dessus de la cheville; elle souille vilainement les vêtements. Les récoltes d'Algues en sont salies à ce point qu'il faut les laver à l'eau propre avant de les mettre en papier. Les flaques d'eau à marée basse restent plus ou moins laiteuses. Cette boue s'accumule par les vents $\mathrm{N}$. et $\mathrm{N}$. E. ; elle disparaît par les vents du large.

Les localités situées entre Boulogne et le Havre sont indiquées sommairement dans Debray (op. cit.); elles ont été soigneusement explorées par Chauvin, Lenormand, Lloyd, qui ont étudié surtout les côtes du Calvados; par Debray, qui a herborisé principalement autour de Wimereux.

\section{TATIHOU}

Ce nom, d'allure polynésienne, désigne une petite île, entre St. Vaast-la-Hougue et Quineville, ancien fort déclassé. Elle possède un laboratoire de Zoologie maritime, où M. Malard, en 1898, a récolté et préparé un grand nombre d'Algues.

\section{CHERBOURG}

Je n'ai pas visité Cherbourg. Je ne sais pas s'il serait possible à un étranger d'explorer complètement cette région, à cause des ouvrages militaires et des précautions prises contre l'espionnage.

Les principales localités inscrites par Le Jolis (op. cit.) sont les suivantes (je suppose que le lecteur a sous les yeux la carte maritime de Cherbourg): Au centre, plage de sable vaseux avec Zostères, sous le quai Napoléon, depuis la jetée de l'ouest du port du Commerce jusqu'au port militaire. - Rochers de Longlet. - Murs du quai Napoléon et de la jetée de l'ouest.

En face de Cherbourg, la Digue, abordable seulement en canot, avec, à chaque bout, un fort, et vers le milieu deux autres; et l'île Pt'ée, qui porte aussi des ouvrages de fortification.

A droite : plage des Bains, depuis la jetée de l'est jusqu'au fort des Flamands. - Rochers et fort des Flamands, à 4 kil. E. de la ville. 
A gauche : bois immergés de l'avant-port militaire; murs des fossés de ce port. - Rochers du Hommet sous les fortifications du port militaire.

Dans la baie Ste-Anne, rochers d'Equeurdreville et de Haineville. Rochers sous le fort de Querqueville, à 6 kil. W. de Cherbourg. Rochers de Nacqueville, à l'W. des précédents.

\section{ILES NORMANDES}

Les îles anglo-normandes, Jersey, Guernesey, Aurigny, Sark et Herm dépendent politiquement de l'Angleterre, mais elles se rattachent à la France par leur situation géographique, leur forme, leur flore, leur géologie.

Les rochers appartiennent aux terrains primitifs, ce sont des syénites, des granits, des quartzites et des conglomérats quartzeux, des porphyres, des diorites, avec des couches très tourmentées qui rappellent les côtes de Bretagne.

Je n'ai pas herborisé dans ces îles; j’ai seulement parcouru Jersey en vulgaire touriste, et fort sommairement.

Auprès des immenses plages sablonneuses de la baie St-Aubin, qui encadrent à droite et à gauche la ville de St. Hélier, nous trouvons la baie plus petite de Ste-Brelade entre deux promontoires couverts d'Algues. La côte $\mathrm{N}$. de l'île, entièrement rocheuse et très découpée, offre une longueur de 16 kilomètres à vol d'oiseau; il faudrait tripler et quadrupler cette longueur pour en suivre les contours. Cette côte est certainement très riche, et malgré les nombreux explorateurs des îles normandes, elle réserve probablement encore bien des espèces intéressantes non signalées jusqu'à ce jour.

On consultera les travaux, herbiers et listes d'herborisation de Batters, Harvey, White, Turner, Marquand, Lelièvre, Gaudion, et surtout H. Van Heurck.

Depuis plusieurs années, ce dernier passe à Jersey les mois de janvier à mai, et ses recherches personnelles ont enrichi de plus de cent espèces la florule locale. Pour les îles normandes je ne donne aucune localité détaillée; on trouvera tous les renseignements dans le Prodome cité plus haut page 6. Je note les découvertes de H. Van Heurck avec seulement la mention: Jersey, et la date, laissant au Prodome le détail des stations. 


\section{ILE BRÉHAT}

Cette île formerait un centre excellent pour les récoltes d'Algues; elle est d'un accès facile et possède plusieurs bons hôtels d'un genre simple. Du côté $\mathrm{S}$. où l'on aborde, les rochers de porphyre rouge font un contraste étonnant avec la mer bleue quand le soleil brille. Le port clos d'où le flot se retire à cháque marée offre un très vaste champ d'herborisation; je n'ai malheureusement pas eu le temps de l'explorer.

\section{GARANTEG, L'ILE CALLOT, LE GERF}

A l'extrême pointe prolongeant la rive gauche de la rivière de Morlaix, on recontre le village de Carantec. Dans la direction de St.Pol de Léon, l'Algologue ne trouvera que des régions vaseuses stériles, ou des prairies de Zostères; mais entre la plage des bains et le château du Taureau, de belles roches baignées par la haute mer, et abordables à mer basse, seront explorées avantageusement.

Plus intéressante est l'île Callot. Pendant quatre ou cinq heures, à chaque basse mer, même en morte eau, l'île devient presqu'île et de Carantec l'on y a libre accès. Çà et là le long de ses bords, des éboulis de roches chaotiques donneront des espèces nombreuses et très belles, principalement à la pointe $\mathrm{N}$. et vers l'E.

Un peu plus loin vers le nord, à 900 mètres de la chapelle de Callot, se trouve un îlot désert, le Cerf. Dans des circonstances rares, les pêcheuses de crevettes y arrivent à pied - je ne dirai pas à pied sec; et les chercheurs de Venus verrucosa n'y perdent pas leur temps. En effet, une étroite et longue bande, le Pont du Cerf, qui par les marées de 8 déc. (Brest) émerge tout juste, réunit à Callot la pointe sud du Cerf ; à peine est-elle praticable pendant une heure. Mais prenons un canot: il s'échouera quand la mer baisse et nous emportera au flot remontant. L'excursion vaut la peine : localité très riche.

\section{ROSCOFF}

Roscoff est un des centres les plus avantageux que l'Algologue puisse visiter. Les marées de vive eau (différence de la haute mer à la basse mer 8 mètres en verticale) découvrent une immense 
étendue de roches; on peut en moyenne s'avancer partout à un kilomètre du bord, parmi les régions où foisonnent les Zostères et les Algues ; nulle part le fond de la mer n'est stérile; l'̂̂le de Bas, de nombreux îlots rocheux, ainsi que les découpures de la côte, offrent dans ces conditions des surfaces d'exploration inépuisables bien abritées contre les coups de la pleine mer.

Tous les rochers des environs de Roscoff, tous les rochers de la Bretagne, sont granitiques, ou porphyriques ; le calcaire fait absolument défaut. Or la végétation des Algues est toujours plus riche et plus belle sur roches siliceuses que sur roches calciques.

Citons au plus près de Roscoff les murs du Laboratoire et les petites jetées pierreuses, ou cales, pour l'abordage des barques; l'île Verte et ses environs immédiats; tout le pays entre cette île et la pointe de Bloscon, comprenant le port, ses jetées, d'innombrables roches saillantes et petites flaques d'eau. Ces champs sont libres à marée basse.

De la nouvelle promenade créée à l'ouest de la ville, si l'on regarde l'île de Bas, on aperçoit une tour noire, le Perroch; à pied l'on y arrive, si l'on ne craint pas de se mouiller jusqu'à la ceinture pour franchir le dernier chenal qui nous en sépare; il faut choisir naturellement le moment des plus basses mers, et ne pas s'attarder sur l'île, parce que l'eau remonte vite; le mieux sera de prendre un canot. Le trajet de Roscoff au Perroch offre de nombreuses et bonnes espèces.

Directement à l'W. du Laboratoire on voit la pointe de Perkiridic; elle porte un ancien fort converti en maison de plaisance. Quand la mer est basse, il suffit de dix minutes pour y arriver; si elle est haute, on mettra une heure au moins à contourner l'estuaire. Le Perkiridic est surtout riche en épaves que le vent d'ouest y amène; on pourra s'y rendre même aux époques de morte eau, lorsque l'herborisation est peu fructueuse ailleurs. Les masses énormes de goëmon ainsi rejetées, des milliers de mètres cubes à chaque marée, sont exploitées soigneusement par les gens du pays qui viennent les enlever sur des charrettes, les étalent pour sécher et les conservent en tas jusqu'au moment de les incorporer à la terre des jardins.

L'île rocheuse de Ledanet et le rocher du Loup, blanchi à la chaux pour servir de signal maritime, continuent vers le nord le Perkiridic. On ne manquera pas de fouiller ces localités pendant les plus basses mers. Elles sont fréquentées, ainsi que les environs du 
Perroch, par les ramasseurs de Chondrus crispus, qui emplissent de cette Algue mucilagineuse leurs barques et leurs sacs.

On emploie actuellement le Chondrus pour faire des gelées ou confitures artificielles, concurremment avec l'Agar; c'est le Caragaheen (1) des anciennes pharmacopées.

A l'ouest et à l'est de ces roches (Perkiridic, Ledanet, Loup), de vastes étendues offriront dans le sable et les Zostères des flaques d'eau ne se vidant point, et contenant de belles espèces, notamment les Laminaria saccharina géantes; puis des ruisseaux coulant vivement vers le large quand la marée descend, et nourrissant les Algues spéciales qui aiment un tel milieu, Chorda filum, Chondria tenuissima, Gracilaria confervoides.

Quand la mer s'est retirée suffisamment, nous voyons émerger des eaux profondes encore, depuis le Loup jusqu'à la pointe E. de l'île Verte, les frondes des Laminaria digitata et Saccorhiza bulbosa.

Le chenal de l'île Verte au N. de cette île, montre de farouches champs de roches éboulées que couvre l'Himanthalia; il est fort difficile d'y circuler, parce que cette espèce est particulièrement gluante et glissante ; il faudra cependant examiner les parois verticales de ces roches, soulever les puissantes végétations qui les recouvrent en retombant et sous lesquelles s'abritent de délicates espèces; s'aventurer aussi loin qu'on pourra dans le chenal lui-même, ne reculer que pied à pied lorsque l'eau commence à remonter. Dans les domaines des Laminaires, on ne peut arriver qu'un ou deux jours chaque mois, selon les lunes; et chaque jour une couple d'heures seulement. Les instants sont donc précieux. Il y a même des mois où les Laminaires ne découvrent point leurs souches.

Il est facile de se rendre à l'île de Bas. Les jetées du port ne donneront probablement rien de nouveau, ni les roches au $N$. de cette île, battues par les grandes vagues du large; mais on consacrera plusieurs herborisations aux régions rocheuses et sablonneuses de la pointe orientale; là se trouvent quelques kilomètres carrés des plus curieux; on pourra même atteindre à pied Tisaoson pendant les plus basses marées. - Ainsi se nomme l'île qu'on a devant soi si, de la chapelle Ste-Barbe, on se tourne vers le nord.

(1) Orthographe de la Pharmacopée belge. Cependant Guibourt, Histoire naturelle des drogues simples, 7 e éd. Paris 1876, t. II p. 32 et le $\mathrm{D}^{\mathrm{r}}$ Flückiger, Lehrbuch der Pharmacognosie des Pflanzenreichs, Berlin 1867, écrivent Carrageen. Il y a autant d'orthographes que d'auteurs. 
A l'est de Tisaoson, pas très loin en mer, se trouve un haut fond, coquilles et graviers, appelé Astan, où l'on va draguer volon. tiers pour la Botanique - et aussi pour la Zoologie.

En partant de la chapelle Ste-Barbe, la côte se dirige droit au sud. C'est une falaise rocheuse avec, à ses pieds, d'énormes blocs de granit; bonne région pour les explorations quand l'eau s'est retirée; il y a des passages peu commodes à franchir.

$\mathrm{Au}$ sommet de la falaise, à moins d'un kilomètre de Ste-Barbe, s'élève un petit pavillon carré, peint en rouge vif. Dans la mer, juste en face de ce pavillon, à deux cents mètres environ, un écueil, le Kaïnou, ne découvre que dans les circonstances les plus favorables. Là on se promène parmi les souches des Saccorhiza; on récolte les Corallina officinalis et squamata de grande taille, le Petrocelis cruenta, des Mesogloia, des Gelidium... Ce mot Kaïnou m'a été donné par M. Marty, du Laboratoire de Zoologie; je ne l'ai pas vu sur la carte marine.

Pempoul est le port de St-Pol-de-Léon. Si de Pempoul on remonte vers l'embouchure de la petite rivière, on rencontre le Fucus ceranoides en grands exemplaires, dans le lit même du ruisseau; et sa variété naine dans la région plus élevée, que la pluie seule arrose et la mer des très hautes marées. En remontant la rivière de Plouénan, on verra graduellement se transformer puis disparaître les Fucus vesiculosus et platycarpus.

Juste en face de Pempoul, est l'île Callot et le Cerf, dont j'ai parlé. De Pempoul on arrive à Carantec en une demi heure de mer, de Roscoff en une heure, plus ou moins, selon la direction et la force du vent et des courants.

$\mathrm{Si}$ de Ste-Barbe, on se tourne vers l'E., on aperçoit à trois kilomètres et demi, en mer, la tourelle blanche de Duon; à marée basse apparait l'énorme amas de roches noires qu'elle surmonte. C'est encore un bon gisement, une excursion classique. La grotte sous-marine de Duon, accessible quand l'eau est suffisamment retirée, sera naturellement inscrite dans le programme de la journée.

Les roches de Duon sont de syénite; déjà sombres par ellesmêmes, elles prennent une teinte de charbon mat sous une couche continue d'un Lichen, le Verrucaria Maura. Trois massifs principaux et de nombreux îlots les composent; pour passer d'un massif à l'autre, il faut nécessairement recourir au canot. La tourelle s'élève au point culminant du massif principal.

Quelques unes des crevasses qui sillonnent le formidable écueil ont juste cette profondeur : chaque mer haute les remplit, chaque 
reflux les vide; et cette largeur : on peut s'y promener, et alors sur les murs, tapissés principalement de Rhodymenia palmata, on récolte une riche moisson de Floridées.

Çà et là, des cuvettes circulaires creusées dans la roche, d'un mètre environ de diamètre sur autant de profondeur et toujours remplies d'une eau très limpide, apparaissent tapissées de Corallines d'un rose vif.

Les roches au pied de la tourelle de Duon portent en grosses touffes les différentes formes de Chondrus crispus que les gens du pays viennent récolter pendant les courtes heures des basses eaux. Une chambre où l'on accède par un escalier fruste surmonte cette tourelle, que les plus fortes mers ne recouvrent jamais.

Telles sont les grandes lignes des environs de Roscoff; quant aux détails, il faut y être pour les noter. Et telle est la richesse de cette localité qu'on ne s'étonnera point si Melles Vickers et Karsakoff ont mis dix ans à l'explorer et si, après six campagnes d'un mois ou deux chacune, il me reste encore bien des espèces à y trouver et à y étudier.

\section{BREST ET LES ENVIRONS}

Les frères Crouan ont fouillé les environs de Brest, principalement l'immense rade, dans des conditions qu'il serait bien difficile de recommencer. En effet, ils ont consacré à ce travail quinze années, et ils étaient deux, combinant leurs recherches; ils habitaient Brest, pouvaient herboriser en toute saison, profiter des marées et des coups de vent; ils ont publié un exsiccata et une Florule, stimulants puissants pour l'exactitude des indications; enfin ils avaient toute facilité de parcourir les rivages situés dans le rayon des fortifications.

Représentons-nous au contraire le botaniste étranger installé pour quelques jours à Brest, quelques semaines au plus, dans une étroite chambre d'hôtel ; et d'abord l'accès du port militaire - qui est accordé sur simple présentation d'une carte d'électeur - lui est interdit.

On ne retrouve pas sur les cartes ordinaires tous les points cités par les frères Crouan. Il est regrettable que ces derniers n'aient pas ajouté à leur Florule un plan spécial et bien documenté.

Voici quelques unes de leurs stations préférées, celles qui reviennent à chaque page de la Florule et que j'ai moi-même visitées : 
Banc du Moulin-Blanc, à l'embouchure de la rivière marine de Landerneau.

Banc de St-Marc, à 3 kil. de Brest, sur le bord de cette rivière, rive droite.

Banc du Château de Brest, à l'entrée du port militaire.

Passage de Plougastel; une barque transporte les voyageurs sur la rive g. vers Plougastel-Daoulas.

Côte nord de Plougastel, bord rocheux de la rivière.

Le Minou, avec un phare à l'entrée du goulet de Brest, côte $\mathrm{N}$. On trouve la baie de Bertheaume à l'W. du Minou, et le Mingant à l'E.

Porspoder, sur le rivage de la mer libre, au N. W. de Brest. Te Melon, non loin. En dessous, Lanildut, avec la rivière Laberildut. Plus au S. encore, Corson, l'extrême pointe W. du continent français.

Le Conquet, village de pêcheurs, à 22 kil. W. de Brest. L'Algologue pourra explorer fructueusement l'arrière-port, plus ou moins vaseux, et la presqu'île de Kermorvan. Jusque St. Mathieu il trouvera quatre kilomètres de falaises, battues par les grandes vagues de l'Océan, sans abris pour les espèces délicates, mais constituant, aux grandes marées basses, de riches surfaces d'un accès facile où les gens du pays vont faucher le goëmon. Au large, dans les îles, principalement dans l'île Molène pendant la saison d'été, fument les brûloirs où les Algues préalablement séchées au soleil sont incinérées pour l'extraction du brome et de l'iode. L'usine chimique se trouve au Conquet; les cendres y sont amenées par pleins bateaux.

\section{LA PRESQU'ILE DE GROZON}

C'est un plateau rocheux s'élevant en face de Brest de l'autre côté du goulet; ses bords représentent des falaises quartzeuses escarpées, hautes de 50 à 100 mètres, très découpées, avec comme principaux caps, la Pointe des Espagnols, des Capucins, du Grand Gouin, du Toulinguet, des Pois ou Pen-Tir, de Dinan et de la Chèvre. En certains endroits, des masses de roche schisteuse tendre, alternant avec la pierre dure, ont été attaquées par la vague, et les grottes marines de Morgat et $d u$ Toulinguet, les arches naturelles de Dinan sont célèbres.

Au pied des falaises, des éboulis de blocs énormes portent une abondante flore d'Algues, mais y cheminer n'est pas commode, et 
celui qui s'y casserait la jambe en glissant sur le goëmon à marée basse, s'y trouverait en fâcheuse posture, loin de tout secours. J'ai spécialement exploré ces éboulis à l'E. de l'anse de Camaret, au pied du Grand Gouin, au S. des falaises du Toulinguet et de la Pointe des Pois.

L'anse du Camaret s'ouvre droit au $\mathrm{N}$. du village. Par des marées de 13 (à Brest) tout le port se montre à sec. Le long des quais abondent Ulva Lactuca et Enteromorpha intestinalis crispata qui recherchent les eaux azotées des égoûts; puis viennent de grands champs de Zostère. Le long des falaises à droite et de la jetée à gauche, la teinte rouille des Fucus vesiculosus et Ascophyllum nodosum domine.

L'Himanthalia à marée très basse reste relativement peu abondante, et les Saccorhiza se montrent entièrement découverts par les marées de 9.

\section{BELLE-ISLE}

On y arrive de Quiberon en une heure de mer environ. Côtes rocheuses, avec belles falaises et riches surfaces d'herborisation pendant les grandes marées basses. Cette localité n'a pas encore été explorée complètement; elle vaudrait cependant sa liste particulière, ou Florule, qui résumerait les côtes $\mathrm{S}$. de Bretagne comme Roscoff résume les côtes $\mathrm{N}$.

\section{LE MORBIHAN}

Une mer intérieure avec plus de cinquante îles habitées et un grand nombre d'îlots; une mer sans tempêtes mais avec de puissantes marées qui font au goulet quatre fois par jour de terribles courants. Les côtes intérieures du Morbihan sont encore peu connues au point de vue algologique.

\section{LE GROISIC}

Déjà avant d'arriver au Croisic, station terminus, le train longe les vastes échiquiers des marais salants, qui offrent une flore saline absolument spéciale; peu variée d'ailleurs.

La mer ouverte se trouve à un kilomètre de la gare; droit au 
sud; de nombreux rochers, déchiquetés par le flot, s'étendent à droite et à gauche de la petite plage balnéaire de Port-Lin ; riches champs d'herborisation.

Si de la gare nous nous dirigeons vers l'ouest, nous parcourons entre la ville et le port la longueur du quai. Séparé du Traict par une série de terre-pleins, ou blocs de maçonnerie, laissant entre eux les ouvertures par lesquelles entrent et sortent les barques de pêche, ce port reste sec à chaque marée basse.

Le Traict, ce sont, au-delà des terre-pleins, donc vers le nord, d'immenses bas-fonds, presque entièrement à découvert à chaque marée basse, sablonneux et vaseux. Ils se continuent vers l'E. par les marais salants et vers l'W. ils s'ouvrent dans la pleine mer.

La presqu'île en face et au N. du Croisic, reconnaissable aux vastes bâtiments réguliers qu'on y a construits, se nomme Pen-bron.

Continuons à nous avancer sur le quai principal : il se prolonge par une jetée puissante que termine un phare. A gauche de cette jetée, donc en mer libre, de grandes étendues sont occupées par des rochers que chaque marée découvre, laissant entre eux des flaques: station plus riche et mieux abritée que les falaises de Port-Lin.

M. Flahault a exploré parfaitement ces différentes localités et nous en a fait connaître la florule dans le mémoire cité plus haut.

\section{LE BASSIN D'ARGACHON}

Côtes sablonneuses, donc pauvres. Il faudra rechercher le Bostrychia scorpioides, presque aérien, parmi les Obione et les Salsolacées des vases salées; dans les pares à huîtres, la florule ordinaire des petites Chlorophycées et Myxophycées, et sur les écales d'huîtres, les Algues perforantes.

La Teste-de-Buch, port de cabotage au sud du bassin, nous est surtout connue par le catalogue de Chantelat. En raison de la nature de la côte, la plupart des Algues citées par cet explorateur ne représentent que des épaves.

\section{BIARRITZ}

Biarritz est un promontoire rocheux entre deux interminables plages de sable qui s'étendent au $\mathrm{N}$. et au $\mathrm{S}$.; roche calcaire très tourmentée et caverneuse offrant sur de grandes surfaces, et notam. ment non loin de l'îlot de la Vierge, le curieux alignement des 
Oursins au fond de sillons, avec la même régularité que si les animaux avaient été placés pour collection dans des tiroirs. Les séries parallèles sont espacées entre elles de 20 ou 30 centim. et d'une longueur indéterminée; les Oursins s'y succèdent à quelques centimètres les uns des autres, encadrés chacun par un rebord d'Algue calcaire (Lithothamnion incrustans), de sorte que les animaux, noirs, ou vert bronze, ou blanc rosé, vivent au fond d'un égal nombre de petits puits dont les bords ressemblent à des choux-fleurs roses. Le développement des Lithothamnion atteint à Biarritz une puissance considérable.

Un autre phénomène naturel intéressant, ce sont les marmites pareilles à celles de Duon, mais ici creusées dans les bancs de calcaire bien plus friables que les syénites de Duon. Elles ont ordinairement 60 cent. de diamètre, circulaires, sur 70 cent. à $1 \mathrm{~m}$. de profondeur. Des Algues calcaires blanches ou rosées en forment les parois ; une eau limpide comme le cristal les remplit. Le fond et les parois sont tapissés d'Oursins, chacun au fond de sa logette d'Algues pierreuses roses.

Il faudra surtout explorer à Biarritz les rochers aux environs de l'îlot de la Vierge ou Cucurlon, le Port des Pêcheurs, au N. de cet îlot, et surtout le Port-Vieux au S.; les grottes marines sous l'îlot, les roches entre le Port-Vieux et la villa Belza, et jusque sous la villa elle-même. Ce seul champ, quoique restreint, fournira de riches récoltes, et l'on pourra y retourner à plusieurs reprises avantageusement ; la flore varie beaucoup suivant les saisons.

Les rochers de la Chinaougue, tout près et en dehors du Port des Pêcheurs, offriront en hiver le rarissime Polysiphonia Schousboei Thur.

Le Boucaleau est un îlot rocheux très difficilement abordable, à une petite distance du Cucurlon. Je n'y ai pas été.

L'A talaya est le grand massif rocheux qui sépare le Port des Pêcheurs du Port-Vieux. La base rocheuse est abordable par mer basse; on a pratiqué dans la falaise un escalier sommaire qui permet d'y descendre... et surtout d'en remonter quand l'eau revient.

Il est assez inutile d'aller perdre son temps à la grande Plage, au N. du Port des Pêcheurs, ou sur la Plage des Basques, au S. de la villa Belza. 


\section{DE BIARRITZ A GUÉTHARY}

Tout au bout de la Plage des Basques, nous rencontrons la masse rocheuse de la Goureppe, des plus intéressantes à marée basse, et susceptible de fournir quelques herborisations bien fructueuses. La haute mer recouvre entièrement ces roches, poreuses et fort accidentées. On y arrive soit de Biarritz en une demi-heure, soit de Guéthary en une heure - si l'on marche bien.

On rencontre ensuite, isolées et dressées sur le rivage, deux roches de calcaire dur, les Roches Blanches, avec des espèces intéressantes qui ne se trouvent pas ailleurs, notamment le rare Ophidocladus simpliciuscula. On les aperçoit de loin, même de Guéthary, dressées au bord de la mer qui les assaille de toutes parts. Dans le sable près des Roches blanches j'ai cueilli des Ahnfeltia de 30 centimètres de hauteur.

Continuons à nous avancer vers Guéthary. Ce rocher qui ressemble à un colossal champignon, rongé vers la base par le choc des vagues, c'est la Roche-qui-boit. Elle s'écroulera un jour : simple question de siècles.

Un peu avant d'atteindre les bains de Guéthary, à cent mètres peut-être de ces bains, voici de vastes plaines rocheuses, plus ou moins accidentées, de pierre dure à crêtes relevées qui laissent entre elles des flaques pleines d'Algues. On peut s'avancer très loin vers le large, arriver dans la région des grandes Laminaires (Saccorhiza) et revenir porteur de riches récoltes. Je n'ai vu nulle part ailleurs en aussi grande quantité le Liagora viscida.

Au-delà de Guéthary, d'autres plateaux rocheux de même nature que les précédents, un peu plus accidentés, constituent le massif d'Arotcha. Sur la grève proche s'élève un tout petit bâtiment, Annexe de la Société scientifique d'Arcachon, qui pourrait devenir un jour laboratoire de recherches maritimes... Formons des vœux ! car la région est admirable.

Entre les crêtes rocheuses d'Arotcha et le rivage, on voit deux ou trois bassins peu profonds, où l'on a essayé autrefois la culture de l'huître. Il ne reste rien aujourd'hui de ces tentatives; le fond de ces bassins est entièrement tapissé par les Lithothamnion, et envahi par les Oursins, des milliers et des milliers d'Oursins, noirs et roses. 


\section{ST. JEAN DE LUZ, SOCOA, STE. BARBE}

En face de la ville de St. Jean de Luz s'étend une immense plage de sable - donc absolument stérile pour nous ; mais à droite et à gauche, c'est-à-dire à l'E. et à l'W. commencent les falaises.

Près de la ville elle-même on pourra explorer les bassins établis à l'embouchure de la rivière marine la Nivelle; le flot les emplit et les vide à chaque marée; des paquets de Fucus ceranoides en garnissent les murailles.

En outre, de l'autre côté du chemin de fer, les anciens parcs aux huîtres aujourd'hui abandonnés nourrissent un grand nombre d'espèces qui aiment les eaux saumâtres, la vase, les marais salés rarement noyés par la mer.

A deux kil. W. de St. Jean de Luz nous trouvons le Château fort et le phare de Socoa. Les bancs calcaires (crétacé) parallèles à la côte sont relevés, presque verticaux, et forment au fond de la mer une alternance de crêtes et de sillons, favorable au développement des Algues, mais nullement à la circulation de l'algologue. Les falaises en outre ne présentent ni rampes d'accès, ni escaliers, et il serait vraiment dangereux de se laisser surprendre par la marée au cours d'une herborisation.

D'ici nous voyons s'allonger en ligne droite ces falaises farouches, jusqu'à l'embouchure de la Bidassoa ; on distingue nettement à condition que le temps soit bien clair, le cap Figuier qui s'avance au-delà de Fontarabie sur la rive gauche de ce fleuve franco-espagnol; l'horizon se ferme par le rideau plus lointain et très estompé des Pyrénées qui forment le bord sud de la mer Cantabrique.

Du côté opposé à Socoa, à l'E. de la rade de St. Jean de Luz, nous atteignons la jetée de Ste-Barbe qui s'avance dans la mer, haute et puissante; au moyen d'échelles en fer, verticales, on peut descendre sur la roche, quand le flot est bas. De part et d'autre de cette jetée, on cueillera un grand nombre de spécimens intéressants; notamment sur les blocs du côté de la ville le nain Fucus limitaneus.

Autour de Socoa, autour de Ste-Barbe, l'eau reste toujours claire et les plantes propres. En toute saison les récoltes y sont abondantes. Après les coups de vent et le gros temps, après le mouvement particulier de l'eau que les marins appellent lames de 
fond, ou mer de fond, on trouve d'abondants paquets d'épaves sur l'un ou l'autre point de la côte selon la direction du flot.

\section{ROCHERS DE STE-ANNE, ÉCUEIL D'ANDAGORRIA}

Ils forment un groupe puissant, à l'extrémité de la falaise de Socoa. On peut aisément les atteindre de Hendaye (4 kil.).

La partie de ces roches à l'intérieur des deux tours naturelles que la mer haute entoure de toutes parts, offre une intéressante herborisation; j'y ai trouvé notamment une surface de plusieurs hectares tapissée uniquement par une forme curieuse du Fucus limitaneus Mont.

\section{LE GAP FIGUIER}

Excursion très intéressante; de Fontarabie, il y a environ une heure de marche; on atteint le sémaphore par une route commode. Ensuite il faut descendre jusqu'à la mer. Des blocs de rochers éboulés, énormes et chaotiques forment le fond à parcourir entre le cap et l'île Amuro ; ils portent çà et là des gazons épais de Fucus limitaneus réduit et d'autres gazons absolument ras de Gelidium pusillum et de Laurencia pinnatifida.

Il faut bien calculer l'heure du flux et ne pas se laisser sur prendre dans l'île. Ce serait fort ennuyeux d'y passer la nuit; les barques, je pense, n'y sauraient aborder.

Du côté N. les bancs de rochers forment des crêtes se perdant sous l'eau vers le large; il est difficile déjà de les atteindre, plus encore de les parcourir et explorer. II ne faudrait pas non plus songer à y arriver en barque, à moins que la marée ne coïncidât avec un calme plat. Quant à retourner vers Fontarabie par le pied des falaises, l'excursion prendrait beaucoup plus de temps que la durée de la basse mer.

Pendant tout le trajet, depuis le sémaphore jusque Fontarabie, on a en vue les falaises de Socoa, lisses et brillantes comme une muraille, et le groupe des rochers d'Andagorria. 


\section{LA COTE SUD DU GOLFE DE GASCOGNE}

Les principales localités de cette longue côte (près de 600 kilomètres) ont été sommairement esquissées, au point de vue algologique, par M. Sauvageau (op. cit.): San Vicente, Gijon, Rivadeo et la Corogne. D'autres points sont cités dans les listes de MM. Colmeiro et Lazaro, mais non décrits. Ces régions sont encore imparfaitement connues, elles devront être l'objet de nouvelles et patientes explorations, puisqu'on peut encore trouver du nouveau sur les côtes du Calvados, à Jersey, à Roscoff, à Brest, qui depuis plus de cinquante ans, ont servi de champ d'herborisation à tant de chercheurs ! 


\section{AMÉLIORATIONS FUTURES}

Il est possible que les recherches ultérieures des botanistes apportent à la Flore française des espèces nouvelles, même des groupes d'espèces, comme les Algues perforantes des coquilles de MM. Bornet et Flahault ou les Ectocarpus parasites de M. Sauvageau.

Espérons aussi que certains groupes (Ulva, Gelidium, Enteromorpha, formes des Fucus), seront mieux connus, et simplifiés; quoique les Rosa, les Rubus, les Hieracium... ne semblent pas devoir être débrouillés prochainement.

Il est probable qu'on trouvera de nombreuses stations nouvelles aux espèces déjà connues, et que pour les raretés, on précisera davantage ces stations; dans ce but, les cartes marines des côtes de France à l'échelle de $1 / 14.400$ sont excellentes et présentent un grand luxe de détails. (Paris, Challamel, rue Jacob 17).

Je recommande ces cartes pour toutes les herborisations ; elles sont d'un réel secours, permettent de préparer sûrement les excursions, épargnent beaucoup de temps. Quant aux noms qu'elles jortent, et que j'ai adoptés faute de mieux, ils ne coïncident pas en général avec les vrais noms locaux, bretons par exemple; il y aurait une révision à faire, mais ceci importe peu à la Botanique.

Les indications relatives à la Corogne sont nécessairement fort incomplètes. M. Sauvageau en effet n'y est pas resté une semaine entière, et il reconnaît n'avoir pas exploré la cinquantième partie des trois immenses baies du Ferrol, d'Ares-Betanzos et de la Corogne.

Evidemment, la Florule de Roscoff (attendue) par Melles A.Vickers et $\mathrm{N}$. Karsakoff donnera un grand nombre de stations intéressantes ; ces dames depuis plus de dix ans explorent la région dans d'excellentes conditions, avec, à leurs ordres, une embarcation, des marins du pays et des engins de pêche, pour fouiller tous les coins.

D'autre part, certains points des côtes françaises sont encore peu connus, ou inconnus. Que sait-on de l'île Bréhat, de l'île de Groix, de Belle-Isle, de toute l'immense étendue comprise entre la Loire et l'Adour, sablonneuse en majeure partie, mais offrant cependant des îlots rocheux? 
La révision attentive des grandis herbiers pourra encore mettre au jour un certain nombre de stations qui m’ont échappé.

Il se peut que j'aie inscrit dans la Liste certaines formes sous deux noms différents. Ce n'est pas dans le but de grossir la gerbe, mais seulement par ignorance de la synonymie. Le nombre considérable des synonymes pour une seule espèce, parfois vingt, trente et davantage, est une des difficultés de l'Algologie, bien faite pour rebuter les commençants. Schousboe, Kützing, appliquent à une même Algue successivement dix noms différents. Les anciennes Flores, avec d'anciens noms démodés et peu ou pas de synonymie, n'offrent qu'un faible secours; il faut nécessairement employer le Sylloge du professeur De Toni, où la synonymie est établie de la façon la plus complète, et où des tables minutieusement détaillées représentent un excellent outil de travail. J'ai trouvé aussi de précieux renseignements dans les monographies modernes, savamment travaillées, comme les Oscillariées de Gomont, les Nostocacées de Bornet et Flahault. Le travail d'élimination devra se faire surtout parmi les variétés; bon nombre d'entre elles devront être supprimées pour cause de double emploi. De Toni se montre très sobre de variétés.

La classification sera probablement critiquée. J'ai choisi, entre beaucoup d'autres, la plus simple, et seulement pour ne pas renvoyer - en suivant l'ordre alphabétique tout sec - aux deux bouts du volume des espèces très voisines, par exemple Apoglossum et Pteridium. L'ordre alphabétique, avec une table bien détaillée, suffisait à la rigueur pour un travail de ce genre, destiné exclusivement aux renseignements de localités.

Je reconnais que la division en Algues de mer et d'eau douce est absolument artificielle, nullement scientifique.

Cependant Harvey, Batters, Van Heurck, Debray, Hauck l'ont employée. Elle offre cette justification : la flore maritime est nettement délimitée, et ceux qui explorent les rivages salés n'ont pas besoin de s'embarrasser d'un livre comprenant en même temps les eaux douces. Ensuite, et surtout, la plupart des genres marins, et les grandes divisions presque entières des Phéophycées et des Floridées ne débordent point à l'intérieur des terres, restent exclusivement marins.

Autant que possible j'ai respecté la priorité des renseignements; les anciens herbiers Desmazières, Chauvin, Sirodot m'ont été précieux. Ainsi pour Roscoff, j'ai d'abord inscrit les stations de Sirodot, en second lieu celles des échantillons que Melles Vickers et 
Karsakoff ont bien voulu me donner, tout au début de mes herborisations dans cette localité; puis seulement ensuite mes propres récoltes, non comprises dans les deux séries précédentes.

La Liste mentionne un certain nombre d'Algues qui n'ont pas encore été signalées dans les limites géographiques de nos explorations, mais qu'il faudra rechercher attentivement avec espoir de les trouver.

Parmi ces inquirendae, les unes ont été observées sur les côtes S. de l'Angleterre, entre le cap Lizard et Douvres; les vents du $\mathrm{N}$. peuvent amener directement des épaves ou des spores jusque sur les côtes de France. D'autres ont été trouvées en outre dans des stations plus méridionales que la Corogne, en Méditerranée par exemple, et c'est un argument en faveur de leur présence sur les points intermédiaires.

Je n'ai porté dans les espèces à rechercher que les Algues microscopiques, échappant facilement à la vue, et dont la plantenourrice en outre appartient à la Liste.

Deux Lichens se rencontrent répandus sur de larges surfaces, couvrant les rochers de la zone supérieure et mouillés par les embruns et par les hautes marées :

Verrucaria maura Wahl. Enduit cellulaire très adhérent, d'un noir charbon. Donne une teinte noire à de grandes roches, par exemple Duon, dans la baie de Morlaix.

Lichina pygmea (Lightf.) Ag. Frutescent, d'un noir parfait, en touffes arrondies sur tous les rochers du littoral français. A été décrit comme Fucus (Lightf.) et même rangé parmi les Chondrus (Lam.).

On trouve dans plusieurs anciens herbiers (par ex. Algues de Normandie de Chauvin, $\mathrm{n}^{\text {os }} 102$ et 131) et considérés comme Algues, des Alcyonidium, qui sont des Bryozoaires gélatineux,

Le Cryptococcus roseus Ktz. indiqué par Crouan sur la digue de Goulven, et par Thuret à Querqueville, est une Bactérie. 


\section{STATISTIQUE}

Foslie, île de Wight . . . . . . . . . . . . . 208

Harvey, îles Britanniques . . . . . . . . . . . . 388

Debray, Nord de la France. . . . . . . . . . . . . 275

Batters, espèces et variétés. . . . . . . . . . . . 1045

Le Jolis, Cherbourg . . . . . . . . . . . . . . . 396

Crouan, Algues marines exsiccata (1852) . . . . . . . . 404

Id. Liste supplémentaire de cet exsiccata (1860). . . . 128

Id. Florule du Finistère, esp. et var., non compris Algues d'eau douce (1867) . . . . . . . . . . . . 688

Flahault, le Croisic. . . . . . . . . . . . . . . . 230

Sauvageau, fond du golfe de Gascogne . . . . . . . . 233

Id. Gijon . . . . . . . . . . 242

Id. la Corogne . . . . . . . . . . . . . 135

Van Heurck, îles Normandes . . . . . . . . . . . . 425

Sirodot, herbier de Roscoff. . . . . . . . . . . 158

La présente Liste, espèces . . . . . . . . . . . . 844

formes et variétés . . . . . . . . 377

espèces à rechercher . . . . . . . . 92 


\section{ABRÉVIATIONS}

ZS, ZM, ZI. Zones supérieure, moyenne, inférieure. EP. Epave. W. Ouest.

Herb. Herbier. J. B. Jardin botanique.

De W. De Wildeman. Len. Lenormand.

Kx. Kickx. W. Westendorp.

Born. Bornet. Thur. Thuret.

J. CH. Chalon. Sir. Sirodot.

Le Jol. Le Jolis. H. V. H. Van Heurck.

S. Sauvageau. FL. Flahault.

D. Debray. BatT. Batters.

Chauv. Chauvin. CrN. Crouan.

Laz. Lazaro. Col. Colmeiro.

Melles V. et K. Melles A. Vickers et N. Karsakoff. 


\section{ORDRE DES MYXOPHYCÉES}

\section{GHROOGOCGACÉES}

Chroococcus.

Placoma.

Glœocapsa.

Oncobyrsa.
Aphanothece.

Merismopedia.

Polyeystis.

Chroococcus turgidus Näg.

Le Croisic. Fu.

Placoma vesiculosa (Schousb.) Thur.

Jetées de Guéthary et de St. Jean de Luz. S.

Glœocapsa crepidinum Thur.

= Protococcus Thur. - Pleurococcus Rabh.

St. Malo. ThuR. - Z. S. Quai Napoléon et jetée de l'ouest à Cherbourg ; remparts N. du port militaire. Le JoL, - Le Croisic. FL.

- La Goureppe (Guéthary). S.

\section{G. gelatinosa Ktz.}

A. aeruginea. - A la base des falaises de St-Marc. Cre.

G. granosa (Berk.) Ktz.

A la base des falaises schisteuses de St-Marc. CRN.

* Oncobyrsa marina Rabh.

$=$ Hydrococcus marinus Grun.

Signalé sur la côte $\mathrm{N}$. de la Manche. Batrt. 


\section{Aphanothece Le Jolisii Thur.}

$=$ Palmella adriatica Ktz. - Palm. mediterranea Ktz.

Murs du Quai Napoléon à Cherbourg. Le JoL. - Banc de St-Marc. CRN.

\section{$\star$ A. pallida Rabh.}

Indiqué à Weymouth. Batr.

\section{Merismopedia convoluta Bréb.}

Eaux saumâtres, anse de Kerhuon. CRN.

$$
\text { M. glauca Ktz. }
$$

Prés salés, Landerneau. CRN.

\section{Polycystis pallida Thur.}

\section{= Anacystis parasitica Ktz. - Palmella Ktz.}

Z. S. sur les Cladophora, rochers de Longlet, Querqueville. LE JoL. - Sur les Rhizoclonium, rivière marine de Penfeld. CRN. Le Croisic. FL.

\section{GHAMÉSIPHONÉES}
Dermocarpa.
Radaisia.
Pleurocapsa.
Entophysalis.
Hyella.
Chamæsiphon.

\section{Dermocarpa biscayensis Sauv.}

Sur Sargassum flavifolium EP. et sur Gymnogongrus norvegicus, Biarritz, Guéthary. S.

D. Leibleiniæ Born.

= Sphonosiphon Reinsch. - Sur Leibleinia, Schizosiphon, Lyngbya, Calothrix.

Iles anglo-normandes. Jersey 1903. H. V. H. - Le Croisic. Thur. - Sur Pylaiella litoralis, San Vicente. S.

D. prasina Born.

Wimereux, La Rochette. D. - En quantités énormes, couvrant 
des mètres carrés de rocher, sur Catenella, pointe S. de l'île Callot. J. Сн. - Sur Polysiphonia fastigiata, Roscoff. J. Сн. - Iles anglo-normandes. Jersey 1902. H. V. H. - Sur Catenella Opuntia, dans le port du Croisic. FL. - Biarritz, Guéthary, St. Jean de Luz. S.

\section{Dermocarpa Schousboei Born.}

Sur les gaînes du Lyngbya majuscula. - Biarritz, Guéthary, St. Jean de Luz. S.
D. strangulata Sauv.

Sur Sargasse, EP. à Guéthary. S.

\section{D. violacea Crn.}

Rade de Brest, dragué. CRN. - Sur Chaetomorpha, salines du Croisic. FL. - Biarritz, Guéthary, St. Jean de Luz. S.

\section{Radaisia Gomontiana Sauv.}

Sur Fucus platycarpus et vesiculosus, Biarritz, Guéthary, St. Jean de Luz. S.

\section{Pleurocapsa amethystea Rosenv.}

Iles anglo-normandes. H. V. H.

$$
\text { P. fuliginosa Hauck. }
$$

Iles anglo-normandes. Jersey 1902. H. V. H.

\section{Entophysalis granulosa Ktz.}

= Myrionema crustaceum J. Ag.

Sur une jetée en pierre à Blankenberghe. De Block. Wimereux, la Rochette, avec Verrucaria maura. De Block. Iles anglo-normandes. Jersey 1902. H. V. H.

\section{E. vesiculosa Thur.}

Biarritz. Lespinasse.

\section{Hyella cæspitosa Born. et Fl.}

Dans test des vieilles coquilles de Purpura lapillus, dans coquilles vivantes de Lasaea rubra, parmi les Balanes, etc. 
Griz-Nez, Wimereux, cap Antifer. GIARD. - Iles anglo-normandes. Jersey 1903. H. V. H. - Sur carapace de Crabe, Roscoff. J. Cr. Le Croisic. Fu. - San Vicente, Rivadeo. S.

$\star$ A. nitida Batt. $(=H$. voluticola Chodat). - Indiqué à Plymouth. Batт.

* Chamæsiphon marinus Wille.

Indiqué sur la côte $\mathrm{S}$. de l'Angleterre. Batт.

\section{LYNGBYÉES}

Spirulina.

Oscillatoria.

Phormidium.

Arthrospira.
Lyngbya.

Symploca.

Plectonema.

\section{Spirulina labyrinthiformis Gom.}

Port en Bessin. D. - Cherbourg. Thur.

S. major Ktz.

$=$ Sp. tenuissima et $S p . p s e u d o$-tenuissima Crn. $-S p . p s e u d o$. tenuissima Batt.

Embouchure de la rivière de Wimereux. D. - Carteret. Herb. ZAN. - Ile de Bas. FL. - Eau saumâtre, port de Brest. CRN. - Le Croisic. Gom. - Étang derrière la plage del Arbeyal, à Gijon. S.

S. Meneghiniana Zan.

= Oscillaria Menegh. - Sp. Zanardinii Hauck. - Sp. oscillarioides Bulnh.

Carteret. Lebel, in Herb. Thuret.

\section{S. Nordstedtii Gom.}

$=$ Sp. tenuissima Nordst.

Le Croisic. Gon.

S. rosea Crn.

Rade de Brest, à $20 \mathrm{~m}$. de profondeur. Crn. - Biarritz. Thur. 


\section{Spirulina subsalsa Oerst.}

$=$ Oscillaria oceanica Crn. - Sp. oceanica Crn. Rabh. - Sp. Thuretii Crn. Le Jol.

Eau saumâtre, ancien chenal d'Axel. Kx. - Wimereux, Croy ; plage et port de Fécamp. D. - Z. M. Longlet, Hommet, quai Napoléon. Le JoL. - Cherbourg. Thur. - Iles anglo-normandes. Jersey, sur Patelles, 1903. H. V. H. - Sur Cladophora rupestris, Duon, Roscoff. J. Сн. - Prés salés de Landerneau, port de Brest; Kervallon, Z.S.; embouchure de la rivière de Penfeld, passage de Plougastel. CrN. - Arcachon. Lespinasse. - San Vicente de la Barquera. Laz.

\section{S. subtilissima Ktz.}

Gris.Nez; Wimereux, Croy. D.

Gomont l'indique seulement dans les eaux thermales.

$\star$ S. versicolor Cohn.

A. laxa Gom. - Indiquée sur la côte N. de la Manche. Batt.

\section{Oscillatoria amphibia Ag.}

$=0$. infectoria Tassi. - O tenerrima Ktz.

La retenue de Fécamp. D.

Gomont adopte constamment le mot Oscillatoria (non Oscillaria).

\section{O. Bonnemaisonii Crn.}

$=0$. intermedia Crn. - O. colubrina Thur. in Le Jol.

Wimereux, la Rochette; Fécamp, dans le port et la retenue. D. - Cherbourg. Thur. -- Z. M. Longlet, quai Napoléon. Le JoL. Z. M. passage de Plougastel, Kervallon, rivière marine de Penfeld. CRN.

\section{O. brevis Ktz.}

A. neapolitana Gom. (= 0. neapolitana $\mathrm{Ktz} .-0$. subuliformis Le Jol, non Ktz.). - Wimereux, embouchure de la rivière. D. Z. M. quai Napoléon, Hommet, fossés des fortifications de Cherbourg. Le Jol. - Le Croisic. Thur. Gom.

\section{O. chalybea Mertens.}

Salines de Pornichet (Croisic). FL. 


\section{Oscillatoria Corallinae Gom.}

= O. capucina Crn. Holm. - O. Alcyonii Crn. - Lyngbya gracitis Batt.

Z. S. Gris-Nez, Fécamp, Arromanches, Grandcamp. D. - Iles anglo-normandes. H. V. H. - Banc du Moulin et profondeur de la rade de Brest; sur Alcyon Orange-de-Mer, banc de St. Marc. CRn. Biarritz, Guéthary, St. Jean de Luz, la Corogne. S.

\section{O. lætevirens Crn.}

Z. S. retenue de Fécamp. D. - Benouville, Bruneval. Bernard. - Bord des rivières marines, Kervallon. CRN. - Le Croisic. Gom.

Souvent mêlée à Catenella Opuntia.

\section{Lloydiana Gom.}

Pour la synonymie (O. subuliformis Thw.) voir Gomont, Bull. Soc. Bot. de France, 24 février 1899.

Z. S. Camfrout. CrN. - Salines du Morbihan. Lloyd.

O. majuscula Lyngb.

Z. M. côte N. de Plougastel, près du passage. CRN.

Eau douce? Vosges!

O. margaritifera Ktz.

$=0$. Bonnemaisonii Reinb. non Crn. -0 . insignis Thw. in Harv.

Z. S. Courseulles. BrÉB. - Le Croisic. Thur.

O. nigro-viridis Thur.

$=0$. insidiosa Crn. - Oscillaria limosa chalybea Crn. Le Jol.

St. Valéry-sur-Somme; Cherbourg. ThUR. LE JoL. - Z. M. Kervallon, quai de la Mâture etc. CRN.

\section{O. Okeni Ag.}

= Oscillaria chalybea luticola Menegh.

Gomont l'indique en eau thermale près de Liège (Chaudfon. taine ?) - Retenue de Fécamp. D.

\section{O. Pharaonis Bréb.}

= Phormidium tinctorium Ktz.

Dans la rivière de Wimereux, mais en amont du viaduc $d u$ 
chemin de fer, endroit que les marées n'atteignent guère. DE BLock. - Rivière d'Orne près de Falaise. BRÉB.

Oscillatoria rosea (Batt.) Crn.

Dragué à 20 m., Camaret. CRN.

\section{O. subuliformis $\mathrm{Ktz}$.}

Z. S. Bruneval. Bernard. - Cherbourg. Thur. - La Corogne. S.

O. subviolacea Crn.

Sur Codium adhaerens, banc de St. Marc. CRN.

O. tenuis Ag.

$=0$. natans, 0 . tergestina, 0 . viridis $\mathrm{Ktz}$.

Z. S. Gris-Nez, Fécamp, Arromanches, Grandcamp. D. - Falaise, Vire. Herb. ZaN. - Fossés de la région maritime. CRN.

Donnée par Crn. comme d'eau douce.

A. viridis. Rabh. ou Ag. - Le Croisic. FL.

Gomont indique cette variété en eau douce.

\section{O. zostericola Crn.}

Banc de Saint-Marc. CrN.

D'après Gomont, espèce à rechercher.

\section{Phormidium autumnale Gom.}

$=P h$. antliarum Gom. in Holm. et Batt. - Chthonoblastus Vaucherii West. et Wallays. - Ph. Lyngbyaceum Le Jol. - Oscillaria vulgaris et Osc. vulg. publica Crn.

Valognes (Manche). Herb. Zan. - Anse de Deolen (le Minou). CRN.

\section{Ph. corium Gom.}

$=$ Oscillaria amorpha (Berk.) Crn. Florule du Finistère p. 114. CRN.

Valognes. Herb. Zan. - Z. S. Penfeld et St.-Jean-Plougastel.

D'après Gomont, espèce d'eau douce, mais elle figure dans la liste des Algues marines de Batters. 


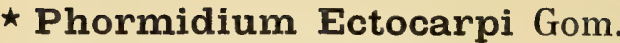

$=P h$. persicinum Batt. non Gom.

Indiqué sur la côte $\mathrm{S}$. d'Angleterre. BATT.

Ph. favosum Gom.

$=$ Oscillaria Bory. - Oscillaria antliaria Hilse. - Ph. rupestre Desmaz.

Wimereux, embouchure de la rivière, la Rochette et Croy; Z. S. Gris-Nez, Audresselles, St. Valéry-sur-Somme, la retenue à Fécamp. D. - Antifer, Bruneval. Bernard. - Port-en-Bessin. D. Cherbourg, Z. S. quai Napoléon, fossés des fortifications. Le JoL. Murs des quais, à la Mâture (donnée comme d'eau douce). CRn.

Ph. fragile Gom.

= Anabaena Menegh. - Ph. moniliforme Gom.

Sur les Balanes, Biarritz, Guéthary, St. Jean de Luz. S.

Ph. papyraceum Gom.

= Lyngbya litorea Hauck. - Oscillatoria Ag.

Caen, Z. S. à peine mouillé par le flot de la mer. Len.

$\mathrm{Ph}$. persicinum Gom.

$=$ Lyngbya Rke.

Sur Alcyonidium gelatinosum, Wimereux. D. - Sur éponge calcaire à la base d'un Cystoseira, Perkiridic (Roscoff). J. Сн.

Ph. subfuscum Ktz.

$=P h$. corium Ktz. - Oscillaria limosa subfusca Ktz. - Ph. fonticola Rabh.

Wimereux, embouchure de la rivière. D.

Ph. submembranaceum Gom.

= Oscillaria Ardiss.

Wimereux, Croy, la Rochette. D.

\section{Arthrospira miniata Gom.}

$=$ Spirulina Hauck.

Biarritz. THUR. 
Lyngloya æruginosa Ag.

A. ferruginea Ktz. (=L. ferruginea Ag.). - Caen. Chauv.

D'après Rabenhorst (Flora Europaea Algarum, vol. II, p. 138), cette forme vit en eau de mer, ou saumâtre.

B. versicolor. - Caen. Chauv.

Forme à rechercher, d'après Gomont.

\section{L. æstuarii Liebman.}

$=L$. semiplena, Oscillatoria majuscula, Calothrix semiplena Crn. - L. aeruginosa Le Jol.

Sur Moules à Blankenberghe. Kx. - Wimereux, Croy. D. Près du fort des Neiges (au Havre). Dupray. - Cherbourg, Z. M. Hommet. ThUR. - Iles anglo-normandes. Jersey 1904. H. V. H. Z. S. palue maritime de Goulven; Z. M. baie d'Audierne, St. Marc. CRn. - Vannes. Lloyd. - Le Croisic. FL. - La Rochelle. Montagne.

\section{Agardhii Gom.}

= Calothrix Crn. - Lyngbya livida Hauck.

Banc de St. Marc. CrN.

\section{L. baculum Gom.}

Sur Floridées, Biarritz. Thur.

\section{L. confervoides C. Ag.}

$=$ L. luteo-fusca sub-viridis Le Jol. - Calothrix obscura, L. obscura, L. dalmatica Crn.

Gris-Nez. D. - Sous le fort de Longlet. LE JoL. - Z. S. quai du port Napoléon III; sous le château de Brest, Trégana, banc de St. Marc. CRN. - Cap Torres à Gijon; Villavieja à Rivadeo, sur Fucus. S.

\section{L. lutea Gom.}

$=L$. semiplena Hauck et Richt. - L. Juliana Crn. Desmaz. - L. microscopica Crn.

Gris-Nez. D. - Arromanches. Herb. Zan. - Z. M. anse de Kerhuon; Z. S. bords de la rivière marine de Penfeld. CRN. Biarritz, sur rochers maritimes où suinte l'eau douce. Bors. Biarritz, Guéthary, St. Jean de Luz. S. 


\section{Lyngbya majuscula Harv.} Ag.

$=L$. ferruginea Desmaz. - L. luteo-fusca Crn. - L. crispa

EP. chenal de Nieuport. Kx. - Z. S. Cherbourg. LE JoL. Iles anglo-normandes. H. V. H. - Roscoff. Sir. - Entre le Laboratoire et l'île Verte. J. Сн. - Bane de St. Mare, Z. M. Crn. Le Croisic. Fu. - Port du Croisic. D. - La Teste-de-Buch. Chantelat. - Z. I. Sur le sable, Biarritz, Guéthary, St. Jean de Luz, San Vicente, Gijon, la Corogne. S.

\section{Meneghiniana Gom.}

$=$ Leibleinia Ktz.

San Vicente, cap Oriambre, sur un Polysiphonia; Rivadeo, Villasellan, sur Castagnea chordariiformis. S.

\section{Phormidium Ktz.}

Rivière marine de Penfeld. CRN.

D'après Gomont, espèce à rechercher. Sans échantillon authentique, il est impossible de la placer dans aucun genre précis. La description et la figure ne suffisent pas à montrer s'il s'agit d'un Lyngbya, d'un Symploca ou d'autre chose.

\section{* L. Rivulariarum Gom.}

Dans les gaînes gélatineuses de Rivularia Biasolettiana et de Microcoleus chthonoplastes. Indiqué sur la côte N. de la Manche. BATT.

\section{L. semiplena J. Ag.}

$=L$. sordida Ktz. Le Jol. - L. congesta Crn. - L. confervoides Batt.

Gris-Nez, Wimereux. D. - Z. S. Nacqueville; au-dessus du fort de Querqueville. Le JoL. - Cherbourg. Len. - Iles anglo-normandes. H. V. H. - Kervallon, Z. M. et Z. S. Bane de St. Mare, Z. M. CRN. Le Croisic. Fu. - Rivadeo. S.

A. viridis Crn. - Z. S. St-Marc. CRN.

\section{L. sordida Gom.}

$=$ Leibleinia violacea Born. - L. violacea Hauck. - L. Griffithsiae Crn. - L. polychroa Thur.

Sur Griffithsia secundiflora; banc du Château de Brest. CRN. 
- Biarritz. ThUR. - Biarritz, sur différentes Algues. Born. Guethary. Lespinasse. - Gijon. S.

\section{Lyngbya stragulum Ktz.} CRN.

Hommet, baie Ste-Anne. LE JoL. - Rivière marine de Penfeld. D'après Gomont, espèce à rechercher.

$$
\text { L. Mucor (Ag.) Crn. }
$$

Banc de St. Mare. Crn.

\section{Symploca atlantica Gom.}

Le Croisic. Bonn. - Biarritz. Bons. - Gijon. S. BATT.

* A. purpurea Batt. - Signalée sur les côtes du Devonshire.

\section{S. hydnoides Ktz.}

= Calothrix Crn. - S. Harveyi Le Jol. - Schyzosiphon Crn. - S. Catenellae Hauck. - Calothrix semiplena Harv.

Côtes de la Manche. Len. - Sur Rytiphloea pinastroides, Querqueville. LE JoL. - Iles anglo-normandes. H. V. H. - BelleIsle en mer. Lloyd. - Le Croisic. Thur. - Biarritz, Guéthary. Thur.

S. muscorum Gom.

= Phormidium spadiceum, Ph. smaragdinum et Lyngbya graveo'ens Crn. - Lyngbya Phormidium Hilse.

Murs des fossés du port militaire. LE JoL. - Z. M. port Napoléon III, rivière marine de Penfeld. CRN.

\section{* Plectonema norvegicum Gom.}

Indiqué à Swanage. Batt.

\section{$\star$ Pl. Nostocorum Born.}

Indiqué sur la côte S. d'Angleterre. Batr. Dans les gaînes gélatineuses de Rivularia bullata, Schizothrix vaginata et Dichothrix gypsophila.

Espèce d'eau douce (Bornet). 
Plectonema terebrans Born. et Fl.

Dans coquilles mortes de Vola maxima, Roscoff. J. Сн. - Dans vieilles coquilles, le Croisic. Bors. - Iles anglo-normandes. H. V. H.

\section{VAGINARIÉES}

Microcoleus.

Hydrocoleum.
Schizothrix.

Sirocoleum.

Microcoleus acutirostris Gom.

= Oscillaria microcoleiformis $\mathrm{Crn}$.

Côte N. de Plougastel. CrN.

\section{M. chthonoplastes Thur.}

$=$ Mic. anguiformis Crn. Harv. - Mic. salinus Crn.

Eau saumâtre, Wimereux. D. - Roscoff. Sir. - Baie d'Audierne, Camfrout, Kerhuon, Z. S. Sur le sable maritime, Argenton, baie du Moulin-Blanc. CRN. - Le Croisic. FL.

Forme le sol habituel des marais salants.

\section{M. tenerrimus Gom.}

$=M$. salinus Hansg.

Sur Rhodochorton Rothii, cap Antifer. D. - Le Croisic. Gom.

Hydrocoleum glutinosum Gom.

= Oscillaria percursa marina Ktz. Le Jol. - Oscillaria flavo-fusca Crn.

Z. S. Querqueville, sous le fort. Thur. - Z. M. banc de St. Marc. CRN. - Z. S. Biarritz, Guéthary, St. Jean de Luz. S. - Sur Lithothamnion incrustans, la Corogne. S.

\section{H. Lyngbyaceum $\mathrm{Ktz}$.}

$=$ Microcoleus Thur. Hauck. - Oscillaria Iitoralis Crn. Rabh. Desmaz. - Phormidium Kutzingianum Le Jol.

Griz-Nez; Wimereux, Croy ; Port-en-Bessin, Grandcamp, D. Remparts du port militaire de Cherbourg. Le JoL. - Z. M. baie du 
Moulin-Blanc, Penfeld, banc de St. Marc. CRN. - Rivadeo, mélangé à Lyngbya Meneghiniana. S.

A. rupestre Ktz. (= Microcoleus nigrescens Thur.) - Côtes de la France du Nord. Le Jol. in Herb. Thur.

\section{Schizothrix Gresswellii Harv.}

= Calothrix Harv. - Inactis Thur.

Biarritz. Lespinasse.

$\star$ S. lardacea Gom.

$=$ Hypheothrix coriacea Rabh.

Indiqué sur la côte $\mathrm{N}$. de la Manche. BatT.

D'après Gomont, espèce d'eau douce. Figure dans la liste des Algues marines de Batters.

S. vaginata Gom.

= Inactis Näg. - Inactis scopulorum Thur.

Eaux saumâtres. Côtes W. de France. Thur.

\section{Sirocoleum Kurzii Gom.}

= Microcoleus penicillatus Thur. - Micr. Kurzii Gom.

Le Croisic. Born. - Biarritz. Herb. Thur.

\section{RIVULARIÉES}

Amphithrix.

Calothrix.

Dichothrix.

Isactis.

Rivularia.
Brachytrichia.

Dasyactis.

Gloeotrichia.

Physactis.

Schizosiphon.

Amphithrix violacea Born. et Thur.

= Hypheothrix Ktz.

Calvados. BRÉB. - Brest. CRN.

Calothrix æruginea (Ktz.) Thur.

$=$ Leibleinia (Ktz.) Le Jol. - Leibl. Nemalionis Crn.

Gris-Nez, Grandchamp. D. - St. Vaast. HerB. ZaN. - Z. S. 
Hommet, Querqueville. Thur. - Roscoff. Dét. Heydrich. J. CH. Passage de Plougastel, ile d'Ouessant. CrN. - Le Croisic. FL.

\section{Calothrix confervicola Ag.}

= Oscillatoria Ag. - Leibleinia chalybea et L. amethystea Ktz. Le Jol. - C. confervicola zostericola Crn.

Z. M. Gris-Nez; Wimereux, la Rochette ; Port-en-Bessin, Grandcamp. D. - Z. M. Cherbourg; Hommet, plage des bains. LE Jol. Iles anglo-normandes. H. V. H. - Roscoff. Sir. - Banc de St. Marc, le Minou. CRN. - Le Croisic. FL. - Gijon. S. - San Vicente de la Barquera. Laz.

\section{Contarenii Born. et Fl.}

= Rivularia Rabh. - Mastichonema Ktz.

St. Malo. Bonn. - Le Croisic. FL. - Sur Patelles. Biarritz, Guéthary, St. Jean de Luz. S.

\section{G. crustacea Thur.}

= Schizosiphon lasiopus Ktz. Le Jol. - Schizosiphon fasciculatus Le Jol.

Wimereux, Pointe-aux-Oies. D. - Z. S. Nacqueville, Landemer. LE Jol. - Le Croisic. FL. - Sur Patelles, et enduit sur roches. Biarritz, Guéthary, St. Jean de Luz. S. - San Vicente, Rivadeo. S.

\section{C. fasciculata Ag.}

= Schizosiphon fasciculatus Ktz. Rabh. - C. scopulorum Aresch.

Z. S. Equeurdreville. Le JoL. - Z. S. baie du Moulin-Blanc. CrN.

C. fusco-violacea Crn.

Dragué rade de Brest. CRN. - Z. I. ne découvrant pas, le Croisic. FL.

\section{G. parasitica Thur.}

= Rivularia Chauv. - Schizosiphon Le Jol. Crn.

Querqueville. LE JoL. - Iles anglo-normandes. H. V. H. - Dans Nemalion lubricum, batterie du Diable, le Minou. CRs. - Dans Nemalion multifidum, le Croisic. FL. - Biarritz, Guéthary, St. Jean de Luz. S. 


\section{Calothrix parietina Thur.}

= Scytonema salinum Ktz. - Mastichonema caespitosum Ktz.

Eaux saumâtres, Wimereux, base des falaises; Hâble d'Ault près de Cayeux-sur-Mer. D. - Palues maritimes de Goulven. CRN. - Marais salants, le Croisic. Fu.

\section{C. pulvinata Ag.}

= Schizosiphon hydnoides, S. fasciculatus, S. pannosus Crn. - C. pannosa Crn. Harv. - Schizosiphon Lenormandii Le Jol. Crn. - C. hydnoides Harv.

Z. S. sur pilotis, Ostende. W, - La Panne. DE W. - Z. S. baie Ste-Anne, Querqueville. LE JoL. - Iles anglo-normandes. H. V. H. - Roscoff. Sir. - Anse de Kerhuon, le Conquet. Z. S. Bords des rivières marines. CRN. - Le Croisic. FL.

C. rubra Born. et Fl.

- Schizothrix Crn.

Brest. CRN. - Le Croisic. FL.

\section{C. scopulorum Ag.}

= Amphithrix crustacea Crn. - Schizosiphon Sowerbyanus, S. mucosus, S. juncicola Crn. - Schizosiphon scopulorum Le Jol. Crn.

Sur pilotis et murs du port d'Ostende. FL. - Z. S. Wimereux. D. - Z. S. talus du fort des Flamands; Nacqueville. LE JoL. Iles anglo-normandes. H. V.H. - Banc de St. Marc; flaques des prés salés, Landerneau ; rivière marine de Penfeld; sous le Château de Brest, le Polygone. CRN. - Le Croisic. FL. - Biarritz, Guéthary, St. Jean de Luz. S.

\section{G. trochicola Crn.}

Dragué rade de Brest. CRN.

\section{Dichothrix gypsophila Born. et Fl.}

= Schizosiphon Ktz. - Schizosiphon rufescens Rabh.

Rochers calcaires arrosés par la mer. Côtes de France. BRÉB. in HeRB. Thur.

Espèce d'eau douce. 


\section{Isactis plana Thur.} purea Ktz.

= Rivularia Harv. - Dasyactis Ktz. - Physactis atropur-

Carteret. Herb. ZAN. - Z. I. sur Ulva Lactuca, baie Ste-Anne, Querqueville, Flamands. Le Jol. - Baie de Ste-Anne, banc de St-Marc, Laninon. CrN. - Roscoff. FL. - Le Croisic. Fl. Arcachon. Lespinasse. - Sur Fucus et Patelles, Biarritz, Guéthary, St. Jean de Luz. S. -- San Vicente, Gijon, Rivadeo, la Corogne. S.

A. zonata Thur. (Esp. distincte? Thur.) - Areachon. LesPINASse.

D'après Thuret, le Dasyactis salina Ktz. est une espèce très différente de l'Isactis plana Thur., quoique Crouan le donne comme synonyme (Florule p. 116). Voir dans Rabenhorst p. 212 la distribution géographique du D. salina Ktz.

\section{Schizosiphon trochicola Crn.}

= Isactis plana, plantes jeunes.

Dragué sur Trochus magus, rade de Brest. CRN.

Rivularia atra Roth.

$=R$. confinis, $R$. confluens, $R$. Lenormandiana Crn. Euactis West. et Wallays. Ktz. - Euactis Lenormandiana, var. Balani; Dasyactis salina Le Jol.

Sur pierres des jetées et des digues, Blankenberghe et Ostende. W. - Gris-Nez; Wimereux, la Rochette et Croy. D. - Granville, St. Vaast. Herb. Zan. - Sur les Balanes, Cherbourg. Le Jol. - Iles anglo-normandes. H. V. H. - Sur galets, Roscoff. au S. de Ste-Barbe ; sur coquille de Vola maxima. J. СН. - Z. S. St. Marc, Trégana, Laninon; sur Fucus balticus, rivière marine de Penfeld. CRN. Le Croisic. Fu. D. - Arcachon. Lespinasse. - Ile des Oiseaux (Teste-de-Buch). Chantelat. - Port-Vieux de Biarritz et jetée de St. Jean de Luz; Gijon, Rivadeo, la Corogne. S.

A. parasitica Thur. - Arcachon. Lespinasse.

R. australis Harv.

Arcachon. Lespinasse.

R. Biasolettiana Menegh.

= Schizosiphon Warrenii Casp. - Schizosiphon salinus Ktz. - Dasyactis salina Ktz.

Pilotis du port d'Ostende. Kx. - Wimereux, Pointe-aux-Oies ; Hâble d'Ault près de Cayeux sur mer. D. - Z. M. Querqueville, 
Flamands. Le JoL. - Mles anglo-normandes. H. V. H. - Falaises du bourg de Batz (Croisic). FL. - Le Ferrol. CoL. - Falaise de la pointe St. Martin à Biarritz. S. Lespinasse.

$\star$ A. confluens Farlow.

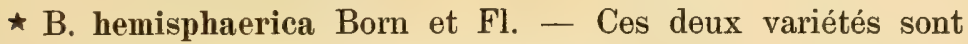
indiquées sur la côte W. de la Manche. Batт.

\section{Rivularia bullata Berk.}

$=R$. nitida Crn. Desmaz. - Physactis pilifera Ktz. Le Jol. - Physactis nitida West.

Z. M. sur pierres des jetées et Balanes à Ostende. CL. Dumont. - Nieuport. Hers. Rouzée. - Iles anglo-normandes. Jersey 1903. H. V. H. - Roscoff, sur roches à Perkiridic. J. CH. - Le Croisic. FL. - Plage du Croisic. D. - Niveau des Balanes, Biarritz, Guéthary, St. Jean de Luz, San Vicente, Gijon, Rivadeo, la Corogne. S.

R. nitida Ag.

$=R$. plicata Crn. Carm. - Physactis plicata West. et Wallays.

Z.S. sur le limon du port d'Ostende. Kx. et CL. Dumont. Z. M. Hommet, Querqueville, Nacqueville. Le JoL. - Iles anglonormandes. H. V. H. - Z. M. bords des rivières marines, Ste-Anne, Tregana, Argenton. CRN. - Dans suintements d'eau douce, bourg de Batz (Croisic). Fu. - Arcachon. Lespinasse. - San Vicente de la Barquera. Laz.

A. gracilis Crn: - Sur Palmella oceanica, rivière marine de Penfeld. CRN.

\section{R. laxa Thur.}

Arcachon. Lespinasse.

* R. mesenterica Thur.

$=R$. polyotis Holm. et Batt. non Born. et Fl. - R. bullata J. Ag. - Physactis bullata Rabh. - R. polyotis (J. Ag.) Hauck. $R$. nitida Hauck.

Trouvée à Torquay. BATT. - Côtes de France (d'après le texte, cette indication se rapporte aux côtes méditerranéennes). BonN، et FL.

R. polyotis Born et Fl.

$=R$. hospita Thur. - Physactis fucescens Ktz. - Zonotrichia hospita Rabh. - Euactis hospita Ktz. 
Quais du port du Croisic. Fr. Thur. - Biarritz. Schousboe. Rochers vaseux de la Chinaougue (Biarritz). Thur.

Brachytrichia Balani Born et Fl.

= Rivularia Lloydii Crn. - Rivularia Lloyd. - Physactis Lloydii Crn. - Hormactis Thur.

Wimereux. De Block. - Sur les Balanes, cap Antifer. D. Cherbourg. BonN et Thur. - Iles anglo-normandes. Jersey 1903, sur Codium tomentosum. H. V. H. - St. Malo. Thur. - Batterie du Diable, le Minou. CRn. - Le Croisic. FL. Thur. - Arcachon. BorN et Thur.

Dasyactis hospita (Ktz.) Crn.

= Amphithrix Ktz.

Z. M. St. Marc. CRN.

\section{Glœotrichia punctulata Thur.}

Cherbourg, en eau saumâtre. THur.

Physactis gracilis Crn.

Sur Palmella oceanica, rivière marine de Penfeld. CrN.

\section{SIROSIPHONÉES}

Mastigocoleus.

Mastigocoleus testarum Lagerh.

Iles anglo-normandes. Jersey 1903. H. V. H. - Dans coquille morte de Vola maxima, Roscoff. J. Ch. - Brest. Le Dantec. Le Croisic, dans vieilles coquilles d'Unio, et aussi dans pierres calcaires. FL. - San Vicente. S.

\section{SGYTONÉMÉES}

Microchæte.

Hassallia.

Microchæte grisea Thur.

Grandcamp. D. - Brest. CRN. - Sur vieilles coquilles, le Croisic. FL. Born et Thur. - Rivadeo. S. 
Hassallia Bouteillei Born. et Fl.

Z. S. falaise de Fécamp. D. - Falaise crétacée de Magny-en-Vexin. Bouteille.

Algue aérienne, citée dans les Algues maritimes de Debray.

\section{NOSTOGAGÉES}

Nostoc.

Anabæna.

Nodularia.

Nostoc ellipsosporum Rabh.

= Anabaena maritima ellipsospora Crn.

Palues maritimes, baie de Dinant. CRN. - St-Nazaire. LLord.

Espèce d'eau douce, m'écrit M. Bornet.

N. entophytum Born. et Fl.

$=N$. tenuissimum Born.

Sur d'autres Algues, eaux saumâtres, Tourlaville (Cherbourg). Born et Fu.

N. Linckia Roth. Chauv.

$=$ Rivularia Roth. $-N$. intricatum Menegh. $-N$. verrucosum

Mare saumâtre de Tourlaville, Cherbourg. Thur.

Espèce d'eau douce (Bornet).

Anabæna torulosa Lagerh.

$=$ Sphaerozyga Carmichaelii Crn. Le Jol. Harv. - Cylindrospermum mesoleptum Ktz.

St-Vaast. Herb. Zan. - Z. M. Cherbourg. Le JoL. - Iles anglo-normandes. H. V. H. - Z. M. bane de St. Mare, baie de Laninon. CrN. - Marais salants, le Croisic. FL.

\section{A. variabilis $\mathrm{Ktz}$.}

$=A$. Thwaitesii Crn. Le Jol. - A. oscillarioides Cooke.

Cherbourg. Le Jol. - Penfeld. Prés salés de Landerneau. CRn. 
Nodularia armorica Thur.

Falaises du bourg de Batz au Croisic. Thur.

N. Harveyana Thur.

= Spermosira Thw.

Cherbourg, fossés du fort de Hommet. THuR. - Marais salants, le Croisic. FL.

N. spumigena Mertens.

A. litorea Born. et Fl. (= Nodularia litorea Thur. - Spermosira litorea Ktz.) - En eau saumâtre, Cherbourg. THur. - Prés salés, Landerneau. CRN.

B. major (= Spermosira major Ktz. - Spermosira litorea crassior Rabh.) - Marais salants, le Croisic. FL. 


\section{ORDRE DES CHLOROPHYCÉES}

\section{PROTOCOCGAGÉES}

Prasinocladus.

Gloecystis.

Palmella.

Hæmatococcus.

Chlorocystis.
Chlorella.

Protococcus.

Halosphæra.

Sykidion.

Codiolum.

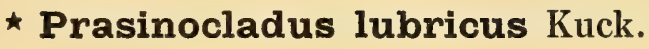

Signalé sur les côtes du Devonshire. Batr.

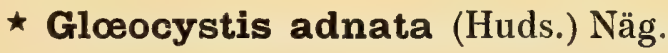

= Tremella Huds. - Palmella Lyngb. - Microcystis Menegh. BatT.

Signalée à Douvres et en d'autres points des côtes anglaises.

\section{G. Paroliniana Näg.}

Falaise $d u$ Blanc-Nez, sur escarpement battu par la mer. De Block. - Base des falaises à Fécamp, Yport, Etretat. D. Antifer, Bruneval, St. Jouin. Bernard.

\section{Palmella conferta Ktz.}

Port-Vieux de Biarritz, sur Rhodochorton floridulum. S.

$$
\text { P. hyalina Rabenh. }
$$

A. rivularis. - Voir Bull. Soc. Bot. de France, mai 1860. 


\section{Palmella litoralis Crn.}

Grève de St. Marc, en bas de la falaise, dans une cavité où la mer entre aux marées. CRN.

P、 mediterranea $\mathrm{Ktz}$.

Banc du Moulin-Blane; base vaseuse des blocs de quartz, bane de St. Marc. CRv.

P. oceanica Crn.

Rivière marine de Penfeld et anse de Kerhuon. CRN.

P. submarina Crn.

Z. S. flaque en face du Moulin-Blanc. CRN.

Hæmatococcus lacustris (Girod.) Rostaf.

$=$ Volvox Girod. - En raison de sa couleur rouge qui attire l'attention, cette espèce porte de très nombreux synonymes.

A. salinus (Dun.) Hansg. (= Protococcus marinus Ktz. Protococcus, Haematococcus salinus Dunal. - Monas Dunalii Joly.) - Côtes de France. Joly.

$\star$ Chlorocystis Cohnii (Wright.) Rke.

= Chlorochytrium Wright.

Indiqué sur la côte $\mathrm{N}$. de la Manche. BatT.

\section{Chlorella...}

Des Chlorelles se développent en abondance dans les bacs de culture (eau de mer) du Laboratoire de Roscoff.

Les Zoochlorelles, qui vivent en symbiose - ou comme parasites - dans les Planaires, se rencontrent partout où se trouve leur hôte; de vastes surfaces de sable (encore humide à marée basse) offrent la Convoluta Roscoffensis à la pointe E. de l'île de Bas; sur l'isthme qui réunit la presqu'île Callot avec Carantec; plage des Basques (Biarritz); au pied de la falaise de Bidart; en face du sanatorium de Hendaye. J. CH.

Protococcus glaucus Crn.

Landerneau. CRN. 
Protococcus magma Bréb. et God.

Baie du Moulin-Blanc et côte N. de Plougastel. CRN.

$\star$ Halosphæra viridis Schm.

Signalée à Plymouth. BATt.

* Sykidion Dyeri Wright.

Sur les Rhizoclonium. Signalé en Angleterre. Batt.

* Codiolum gregarium $\mathrm{A} . \mathrm{Br}$.

Signalé en Angleterre, à Axmouth. BATT.

* G. pusillum Fosl.

$=$ Cod. gregarium et Cod. longipes Holm.

Signalé sur les côtes du Devonshire. Batт.

\section{CONFERVACÉES}

Conferva.

Prasiola.

Pringsheimia.

Protoderma.

Ulvella.

Monostroma.

Enteromorpha.

Ulva.

Gloetila.

Hormiscia.

Ochlochrte.

Acrochæte.
Bolbocoleon.

Pilinia.

Chlorotylium.

Endoderma.

Epicladia.

Tellamia.

Gomontia.

Zygomitus.

Urospora.

Chætomorpha.

Rhizoclonium.

Cladophora.

Conferva bombycina Ag. Chauv.

Se rencontre aussi en eau saumâtre. - Côtes du Calvados. 


\section{Conferva cæspitosa J. Ag.}

Z. S. Argenton. CRN.

\section{C. cannabina Aresch.}

Z. S. Finistère. CRN.

\section{Cladophoræ Crn.}

Rade de Brest, dragué. CrN.

C. curvula (Ktz.) Crn.

$=$ Cladophora refracta Harv. non Ktz. - Cladophora flexuosa Crn. (junior).

Z. I. Penfeld et baie du Moulin-Blanc. Crn.

C. heteronema Ag.

Z. S. Finistère, CrN.

G. retroflexa Bonnem.

$=$ Cladophora Crn.

Finistère, EP. Crn.

Prasiola stipitata Suhr.

$=$ Pr. marina Crn.

Cherbourg. LE Jol. - Sous le château de Brest, Laninon. CRN. Desmaz.

Pringsheimia scutata Rke.

Sur Rhodochorton Rothii, Wimereux. De BLock. - Jersey, sur Chaetomorpha aerea et Phyllophora rubens. 1903. H. V. H.

Protoderma marinum Rke.

Wimereux. De Block.

* Ulvella confluens Rosenv.

Signalée à Weymouth. BAtT.

\section{U. Lens Crn.}

= Phyllactidium Crn.

Dragué rade de Brest. CRN. 
Monostroma fuscum (Post. et Rupr.) Wittr.

= Ulva Post. et Rupr. - Ulva sordida Aresch. - Les Monostroma ont été rangés par les anciens auteurs dans le genre Ulva.

Bords de l'Escaut entre la Tête de Flandre et le Doel ; havre de Philippine; ancien chenal d'Axel. Kx.

\section{Grevillei (Thur.) Wittr.}

= Enteromorpha Thur. - Ulva Le Jol. - Ulva Lactuca Grev. Z. M. Cherbourg. LE JoL. - Z. S. Finistère. Crn.

\section{M. laceratum Thur.}

St. Vaast. LE JoL. - Quineville. Lebet. - Rivière marine de Lauberlac'h et prés salés. CRN.

\section{M. latissimum (Ktz.) Wittr.}

Nieuport, filet d'un pêcheur de Crevettes. Kx. - Quineville. LEBEL.

A. palmatum. - Ostende. W.

M. obscurum (Ktz.) J. Ag.

Sur Corallines, Antifer. Bernard. - Cherbourg. Le Jol. - Sur Stypocaulon, Port-Vieux de Biarritz. S. Welwitsch. - Golfe de Gascogne, sur d'autres Algues. Kтz. in CoL.

M. orbiculatum Thur.

Fossés à Lheure près du Havre. Bernard. - Cherbourg, eaux saumâtres, localité détruite. LE JoL. - Prés salés, Landerneau. CRN. - La Corogne. Laz.

M. oxycoccum (Ktz.) Thur.

$=$ Ulva oxysperma Ktz.

Gatteville, Quineville. Herb. ZAN. - Cherbourg, eaux saumâtres, localité détruite. LE JoL.

M. quaternarium (Ktz.) Desmaz.

$=$ Ulva oxycocca $\mathrm{Ktz}$.

Avelles, Fresney St. Côme, Caen. Len. Bréb. Chauv. - Eaux saumâtres, Tourlaville. Le JoL. - Dans un ruisseau où l'eau entre aux marées, Camfrout (Brest). CRN. 


\section{Monostroma Wittrockii Born.}

Z. S. Cherbourg, mur du quaı Napoléon et avant-port. BorN.

Enteromorpha clathrata (Roth.) J. Ag.

= Conferva Roth. - Ulva Ag.

Eaux de mer et saumâtres, Ostende. W. - Toute la côte du Havre à Dunkerque. D. - Cherbourg. Herb. Zan. - Le Croisic. Fu. - Sur Corallina mediterranea, la Goureppe (Guéthary). S. - San Vicente, Candas. Laz.

A. Agardhiana Le Jol. (=E. Linckiana Grev.) - Iles anglonormandes. H. V. H. - Le Croisic. FL. LE JoL.

B. Agardhiana abbreviata Le Jol. - Z. M. et Z. I. Cherbourg.

C. Agardhiana ambigua Le Jol. - Z. M. et Z. I. Cherbourg. LE JoL. LE JoL.

D. Agardhiana nudiuscula Le Jol. - Z. M. et Z. I. Cherbourg.

E. erecta Le Jol, - Le Croisic. FL.

F. genuina Batt. - Iles anglo-normandes. BATт.

G. Rothiana Le Jol. - Iles anglo-normandes. H. V. H. - Le Croisic. FL.

H. Rothiana foniculacea Le Jol. - Parasite, Cherbourg. LE JoL.

I. Rothiana prostrata Le Jol. - Z Z I. Cherbourg. LE JoL.

J. uncinata tenuis Le Jol. - Cherbourg, EP. plages Napoléon et des Bains, surtout en été. LE JoL.

K. uncinata robusta Le Jol. - EP. plages Napoléon et des Bains, surtout en hiver. Dimensions considérables. LE JoL.

\section{E. compressa (L.) Grév.} Le Jol.

= Ent. complanata Ktz. - Ulva Enteromorpha compressa

Anvers. W. - Ecluse de Heyst, entre les pierres. Kx. - Pierres et pilotis de l'Escaut, entre Calloo et le Doel; polders. Kx. Eure, eaux saumâtres. Herb. Dupray. - Toute la côte de Dunkerque au Havre. D. - Iles anglo-normandes H. V. H. - Roscoff. Sir. Le Conquet. Langeron. - Z. M. Finistère. CRn. - Le Croisic. FL. La Teste-de-Buch. Chantelat. - Biarritz, Guéthary, St. Jean de 
Luz, San Vicente, Rivadeo, la Corogne. S. - Côtes basques, Gijon, le Ferrol. CoL.

A. caespitosa Le Jol. - EP. chenal de Nieuport. Kx. - Cherbourg. Le Jol. - San Vicente de la Barquera, la Franca, Candas. Laz.

B. complanata Le Jol. - Z. M. Cherbourg. Le JoL. - San Vicente de la Barquera. Laz.

C. Cornucopiæ Le Jol. - Z. S. Cherbourg. LE JoL. - Z. S. Toulbroch. CrN.

D. crispa Ktz. - Philippine (Escaut). Kx.

E. falcata Le Jol. - Z. M. Cherbourg. LE JoL.

F. nana J. Ag. - Pilotis du port d'Ostende. Kx. - Murs des quais, Cherbourg. Le Jol. - Z. S. sous le Château de Brest. CRN. - San Vicente de la Barquera. Laz.

G. procerrima Ktz. - Vieux chenal d'Axel. Kx. - Côtes du Calvados. Len.

H. trichodes Ktz. - EP. au Doel. Kx.

Enteromorpha crinita (Roth.) J. Ag.

$=E$. clathrata mucosa Crn. - E. clathrata crinita Hauck. Finistère. CRN.

E. erecta (Lyngb.) J. Ag.

= Ulva clathrata confervoides Ag. - E. clathrata Ktz.

Z. M. Finistère. CrN.

A. plumosa Ktz. - Bane de St. Mare. Crn.

E. flexuosa (Wulf.) J. Ag.

$=E$. Jurgensii Ktz. - Scytosiphon Jurgensii Ag.

Côtes de France. De la Pylaie.

E. intestinalis (L.) Link.

= Ulva Enteromorpha intestinalis Le Jol.

Eau douce en Belgique; canal de Virginal. DE W. - Canal de Louvain. Dr Poskin. - Eaux salées, saumâtres ou douces de la région maritime en Belgique. Kx. - Presque toute la côte de Dunkerque au Havre, surtout en eau saumâtre. D. - Iles anglonormandes. H. V. H. - Le Croisic. FL. - La Teste-de-Buch. Chantelat. - EP. à Biarritz. S. - Fontarabie, le Ferrol. Col. San Vicente, Gijon, la Corogne. S. 
A. bullosa Le Jol. - Eaux saumâtres, Cherbourg. LE JoL.

B. Cornucopiæ (=E. Cornucopiae Carm). - La Franca. LAZ.

C. crispa Le Jol. - Polders, Belgique. Kx. - Cherbourg. LE JoL. - Carantec, île Callot, Roscoff, port de Camaret. J. CH. - La Teste-de-Buch. Chantelat.

D. filiformis. -- Z. S. Kervallon. CRN.

E. flagelliformis Le Jol. - Cherbourg. LE JoL.

F. intestiniformis. - Eau douce et saumâtre, Camfrout, Lauberlac'h. CRN.

G. gigantea. - Entrée du chenal de Nieuport. Kx.

H. maxima Le Jol. - Caen, Oystreham. Chauv. - Eaux saumâtres, Cherbourg. LE JoL.

I. mesenteriformis Ktz. - Escaut à Anvers. W. - Assenede. Coemans. - Ostende. W. - Le Conquet. Langeron. - Eau saumâtre, île de Bas, Plomeur, Penmarc'h. CrN. - San Vicente, Candas, la Corogne. Laz.

J. procerrima Le Jol. - Eaux saumâtres, Cherbourg. LE JoL. Atteint $2 \mathrm{~m}$. de longueur.

K. rivularis. - Embouchure des rivières où remonte le flux ; Finistère. CRN.

L. ventricosa Le Jol. (=E. intestinalis maxima Crn.) - Cherbourg. LE JoL. - Iles anglo-normandes H. V. H.

Enteromorpha lingulata J. Ag.

$=$ E. compressa lingulata Hauck. - Ulva compressa Ag. E. crinita Ktz.

Biarritz, Guéthary, St. Jean de Luz. S.

\section{E. Linza (L.) J. Ag.}

$=$ Phycoseris crispata, Ph. lanceolata, Ph. Linza Ktz. Ulva Enteromorpha lanceolata Le Jol.

Philippine. W. - Port d'Ostende. W. Kx. - Chenal de Nieuport. W. - Iles anglo-normandes. H. V. H. - Z. M. Finistère. Crn. - La Teste-de Buch. Chantelat. - Le Ferrol, la Corogne. Col.

A. crispata J. Ag. - Port de Guéthary. S.

Forme étroite lancéolée ( $=U$. lapathifolia Aresch.) - Rade de Brest. Crn. 
Forme rameuse (= Phycoseris lanceolata ramifera $\mathrm{Ktz}$. ) Rivières marines du Finistère. CRN.

Enteromorpha marginata J. Ag.

Z. S. fossés du port militaire de Cherbourg. LE Jor.

\section{E. micrococca Ktz.}

= Ulva intestinalis micrococca Le Jol.

Cherbourg, murs de clôture des pares aux huîtres. Le JoL. Sur bois flottants, Finistère. CRN. - Biarritz, Guéthary, St. Jean de Luz. S.

\section{E. percursa (Ag.) J. Ag.}

Eaux de mer et saumâtres, Ostende. Kx. - Wimereux, la Rochette. De Block. - Fossés au Havre. Dupray. - Eaux saumâtres, quai Napoléon à Cherbourg. THuR. - Z. M. port Napoléon III, Moulin-Blanc. CrN. - Salines du Croisic. Fu.

E. plumosa $\mathrm{Ktz}$.

$=$ Ulva Rothiana gracilis Le Jol. $-E$. Hopkirkii Crn. $-E$. paradoxa Ktz.

Z. I. plages Napoléon et des bains. Le JoL. - Iles anglo-normandes. H. V. H. - Bane de St. Marc. Cres.

\section{E. prolifera (Muell.) J. Ag.}

$=$ Ulva compressa prolifera Ag. - E. compressa prolifera Grev. - E. intestinalis prolifera Hauck.

Rivages de la mer et embouchures des fleuves, toute l'Europe. De Tonr. - Oystreham, Bernières, Caen. Chauv. - Jersey 1900. H. V. H. - La Corogne. Laz.

\section{E. Ralfsii Harv.}

Rochers de Longlet, fossés des fortifications au N. du port militaire. LE JoL.

E. ramulosa (Engl. Bot.) Hook.

= Ulva clathrata uncinata Le Jol.

Sur les pierres du port d'Ostende. W. - Aquarium du Laboratoire de Wimereux, Pointe aux Oies. De Block. - Cherbourg. Herb. ZAN. - Jersey 1902. H. V. H. - Roscoff. SrR. - Rivage de la mer 
à Bertheaume. CRn. - Le Croisic. Fl. - Biarritz, Guéthary, St. Jean de Luz, San Vicente, Gijon, Rivadeo, la Corogne. S. - Le Ferrol. CoL.

A. robusta Hauck. - Sur Algues et EP. plages Napoléon et des bains à Cherbourg. LE JoL. - Guernesey. BATT. - Bertheaume. CRN.

B. spinescens (=E. spinescens Ktz.) - Jersey 1902. H. V. H.

C. tenuis. - Z. I. Cherbourg. LE JoL.

D. tenuissima. - Iles anglo-normandes, Jersey. H. V. H.

\section{Enteromorpha tubulosa Ktz.}

= Ulva intestinalis capillaris Le Jol.

Eaux saumâtres, Cherbourg. LE JoL.

E. torta Reinb.

$=E$. percursa Harv.

Guernesey. Batr.

\section{Ulva Enteromorpha Le Jol.}

Le Croisic. FL. D.

Voici, d'après Le Jolis, les formes de cette espèce; on trouvera dans Algues marines de Cherbourg, de cet auteur, une synonymie très complète pour chacune d'elles. Plusieurs figurent dans notre Liste sous le nom générique Enteromorpha.

A. lanceolata $=E$. Linza (L.) J. Ag. Cherbourg. Le JoL.
a) latifolia.
Id.
b) undulata.
Id.
c) crispata.
Id.
d) angusta.
Id.
e) plana.
Id.
f) smaragdina.
Id.

B. compressa $=E$. compressa (L.) Grév.
a) complanata.
b) falcata.
c) caespitosa.
d) nana.
e) Cornucopiae $=E$. intestinalis Cornucopiae J. Ag. 
C. intestinalis $=E$. intestinalis (L.) Link.
a) crispa.
b) ventricosa $=E$. intestinalis maxima Crn.
c) maxima.
d) procerrima.
e) bullosa.
f) eapillaris.
g) flagelliformis.
h) micrococca.

Ulva fasciata Delile.

= Ulva latissima palmata Ag.

Finistère. CRN.

\section{U. Lactuca L.}

Banes de Moules à Blankenberghe. Kx. - Z. M. pierres et poutres des jetées, Ostende. W. - Nieuport. Kx. - De Dunkerque à Grandcamp. D. - Z. I. Cherbourg. Le JoL. - Aurigny. Mme Gaudion. - Jersey. Melle Whilte. - Z. I. Ouessant. CRN. [sous le nom de U. rigida Ag. 2 formes; voir Crn. p. 131 et De Toni p. 111]. - Le Croisic. Fl. D. - La Teste-de-Buch. Chantelat. - Biarritz, Guéthary, St. Jean de Luz, San Vicente, Gijon, Rivadeo, la Corogne. S. - La Franca. Laz. - Santander, Candas, Concha de Artedo (Asturies), le Ferrol. Col.

A. genuina Hauck. (=U. Lactuca latissima Ardiss. - U. latis sima L. - U. rigida Ag. - U. Lactuca rigida Ardiss.) - Cherbourg. LE JoL. - Iles normandes, commune partout. BAtт. - Roscoff. Sir. - Le Conquet. Langeron. - Finistère. Crv. - Le Croisic. Fu. San Vicente, Santander, Candas, la Corogne. Laz.

De cette variété, les frères Crouan ont tiré les 5 formes suivantes :

Étroite, lancéolée ( $=U$. lanceolata Crn.) - Finistère. CRN.

Suborbiculaire à bords plissés (= Phycoseris gigantea Ktz.) - Finistère. CRN.

Palmée (= Phycoseris lobata Ktz. - U. latissima palmata Ag. - U. laciniata Wulf.

J. Ag. - Finistère. CRn.

Ramifiée (= Phycoseris ramosa $\mathrm{Ktz}$.) - Finistère. CRN.

Très rabougrie (=U. stipitata Aresch.) - Sur vieux bois flottants, port de Brest. CRN.

D'après Le Jolis, on peut observer de la var. gemuina six formes :

amplissima. - Plage Napoléon.

linearis. - Presque toujours parasite (épiphyte ?). Cherbourg.

Dillenii. - Cherbourg.

contorta. - $I d$.

simplex. - Id.

multifida. - Id. 
B. lacinulata (Ktz.) Hauck. (= Phycoseris Ktz.) - Querqueville. Herb. Zan.

C. lapathifolia (Aresch.) Hauck. (= Ulva lapathifolia Aresch. - Phycoseris lapathifolia Ktz. - Phycoseris Linza Ktz.) - Philippine et Ostende. W. - Chenal de Nieuport. Kx.

D. Myriotrema (Lenorm ?) J. Ag. (= Ulva Myriotrema Crn. Phycoseris Myriotrema Len. - Ulva latissima umbilicalis Ag.) Plage Napoléon et fossés du port militaire. LE JoL. - Z. I. à l'entrée du port militaire de Brest. CRN.

E. rigida De Toni. (= U. Lactuca rigida Le Jol. - Phycoseris rigida Ktz. - U. Lactuca Wulf.) - Mles anglo-normandes. H. V.H. - Roscoff. Sir. - Côtes basques. CoL.

Chauvin dans Algues de Normandie a distribué Ulva bullosa Smith. (=U. Lactuca, var. B. Huds.) - Eau saumâtre, Caen.

\section{Glœotila marina Crn.}

$=$ Ulothrix Crn.

Passage de Plougastel. Crn.

Hormiscia collabens (Ag.) Rabh.

= Ulothrix Thur. - Conferva Ag. - Hormotrichum Ktz.

Sur la chaîne de bois, entrée du port militaire. LE JoL.

H. implexa (Ktz.) De Toni.

= Ulothrix et Hormidium Ktz. - Ulothrix flacca Hauck. Ulothrix Cutleriae (Harv.) Thur. - Lyngbya et Hormotrichum Cutleriae Harv.

Z. S. Equeurdreville; sur les pierres où coule l'eau douce à la limite de la marée. Thur. - Jersey 1902. H. V. H. - Z. S. MoulinBlanc; même habitat qu'à Equeurdreville. CRN.

\section{H. subtilis (Ktz.) De Toni.}

$=$ Ulothrix Ktz.

Z. M. glacis du fort des Flamands. Le JoL. - Moulin-Blanc, Plougastel. CRN.

\section{Ochlochæte dendroides Crn.}

Dragué sur le banc du Bindic, rade de Brest; sur Solieria chordalis. CRN. 


\section{Ochlochæte Hystrix Thw.}

Prés salés, Landerneau. CRN.

* Acrochæte parasitica Oltm.

Dans écorce de Leathesia et Chorda filum. Signalé à Sidmouth par BATT.

\section{* A. repens Pringsh.}

Dans les cellules corticales de Leathesia et Chorda filum. Indiqué à Weymouth. Batr.

\section{Bolbocoleon piliferum Pringsh.}

Dans écorce de Leathesia, Chorda, Mesogloea et autres Algues. - Cherbourg. Le Jol (note manuscr.) - Iles anglo-normandes. H. V. H.

\section{Bryopsis Balbisiana Lamour.}

Trouvé accidentellement à Cherbourg. BoRs.

Pilinia maritima Rosenv.

Sur les Litorines à Guéthary ; sur Patelles, San Vicente. S.

$\star$ P. rimosa $\mathrm{Ktz}$.

Signalé sur la côte anglaise. BATT.

\section{Chlorotylium cataractarum Ktz.}

Pas marine; souvent mouillée par les embruns. - Wimereux, Pointe-aux-Oies ; Arromanches. D.

\section{Endoderma leptochæte Huber.}

Indiqué sur la côte N. de la Manche. BatT.

$$
\star \text { E. viride Rke }
$$

Dans Porphyra, Nitophyllum etc.

Jersey 1904. H. V. H.

\section{$\star$ E. Wittrockii Wille.}

Ordinairement dans Elachista fucicola. Espèce du nord, n'a pas été signalée au sud du pays de Galles. A rechercher néanmoins. 


\section{Epicladia Flustræ Rke.}

Sur Flustra foliacea et autres Bryozoaires; Gris-nez, Audresselles, Wimereux, Grandcamp. D. - Jersey 1903. H. V. H. - Sur Flustra hispida, Sarcochiton gelatinosum etc. à Roscoff, roche du Loup, île de Bas, île Callot. J. CH.

\section{* Tellamia contorta Batt.}

Commun sur la côte N. de la Manche. BATT.

\section{T. intricata Batt.}

Sur Litorines, Rivadeo. S.

Gomontia polyrhiza (Lagerh.) Born. et Fl.

\section{= Codiolum Lagerh.}

Gris-Nez, Wimereux-Croy, cap Antifer, Luc. D. - Normandie. Dangeard. - Jersey 1903. H. V. H. - Roscoff. Born. et Thur. A la pointe des coquilles de Litorina litorea vivante, à Carantec et Perkiridic; sur carapaces vides de Crabes et de Homards entre le Laboratoire de Roscoff et l'île Verte. J. Ch. - Brest. Le Dantec. Sur vieilles coquilles, tests de Crabes etc... le Croisic, Belle-Isle en mer. Chevreux. Fl. - Biarritz, Guéthary, St. Jean de Luz, San Vicente, Peral, Rivadeo, Villavieja, la Corogne. S.

\section{Zygomitus reticulatus Born. et Fl.}

Perforant vieilles coquilles de Mollusques. Côtes de France. Born. et Fu. (op. cit.) Sans localité.

\section{Urospora penicilliformis (Roth.) Aresch.}

= Hormidium flaccum Ktz. - Ulothrix isogona Thur. Hormotrichum speciosum Crn.

Pierres, Varechs et pilotis du port d'Ostende. W. - Retenue du port de Fécamp. Bernard. - Pilotis à l'embouchure de la Seine. Bernard et Duprax. - Arromanches. Herb. Zan. - Z. S. quai Napoléon et quai des Flamands. ThUR. - Z. S. Hommet, digue. LE JoL. - Iles anglo-normandes. H. V. H. - Z. S. rade de Brest; Z. M. Laninon. CRN.

De Toni comprend dans cette forme Ulothrix flacca Thur. Mais Le Jolis et Van Heurck admettent deux espèces. 
Chætomorpha ærea (Dillw.) Ktz.

Z. S. et Z. M. Gris-Nez, Wimereux, la Crèche, Fécamp, Yport. D. - Le Tilleul, Antifer, Bruneval. Bernard. - Z. S. Cherbourg. LE Jol. - Iles anglo-normandes. H. V. H. - Z. M. Ste-Anne, Trégana. CrN. - Andagorria. J. CH. - Guéthary, San Vicente, cap Oriambre; Gijon, Rivadeo, la Corogne. S.

\section{Gh. Cladophoræ Crn.}

Dragué en rade de Brest sur Cladophora pellucida et Microcladia glandulosa. CRN.

Gh. crassa (Ag.) Ktz.

$=$ Ch. torulosa Ktz. - Conferva Linum Harv.

Philippine (Escaut). Kx. - Iles anglo-normandes. H. V. H. Z. S. port militaire de Brest. CRN.

Ch. fibrosa Ktz.

Cherbourg, plage des Bains. Le JoL.

Gh. gracilis Ktz.

= Conferva Kuetaingii Menegh.

EP. plages du quai Napoléon et des Bains. LE JoL. - EP. baie du Moulin-Blanc. CrN.

\section{Gh. Linum (Muell.) Ktz.}

$=$ Rhiroclonium Thur.

EP. Nieuport. Kx. - Z. S. Querqueville. Thur. - Eau saumâtre ; Réville, Quineville. LE JoL. - Cherbourg. Len. - Jersey 1900. H. V. H. - Z. M. baie du Moulin-Blanc, Kervallon, la Villeneuve. CrN. - Salines du Croisic. Fl. - La Teste-de-Buch. Chantelat.

\section{Ch. Melagonium (Web. et Mohr.) Ktz.}

Z. I. Audresselles, Mesnilval. D. - Bénouville, Etretat, Bruneval, St. Jouin. Bernard. - Pointe de Gatteville, près du phare. Le JoL. - Iles anglo-normandes. H. V. H. - Roscoff. SiR.

Gh. minuta Grn.

Dragué, rade de Brest. Crn. 


\section{Chætomorpha tenella Crn.}

Dragué, rade de Brest. Crn.

Gh. tortuosa (Dillw.) Ktz.

$=$ Ch. implexa Holm. et Batt. - Conferva Dillw. - Rhizoclonium capillare $\mathrm{Ktz}$.

Z. S. Querqueville. Thur. - Iles anglo-normandes. H. V. H. Z. M. anse du Minou. CrN. - St. Jean de Luz. Lapeyrère.

Espèce de la Méditerranée, de l'Atlantique du sud et de l'Amérique, non signalée par De Toni sur les côtes W. de France.

\section{Rhizoclonium arenosum (Carm.) Ktz.}

Kervallon. CRN.

\section{Rh. Casparyi Harv.}

Rivière marine de Penfeld. CrN.

\section{Rh. flavicans (Juerg.) Rabh.}

$=$ Conferva implexa Lyngb.

Sur Callithamnion floridulum, Longlet. LE JoL. - Jersey 1901. H. V. H. - Z. S. Penfeld, anse de Kerhuon. CrN.

Cette synonymie est celle de De Toni, Chlorophycées p. 279. Reinbold ne rapporte pas le $R h$. Kochianum Ktz. au $R h$. flavicans (Juerg.) Rabh. Voir Algen des Kieler Föhrde, pages 131 et 132, en texte et notes. Et Batters (p. 15) donne Rh. flovicans $\mathrm{Rabh}_{0}=R h$. arenosum $\mathrm{Ktz}$.

\section{Rh. implexum (Dillw.) Batt.}

= Conferva Dillw. - Rh. tortuosum Ktz. - D'après Batt. n'est pas syn. de Rh. implexum Ktz. - D'après De Toni, pas syn. de Conferva tortuosa Dillw. et Lyngb. ni de Chaetomorpha tortuosa Ktz.

D'après De Toni, sur toutes les côtes... de Belgique (sans localité ni auteur). Mais aucun auteur belge ni herbier ne donne une station belge. - Debrar réunit Rhizoclonium implexum (Dillw.) Batt. avec Chaetomorpha tortuosa Ktz. sous le nom de $R h$. capillare $\mathrm{K}$ tz. Il ajoute comme synonymes: Rh. tortuosum Ktz., Conferva implexa Harv. Et il donne pour localités: Wimereux, bassin de la retenue de Fécamp, Etretat, St. Jouin. - Z. M. quai Napoléon, Querqueville, île Pelée. Le Jol. - Guernesey. Batr. - Roscoff. Sir. - Z. M. parmi les Chondrus, banc du Château, Laninon, 
St. Marc. CrN. - Quais du port du Croisic. FL. - Biarritz, Guéthary, St. Jean de Luz. S.

Rhizoclonium riparium (Roth.) Harv.

$=R h$. litoreum Lyngb.

Embouchure de l'Escaut. Herb. ZaN. - Z. S. pilotis du port d'Ostende. W. - Wimereux, la Rochette; baie d'Authie. D. Eau saumâtre, le Havre. Dupray. - Fossés et remparts du port militaire, quai Napoléon. LE JoL. - Iles anglo-normandes. H. V. H. - Z. M. Porstrein, Laninon. CRN. - Salines du Croisic. FL, - PortVieux de Biarritz, mêlé à Rhodochorton floridulum et à Ectocarpus pusillus. S.

A. Lenormandii Ktz. - Vire. LeN.

Rh. setaceum Ktz.

Havre de Bouchaute. ScHeidweILER.

Cette station semble provisoirement la plus méridionale.

\section{Cladophora albida (Huds.) Ktz.}

$=$ Conferva Huds. - Conferva Neesiorum Ag. - Cl.gracillima Ktz. non Crn. non Harv. - Presque toutes les espèces du genre Cladophora étaient autrefois nommées, par l'un ou l'autre auteur, Conferva.

Z. I. pierres à l'entrée du port d'Ostende. $\mathrm{Kx}$. - Barfleur. LEN. - Z. I. Ste-Anne, Querqueville, Nacqueville, île Pelée. LE JoL. Iles anglo-normandes. H. V. H. - Roscoff. Sir. - Duon. Melles V. et K. - Le Conquet. Langeron. - Z. M. et Z. S. Délec, Melon, Corson, sur Melobesia et Lithothamnion, voisinage des sources. CRN. - Le Croisic FL. - San Vicente de la Barquera. Laz.

A. refracta Thur. $(=C l$. refracta Harv. $)$ - Iles anglo-normandes. H. V. H. Crn.

B. subrefracta Crn. - Côte W., Porspoder, Melon, Argenton.

\section{Gl. $\operatorname{arcta}$ (Dillw.) Ktz.}

$=C l$. polaris Harv.

Etretat, porte d'amont. Bernard. - Z. M. Cherbourg. Le Jol. - Iles anglo-normandes. H. V. H. - Z. M. Finistère. Z. S. entrée du port de Brest. CrN. 


\section{Gladophora Balliana Harv.}

Entre le Pouliguen et le Croisic. D.

\section{C1. Bertolonii Ktz.}

$=C l$. plumosa Ktz.

La Corogne. S.

Gl. catenata (Ag.) Ardiss.

$=$ Conferva Ag. $-\mathrm{Cl}$. prolifera flaccida Ktz.

Santander, le Ferrol. Cor.

Cl. crispata (Roth.) Ktz.

A. squarrosa Grun. - Forme marine d'une espèce qui habite généralement les eaux douces. - Falaise. LEN.

Cl. crystallina (Roth.) Ktz.

$=$ Conferva sericea Lyngb. - $\mathrm{Cl}$. penicillata Lyngb.

Ostende. Kx. - Cherbourg et côtes du Calvados. Len. - Iles anglo-normandes. Jersey 1903. H. V. H. - Roscoff, île Callot. Melles V. et K. - St. Marc, Moulin-Blanc. CRN.

\section{Gl. expansa (Mert.) Ktz.}

Z. S. Longlet. LE JoL. - Baie du Moulin-Blanc. CRN.

A. glomerata Thur. - Z. I. plage Napoléon. LE JoL.

\section{Gl. flexuosa (Griff.) Harv.}

$=C l$. sirocladia $\mathrm{Ktz}$.

Calvados. Len. - Z. S. Hommet, Ste-Anne, Querqueville. Le JoL. - Iles anglo-normandes. Jersey 1902. H. V. H. - Le Minou, Bertheaume, Porspoder. CRN.

\section{Gl. fracta (Dillw.) Ktz.}

A. flavescens ( $=C l$. flavescens Harv. $)$ - Iles anglo-normandes. H. V. H.

B. genuina ( $=C l$. crispata Hassall.) - Eaux saumâtres, Nieuport. Kx. - Embouchure de la rivière de Fécamp. Bernard. Embouchure de la Seine. Bernard, Dupray. - Eaux saumâtres, Cherbourg. LE JoL. - Iles anglo-normandes. H. V. H. - Finistère. 
CrN. - Salines du Croisic. FL. - San Vicente de la Barquera, la Corogne. Laz. - Le Ferrol. Col.

C. marina Hauck. - Environs d'Axel ; Nieuport. Kx. - Guernesey. Batt.

D. Sarniensis H. V. H. (intermédiaire entre les formes genuina et gracilis). - Jersey, grotte des Pirates à Corbière. H. V. H.

\section{Gladophora glaucescens (Griff.) Harv.}

$=C l$. pseudo-sericea Crn.

St. Vaast, Gatteville. Herb. Zan. - Z. M. et Z. I. Cherbourg; murs du quai Napoléon et de la jetée Ouest. Le JoL. - Iles anglonormandes. H. V. H. - Z. M. baie de Bertheaume, le Minou. CRN. - San Vicente de la Barquera. Laz.

A. flexuosa Crn. - Z. S. au Minou, Bertheaume, Porspoder. Crn.

\section{G1. gracilis (Griff.) Ktz.}

= Conferva vadorum Aresch. - Conferva heterochloa Ag.

Z. I. plage du quai Napoléon; EP. plage des bains. LE JoL. St. Marc, passage de Plougastel ; EP. baie du Moulin-Blanc. CRN. San Vicente de la Barquera. Laz.

\section{Cl. Haligeniæ Crn.}

Sur lanières de Saccorhiza bulbosa (= Haligenia); goulet de la rade de Brest. CRN.

\section{Cl. hirta Ktz.}

\section{$=C l$. flexuosa Ktz.}

Z. S. Hommet, Ste-Anne, Querqueville. LE Jol. - Jersey, St. Aubin 1903, échantillon unique. H. V. H.

\section{Cl. Hutchinsiæ (Dillw.) Ktz.}

\section{$=C l$. diffusa Harv.}

Gris-Nez, Wimereux et toutes les côtes de la Seine inférieure. D. - Z. I. nombreuses stations près de Cherbourg. Le JoL. - Banc de l'île de Bas. Melles V. et K. - Le Minou, Bertheaume, Porspoder, Argenton. Crn. - Belle-Isle. Herb. Zan. - La Teste-de-Buch. ChanTElat. - Grande côte (Croisic). D. - Rivadeo. S.

A. distans Ag. - Iles anglo-normandes. H. V. H. - Avec le type. CrN. 
Gladophora lætevirens (Dillw.) Ktz.

Eaux saumâtres et huîtrières, Ostende. W. - Wimereux, toutes les côtes de la Seine inférieure, Luc. D. - Arromanches. Herb. Zan. - Z. M. Cherbourg. Le JoL. - Iles anglo-normandes. H. V. H. Le Conquet. Langeron. - Finistère. CrN. - Le Croisic. FL.

\section{Gl. lanosa (Roth.) Ktz.}

EP. à Ostende. W. - Z. M. Antifer, St. Jouin. Bernard. Cricqueville près de Grandcamp. Bertot. - Z. I. Cherbourg. LE JoL. - Iles anglo-normandes. H. V. H. - Finistère. CrN. - San Vicente. LAz.

A. uncialis (Muell.) Thur. (= Conferva Muell. - Cl. (Spongomorpha) uncialis Ktz.) - Wimereux. J. Сн. - Bénouville, Antifer, Bruneval, St. Jouin. Bernard. - Z. I. Equeurdreville, Querqueville, Nacqueville. LE JoL. - Iles anglo-normandes. H. V. H. - Finistère. CRN.

\section{Gl. Macallana Harv.}

Z. I. Flamands, Hommet, Querqueville, Nacqueville. Le JoL. EP. bane de St. Marc. CrN.

\section{Cl. Magdalenæ Harv.}

Sur Callithamnion floridulum, Hommet. LE JoL. - Iles anglonormandes. H. V. H. - Brest. BoRs.

\section{Cl. Neesiorum Ktz.}

D'après De Toni, espèce distincte de Cl. albida (Huds.) Ktz. Rivadeo, la Corogne. S.

Cl. nitida Ktz.

Iles anglo-normandes. H. V. H. - St. Mare, baie du MoulinBlanc. CRN - Côtes N. du Morbihan. LeN.

\section{Gl. patens $\mathrm{Ktz}$.}

Environs d'Axel; Nieuport. Kx. - Cherbourg, Quineville. Herв. ZAN. - Eaux saumâtres, Kervallon, Loperhet. CRN. 
Cladophora pectiniformis $\mathrm{Ktz}$.

Jersey 1900. H. V. H. - Baie du Moulin-Blanc. Crs. - Morbihan. LEN.

Gl. pellucida (Huds.) Ktz.

Senneville, Fécamp, Yport. D. - Etretat, Bruneval. Bernard. - Port-en-Bessin, Grandcamp. D. - Granville. Herb. ZAN, - Z. I. sur toute la côte de Cherbourg. Le JoL. - Iles anglo-normandes. H. V. H. - Roscoff. SIR. - Duon. J. CH. - Z. I. banc de St. Marc, batterie du Diable, Trégana. CRN. - Le Croisic. Fl. - Biarritz, Guéthary, St. Jean de Luz, San Vicente, Gijon. S. - La Corogne. Laz.

\section{Gl. penicillata $\mathrm{Ktz}$.}

A. lutescens (Ktz.) Ardiss. $(=\mathrm{Cl}$. lutescens $\mathrm{Ktz} .-\mathrm{Cl}$. glomerata flavescens Hauck.) - Eau saumâtre, étang de l'anse de Garreau. CrN.

\section{Cl. prolifera (Roth.) Ktz.}

Sur les jetées à Blankenberghe. Kx. - Granville. Len. - Biarritz, Guéthary, St. Jean de Luz, San Vicente, Gijon. S. Lespinasse.

\section{Cl. purpurascens Lesp.}

Biarritz. Lespinasse.

\section{Cl. rectangularis (Griff.) Harv.}

$=\mathrm{Cl}$. et Conferva Crouanii Chauv.

Plages Napoléon et des Bains, souvent EP. LE JoL. -- EP. St. Mare, Laninon. CrN. - Brest. Len. Chauv.

\section{Cl. refracta (Roth.) Ktz.}

$=C l$. albida refracta Thur. - Conferva fracta maritima Lyngb. - D'après Thuret, à joindre à $\mathrm{Cl}$. albida : toutes les transitions et mêmes localités.

Wimereux, Pointe-aux-Oies; Fécamp. D. - Antifer, St. Jouin. Bernard. - Cherbourg, Barfleur, St. Vaast. Herb. Zan. - Jersey. Melle Turner. - Roscoff. Sir. - EP. à Laber-Benoît, Z. S. à Kervallon. CRN. - San Vicente, la Corogne. S. 


\section{Cladophora repens (J. Ag.) Harv.}

Z. M. Hommet, Querqueville, Nacqueville. Le JoL. - Iles anglonormandes. H. V. H. - Roscoff. Sir. - Z. S. Kervallon, St. Marc, passage de Plougastel. CRN. - Biarritz, Guéthary, St. Jean de Luz. S.

G1. Rudolphiana (Ag.) Harv.

St. Marc. CRN.

\section{Cl. rupestris (L.) Ktz.}

Sur les pierres, jetée d'Ostende et chenal de Nieuport. Kx. EP. à Ostende. De W. Kx. - Gris-Nez; Wimereux, la Rochette; Boulogne, Ault, toutes les côtes de la Seine inférieure et du Calvados. D. - Z. S. Cherbourg. LE JoL. - Iles anglo-normandes. H. V. H. - Roscoff. Sir. - Z. I. le Conquet. Langeron. - Z. M. Finistère. Crn. - Le Croisic. Fl. D. - San Vicente de la Barquera. Laz. Le Ferrol. CoL.

\section{Gl. subpectinata Ktz.}

Kützing, Species p. 397; Tab. Phyc. IV, pl. 7. fig. 2. Forme douteuse.

Jersey. Kтz.

\section{Gl. utriculosa Ktz.}

$=C l$. laetevirens Harv. - $\mathrm{Cl}$. sericea Huds.

Eaux saumâtres, environs d'Axel; Nieuport. Kx. - Querqueville, plage Napoléon. LE JoL. - Jersey 1900. H. V. H. - Ile Callot. J. CH. -- Roscoff. Melles V. et K. - Z. S. Kervallon, Laninon. CRN. - Biarritz, Guéthary, St. Jean de Luz, Gijon. S.

A. distans ( $=\mathrm{Cl}$. distans Crn.) Z. M. Finistère. CrN.

B. falcata (Harv.) De Toni. (= Cl. falcata Harv.) - Iles anglonormandes. H. V. H.

C. letevirens ( $=C l$. laetevirens (Dillw.) Harv. var : glomerata Thur.) - Hommet, Querqueville, Nacqueville. LE JoL. - Iles anglonormandes. H. V. H.

\section{Gl. vaga $\mathrm{Ktz}$.}

Eaux saumâtres, environs d'Ostende. W. et LeN.

Cette espèce a été récoltée à St. Chamas (Bouches-du-Rhône) par CASTAGNE 


\section{$-73-$ \\ SIPHONÉES}

Ostreobium.

Bryopsis.

Derbesia.

Vaucheria.
Codium.

Halimeda.

Valonia.

Ostreobium Queketti Born. et Fl.

Normandie. Dangeard. - Jersey 1903. H. V. H. - Brest. Le Dantec. - Le Croisic. FL. - San Vicente, Peral. S.

Commun dans vieilles coquilles d'huître. D’après Engler-Prantl, dans Anomia Ephippium seulement.

A. roseum Batt. (= Conchocelis rosea Batт.) - Roscoff, roche du Loup; mêlé à Epicladia Flustrae sur Flustra hispida. J. СH. - Jersey 1903. H. V. H.

\section{Bryopsis hypnoides Lamour.}

EP. à Ostende. W. - Huîtrière à Ostende. Mac-Leod. - Fossés du fort au Havre. Duboc. - Granville. Chauv. - Z. M. et Z. I. Flamands, baie Ste-Anne, Querqueville. LE JoL. - Iles anglonormandes. H. V. H. - Roscoff. Sir. - St. Marc, Laninon, le Délec. CrN.

$\star$ A. atlantica J. Ag. (=Br. hypnoides auct.) - Océan atlantique. De Toni.

$\star$ B. prolongata J. Ag. $(=B r$. hypnoides Harv. $)$ - Côtes d'Angleterre. De TonI.

\section{Br. plumosa (Huds.) Ag.}

$=B r$. arbuscula Lamour.

Pieux et pierres, canal de Bruges à Ostende. W. - Z. M. Gris-Nez, Wimereux, Boulogne, le Portel, Fécamp. D. - Yport, Bénouville, Etretat, Antifer, St. Jouin. Bernard. - Arromanches. D. - Bouées du port militaire de Cherbourg. Le Jol. - Iles anglonormandes. H. V. H. - Roscoff. Sir. - Z. S. le Minou. CrN. - Le Croisic. Fl. D. - Le Pila (Teste-de-Buch). Chantelat. - Biarritz, Guéthary, St. Jean de Luz, Rivadeo, la Corogne. S.

A. adriatica Hauck. - Jersey 1900. H. V. H. 
B. pyramidalis Le Jol. - Au-dessous des rochers de Longlet; entrée du port militaire de Cherbourg. Le JoL.

Derbesia Lamourouxii (J. Ag.) Solier.

= Bryopsis Balbisiana Lamourouxii J. Ag. - Bryopsis Balbisiana Lamour.

Z. M. Querqueville. Thur. - Z. I. San Vicente, rochers de la Lin̂̀era. $\mathrm{S}$.

D. repens Crn.

Bane du Moulin-Blanc. CrN.

D. tenuissima (De Not.) Crn.

= Bryopsis De Not. - Derb. marina Solier.

Rochers de Longlet et de Querqueville. LE JoL. - Banc de St. Marc. CrN. - Avec D. Lamourouxii, Z. I. San Vicente, Gijon. S.

* Vaucheria appendiculata (Vauch.) D. C.

Eaux salées près de Lons-le-Saulnier. VAUCHER. - A rechercher dans le voisinage de la mer.

\section{V. aversa Hass.}

$=V$. ornithocephala aversa $\mathrm{Ktz} .-\mathrm{V}$. rostellata $\mathrm{Ktz}$.

Prés salés, Landerneau. CRN.

V. clavata D. C.

Atteinte par ehaque haute mer; Caen. Chauv.

V. dichotoma (L.) Ag.

$=$ Conferva L. - V. salina Ktz.

Eaux saumâtres entre Honfleur et Trouville. Dupray.

A. marina Hauck. (= V. bursata marina Ktz.) - Eau saumâtre, anse de Kerhuon. CRN.

v. litorea Hoffm. et Ag.

Eau saumâtre, près du Havre. Dupray.

V. marina Lyngb.

Chauvin a distribué dans les Algues de Normandie No 63 une 
espèce qu'il attribue à Lyngby dans la table du fascicule, à Agardh sur l'étiquette de l'échantillon; = Derbesia marina (Lyngb.) Kjellm ? Cette dernière est une plante de la mer glaciale arctique!

Chauvin indique comme localité.: embouchure de l'Orne. Et ajoute : rarissime.

Chantelat a trouvé l'espèce à la Teste-de-Buch.

\section{Vaucheria maritima Crn.}

\section{$=\mathrm{V}$. maritima Ktz. ?}

Lieux baignés rarement par la mer (sans doute aux environs de Brest). CRN.

V. ornithocephala Ag.

$=V$. sericea Lyngb. - V. polysperma Hassall.

A. marina ( $=V$. ornithocephala Le Jol.) - Eau saumâtre, St. Vaast. Le JoL.

\section{V. piloboloides Thur.}

$=V$. fucescens $\mathrm{Ktz} .-$ Derbesia marina Crn.

Z. I. Longlet, Hommet, Ste-Anne, Nacqueville. LE JoL. St. Vaast. Thur. - Z. S. banc du Moulin-Blanc, Laninon. CrN.

\section{V. subsimplex Crn.}

Z. M. banc de St. Marc. CRN.

\section{V. synandra Wor.}

Eau saumâtre, embouchure de la Seine. Dupray. - Cherbourg. LE JOL.

\section{Thuretii Wor.}

$=$ V. velutina Ag.

Eau saumâtre, le Havre. Dupray. - Z.S. Kervallon, Ste-Claude, Lauberlac'h. CrN. - Le Croisic. FL.

\section{Codium adhærens (Cabr.) Ag.}

\section{$=$ Cod. difforme Schousb.}

Iles anglo-normandes. Jersey 1903. H. V. H. - Belle-Isle en mer. Bors. - Banc de St. Mare, baie de Bertheaume, Morgat, St. Mathieu. Crv. - Larges surfaces à Guéthary et St. Jean de Luz; la Corogne. S. Laz. 
Codium bursa (L.) Ag.

= Alcyonium L. - Spongodium Lamour. - Agardhia Cabr.

St. Vaast-la-Hougue. Aresch. - Sur les pierres, Granville. Chauv. - Iles anglo-normandes. H. V. H. - Z. I. banc de St. Marc, du Moulin-Blanc, anse de Garreau. CrN. - La Franca, Candas. Laz.

Probablement en épave dans ces diverses localités..... cependant Chauvin dit positivement sur les pierres. Je n'ai jamais vu cette espèce en place.

\section{G. difforme Ktz.}

D'après De Toni $=$ Cod. adhaerens.

Belle-Isle en mer. Bors. -- San Vicente, Gijon, Rivadeo? S.

\section{G. elongatum Ag.}

$=$ Cod. tomentosum elongatum Ardiss.

Le Minou, Porspoder, Argenton. CRN. - La Franca. Laz. St. Sébastion. Bory in Col.

\section{G. polymorphum Crn.}

D'après De Toni, simple forme du C. bursa.

Dragué, rade de Brest, avec le maerl. CRN.

\section{G. spongiosum Harv.}

Rade de Brest, dragué. CrN.

\section{C. tomentosum (Huds.) Stackh.}

= Spongadium dichotomum Lamour. - Agardhia dichotoma Cabr. - Cod. filiforme Mont.

Wimereux, Pointe-aux-Oies; Boulogne, Etretat, Quihot près de Luc, Port-en-Bessin, Grandcamp. D. - Granville. Chauv. - Z. M. et Z. I. Cherbourg. Chauv. - Iles anglo-normandes. H. V. H. - Roscoff. Sir. - Le Conquet. Langeron. - Z. I. banc de St. Mare, Laninon, Ste-Anne. Crn. - Le Croisic. Fl. D. - Biarritz, Guéthary, St. Jean de Luz, San Vicente, Gijon, Rivadeo, la Corogne. S. - Santander, Candas. Cou.

D'après Lazaro les 3 formes suivantes :

A. elongatum. - San Vicente.

B. genuinum. - San Vicente, Candas, la Corogne.

C. implicatum. - San Vicente, la Corogne. 


\section{Halimeda Opuntia De Not.}

= Corallina Tuna Ell. et Sol. - Halimeda Tuna Lamour.

Candas. LAz.

Espèce méditerranéenne.

\section{Valonia ovalis Ag.}

Sur les Lithothamnion, Biarritz. BoRN. 


\section{ORDRE DES PHÉOPHYCÉES}

\section{CUTLÉRIACÉES}

Zanardinia.

Cutleria.
Aglaozonia.

Zonaria.

\section{Zanardinia collaris (Ag.) Crn.}

= Zonaria Ag. - Padina Grev. - Cutleria Zan. - Cutleria adspersa Menegh., non De Not. - Peyssonnelia umbilicata Ktz.

Iles anglo-normandes. H. V. H. - Dragué sur coquilles, au large de Duon. J. Ch. - Dragué, rade de Brest. CrN. - Biarritz, Guéthary, St. Jean de Luz. Lespinasse. - Guéthary. Thur. Guèthary, sur une Araignée de mer. S.

\section{Cutleria adspersa (Roth.) De Not.}

\section{= Zonaria Ag. - Padina Grev.}

Z. I. Biarritz; Arotcha, près de Guéthary. Lespinasse. S. - Ne semble pas dépasser vers le nord l'embouchure de l'Adour, selon Sauvageau. Cependant je trouve dans Herb. Desmaz. un échantillon avec cette mention : dragué, rade de Brest, à une profondeur de $20 \mathrm{~m}$.

\section{C. multifida (Sm.) Grev.}

= Aglaozonia parvula Zan. - Zonaria reptans Crn. - Aglaozonia reptans $\mathrm{Ktz}$.

Quihot. D.-- EP. Lue, Arromanches. Chauv. - Côtes du Calvados. LEN. - Z. I. au-dessous des rochers de Longlet. LE JoL. - Iles anglo-normandes. H. V. H. - Roscoff, filets des pêcheurs. Melles V. et K. - EP. à Perkiridic. J. CH. - Z. I. St. Marc, Laninon. CRN. Morbihan. Herb. J. B. de Bruxelles. - Guéthary. S. - Candas, San Vicente. Laz.

Varie de $\mathbf{5}$ à $30 \mathrm{ctm}$. de hauteur. 


\section{Aglaozonia reptans $\mathrm{Ktz}$.}

$=A$. parvula Zanard. - Zonaria Crn. - Thalle non sexué de Cutleria multifida, signalé comme Aglaozonia dans les stations suivantes :

Senneville, Fécamp, Yport. D. - St. Jouin. Bernard. - Arromanches. Chauv. - Grandcamp. D. - Z. I. parois des rochers, toute la côte de Cherbourg. Le JoL. - Cherbourg, île Pelée. Thur. - Iles anglo-normandes. Jersey 1903. H. V. H. - Sur Mya arenaria, Pecten etc., dragué à Duon (Roscoff). J. CH. - Dragué, rade de Brest; St. Marc, Ste-Anne. Crs. - Guéthary. Lespinasse.

\section{A. melanoidea Sauv.}

Z. I. Guéthary, côte N. de l'Espagne. S.

Thalle non sexué de Cutleria adspersa.

\section{Zonaria flava Ag.}

= Stypopodium Ktz. - Dictyota Tournefortiana Lamour.

Le Ferrol. CoL.

\section{RALFSIÉES}

Ralfsia. Lithoderma. Sorapion.

\section{Ralfsia clavata (Carm.) Farl.}

\section{= Myrionema? Harv. - Linkia Carm.}

Iles anglo-normandes. H. V. H. - Z. M. Moulin-Blane. CrN.

\section{R. disciformis Crn.}

Dragué, rade de Brest. CRN. Espèce douteuse.

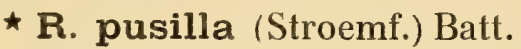

$=$ Stragularia Stroemf.

Sur Laminaria saccharina, côte S. de l'Angleterre. BATT.

* R. spongiocarpa Batt.

Indiqué sur la côte S. de l'Angleterre. BATT. 
Ralfsia verrucosa (Aresch.) J. Ag.

$=R$. deusta Berk. - Zonaria deusta Lyngb. - Padina deusta Hook. - Hildenbrandia deusta Ktz.

Gris-Nez, Audresselles, Wimereux, Fécamp. D. - Antifer. Bernard. - Grandcamp. D. - Z. M. Cherbourg. Le Jol. - Iles anglo-normandes. H. V. H. - Sur Mya arenaria, Roscoff. J. Сн. Z. M. Laninon, sous le Château de Brest. CRN. - Le Croisic. Fu. D. - Sur roches calcaires, St. Jean de Luz. J. Cн.

Lithoderma adriaticum Hauck.

Sur les pierres, dans les pares à huîtres. Tatihou. MaLard et KuCKucK.

L. fatiscens Aresch.

= Ralfsia extensa $\mathrm{Crn}$.

Dragué, rade de Brest. CrN.

* Sorapion simulans Kuck.

= Lithoderma Batt.

Avec Lithoderma fatiscens. Indiqué à Plymouth. BAtT.

\section{LAMINARIÉES}

Laminaria.

Saccorhiza.

Alaria.
Chorda.

Ecklonia.

Phyllaria.

\section{Laminaria Gloustonii Le Jol.}

$=L$. digitata Harv. $-L$. digitata typica et $L$. digitata longifolia Fosl. - L. hyperborea (Gunn.) Fosl.

EP. Wimereux et St. Jouin. D. - Z. I. Cherbourg. Le JoL. Iles anglo-normandes. H. V. H. - Roscoff, ne découvrant qu'aux plus basses mers ; souvent EP. à Perkiridic. J. CH. - Z. I. Finistère. CRN. - Ne découvrant presque jamais, le Croisic. FL. D. - EP. Guéthary, San Vicente, Gijon, Rivadeo, la Corogne. S. - Santander. Laz. 


\section{Laminaria digitata Lamour.}

$=$ L. flexicaulis Le Jol. - L. psendo-digitata Lamour. L. latifolia Aresch.

EP. littoral belge. De W. J. Ch. - Wimereux, Boulogne, de Fécamp au Havre, Quihot, Arromanches, Port-en-Bessin, Grandcamp. D. - Z. I. Cherbourg. Chauv. - Iles anglo-normandes. H. V. H. Iles Chausey. Herb. Zan. - Granville. Chauv. - Roscoff. Sir. Ne découvrant qu'aux plus basses mers et pendant peu de temps. J. CH. - Z. I. Finistère. CrN. - Le Croisic. FL. D. - EP. Guéthary ; en place à San Vicente, Gijon, Rivadeo. S. - La Corogne. Laz. Côtes basques, Santander, le Ferrol. CoL.

\section{L. pallida Grev.}

$=L$. digitata Mont. et Auct.

La Corogne, station septentrionale extrême de l'espèce. S.

\section{L. saccharina Lamour.}

EP. littoral belge. Kx. - Du Blanc-Nez à Boulogne (pas entre Boulogne et Fécamp); de Fécamp au Havre, Quihot, Arromanches, Grandeamp. D. - Z. I. Cherbourg. LE JoL. - Iles anglo-normandes. H. V. H. - Roscoff. Sir. Atteint $7 \mathrm{~m}$. de longueur; souvent dans les mares que le flot laisse en se retirant. J. CH. - Z. I. Finistère. Crn. - Le Croisic. FL. D. - San Vicente, Gijon, Rivadeo, la Corogne. S.

A. bullata Lyngb. - EP. Blankenberghe. Kx.

B. cornea Kx. - EP. Ostende. Kx.

C. latifolia Hook. - EP. Nieuport. Kx.

D. Phyllitis (Stackh.) Hauck. (=L. Phyllitis Lamour. $-L$. debilis Despr.) - EP. Blankenberghe. Kx. - Z. M. Cherbourg. LE JoL. - Iles anglo-normandes. H. V. H. - Z. M. Finistère. CRN. - Plage Valentin (Croisic). D.

Saccorhiza bulbosa (Huds.) De la Pyl.

= Laminaria Lamour. - Haligenia Den.

Au large d'Ostende (fixé?) Landszweert. - Gris-Nez. Moniez. - Cherbourg, îles Chausey. CHAuv. - Iles anglo-normandes. H. V.H. - Environs de Roscoff, ne découvrant qu'aux plus basses mers; mais vivant aussi à la surface, quand l'eau ne l'abandonne pas, sur les bouées flottantes par exemple. J. CH. - Z. I. Finistère. CRN. - 
Le Croisic. Fl. D. - EP. au cap Ferret(Teste-de-Buch). Chantelat. - Biarritz, Guéthary, St. Jean de Luz. S. - Z. M. et Z. I. à St. Jean de Luz et sur toutes les roches de la côte basque; Z. S. dans les flaques ne se vidant pas à basse mer. Au large, par les marées de $5 \mathrm{~m}$. (Socoa), les sommets seulement sont découverts. J. CH. - San Vicente, Gijon, Rivadeo, la Corogne. S. - Cudillero (Asturies), le Ferrol. CoL. - Candas, la Franca. Laz.

\section{Alaria esculenta (Lyngb.) Grev.}

Au large d'Ostende (fixé?) Landszweert. - Digues de Cherbourg. Le JoL. - Iles anglo-normandes. H. V. H. - Roscoff : Duon, station abondante; les Cochons noirs; EP. à Perkiridic. J. CH. Z. I. Corson, Porspoder, Argenton, Melon. Crn. - Au pied de la falaise de Toulinguet (Crozon). J. CH. - Côtes de Galicie. Col.

\section{Ghorda filum (L.) Lamour.}

Implanté sur les brise-lames, Blankenberghe, Ostende et Nieuport. Kx. - EP. à Ostende. W. in Herb. J. B. de Bruxelles. Gris-Nez, Wimereux, Croy. D. - EP. à Onival. De Poli. - Dieppe. D. - Antifer. Bernard. - Quihot. D. - Port-en-Bessin. Chauv. Grandcamp. D. - Z. M. et Z. I. Cherbourg. LE JoL. - Iles anglonormandes. H. V.H. - Roscoff. Sir. Atteint souvent $8 \mathrm{~m}$. de longueur. J. Ch. - Z. I. Finistère. CRN. - Le Croisic. Fu. D. - Gijon (station la plus méridionale). S. - Cependant Cos. indique encore Galicie, Andalousie, Baléares.

A. minor Len. (Etat jeune du type ?) - Filiforme et long seulement de 10 à $15 \mathrm{ctm}$. Portbail (Manche). Len. in Herb. J. B. de Bruxelles. - Herb. Zan.

B. thrix Ag. - Cherbourg. Herb. J. B. de Bruxelles.

\section{Ch. tomentosa Lyngb.}

$=C h$. filum, var. tomentosa Aresch. - Hauck la regarde aussi comme une variété.

Z. I. banc du Château, sous le Polygone, Mingant. CRN.

\section{Ecklonia exasperata (Turn.) J. Ag.}

$=E$. radiata exasperata Turn.

La Corogne. Herb. Thur. 
Phyllaria purpurascens (Ag.) Rostaf.

= Laminaria Ag. - Phyllitis lanceolata Ktz. Un seul exemplaire trouvé en EP. à la Corogne. S.

Phyllaria reniformis (Lamour.) Rostaf.

= Laminaria Lamour. - Haligenia brevipes Lenorm.

Rochers de la Goureppe (Guéthary). Thur. Lespinasse. J. Ch.

- Gijon. S.

\section{SPOROCHNÉES}

Sporochnus.

Carpomitra.

Spermatochnus.
Stilophora.

Halorhiza.

Sporochnus pedunculatus (Huds.) Ag.

EP. à Ostende. W. - EP. à Equihem. Giard. - Luc. D. EP. Cherbourg. LE JoL. - Iles anglo-normandes. H. V. H. - Roscoff, filets des pêcheurs. Melles V. et K. - Laninon. ThuÉBaut. - Dragué, chenal de la rade de Brest; bancs de St. Mare et du Moulin-Blanc, EP. Crn. - San Vicente de la Barquera. Laz. - Le Ferrol. Col.

\section{Carpomitra Gabreræ (Clem.) Ktz.}

Réville. BeNorst. - St. Vaast. AREsch. - Iles anglo-normandes. H. V. H. - Roscoff, dragué à Astan. Melles V. et K. - Dragué, rade de Brest. CRN. - Sur une Araignée de mer à Guéthary. S.

\section{Spermatochnus Lejolisii (Thur.) De Toni.}

= Stilophora Thur. - Sp. microspermus Ktz.

St. Vaast. Thur. - EP. sur Cystoseira, baie Ste-Anne. Le JoL. - Z. M. bane de St. Marc. CRN.

\section{Sp. paradoxus (Roth.) Ktz.}

= Chordaria Lyngb. - Stilophora Lyngbyei J. Ag. - Sporo. chnus rhizodes paradoxus Ag.

Sur Fucus et Zostera, côtes atlantiques d'Europe. De Tons. Valogne (Manche). Dr LeBeL, in HeRB. J. B. de Bruxelles. - St. Mare, 
passage de Plougastel. Crn. - Belle-Isle. Jounan. - Morbihan. Herb. J. B. de Bruxelles.

Stilophora rhizodes (Ehrh.) J. Ag.

= Spermatochnus Ktz. - Sporochnus Ag. - Chordaria Ag.

Luc. D. - Z. M. et Z. I. sur Cystoseira, Cherbourg, Chauv. Iles anglo-normandes. H. V. H. - Granville. Chauv. - Roscoff. Sir. - Z. I. Laninon, Ste-Anne, le Délec. CRN. - Le Croisic. FL. D. Côtes de Galicie. CoL.

Stil. tuberculosa (Horn.) Rke.

= Castagnea Horn. - Chordaria Lyngb. - Stil. papillosa Rke. Cherbourg. De la Chapelle, in Herb. J. B. de Bruxelles.

\section{Halorhiza vaga $\mathrm{Ktz}$.}

$=$ Chordaria tuberculosa Lyngb. - H. tuberculosa Rke.Striaria fragilis J. Ag.

EP. banc de St. Marc. Crn.

\section{GHORDARIÉES}

Chordaria.

Mesogloia.

Castagnea.

Myriocladia.

Microcoryne.

Buff hamia.

Leathesia.

Herponema.

Myrionema.
Eudesme.

Myriactis.

Cylindrocarpus.

Liebmannia.

Cladosiphon.

Phæosphærinm.

Hecatonema.

Chilionema.

Chordaria flagelliformis (Muell.) Ag.

= Scytosiphon hippuroides Lyngb.

Z. M. et Z. I. Cherbourg. Chauv. - Iles anglo-normandes. H.V.H.

- Le Conquet. Langeron. - Z. M. Argenton. Crn.

Les échantillons de Cherbourg atteignent $40 \mathrm{ctm}$., ceux de Guernesey, à peine $5 \mathrm{ctm}$. Herb. J. B. de Bruxelles. 
Mesogloia vermiculata (Engl. Bot.) Le Jol.

$=$ Helminthocladia Harv. - M. lanosa Crn.

Ostende. Herb. J. B. de Bruxelles. - EP. à Nieuport. Kx. - Z. I. Flamands, Ste-Anne, Querqueville. Le JoL. - Iles anglo-normandes. H. V. H. - Granville. Len. - Portbail, St. Malo. Herb. J. B. de Bruxelles. - Le Conquet. Langeron. - Z. I. Laninon, Bertheaume; banc de St. Marc. Crn. - Baie de la Govelle (Croisic). D.

A. coriacea. - Barfleur. Len. in Herb. J. B. de Bruxelles.

\section{Castagnea cæspitosa Thur.}

D'après Bornet, ce n'est qu'une petite forme du Cladosiphon chordariformis. Je la trouve dans Herb. ZaN. sous le nom de Mesogloia caespitosa Auct.

Z. S. Querqueville. LE JoL. - Camaret (dét. Reinbold). J. Сн.

C. contorta Thur.

= Myriocladia Castagnei Crn. - Mesogloia Herb. Zan.

Z. I. plage entre les Bains et le fort des Flamands; St. Vaast. Le Jol. - Z. M. banc de St. Marc. CRN.

C. divaricata (Ag.) J. Ag.

= Chordaria Ag. - Mesogloia divaricata Ktz.

Jersey. Melle White.

G. Griffithsiana (Grev.) J. Ag. Crn.

= Mesogloia Grev. - Helminthocladia Harv. - Myriocladia

EP. à Ostende. W. - St. Vaast-la-Hougue, Portbail. Herb. J. B. de Bruxelles. - Z. I. plage des Bains, Flamands, Ste-Anne. LE JoL. - Carteret. Herb. ZAN. - Iles anglo-normandes. H. V. H. - Roscoff. Sir. - St. Marc, Ste-Anne, Bertheaume; dragué rade de Brest. CrN. - Brest, Belle-Isle, Noirmoutiers. Lloyd.

\section{Myriocladia Zosteræ J. Ag.}

= Mesogloia Aresch.

Cherbourg. Le JoL. - Iles anglo-normandes. H. V. H. - Côtes de Bretagne. Bonnemaison. - Laninon, banc de St. Marc. CRn.

\section{Microcoryne ocellata Stroemf.}

Sur Chorda filum. Signalé près de Weymouth. BATt. 
* Buffhamia speciosa Batt.

Sur Castagnea Griffithsiana. Signalè à Weymouth. BATT.

\section{Leathesia crispa Harv.}

$=L$. concinna Kuck.

Gatteville, sur Chondrus. Malard et Kuckuck. - Iles anglo. normandes. H. V. H.

L. difformis Aresch.

= Nostoc mesentericum Ag. - L. marina J. Ag. - L. tuberiformis Gray.

EP. Wimereux. Giard. - Fécamp, Bénouville, Antifer. Bernard. - Luc, Langrune. Chauv. - Côtes du Calvados. LeN. - Z. M. et Z. I. Cherbourg. LE JoL. - Iles anglo-normandes. H. V. H. - Z. S. Roscoff. J. CH. - Z. M. Finistère. CRN. - Chenal du Croisic. Fu. D. - Z. S. Biarritz, Guéthary, St. Jean de Luz. S. - Couvre de larges surfaces de rocher à Guéthary au printemps; devient très rare en été. J. CH. - San Vicente, Gijon, Rivadeo. S. - Le Ferrol. CoL.

Herponema velutinum (Grev.) J. Ag.

= Sphacelaria Grev. - Elachista Aresch. - Ectocarpus Ktz.

EP. littoral belge, sur Himanthalia. Kx. W. - EP. Wimereux, D. - Bruneval. Bernard. - Luc. Chauv. - Cherbourg, Granville. Chauv. - Jersey et Guernesey. Batт. - Sur Himanthalia, Finistère. Crn. - Belle-Isle. Lloyd. - Le Croisic. FL. - San Vicente, Gijon, Rivadeo, la Corogne. S.

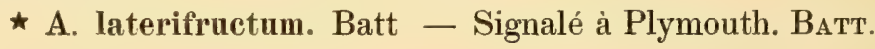

\section{Myrionema Gorunnæ Sauv.}

Le Croisic, sur Laminaria flexicanlis ; la Corogne, sur Laminaria pallida. S.

M. papillosum Sauv.

Sur Laminaria saccharina, Grandcamp. D. - St. Vaast. VANeY. - Le Croisic. Born.

\section{M. polycladum Sauv.}

Sur vieux pieds de Fucus, baie du Toulinguet (Crozon). J. CH. - Sur Fucus serratus, Gijon. S. 
Myrionema strangulans Grev.

$=M$. Leclancherii Harv. Chauv. $-M$. vulgare Thur. $-M$. punctiforme Harv.

Wimereux. D. - Bénouville. Bernard. - Quihot, Arromanches, Port-en-Bessin, Grandcamp. D. - Sur Ulva, Rhodymenia, La Govelle (Croisic). Fu. D. - St. Vaast. S. - Cherbourg. Le Jol. - Iles anglo-normandes. H. V. H. - Granville. Len. - St. Malo. S. - Roscoff sur Ulva. Sir. - Sur Ceramium, Rhodymenia et Ulva, Finistère. Crn. - Belle-Isle. Lloyd. - Biarritz, Guéthary, St. Jean de Luz, San Vicente, Gijon, Rivadeo. S.

Cette espèce varie un peu selon ses différentes plantes nourricières (Thuret). Sauvageau a étudié les échantillons récoltés sur Ulva Lactuca, Enteromorpha compressa et clathrata, Ceramium rubrum.

Eudesme virescens (Carm.) J. Ag.

= Myriocladia Crn. - Mesogloia Zosterae Aresch. - Mesogloia gracilis Berk. - Castagnea Zosterae Thur.

Granville, St. Vaast, le Croisic. Herb. J. B. de Bruxelles. Z. M. Cherbourg. LE JoL. - Iles anglo-normandes. H. V. H. Roscoff. Sir. - Z. M. Finistère. CRN.

\section{Myriactis pulvinata Ktz.}

= Elachista Harv. - Elachista attenuata Harv. - Elachista Rivulariae Suhr.

Langrune, Port-en-Bessin. D. - Cherbourg. LE JoL. - Iles anglo-normandes. H. V. H. - Roscoff. SiR. - Sur Gracilaria confervoides, île de Bas. Melles V. et K. - Sur les Cystoseira, Finistère. Crn. - Le Croisic. Fu. D. - Biarritz, Guéthary, St. Jean de Luz, San Vicente, Gijon, Rivadeo. S.

\section{Gylindrocarpus Berkeleyi (Grév.) Crn.}

\section{= Chaetophora Grev. - Leathesia Harv.}

Hommet, Querqueville, île Pelée. LEJoL. - Iles anglo-normandes. H. V. H. - Z. I. batterie du Diable, Bertheaume. CRN. - Z. I. Arotcha près de Guéthary. S.

\section{G. microscopicus Crn.}

Sur Gracilaria compressa, Finistère. CRN. - Tatihou. MALARD et KUCKUCK. 


\section{Liebmannia gracilis Crn.}

Z. M. baie de Bertheaume. CRN.

\section{Leveillei J. Ag.}

= Mesogloia Menegh. - Mesogloia vermicularis Ardiss.

Z. I. Querqueville, île Pelée. Le JoL. - Iles anglo-normandes. H. V. H. - Roscoff, Kaïnou. J. Сн. - Z. I. le Délec. CRN. - Z. I. Gijon. S.

\section{L. major Crn.}

D'après Batters, $=L$. Leveillei.

Z. I. banc de St. Marc. Atteint 50 cent. de longueur. CRN.

A. gracilior. - Banc de St. Marc. CRN.

Gladosiphon chordariiformis (Crn.) J. Ag.

= Myriocladia Crn. - Castagnea Thur.

Z. M. le Délec, Bertheaume. CRN. - Belle-Isle. Lloyd. - Le Croisic. Fl. - Entre Guéthary et Arotcha; San Vicente, Gijon, Rivadeo. S.

\section{Cl. erythræus J. Ag.}

$=$ Nemacystus Sauv.

Sur Cystoseira ericoides, Z. M. près de la colline de Coroño à Gijon. S.

Phæosphærium punctiforme (Lyngb.) Kjellm.

= Linkia Lyngb. - Myrionema Harv.

Sur Ceramium, Cherbourg. LE JoL. - Sur Ceramium rubrum, îles anglo-normandes. H. V. H. - Sur Ulva, îles Chausey. LEN.

Hecatonema maculans (Collins.) Sauv.

$=$ Phycocelis Collins.

Sur Rhodymenia et Ulva, Grandcamp. D. - Sur Ulva, St. Vaast. S. - Tatihou. Malard et Kuckuck. - Forme différente sur Corallina officinalis, Cherbourg. S. - Sur Rhodymenia, Cherbourg, St. Malo. S. - Roscoff. J. Сн.

\section{Ghilionema Nathaliæ Sauv.}

Sur Rhodymenia palmata, Roscoff. Melle K. 


\section{Ghilionema reptans Sauv.}

$=$ Ectocarpus Crn.

Jersey 1903. H. V. H.

\section{ELACHISTÉES}

Elachista.

Leptonema.
Halothrix.

Giraudia.

\section{Elachista Areschougii Crn.} BoRn.

Sur Himanthalia, banc du Château de Brest. CRN. - Le Croisic.

\section{E. clandestina Crn.}

= Ectocarpus Sauv.

Iles anglo-normandes. H. V.H. - Sur Fucus spiralis, rivière marine de Penfeld. CRN.

E. flaccida (Dillw.) Aresch.

$=E$. curta Aresch. - E. breviarticulata Aresch.

Sur Fucus vesiculosus (fixé ?) et serratus, littoral belge. Kx. Wimereux. Moniez. - Fermanville (Manche). Dr LeBel. - Luc, Carteret, Portbail. HerB. J. B. de Bruxelles. - Cherbourg. Le JoL. - Biarritz, Guéthary, St. Jean de Luz, San Vicente. S. - Iles anglonormandes. H. V. H. -- Roscoff. J. Сн. - La Teste-de-Buch. Chantelat. - Sur Cystoseira, baie de Bertheaume. Crn.

E. fucicola (Vell.) Aresch.

Littoral belge. Kx. - Escaut, Bath près d'Anvers. H. V. H. Ostende. Landszweert. - Wimereux, Boulogne, Petites-Dalles, Fécamp. D. - Bénouville, Antifer. Bernard. - Arromanches, Port-en-Bessin, Grandcamp. D. - St. Vaast-la-Hougue. Len. Cherbourg. LE JoL. - Iles anglo-normandes. H. V. H. - Roscoff. Sir. - Sur les Fucus, Finistère. CRN. - Le Croisic. Fl. - La Teste-de-Buch. Chantelat. - Biarritz, Guéthary, St. Jean de Luz, San Vicente, Gijon, Rivadeo, la Corogne. S. 
Elachista globulosa (Grat.) J. Ag.

= Conferva curta Dillw. De ToN.

Côtes atlantiques de France, sur Cystoseira. A rechercher.

\section{E. Grevillei Arn.}

Sur Cladophora rupestris, mur du quai Napoléon (Cherbourg). THUR.

E. intermedia Crn.

$=E$. curta Crn. - Non : Conferva curta Dillw.

EP, sur Cystoseira granulata, banc de St. Marc. CRN.

E. minutula Reinsch.

Sur Fucus serratus, côtes atlantiques de France. De Tont. A rechercher.

E. scutulata Duby.

EP. sur Himanthalia, littoral belge. Kx. - EP. à Wimèreux et à Langrune. D. - Granville. LEN. - Carteret, Barfleur. Herb. J. B. de Bruxelles. - Cherbourg. Chauv. - Iles anglo-normandes. H.V.H. - Roscoff. Sir. - Sur Himanthalia, Finistère. CRN. - Le Croisic. FL. - La Govelle (Croisic). D. - Un seul exemplaire à Guéthary. S. - Villasellan, Rivadeo. S. - Gijon. Durieu.

E. stellaris Aresch.

$=$ Phycophila Ktz. - Areschougia Menegh.

EP. plage Napoléon à Cherbourg. THur. - EP. sur Arthrocladia villosa, banc de St. Marc, baie du Moulin-Blanc. CRN.

$\star$ A. Chordæ Aresch. - Sur un grand nombre d'Algues. Signalé sur les côtes S. de l'Angleterre. BatT.

\section{E. stellulata Griff.}

= Myrionema J. Ag. - Phycophila Ktz.

Grandcamp. D. - Plage des Bains. Le JoL. - Iles anglo-normandes. H. V. H. - Sur Dictyota dichotoma, baie de Laninon, banc de St. Marc. CRn. - Le Croisic. S. D.

\section{* Leptonema fasciculatum Rke.}

Sur Moules, Ascidies et grandes Algues, côtes d'Angleterre. De Toni. 
* Halothrix lumbricalis (Ktz.) Rke.

= Ectocarpus Ktz. - Elachista Hauck.

Sur Zostère. Trouvé à Weymouth. BATT.

Giraudia sphacelarioides Derb. et Sol.

Plages Napoléon, des Bains, des Flamands. Le JoL. - Sur Zostera et diverses Algues, banc de St. Marc. CRN. - Sur Araignée de mer à Guéthary. S. - Sur Phéosporées parasites, Gijon. S.

\section{PONGTARIÉES}

Dictyosiphon.

Desmarestia.

Arthrocladia.
Stictyosiphon.

Striaria.

\section{* Dictyosiphon Ekmanii Aresch.}

Envahit Scytosiphon lomentarius, comme le Lithosiphon pusilIus (auquel il ressemble beaucoup) envahit le Chorda filum.

Signalé sur la côte S. de l'Angleterre. BatT.

D. fœniculaceus (Huds.) Grev.

= Scytosiphon Ag.

Luc. D. - St. Vaast-la-Hougue. Herb. J. B. de Bruxelles. Herb. Zav. - Z. S. Cherbourg. Chauv. - Iles anglo-normandes. H. V. H. - EP. rade de l'Abervrach. CRN.

A. simplex Suhr. - Cherbourg. Herb. J. B. de Bruxelles.

\section{Desmarestia aculeata (L.) Lamour.}

= Sporochnus Ag.

EP. à Ostende et à Nieuport. Kx. - EP. Bruneval et St. Jouin. Bernard. - EP. Luc, Arromanches. Chauv. - Côtes du Calvados. LEN. - Z. I. Digue, Querqueville; souvent EP. Le JoL. - Iles anglo-normandes. H. V. H. - Roscoff. Sir. Filets des pêcheurs; formes du printemps et de l'automne très différentes. J. Сн. Dragué, rade de Brest et EP. CRN. - Le Croisic. FL. - EP. à Candas et à Rivadeo. S. - Gijon. Durieu. - La Corogne. Laz. 
A, et B. penicillata et fibrillosa LEN. - Ces variétés ne sont que la forme printanière de l'espèce : HerB. J. B. de Bruxelles. La seconde dans Herb. Zan. localité : Barfleur.

\section{Desmarestia Dudresnayi Lamour.}

Cette forme est donnée par De Toni comme simple synonyme de D. ligulata. J'ai vu dans l'Herb. Lenormand plusieurs spécimens qui établissent un vrai passage entre $D$. Dudresnayi et $D$. ligulata. St. Pol de Léon. Dudresnay. - Dragué au large de Roscoff. J. Сн. - Dragué, rade de Brest. CRN.

A. simplex Crn. (=D. pinnatinervia Mont.) - Dragué, rade de Brest à $25 \mathrm{~m}$. CrN.

D. ligulata (Lightf.) Lamour.

= Sporochnus Ag.

Fécamp, Yport. D. - Bénouville. Bernard. - Etretat. D. Bruneval. Bernard. - Grandcamp. D. - Côtes du Calvados. Len. - Z. I. Digue, Querqueville; souvent EP. côtes de la Hague et de Barfleur. LE Jol. - Iles anglo-normandes. H. V. H. - Roscoff. Sir. - EP. à Perkiridic; dépasse parfois $2 \mathrm{~m}$. de longueur. J. CH. - Le Conquet Langeron. - Z. I. et bane de St. Mare, EP. Crn. - Z. I. entre le Sphinx et la Govelle (Croisic). D. - Derrière le musoir du Croisic. Fl. - La Teste-de-Buch. Chantelat. - Z. M. Port-Vieux de Biarritz. J. Cн. - Biarritz, Guéthary, St. Jean de Luz, Gijon, Rivadeo. S. - San Vicente, la Franca, Candas, la Corogne. Laz. Cap Ortegal. CoL.

En été, on ne trouve dans toutes ces stations que des pieds dénudés et dépouillés, réduits aux plus grosses ramifications. LE JoL.

A. angustior Turn. - EP. plus commun que le type, Cherbourg.

\section{D. pinnatinervia Mont.}

D'après J. Ag. c'est le rachis de D. ligulata.

St. Sébastien. Montagne in CoL.

\section{D. viridis (Muell.) Lamour.}

= Sporochnus Ag.

Dieppe. Leturquier. - Z. I. digues; bouées du port militaire; parfois EP. LE JoL. - Iles anglo-normandes. H. V. H. - Portbail 
(Manche). Herв. J. B. de Bruxelles. -- Roscoff, filets des pêcheurs; formes de printemps et d'automne très différentes. Melles $\mathrm{V}$. et K. Z. I. entrée du port de Brest, banc de St. Marc, baie du Moulin-Blanc. Crv. - Environs du cap Ortegal. CoL.

\section{Arthrocladia villosa (Huds.) Duby.}

$=$ Sporochnus Ag. - A. villosa f. australis Hauck.

EP. à Nieuport. W. - EP. Luc. Chauv. - St. Vaast-la-Hougue. Len. - Portbail (Manche). Herb. J. B. de Bruxelles. - EP. Cherbourg. LE JoL. - Iles anglo-normandes. H. V. H. - Roscoff, ramené par les filets des pêcheurs. J. CH. - Banc de St. Marc, Abervrach, St. Pol de Léon, EP. Crn. - Belle-Isle. Desmaz.

\section{Stictyosiphon adriaticus Ktz.}

= Striaria attenuata crinita Auct. (pro parte).

Sur les pierres dans parcs à huîtres. Tatihou. MaLaRd et Kuckuck.

St. Griffithsianus (Le Jol.) Holm, et Batt.

= Phloespora brachiata Born. - Ectocarpus Le Jol. Ectocarpus cruciatus Ktz. Crn.

Barfleur, St. Vaast-la-Hougue. Herb. J. B. de Bruxelles. - Z. I. Cherbourg. LE JoL. - Iles anglo-normandes. H. V. H. - Sur Rhodymenia palmata, Ste-Anne. CRN.

St. subarticulatus (Aresch.) Rke.

$=$ Phloeospora Aresch. - Dictyosiphon foniculaceus subarticulatus Aresch.

Jersey 1904. H. V. H.

\section{Striaria attenuata (Ag.) Grev.}

= Solenia Ag. - Dictyota Grev. - Carmichaelia Grev. Zonaria lineolata Ag.

Iles anglo-normandes. H. V. H. - Laninon, St. Marc. CrN. Morbihan. Heri. J. B. de Bruxelles.

A. fragilis Ag. - Parc à huîtres près de Marennes, Charente infre. Guillon.

Cette variété serait-elle identique à: Striaria fragilis J. Ag. (= Halorhiza vaga.)? 
 \\ PHÉOCAPSÉES}

Phæocystis.

Phæocystis Pouchetii (Hariot.) Lagerh.

= Tetraspora Hariot. - Incertae sedis Engler Prantl.

Z. S. Wimereux et tout le Pas-de-Calais. GIaRd.

\section{ENCÉLIAGÉES}

Microsyphar.

Lithosiphon.

Phloospora.

Punctaria.

Desmotrichum.
Pogotrichum.

Seytosiphon.

Phyllitis.

Colpomenia.

Hydroclathrus.

Asperococcus.

* Microsyphar Porphyræ Kuck.

Indiqué à Folkestone. BatT.

Lithosiphon Laminariæ (Lyngb.) Harv.

= Desmotrichum Ktz. - Punctaria Crn. - Bangia Lyngb.

- Chlorosiphon Harv. - Asperococcus? J. Ag.

Sur Alaria, EP. à Ostende. W. - Côtes de Calvados, Cherbourg. Chauv. - Jersey 1904. H. V. H. - Sur Alaria, Corson. CRN. Sur Saccorhiza, Biarritz, Guéthary, St. Jean de Luz; sur Laminaria Cloustonii, San Vicente. S.

\section{L. pusillus (Carm.) Harv.}

= Asperococcus ? Carm. - Chlorosiphon Harv. - Punctaria Crn. - Chorda filum trichodes Lyngb.

Sur Chorda filum, EP. Wimereux. GIARD. - Cherbourg. Le JoL. - Iles anglo-normandes. H. V.H. - Portbail. Len. - Iles Chausey. Herb. J. B. de Bruxelles. - Roscoff. Sir. - Le Conquet. Langeron. - Finistère. CrN. - Le Croisic, sur Chorda filum. FL. - Gijon. S.

- San Vicente de la Barquera. Laz. 
Phloeospora tortilis (Rupr.) Aresch.

Sur Chorda filum ; Roscoff. J. CH. Dét. Heydrich (" échantillon quoique stérile, absolument certain „).

\section{Punctaria cæspitosa J. Ag.}

Sur Zostera, près de Plouguerneau. CrN.

$$
\text { P. latifolia Grév. }
$$

$=P$. debilis Ktz. - Laminaria debilis Harv.

Z. M. et Z. I. plages Napoléon et des Bains; Equeurdreville. LE JoL. - Iles anglo-normandes. H. V. H. - Ile Callot. Melles V. et K. - Finistère. CRN.

A. dictyotoides. - EP. à la pointe d'Aiguillon (Teste-de-Buch). Chantelat.

B. tenuissima Harv. (= P. tenuissima Harv. - P. latifolia Zosterae Le Jol. - Zonaria plantaginea tenuior Ag.) - Plages Napoléon et des bains à Cherbourg. Le Jol. Herb. J. B. de Bruxelles. - Batterie du Diable à Brest. Herb. Desmaz.

P. plantaginea (Roth.) Grev.

= Ulva Roth. - Zonaria Ag. - Laminaria Ag.

EP. à la Panne. W. - Antifer, Bruneval. Bernard. - Luc. ChaUv. - Cricqueville. Вевтот. - Z. I. plages Napoléon, des Bains, Flamands, Ste-Anne. Le JoL. - Iles anglo-normandes. H. V. H. Anse du Minou. CRs.

A. Crouanii Thur. - Z. I. Hommet, Querqueville, Flamands. LE Jol. - Sur Chorda filum, le Croisic. Fu.

\section{* Desmotrichum balticum Ktz.}

Sur Zostère et sur Algues diverses. Signalé à Weymouth. Batr.

D. cervicorne Ktz.

Dans filaments de Chaetomorpha setacea, Cherbourg. Len.

\section{Laminariæ Ktz.}

= Lithosiphon Laminariae Harv. ?

Querqueville, Dr LEBEL, in HERB. J. B. de Bruxelles. 


\section{D. undulatum (J. Ag.) Rke.}

$=$ Punctaria J. Ag. - Punctaria latifolia Zosterae. Le Jolis? - Punctaria laminarioides Crn.? - Punctaria tenuissima Grev?

Sur Chorda filum, EP. à St. Jouin. BERnARd. - Plages Napoléon et des Bains à Cherbourg. LE Jol. - Anse de Tréménarch en Plouguerneau, Z. I. et EP. CRN.

\section{Asperococcus bullosus Lamour.} Duby.

$=$ Encoelium Ag. - A. Turnerii Hook. - A. rugosus bullosus

EP. à Cricqueville. Bertot. - Barfleur. Len. - Z. I. et EP. plages Napoléon et des Bains. LE JoL. - Iles anglo-normandes. H. V.H. - Roscoff, plage au S. de Ste-Barbe. J. Ch. - Z. I. bane de St. Marc. CRn. - Dans le port du Croisic. Fu. - Morbihan. Herb. J. B. de Bruxelles. - Bords du bassin, la Teste-de-Buch. Chantelat.

\section{A. compressus Griff.}

= Haloglossum Griffithsianum Ktz.

Pêché (?) au large d'Ostende. Landszweert. - Z. M. et Z. I. Flamands, Ste-Anne. LE JoL. - Iles anglo-normandes. H. V. H. Laninon, St. Mare, le Minou. Crn. - Biarritz, Guéthary, St. Jean de Luz. S.

\section{A. echinatus (Mert.) Grev.}

= Encoelium Lyngbyanum Grev. - A. fistulosus Hook.

Z. I. Antifer, Bruneval, St. Jouin. Bernard. - Grandcamp. Chauv. - Barfleur. Len. - Z. M. Cherbourg. Le Jol. - Iles anglonormandes. H. V. H. - Z. M. Finistère. CRN. - Grande côte (Croisic). D.

A. rugosus. - Portbail (Manche). Herb. J. B. de Bruxelles.

B. vermicularis (Griff.) Harv. (=A. vermicularis Griff.) Mêlé au type, Cherbourg. LE JoL. - Finistère. CRN.

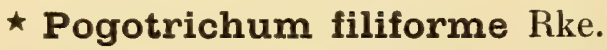

Sur Laminaria saccharina. A été trouvé à Weymouth. BatT.

Scytosiphon lomentarius (Lyngb.) J. Ag.

$=$ Chorda Lyngb. - Chorda filum lomentaria Ktz. - Scyt. fistulosus Ag. 
Sur les pierres, Z. I. port d'Ostende. Herb. J. B. de Bruxelles. - Côtes de Belgique. Kx. Mac-Leod. - Z. S. Wimereux. D. Pointe-aux-Oies, la Rochette et Croy. GIARd. - Yport. D. Bénouville, Etretat, Antifer, St. Jouin. Bernard. - Luc. Chauvin. - Port-en-Bessin. D. - Z. M. Cherbourg. LE Jol. - Atteint à Cherbourg $30 \mathrm{ctm}$. de hauteur. Herb. J. B. de Bruxelles. - Iles anglo-normandes. H. V. H. - Roscoff. SiR. J. CH. - Z. M. Finistère. Crn. - Le Croisic. Fl. - Belle-Isle. Jouan. - La Teste-de-Buch, pointe d'Aiguillon, au Pila. Chantelat. - Biarritz. De 2 à $3 \mathrm{ctm}$. de hauteur. Voyage de l'Unio. - Biarritz, Guéthary, St. Jean de Luz, San Vicente, Gijon, Rivadeo, la Corogne. S.

A. zostericola Thur. - Cherbourg. LE JoL.

\section{* Scytosiphon pygmæus Rke.}

Sur Bryozoaires. Signalé à Weymouth et Swanage. BATT.

\section{Phyllitis Fascia (Muell.) Ktz.}

= Laminaria caespitosa J. Ag. - Ph. caespitosa Le Jol. Ph. Fascia caespitosa Hauck et Richt.

Z. I. sur pilotis du chenal de Nieuport. Kx. - Yport. Bernard. - Côtes du Calvados. Chauv. - Cherbourg, Querqueville. Herb. Z AN. - Iles anglo-normandes. H. V.H. - Laninon, le Délec, Bertheaume. Crs. - Cap Torres, Gijon, la Corogne. S.

A. debilis Hauck. - Guéthary. Lespinasse. S.

B. genuina. - Guernesey. BATT.

$\star$ Ph. filiformis Batt.

Ile de Wight. BatT.

\section{Ph. zosterifolia Rke.}

$=$ Ph. Fascia Le Jol. - Ilea Fascia Aresch.

Cherbourg. LE JoL.

Golpomenia sinuosa (Roth.) Derb. et Sol.

$=$ Hydroclathrus Zan. - Encolium Ag.

Biarritz (EP. ?) Voyage de l'Unio. - Santander, San Vicente de la Barquera, le Ferrol, la Corogne. Laz.

Espèce de l'Adriatique et de l'Atlantique méridionale. 


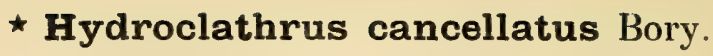

= Asperococcus Endl. - Encoelium Ag. - Stilophora Ag.

Océan atlantique méridional et jusque sur les côtes de Bretagne. Bony. - D'après M. Bornet, la station de Belle-Isle en mer (Bory) est erronée, car les coquilles incluses dans les échantillons de l'Algue ne se rencontrent point sur les côtes bretonnes.

\section{SPHACÉLARIÉES}

Sphacella.

Sphacelaria.

Chaetopteris.
Cladostephus.

Halopteris.

Stypocaulon.

* Sphacella subtilissima Rke.

Envahit Sporochnus pedunculatus et Carpomitra Cabrerae, Plymouth. Batt. - Baléares. Rodrigubz.

\section{Sphacelaria bipinnata Sauv.}

= Stypocaulon Ktz. - Sph. Lebelii Sauv.

Cherbourg, plage des Flamands, rochers du Hommet, baie Ste-Anne sur Halidrys siliquosa. Thur. (récolté par Thuret sous le nom de Sph. cirrosa Ag.) - Carteret, Moitiers d'Allonne. LeBeL (sous le nom de Sph. amphicarpa). - Roscoff sur Halidrys. Melle V. - Brest, sur Halidrys. CRN. (sous le nom de Sph. cirrosa). - Quélern. Le Dantec. - Morbihan. Prouhet (sous le nom de Sph. cirrosa).

Toutes ces stations d'après Sauvageau. De plus : en abondance au pied de la falaise de Pen-Tir (Crozon) sur Halidrys. J. Сн.

\section{S. cæspitula Lyngb.}

Côtes du Calvados. Herb. J. B. de Bruxelles. - Cherbourg. Len. - Carteret. Herb. ZAN. - Sur Saccorhiza, Finistère. Crn.

S. cirrosa (Roth.) Ag. Lyngb.

$=$ Sph. fusca Ag. - Sph. cervicornis Ag. - Sph. pennata 
Sur les Algues du littoral belge (fixé ?) Kx. - Luc. Bernard. - Langrune, Grandcamp. D. - Cherbourg. LE JoL. - Portbail (Manche), Carteret. HerB. J. B. de Bruxelles. - Iles anglo-normandes. H. V. H. - Roscoff. Sir. - Banc de St. Marc. CRN. - Le Conquet. Langeron. - Le Croisic. Fu. D. - Morbihan. Herb. Zan. - La Teste-de-Buch. Chantelat. - Sur Cystoseira ericoides, Biarritz, Guéthary, St. Jean de Luz, San Vicente, Gijon, Rivadeo, la Corogne. S.

A. Aegagropila (Ag.) Wittr. - Granville. HerB. J. B. de Bruxelles.

B. fusca (= Sph. fusca Harv.) - Iles anglo-normandes. H. V. H.

C. nana Griff. - EP. sur Furcellaria, Ostende. W. - EP. Cherbourg. Le Jol. Thur. - Jersey. Melle White. - Carteret. Leber (sous le nom de Sph. cirrosa pygmaea). - Sur Desmarestia aculeata, Finistère. CRN.

D. patentissima Grev. - Cherbourg. Le Jol. - Plage des bains. ThuR. - Normandie (Carteret?) LeBeL. - Roscoff. Melle V. - Brest, St. Marc. Le Dantec.

E. pennata Hauck. - Jersey, Guernesey, Aurigny. BÁtT.

Avant les travaux de M. Sauvageau, le Sph. bipinnata était confondu dans le $S p h$. cirrosa. Donc, parmi les stations citées plus haut, quelques unes se rapportent peut-être à la première de ces deux espèces.

\section{Sphacelaria furcigera Ktz.}

$=$ Sph. rigidula Ktz.

Guéthary, sur substratums variés. S.

* A. saxatilis Kuck. - Signalé à Swanage. BATt.

\section{S. Hystrix Suhr.}

Roscoff. Melle V. - Sur Cystoseira ericoides, Biarritz, Guéthary, San Vicente de la Barquera, Gijon. S.

S. olivacea (Engl. Bot.) Ag.

$=$ Sph. pusilla Ktz. - Conferva Engl. Bot.

Côtes de France. De Toni. - Guernesey, Aurigny. Batt.

$\star$ S. plumigera Holm.

$=$ Sph. plumosa Auct. non Lyngb.

Signalé sur la côte $\mathrm{N}$. de la Manche. Batr. 


\section{Sphacelaria Plumula Zan.}

$=$ Sph. pseudo-plumosa Crn. - Sph. plumosa Menegh.

Dragué, rade de Brest (sous le nom de Chaetopteris, selon Sauv.) Crn. - Concarneau. S. - Sur Araignée de mer à Guéthary. S.

\section{S. radicans (Dillw.) Ag.}

$=$ Sph. cirrosa simplex Ag. - Sph. olivacea radicans J. Ag. - D'après Sauvageau, Sph. radicans Harv.

Sur Ahnfeltia plicata, EP. à Ostende. Kx. - Z. I. Audresselles, Wimereux, Grandcamp. D. - Calvados. Chauv. - Z. I. baie Ste-Anne, Flamands. Le Jol. - Iles anglo-normandes. H. V. H. Z. S. Laninon, anse du Délec. Crn. - Brest, Ste-Anne. Le Dantec. - Belle-Isle. Thur. Lloỹ.

\section{S. sympodicarpa Sauv.} SaUv.

Sur Cystoseira fibrosa, sur coquille de Triton, dragué à Guéthary.

\section{S. tribuloides Menegh.}

$=$ Sph. caespitula Kjellm. - Sph. cervicornis Dene. - Sph. fulva Ktz.

Ile de Ré, Guéthary, San Vicente. S.

Chætopteris plumosa (Lyngb.) Ktz.

= Sphacelaria Lyngb. - Cladostephus Holm. - L'espèce distribuée sous ce nom par Crouan est le Sphacelaria Plumula Zan. (Sauvageau).

Contre la jetée du Croisic. D. - Sur les grandes Algues, la Teste-de-Buch. Chantelat. - San Vicente de la Barquera. Laz. Le Ferrol. CoL.

Ni Sauvageau ni De Toni ne donnent cette espèce sur les côtes françaises. Elle appartient à l'Atlantique du $\mathrm{N}$.

\section{Cladostephus spongiosus (Lightf.) Ag.}

EP. à Blankenberghe et à Ostende. Kx. - Tout le littoral de Dunkerque au Havre. D. - Arromanches, Port-en-Bessin. Chauv. - Z. M. Cherbourg. Le Jol. - Iles anglo-normandes. H. V. H. Roscoff. Sir. - Z. M. Finistère. Crn. - Le Croisic. Fu. D. Notre-Dame d'Arcachon. Chantelat. - Biarritz, Guéthary, St. Jean de Luz, San Vicente, Gijon, Rivadeo, la Corogne. S. - Candas. Laz. 
Cladostephus verticillatus (Lightf.) Ag.

$=C l$. Myriophyllum Ag. - Cl. spongiosus Ktz. non Ag.

EP. à Ostende et Nieuport. Kx. - Senneville, Fécamp, Yport. D. - Antifer, St. Jouin. Bernard. - Quihot, Lue, Langrune, Arromanches, Port-en-Bessin, Grandcamp. D. - Z. I. Cherbourg. LE Jol. - Iles anglo-normandes. H. V. H. - Roscoff. SIR. - Z. I. Finistère. Crs. - Le Croisic. Fu. D. - Cap Ferret, la Teste-de-Buch. Chantelat. - Biarritz, Guéthary, St. Jean de Luz, San Vicente, Gijon, Rivadeo, la Corogne. S. - Concha de Artedo (Asturies), le Ferrol. CoL.

Halopteris filicina (Grat.) Ktz.

$=$ Sphacelaria Ag. - Sph. hypnoides Grev. - Sph. simpliciuscula Ag.

Le Tilleul. Bernard. - Iuc. Chauv. - Iles anglo-normandes. H. V. H. - Roscoff. Sir. - Sur Araignée de mer à Guéthary. S.

A. æstivalis J. Ag. - Z. I. Bertheaume, Laber-Ildut, Lannevez, Combrit. CrN.

B. hiemalis J. Ag. - Z. I. Bertheaume, Combrit. Cre. - Ces deux formes dépendent uniquement de l'âge de la plante.

\section{H. Sertularia (Bonn.) Ktz.}

= Sphacelaria Bonn. - Sph. filicina patens Harv.

Plage des bains à Cherbourg. LE JoL. - Iles anglo-normandes. H. V. H. - Dans Herb. ZaN, sous le nom de Sphacelaria sertularioides Auct. échantillons provenant de Carteret, Portbail et Brest. - Portbail (Manche). Len. - Laninon, Concarneau, Z. M. Z. I. et EP. CRN. - Côte d'Arradon, Morbihan; île aux Moines; St. Gildas. LEBEL.

Stypocaulon scoparium (L.) Ktz.

= Sphacelaria scoparioides, Sph. scoparia, Sph. disticha Lyngb. - Sph. Ulex Bonnem.

EP. à Nieuport. Kx. - Fécamp, Yport. D. - Antifer, St. Jouin. Bernard, - Le Havre. Dupray. - Quihot, Langrune, Grandeamp. D. - Iles anglo-normandes. H. V. H. - Roscoff. SiR. - Le Conquet. Langeron. - Brest. Herb. Zan. - Z. M. et Z. I. Laninon, Concarneau. Crn. - Le Croisic. Fl. D. - La Rochelle. Herb. Zan. - La Teste-de-Buch. Сhantelat. - Biarritz, Guéthary, St. Jean de Luz, 
San Vicente, Gijon, Rivadeo, la Corogne. S. - Candas, la Franca. Laz. - St. Sébastien, Santander, Aviles. CoL.

A. æstivale J. Ag. - Z. I. Cherbourg. LE JoL. - Aurigny. Mme Gaudion. - Guernesey. Melle Lelièvre. - Jersey. Melle White. - Z. I. Finistère. CrN.

B. distichum Len. - Portbail (Manche). Herb. J.B. de Bruxelles.

C. glomeratum. - Biarritz. HerB. J. B. de Bruxelles.

D. hiemale J. Ag. - Portbail (Manche). Len. - Z. I. Cherbourg. LE Jol. - Z. I. Ste-Anne, Bertheaume. CRN.

\section{ECTOGARPÉES}

Pylaiella.

Ectocarpus.

Isthmoplea.

Streblonema.

Ascocyclus.

Acinetospora.

Giffordia.
Sorocarpus.

Strepsithalia.

Phycocoelis.

Dichosporangium.

Phæostroma.

Ulonema.

Myriotrichia.

Pylaiella fulvescens (Schousb.) Born.

= Ectocarpus Thur.

Biarritz. BonN. - Z. S. Guéthary, derrière la jetée; San Vicente, flaques de la Z.S. plusieurs stations; Candas, la Corogne, baie de l'Orsan. S.

\section{P. litoralis (L.) Kjellm.}

= Ectocarpus Ag. - Ectocarpus firmus Aresch.

Iles anglo-normandes. H. V. H. - Roscoff. Melles V. et K. Z. S. Moulin de Penfeld. CrN. - La Teste-de-Buch. Chantelat. Ria à los Rosales, San Vicente; ria à Villavieja, Rivadeo. S. Côtes basques, Gijon, Candas, le Ferrol. Cos.

A. divaricata Kjellm. (= Pylaiella litoralis compacta [L.] Kjellm. - Ectocarpus compactus Ag.) - Yport. Bernard. - Sur Ascophyllum, Cherbourg. Chauv. Le Jol. 
B. firma (Ag.) Kjellm. (= Ectocarpus siliculosus firmus Ag. Ectocarpus firmus J. Ag. - Ectocarpus litoralis Wyatt.) - EP. sur Ascophyllum, Ostende. Mac-Leod. - Tout le littoral, du Havre à Dunkerque. D. - Cap de la Hève (Havre). Dupray. - Arromanches, la Rochelle. Herb. Zan. - Z. M. Cherbourg. Le Jol. Sur Fucus, Finistère. CRN. - Entre le Pouliguen et le Croisic. D.

C. firma typica Kjellm. - Iles anglo-normandos. BATT.

D. major Chauv. - Arromanches. Herb. J. B. de Bruxelles.

E. opposita Kjellm. ( = Ectocarpus brachiatus Ag. - Ectocarpus firmus vernalis Aresch). - Tourlaville. HerB. ZAN. - Cherbourg, localité détruite. Thur. - Jersey. Melle White. - Rivière marine de Penfeld. CRN.

11 est probable que l'Ect. brachiatus Ag. de Melle White est l'Ect. brachiatus Harv. = Phaeospora brachiata Born. = Stictyosiphon Griffithsianus Holm. et Batt.

\section{Ectocarpus abbreviatus Ktz.}

Arromanches, St. Vaast. Herb. Zan. - Arromanches. HerB. J. B. de Bruxelles.

Est-ce bien l'espèce de l'Adriatique? DE TonI.

\section{E. acanthophorus Ktz.}

Rade de Brest; Laninon, EP. CRN.

\section{E. Battersii Born.}

Arromanches. Hohenacker. - Sur Taonia ; Biarritz, Guéthary, St. Jean de Luz. S.

\section{E. brevifructus Lebel.}

Cherbourg. Herb. J. B. de Bruxelles.

\section{E. confervoides (Roth.) Le Jol.}

$=E$. siliculosus Lyngb. pro parte.

Debray réunit sous le nom de $\boldsymbol{E}$. confervoides tous les $\boldsymbol{E}$. siliculosus de Lyngby ; et il distingue 4 formes :

E. confervoides Kjellm.

E. siliculosus Kjellm.

$E$. arctus Ktz.

E. subulatus Ktz.

Kuckuck dans sa monographie considère $\boldsymbol{E}$. confervoides et $\boldsymbol{E}$. siliculosus comme deux espèces; d'après Reinbold, ce sont deux formes à peine séparables. 
Tout le littoral, du Havre à Dunkerque. D. - Iles anglo-normandes. H. V. H. - Le Croisic. FL. - Grande côte (Croisic). D. Biarritz, Guéthary, St. Jean de Luz. S.

A. penicillatus. - Port-Vieux de Biarritz. S.

\section{Ectocarpus confinis Crn.}

Z. S. rivière marine de Penfeld. CRN.

\section{E. crassicaulis Crn.}

Sur Desmarestia aculeata, banc de St. Marc. CRN.

E. crinitus Carm.

Arromanches. Herb. J. B. de Bruxelles. — St. Mare (Brest). CrN.

E. Grouanii Thur.

$=E$. fenestratus Crn.

EP. à Ostende. Herb. J. B. de Bruxelles. - Z. S. baie Ste-Anne, Hommet, Querqueville. LE JoL. - Jersey 1903. H. V. H. - Sur Scytosiphon lomentarius, baie de Bertheaume. CRN. - Le Croisic. FL.

\section{E. distortus Carm.}

Carteret. Herb. Zan. - Sur Zostère, EP. banc de St. Marc; sur Desmarestia aculeata, dragué, rade de Brest. CRN.

\section{E. fasciculatus (Griff.) Harv.}

EP. à Ostende. Mac-Leod. - Wimereux, Croy; Fécamp. D. Bénouville, St. Jouin. Bernard. - Grandcamp. D. - Hommet, Querqueville, Nacqueville. LE JoL. - Iles anglo-normandes. H. V. H. Roscoff. SIR. - Finistère. CRN. - Sur Laminaria et Rhodymenia, le Croisic. FL. - Biarritz, Guéthary, St. Jean de Luz, San Vicente, Gijon, Rivadeo, la Corogne. S.

A. abbreviatus. - Sur Laminaria flexicaulis, le Croisic. BorN.

B. draparnaldioides Crn. - Sur Himanthalia, EP. à Ostende. Herb. J. B. de Bruxelles. - Cherbourg. Le Jol. - Jersey 1902. H. V. H. - Sur Saccorhiza, rade de Brest. CRN.

C. refractus Ktz. - Sur Himanthalia, St. Marc. CRN. 


\section{Ectocarpus globifer Ktz.}

$=E$. pusillus Ktz.

Sur Rhodymenia palmata, Grandcamp. D. - Cherbourg. Herb. J. B. de Bruxelles. - Sur différents supports, plages Napoléon et des Bains, Ste-Anne. Le Jol. - Sur Cutleria multifida, Brest. CRN. - Sur Codium adhaerens, Z. M. et Z. I. Biarritz, Guéthary, St. Jean de Luz ; sur Cystoseira ericoides, Gijon. S.

E. glomeratus Thur.

$=E$. fasciculatus $\mathrm{Ktz} .-E$. congestus $\mathrm{Crn}$. ?

Jersey 1902. H. V. H. - Hommet, Querqueville, Nacqueville. Le Jol. - Bertheaume. CRN.

E. granulosus (Engl. Bot.) Ag.

$=E$. secundatus Suhr. - E. laetus Ag.

Pierres du port d'Ostende. Mac-Leod. W. - Wimereux, Pointe-aux-Oies et Croy; le Portel, le Tréport. D. - Le Havre. Dupray. - Arromanches, Cherbourg. Herb. Zan. - Z. M. et Z. I. Cherbourg. LE JoL. - Iles anglo-normandes. H. V. H. - Roscoff. Sir. - Ste-Barbe. Melles V. et K. - Z. I. entrée du port de Brest. Crn. - Le Croisic. Fl. - Biarritz, Guéthary, St. Jean de Luz, San Vicente, Gijon (cap Torres), Rivadeo, la Corogne. S.

\section{E. Hincksiæ Harv.}

Hommet, Querqueville, digue. Le JoL. - Sur Saccorhiza, baie de Laninon, Ste-Anne. CrN. - Le Croisic. Fl. - Biarritz. Thur. Sur Saccorhiza et Cystoseira: Biarritz, Guéthary, St. Jean de Luz; sur Laminaria Cloustonii : San Vicente, la Corogne. S.

$\star$ E. Holmesii Batt.

A été signalé sur les côtes $\mathrm{S}$. de l'Angleterre. BAtт.

E. insignis Crn.

Espèce de Crouan. - De Toni la donne p. 539 du Sylloge, tome III, comme syn. de E. globifer; et p. 564 comme espèce distincte. - D'après Sauvageau $=E$. globifer Ktz. et $E$. insignis Crn. Exsiccata no 14. - Hauck réunit E. pusillus Griff., E. pusillus Ktz. (Tab. Phyc.) et E. globifer.

Sur Cutleria multifida, côtes atlantiques de France. CRN. Lloyd. - Cherbourg. Herb. Zan. 
A. minimus Crn. (= Ect. pulchellus Oltm). - Sur Chorda lomentaria. Brest. CRN.

\section{Ectocarpus Landsburgii Harv. (non Dick.)}

L'Ectocarpus Landsburgii Dick. = Pylaiella litoralis varia (Kjellm) Kuck. forme arctique.

Cherbourg. LE JoL. - Sur Desmarestia aculeata, EP. baio du Moulin-Blanc et St. Marc. CrN.

\section{E. lanosus $\mathrm{Ktz}$.}

Côtes du Calvados. LEN.

\section{E. longifructus Harv.}

Cherbourg. Herb. Zan. - Jersey. Melle White. - Entrée du port de Brest. CRN.

\section{E. macrocarpus Crn.}

Sur Cystoseira granulata, côtes du Finistère. CRN.

Est-ce la même espèce qui a été trouvée à la Guadeloupe ?

\section{E. major Ktz.}

$=E$. litoralis var. $\beta$ Lyngb.

Valognes (Manche). Dr LeBeL.

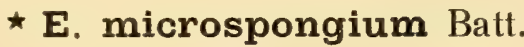

Sur Ralfsia verrucosa. Signalé à Plymouth. Batt.

E. myriocarpus Crn.

Sur Fucus platycarpus, rivière marine de Penfeld. CRN.

$\star$ E. ovatus Kjellm.

A. arachnoideus Rke. - A été trouvé sur la côte S. de l'Angleterre. BATT.

\section{E. parvulus $\mathrm{Ktz}$.}

Arromanches. Herb. J. B. de Bruxelles. Herb. Zan. Espèce de l'Adriatique. 


\section{Ectocarpus Sandrianus Zan.}

$=E$. elegans Thur. - E. laetus Ktz.

Z. I. plages de la jetée de l'Ouest et des Bains. Le Jol. - Sur Polysiphonia elongata, port Napoléon III. CRN.

\section{E. scorpioides Harv.}

Prés salés, Landerneau, sur Obione portulacoides. CRN.

Jen'ai pas trouvé mention de cette espèce ailleurs que dans Crouan. La station est absolument celle du Bostrychia.

\section{E. siliculosus (Dillw.) Lyngb.}

$=E$. amphibius Harv. - E. siliculosus Lyngb. pro parte. E. viridis Harv.

Sur pilotis des ports d'Ostende et de Nieuport. W. - Wimereux, Antifer. D. - St. Vaast. Herb. ZaN. - Arromanches, Cherbourg, Granville, St. Malo. Herb. J. B. de Bruxelles. - Z. M. Cherbourg. Le JoL. - Mes anglo-normandes. H. V. H. - Roscoff. SiR. - Dragué à Astan. Melles V. et K. - Finistère, Z. M. sur Himanthalia; prés salés de Landerneau sur Zostera. CRN. - Le Croisic. Lloyd. - La Teste-de-Buch. Chantelat. - Biarritz, Guéthary, sur Saccorhiza; St. Jean de Luz; sur Laurencia obtusa, Rivadeo. S. - La Corogne. LAz.

A. arctus (Ktz). Kuck. (=E. confervoides arctus Kjellm. - E. pseudo-siticulosus Crn. - Batters rapporte cette forme à $E$. confervoides; De Toni la place dans $E$. siliculosus). - Wimereux, Grandcamp. D. - St. Vaast. Le JoL. - Iles anglo-normandes. H.V.H. - Roscoff. Sir. - Laninon, St. Marc. CrN. - Port du Croisic. D.

B. hiemalis (Crn.) Kuck. (=E. hiemalis Crn. - E. confervoides hiemalis Kjellm.) - St. Vaast-la-Hougue. Dr Lebez. - Plages de la jetée de l'Ouest, des Bains et des Flamands. Le JoL. - Entrée du port de Brest. CRN.

C. longipes Harv. - Jersey. Melle White.

D. subulatus (Ktz.) Hauck. (=E. amphibius Harv.) - Fossés du fort des Neiges au Havre. Dupray. - Jersey 1900. H. V. H.

E. typicus Kjellm. - Jersey, Guernesey, Aurigny. Batт.

\section{E. simplex Crn.}

$=E$. Codii Lloyd.

Hommet, Querqueville. LE JoL. - Roscoff. SIR. - Sur les 
Codium, banc de St. Marc, Ste-Anne, Melon. Crv. - Le Croisic. Ft.

- Biarritz, Guéthary, St. Jean de Luz. S.

\section{Ectocarpus simpliciusculus Ag.}

$=$ Ect. irregularis Ktz.

Cherbourg. Herb. Zan. - Jersey 1902. H. V. H. - Le Croisic. FL. - Biarritz, Guéthary, St. Jean de Luz, San Vicente, Gijon. S.

E. sphacelarioides Crn.

Carteret. Herb. ZAN. - Sur Desmarestia aculeata, banc de St. Marc, EP. CRN.

\section{E. squarrosus $\mathrm{Ktz}$.}

Sur Fucus vesiculosus, côtes du Calvados. De TonI.

$\star$ E. terminalis $\mathrm{Ktz}$.

$=E$. pygmaeus Aresch. pro parte.

Signalé sur les côtes $\mathrm{N}$. de la Manche. BatT.

E. tomentosus (Huds.) Lyngb.

EP. à Ostende. W. - Fécamp. Bernard. - Z. M. et Z. I. Cherbourg. LE JoL. - Iles anglo-normandes. H. V. H. - Ile Callot. Melles V. et K. - Pointe S. de l'île de Bas. J. CH. - Le Conquet. Langeron. - Sur Fucus, Z. M. anse du Délec, baie de Bertheaume. Crn. - Grande côte (Croisic). D. - Au Pila (Teste-de-Buch). Chantelat. - Biarritz, Guéthary, St. Jean de Luz. S. - San Vicente, la Corogne. Laz. - Le Ferrol. Col.

\section{E. uncinatus Crn.}

L'E. uncinatus Ktz. est une autre espèce, signalée à l'embouchure du Douro en Portugal.

Sur Himanthalia, St. Mare. CRN.

\section{E. virescens Thur.}

$=E$. Mitchellae Harv.

Sur pierres, parcs aux huîtres, Tatihou. Malard et Kuckuck. - St. Vaast. Thur. - Cherbourg, Carteret. Herb. ZAN. - Guernesey. Batt. - Belle-Isle. Thur. - Le Croisic. Thur. FL. Morbihan. Lloyd, - Biarritz, St. Jean de Luz. S. - Derrière la 
jetée de Guéthary. Thur. - San Vicente, Gijon, Rivadeo, la Corogne. S.

Isthmoplea sphærophora (Carm.) Kjellm.

= Ectocarpus Carm. - Ectocarpus cruciatus Ag.

Z. M. musoirs des jetées du port du Commerce, à Cherbourg, sur Cladophora rupestris. LE JoL. - Iles anglo-normandes. H. V. H. - Sur Ptilota elegans, anses du Minou et du Mingant. CRN.

^ Streblonema æcidioides (Rosenv.) Fosl.

= Myrionema Sauv. - Ectocarpus Rosenv. BatT.

Signalé sur la côte S. de l'Angleterre, parasite des Laminaria.

S. æquale Oltm.

= Phaeostroma Kuck.

Dans écorce de Chorda filum; Tatihou. Malard et Kuckuck.

S. breve (Sauv.) De Toni.

Sur Laminaria, Ascophyllum etc. Audresselles. D. - Fouras (Charente inf.) Marchand.

S. fasciculatum Thur.

= Ectocarpus Pringsheimii Rke. - Str. volubile Pringsh.

Sur Eudesme virescens, Hommet. LE JoL. - Iles anglo-normandes. H. V. H.

\section{S. infestians Batt.}

\section{= Endodictyon Gran.}

Dans masse gélatineuse de Flustra hispida, Roche du Loup, Roscoff; au pied de la falaise de Pen-Tir (Crozon). Dét. Heydrich. J. Сн.

* S. intestinum Holm. et Batt.

Sur Brongniartella byssoides. Signalé à Weymouth. BATT.

\section{S. investiens Thur.}

$=$ Ectocarpus Hauck.

Sur Gracilaria compressa et multipartita, St. Vaast. LE JoL. - Le Croisic. Fu. S. - Sur Gracilaria compressa, Grandcamp. D. 
Streblonema luteolum (Sauv.) De Toni.

Sur très vieux stipes de Fucus, au Croisic. S. - Iles anglonormandes. Jersey 1904. H. V. H.

S. Myriocladiæ (Crn.) De Toni.

= Ectocarpus Crn.

Sur Castagnea Griffithsiana, banc de St. Marc. Crn.

* S. minimum (Näg.) De Toni.

= Ectocarpus Näg.

Dans réceptacles d'Himanthalia ; signalé à Douvres. BATT.

S. parasiticum (Sauv.) De Toni.

$=$ Ectocarpus Sauv.

Sur Cystoclonium purpurascens, Gracilaria confervoides et Ceramium rubrum; le Croisic. S.

$\star \mathbf{S}$. reptans (Kjellm.) Farl.

= Ectocarpus Kjellm. - Ect. repens Rke. non Crn.

A été signalé dans la Méditerranée, sur les côtes S. de l'Angleterre et en Norvège.

S. solitarium (Sauv.) De Toni.

Dans thalles âgés des Dictyotées, invisible à l'œeil nu ; Port-en. Bessin. D. - Le Croisic, San Vicente. S.

S. sphæricum (Derb. et Sol.) Thur.

$=$ Ectocarpus Derb. et Sol.

Sur Liebmannia Leveillei, Querqueville. Thur. - Sur Nemacystus erythraeus, Gijon. S.

S. Stilophoræ (Crn.) De Toni.

= Ectocarpus Crn.

Sur Stilophora rhizodes, bane de St. Mare. CrN. - Nles anglonormandes. H. V. H.

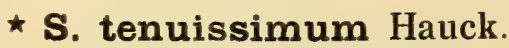

Sur Mesogloia Griffithsiana.

Signalé à Torquay et Swanage. BATT. 
৯ Streblonema tomentosoides (Farlow.) De Toni.

= Ectocarpus Farl.

Sur Laminaria saccharina; signalé à Weymouth. BATT.

S. Valiantei (Born.) De Toni.

= Ectocarpus Born.

Iles anglo-normandes. H. V.H. - Cobo (Guernesey). Mme HaMber. - Sur Cystoseira ericoides, Biarritz. BoRs. - Guéthary, St. Jean de Luz, San Vicente, Gijon, la Corogne. S.

S. volubile (Crn.) Thur.

$=$ Ectocarpus Crn. - Cylindrocarpus Crn.

St. Vaast. Le Jol. - Sur Dudresnaya [coccinea, dragué, rade de Brest. CrN.

S. Zanardinii (Crn.) De Toni.

$=$ Ectocarpus Crn.

Iles anglo-normandes. Jersey 1903. H. V. H. - Sur Lomentaria kaliformis, banc de St. Marc. CRN.

* Ascocyclus balticus Rke.

Signalé à Weymouth. BATT.

\section{A. hispanicus Sauv.}

Sur carapace et pattes d'Araignée de mer à Guéthary; sur Saccorhiza bulbosa, Fucus serratus ot Himanthalia lorea âgés, Rivadeo. S.

\section{A. orbicularis (J. Ag.) Magn.}

\section{$=$ Myrionema J. Ag.}

Sur Zostère, Tatihou. MALARd et Kuckuck. - Cherbourg. Le Jol. - Ies anglo-normandes. H. V. H. - Sur Zostère, dragué, rade de Brest. CRN. - Le Croisic. FL.

\section{A. sphærophorus Sauv.}

Sur Rhodymenia palmata, St. Vaast. Gonont. VANEY. - Cherbourg. Thur. - Iles anglo-normandes. H. V. H. - St. Malo. Born. - Roscoff. Melle K. 
Acinetospora pusilla (Griff.) Born.

\section{$=$ Ectocarpus Griff. - Ect. ostendensis Crn.}

Ostende. Askenasy. - Z. S. Hommet, Querqueville. Le Jol. Iles anglo-normandes. H. V. H. - Sur Corallina officinalis, Z. M. batteries du Diable et du Minou. CrN. - Biarritz. Born. - San Vicente, cap Oriambre, la Corogne [limite S. de l'espèce]. S.

Bornet y distingue les 4 formes suivantes :

A. Codii. - Sur Codium tomentosum, Biarritz, Guéthary; sur Cod. adhaerens, Guéthary. S.

B. riparia. - Avec Rhodochorton floridulum, au pied des falaises du Port-Vieux de Biarritz, sur Polysiphonia macrocarpa et obscura. S.

C. Thuretii. - Sur Nemalion et Helminthocladia, Biarritz, Guéthary. S.

D. typica. - Sur Corallina. C'est la forme figurée par Harvey et par Bornet; la plante des Exsiccata Crouan ( ${ }^{\circ}$ 15), Le Jolis, Desmazières (nº 422). - Cherbourg, St. Malo, Brest. Herв. Thur.

\section{Giffordia fenestrata (Berk.) Batt.}

= Ectocarpus Berk. non Crn.

Sur Scytosiphon lomentarius, EP. Ostende. W. - Z. S. Kervallon. CRN.

\section{G. Lebelii (Crn.) Batt.}

= Ectocarpus Crn. - Elachista Aresch. - D'après De Toni, cette espèce est peut-être identique à la précédente.

Iles anglo-normandes. Jersey 1902. H. V. H. - Sur vieux pieds de Fucus, baie du Toulinguet (Crozon). J. Сн. - Sur Cystoseira, Finistère. CrN. - Biarritz. BonN. - Guéthary, San Vicente, Gijon, la Corogne. S.

\section{G. Padinæ Buffh.}

Sur Padina Pavonia, Biarritz, Guéthary, St. Jean de Luz. S.

G. secunda (Ktz.) Batt.

$=$ Ectocarpus $\mathrm{Ktz}$.

Gris-Nez; Wimereux, Croy ; Fécamp, Port-en-Bessin. D. Cherbourg. JANCZEwski. - Sur Alaria à Cherbourg. Note manuser. Le Jol. - St. Malo. BonN. - Sur Polysiphonia Brodiaei, port 
Napoléon III. Crs. - Sur Saccorhiza, le Croisic. J. CH. - Biarritz. Born. Ktz. - Sur vieux pieds de Saccorhiza bulbosa, Guéthary, St. Jean de Luz, San Vicente, Gijon; sur Himanthalia à Rivadeo ; la Corogne. S.

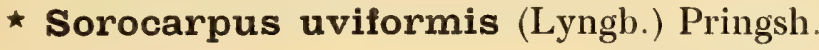

$=$ Ectocarpus siliculosus uviformis Lyngb.

A été trouvé à Weymouth. BATT.

\section{Strepsithalia curvata Sauv.}

A été d'abord distribué par M. Bornet sous le nom de Herponema ambiguum, puis de Phaeocelis ambigua. ThUR.

Dans le parenchyme d'Helminthocladia purpurea, Guéthary.

\section{* S. Buffhamiana Batt.}

= Streblonema Batt.

Parasite sur les Mesogloia Griffithsiana et vermiculata; indiqué à Swanage. BatT.

\section{S. Liagoræ Sauv.}

Sur Liagora et Helminthocladia, Guéthary. Thur.

Phycocelis reptans (Crn.) Kjellm.

= Ectocarpus Crn. - Ascocyclus Rke. - Myrionema Fosl.

Port (Calvados). Herb. ZaN. - Jersey, sur Desmarestia aculeata. H. V.H. - Sur Fucus serratus, bancs du Moulin-Blanc et de St. Marc; Ste-Anne. CRN. - Le Croisic. FL.

Ph. ocellata (Ktz.) Fosl.

= Ascocyclus Rke. - Myrionema Ktz.

Sur Ulva latissima, Portbail (Manche). HerB. J. B. de Bruxelles. - Jersey. H. V. H. - Granville. Herb. Z

* Dichosporangium repens Hauck.

$=$ Myriotrichia Hauck. BatT.

Sur Liebmannia Leveillei. Indiqué sur la côte N. de la Manche. 
* Phæostroma pustulosum Kuck.

Sur Zostera. Indiqué sur les côtes N. de la Manche. BatT.

\section{Ulonema rhizophorum Fosl.}

Jersey 1903, sur Dumontia filiformis. H. V. H. - Tatihou. MaLaRd et KuCKuck.

\section{Myriotrichia claviformis Harv.}

Fermanville. Herb. Zan. - Cherbourg. Le Jol. - Iles anglo. normandes. H. V. H. - Roscoff. J. CH. (Dét. Heydrich). - Sur Scytosiphon lomentarius, Finistère. CRN.

$\star$ M. densa Batt.

$=M$. claviformis Buffh.

Sur Zostera. Trouvé sur la côte sud d'Angleterre. BATT.

M. filiformis (Griff.) Harv.

$=$ M. claviformis filiformis Kjellm.

Arromanches, Cherbourg, Brest. HeRB. ZaN. - Iles anglonormandes. H. V. H. - Roscoff. Melles V. et K. - Belle-Isle. Jouan. - De quelques millimètres sur Castagnea et Nemacystus, Gijon. S.

- San Vicente. Laz.

Ordinairement mêlé à M. claviformis Harv.

\section{TILOPTÉRIDÉES}

Tilopteris.

\section{Tilopteris Mertensii (Smith.) Ktz.}

= Sphacelaria Ag. - Ectocarpus Ag.

St. Jouin. Bernard. - Luc. Chauv. - Z. I. Flamands, baie Ste-Anne, Hommet. LE JoL. - Iles anglo-normandes. H. V. H. Z. I. entrée du port de Brest; limite sud de l'espèce. CrN. 


\section{FUGACÉES}

Fucus.

Ascophyllum.

Pelvetia.

Bifurcaria.
Himanthalia.

Halidrys.

Cystoseira.

Sargassum.

Fucus axillaris J. Ag.

$=F$. vesiculosus axillaris.

San Vicente, Gijon. S.

A. spiralis J. Ag. (=F. vesiculosus lutarius Sauv. $-F$. vesiculosus monocystus Ag. - F. lutarius Ktz.) - Nles Chausey. Chauv. - Ria de San Vicente. S.

\section{F. ceranoides L.}

Ostende, la Panne (EP. ?) Kx. - Recherche l'eau saumâtre, ou l'eau douce intermittente; arrière-port de Dieppe. Desmaz. Retenue de Fécamp. D. - Yport. Bernard. - Port-en-Bessin. Chauv. Z. S. Z.M. et eaux saumâtres; canal de la Divette et au pied des falaises de la Hague. LE JoL. - Iles anglo-normandes. H. V. H. Roscoff. Sir. - Pempoul, à l'embouchure du ruisseau. J. CH. Finistère. CRN. - Abondant sur les murs des quais atteints par la marée, à l'intérieur des villes de Landerneau, Quimper, Bayonne. J. Сн. - Bords du bassin d'Arcachon. Сhantelat. - Embouchure de l'Adour. S. - St. Jean de Luz, embouchure de la Nivelle; embouchure de la Bidassoa, entre Hendaye et Fontarabie. J. Сн. - Côte S. du golfe de Gascogne : Rivadesella, Candas, Aviles, Villavieja (Rivadeo) ; EP. à San Vicente. S. - Gijon. Durieu. - La Corogne. Laz. - Santander, le Ferrol. CoL.

A. latifrons Kx. - Le Doel, Philippine (Escaut), Swin (Fl. occle). Kx.

B. spiralis Chauv. - Finistère. CRN.

C. vesiculosus Ag. - Avec le latifrons. Kx.

Forme naine et très réduite. - Pempoul, en un endroit que les plus hautes marées seulement atteignent. J. Сн. - Biarritz. Voyage de l'Unio. 


\section{Fucus platycarpus Thur.}

\section{$=F \cdot$ vesiculosus evesiculosus Crn. $-F$. vesiculosus Sherardii} Auct.

EP. à Ostende. De W. - Bath (Escaut). H. V. H. - Gris-Nez, Audresselles; Wimereux, la Rochette; Boulogne, Fécamp, Etretat. D. - Le Havre. Bernard. - Port-en-Bessin, Grandcamp. D. Arromanches, Granville. Herb. J. B. de Bruxelles. - Côtes du Calvados et du Finistère. Desmaz. - Z. S. Cherbourg. Le Jol. Roscoff. Sir. - Z. S. Finistère. CrN. - Le Croisic. Fu. - Biarritz, Guéthary, St. Jean de Luz. S. - Santander. Laz. - San Vicente, Gijon, Rivadeo; hermaphrodite et dioïque à la Corogne. S. -- Castro (Asturies). Durieu.

Toujours à la limite supérieure du flot, au-dessus du $F$. vesiculosus d'après THUR.

A. limitaneus ( $=F$. limitaneus Mont. $)-Z$. S. roches dans la baie du Toulinguet, côte E. (Crozon). J. Сн. - Biarritz, en avant du Port-Vieux et près de la villa Belza. S. - St. Jean de Luz, en face du Casino et sur les marches de la jetée Ste-Barbe. S. Villavieja et Figueras (Rivadeo). S.

De Toni rattache cette variété au $F$. vesiculosus. Elle mesure de 5 à 10 cent. de hauteur, assez robuste, et elle offre toutes les transitions entre les pieds les plus petits et la taille normale de l'espèce.

Une forme très réduite du Fucus limitaneus, exemplaires bien fructifiés d'un centimètre de hauteur et moins encore, en gazons serrés, a été signalée au cap Figuier (Fontarabie) par M. Sauvageau. Je l’y ai récoltée en 1903. En 1904, j’ai trouvé à Andagorria (Hendaye) d'énormes surfaces de roche, plus d'un hectare, couvertes par les gazons de ce Fucus nain, gazons compactes ou interrompus; tous les pieds sont exactement pareils, sans aucune transition avec des formes plus grandes; enfin vraiment l'allure d'une espèce. Je l'ai distribuée en séance de la Soc. Bot. de Belgique décembre 1904.

B. spiralis Rosenvinge. - San Vicente (Boria); Gijon (Jove). S.

Les principaux caractères qui distinguent le $F$. platycarpus du $F$. vesiculosus sont les suivants :

Station plus élevée que le $F$. vesiculosus.

Pas de vésicules aérifères. vésicules.

Carpomates marginés. . . . non marginés.

Hermaphrodite . . . . dioïque.

Cependant nous ferons observer:

J. Ag. a vu le F. vesiculosus hermaphrodite.

Sauvageau a vu le $F$. platycarpus à la Corogne avec conceptacles exclusivement $\sigma^{x}$

A Port-Arotcha, le $F$. platycarpus descend plus bas que le vesiculosus.

Le $F$. vesiculosus sans vésicules n'est pas rare.

Parmi les formes du $\boldsymbol{F}$. vesiculosus que mentionne Kickx, il s'en trouve trois (n $\left.{ }^{0 s} 18,19,21\right)$ avec carpomates marginés. Les n ${ }^{\text {os }} 12$ à 22 incl. n'ont pas d'aérocystes. 


\section{Fucus serratus L.}

Tout le littoral belge (EP.?) Kx. - EP. entre Ostende et Nieuport. Herb. W. - Z. M. tout le littoral de Dunkerque au Havre. D. - Z. M. et Z. I. Cherbourg. LE JoL. - Iles anglo-normandes. H. V. H. - Roscoff. Sir. - Le Croisic. D. FL. - Finistère. CrN. Sur la plage au cap Ferret (Arcachon). Chantelat. - Étang d'eau saumâtre à Capbreton. LAPEYrère. - San Vicente, Gijon. S. - Le Ferrol. Cos. - Santander, Comillas, Candas, la Corogne. Laz.

A. integer Turn. (=F. angustifolius With.) - San Vicente de la Barquera, Candas, la Corogne. Laz.

B. integerrimus Ag. - La Panne (EP. ?) Herb. Ricourt.

C. Iatifolius Turn. - La Panne (EP. ?) Herb. Ricourt.

D. macrodon West. - Ostende (EP. ?) Kx.

Frondes larges et tordues. - Havre de Peral. S.

De petite taille. - Rivadeo. S.

\section{F. spiralis L.}

$=F$. platycarpus Aresch. - F. Sherardii spiralis Aresch. F. vesiculosus spiralis Ag. - F. vesiculosus contortus $\mathrm{Kx}$.

Côtes de France. De Toni. - Arromanches, Cherbourg, îles Chausey. Herb. Zan. - Finistère. CRn.

\section{F. vesiculosus $\mathrm{L}$.}

Escaut à Anvers. J. CH. - Sur tous les murs et pilotis du littoral belge. Kx. De W. etc. - Z. S. et Z. M. plus bas que le F. platycarpus, sur tout le littoral de Dunkerque au Havre. D. Z. M. Cherbourg. Le Jou. - Iles anglo-normandes. H. V. H. Roscoff. Sir. - Finistère. CRN. - Le Croisic. FL. D. - Bords du bassin d'Arcachon. Chantelat. - Bayonne. Darracq. - Biarritz. Postels. - Guéthary, San Vicente, Gijon; Rivadeo? S. - Santander, Candas, le Ferrol, la Corogne. Laz. - Embouchure de la Bidassoa et de la rivière de Bilbao. CoL.

A. balticus J. Ag. - Z. S. Penfeld. Très petit, de 2.4 cent., baigné seulement par très haute mer, Kervallon. CRN.

B. crispatus. - San Vicente (Lin̉era). S.

C. cystocarpus Ktz. - Golfe de Gascogne. KTz. in CoL.

D. evesiculosus. - Guéthary, San Vicente, la Corogne. S.

E. laterifructus Grév. - Cherbourg, avec le type. LE JoL. 
F. linearis Ktz. (=F. ceranoides Esp. - F. distichus Lightf.) Golfe de Gascogne, côte espagnole ? CoL. Col.

G. monocystus Ktz. - Santander, côtes asturiennes, le Ferrol.

H. spiralis Turn. - Bords du bassin d'Arcachon. Chantelat. - Le Ferrol, la Corogne. Col.

I. volubilis. - Iles Chausey. LEN.

\section{Formes de la côte belge, d'après Kickx.}

1. propinquus. Incl. $F$. volubilis Huds.

2. macrocarpus $=F$. vesiculosus longifructus Bind.

3. acutus.

4. Westendorpii.

5. virgatus.

6. yolycarpus.

7. vulgaris ; 3 formes, dont une = Var. foliaceus Lam.

8. obtusus $=F$. divaricatus L. Spec.

9. monocystus $=F$. divaricatus L. Syst.

10. mempiscus.

11. alternans $=$ var : globosus Roth.

12. chondriformis.

13. balticus = Fucus balticus Ag. Décades. = Fucus subecostatus Ag. Syst.

14. lutarius $=F$. spiralis $=F$. axillaris spiralis $\mathrm{J}$. Ag.

15. biformis.

16. flabellatus $=F$. vesiculosus linearis $\mathbf{A g}$.

17. torquescens $=F$. spiralis Stackh (pro parte).

18. contortus $=F$. vesiculosus spiralis Ag. $=F$. spiralis $\mathrm{L}_{0}=F$. spiralis Stackh (pro parte).

19. spiralis.

20. longifructus $=F$. angustifolius With. $=F$. vesiculosus angustifolius Turn. $=F$. spiralis angustifolius Stackh.

21. inflatus $=F$. vesiculosus inflatus Roth.

22. Coemansii.

23. undulatus.

24. macrocystus.

25. laterifructus $=F$. disciplinalis Borrich.

26. phyllocarpus.

27. pachyphyllus.

\section{Ascophyllum nodosum (L.) Le Jol.}

= Ozothallia Dene. - Fucodium J. Ag. - Halidrys Lyngb.

Fixé et très abondant sur une longue jetée de pierre allant de la rive au marégraphe de Bath. (Escaut à 30 kil. en aval d'Anvers) ; Hansweert (50 kil. d'Anvers). H. V. H. - EP. sur tout le littoral belge. De W. - Dunkerque. Desmaz. - Gris-Nez, la Crèche 
(Boulogne); souvent EP. de Dunkerque au Havre. D. - Z. M. Cherbourg. Le Jou. - Iles anglo-normandes. H. V. H. - Ile Bréhat, forme géante. J. Сн. - Roscoff. Sir. - Z. S. et Z. M. Roscoff. J. Сн. - Finistère. Crn. - Le Croisic. Fu. D. - La Teste-de-Buch. Chantelat. - Biarritz. Postels, et voyage de l'Unio. - EP. Biarritz, Guéthary, St. Jean de Luz. S. - Rare en EP. à Guéthary. J. CH. Bilbao, Santander, le Ferrol. Col. - Chenal de Pasajes (fixé, abondant); rivières de San Vicente et de Rivadeo. S. - La Franca, Candas. Laz.

A. scorpioides (Hornem.) Rke. - EP. à Nieuport. Kx.

\section{Pelvetia canaliculata (L.) Dcne. et Thur.}

\section{= Fucodium J. Ag.}

Z. S. sur pilotis à l'entrée du chenal de Nieuport. Kx. - EP. à Ostende. W. - De $2 \mathrm{ctm}$. seulement de hauteur, bien fructifié, Dunkerque. Desmaz. - Gris-Nez, Wimereux, la Rochette, le Portel. D. - Dieppe. Desmaz. - Trouville. D. - Z. S. Hommet, Querqueville. LE Jou. - Iles anglo-normandes. H V. H. - Roscoff, Z. S. même dans les régions que mouillent seulement les plus hautes mers. J. CH. - Z. S. Finistère. Crn. - Le Croisic. Fu. D. - Biarritz. Postels. - De 2 à 3 ctm., Gijon et Castro. Durieu. - Santander, Candas, le Ferrol. Col. - San Vicente, Gijon, Rivadeo, la Corogne. S.

\section{Bifurcaria tuberculata Stackh.}

\section{= Pycnophycus Ktz. - Fucodium J. Ag.}

EP. littoral belge. Kx. - Z. I. Cherbourg, Granville. Chauv. Iles anglo-normandes. H. V. H. - Roscoff. Sir. - Z. I. Finistère. Crn. - Lorient. Herb. Zan. - Belle-Isle. Herb. J. B. de Bruxelles. - Le Croisic. Fu. - San Vicente, Gijon, Rivadeo, la Corogne. S. De $5 \mathrm{ctm}$. de hauteur, bien fructifié, Gijon. Durieu. - Santander, Aviles, Artedo (Asturies). CoL.

De même que le Laminaria saccharina, cette espèce donne en séchant une efflorescence de glycose, produite aux dépens des géloses de la cellule.

\section{Himanthalia lorea (L.) Lyngb.}

EP. sur tout le littoral belge. Kx. - EP. sur toute la côte de Dunkerque au Havre. D. - Côtes du Calvados. Chauv. - Z. I. Cherbourg. Chauv. - Iles anglo-normandes. H. V. H. - Roscoff, Z. I. vastes surfaces uniquement occupées par cette espèce. J. CH. 
- Z. I. Finistère. CRN. - Le Croisic. FL. D. - La Teste-de-Buch. Chantelat. - Guéthary, un seul pied fixé; EP. abondante à Biarritz, Guéthary, St. Jean de Luz. S. - EP. abondante à Guéthary certains jours, et puis pendant des semaines, plus un seul pied. J. Сн. - En place, San Vicente, Gijon, Rivadeo, la Corogne. S. - Santander, Comillas. LAZ. - Le Ferrol. CoL.

A. gracilis. - Cherbourg. HeRB. LeN.

* Rechercher la forme stérile à lanières cylindriques.

\section{Halidrys siliquosa (L.) Lyngb.}

$=$ Cystoseira Ag.

EP. littoral belge. DE W. - EP. à Nieuport. W. - Wimereux, Petites-Dalles, Fécamp, Yport, Etretat. D. - Bruneval, Antifer. Bernard. - Quihot, Langrune, Port-en-Bessin, Grandcamp. D. Z. I. Cherbourg. LE Jol. - Iles anglo-normandes. H. V. H. St. Malo. Melle Lebrun. - Roseoff. Sir. - Z. I. Finistère. Crn. Le Croisic. Fu. D. - Ile de Ré, limite S. de la plante fixée. S. La Teste-de-Buch. Chantelat. - Biarritz. Postels. - EP. Biarritz, Guéthary, St. Jean de Luz. S. - Gijon. Durieu. - Santander, Comillas, San Vicente, la Franca, Candas, Llanes. Laz.

A. denudata Lyngb. - La Panne. VAn de Vyvere. - Chenal de la rade de Brest, EP. CrN.

B. minor Grev. - Nieuport (EP.?) Kx.

Forme courte et compacte. - Arromanches. Herb. J. B. de Bruxelles.

Se désarticule en septembre, et le flot emporte et dissémine les tout petits rameaux fructifères.

Cystoseira Abies-marina (Gmel.) Ag.

$=$ C. Montagnei J. Ag.

Biarritz. Voyage de l'Unıo. EP. ? - Sur Araignée de mer à Guéthary. S.

Espèce de la Méditerranée et de l'Atlantique méridionale.

\section{C. abrotanifolia Ag.}

Portbail (Manche); Cherbourg. Herb. J. B. de Bruxelles. Golfe de Gascogne. Bons. - San Vicente de la Barquera. Laz. Santander et provinces basques. CoL. 


\section{C. barbata (Good, et Woodw.) Ag.}

$=$ C. Hoppii Ag. - C. granulata Della Chiaje.

EP. entre Nieuport et la Panne. Kx. - Aurigny. Mme Gaudion. - Guernesey. Melle Lelièvre. - Brest. Len. - Z. M. côte S. du Finistère. CRN. - San Vicente de la Barquera, la Franca, la Corogne. Laz. - Le Ferrol. Col.

\section{Gystoseira concatenata (L.) Ag.}

\section{$=$ C. granulata concatenata Menegh.}

EP. à Ostende. Landszweert, in Herb. J. B. de Bruxelles. Cherbourg. Herb. J. B. de Bruxelles. - EP. à Guéthary. S.

Espèce de la Méditerranée et de l'Atlantique méridionale.

\section{G. discors (L.) Ag.}

$=$ C. foniculacea Grev. - C. paniculata Ktz.

EP. Bruneval. Bernard. - Z. M. Cherbourg. Le Jol. - Iles anglo-normandes. H. V. H. - St. Malo. HerB. J. B. de Bruxelles. Roscoff. J. CH. - Z. I. dragué, rade de Brest. Crn. - Le Croisic, flaques élevées. FL. D. - Biarritz, Guéthary, St. Jean de Luz. S. - Santander. Col. - San Vicente, Gijon, Rivadeo, la Corogne. S. - Le Ferrol. CoL.

\section{G. ericoides (L.) Ag.}

Dieppe, St. Valéry-en-Caux, Fécamp. Leturquier, - Bénouville. Bernard. - Port-en-Bessin, Grandcamp. D. - Z. I. Cherbourg. Le Jol. - Iles anglo-normandes. H. V. H. - St. Malo. Herb. J. B. de Bruxelles. - Roscoff. Sir. - Z. I. Finistère. Crn. - Lorient. Herb. Zan. - Flaques élevées, le Croisic. Fl. D. - La Teste-deBuch. Chantelat. - Biarritz, Guéthary, St. Jean de Luz, San Vicente, Gijon, Rivadeo, la Corogne. S. - St. Sébastien, Santander, Candas, le Ferrol. CoL.

\section{C. fibrosa (Huds.) Ag.}

$=C$. discors linearis Ag.

EP. à Ostende. Kx. - EP. entre Ostende et Blankenberghe. W. - EP. sur toute la côte de Dunkerque au Havre. D. - Dieppe. Leturquier. - Le Havre. Dubec. - Z. I. Hommet, Querqueville. Le Jol. - Iles anglo-normandes. H. V. H. - Carteret. Herb. Zan. - Granville. Chauv. - Roscoff. Sir. - Z. I. Finistère. Cri. - 
EP. sur la grande côte (le Croisic). D. - EP. la Teste-de-Buch. Chantelat. - Z. M. et Z. I. Biarritz, Gueethary, St. Jean de Luz, San Vicente, Gijon; EP. à Rivadeo; la Corogne. S. - Santander, Candas, la Franca. Laz. - St. Sébastien, Aviles. Col.

A. thesiophylla Len. - La Teste-de-Buch. Chantelat. Bayonne. Herb. J. B. de Bruxelles. - Biarritz. De Martens.

Gystoseira granulata (L.) Ag.

EP. Bruneval. Bernard. - EP. le Havre. Dubec. - Luc. Chauv. - Langrune. D. - Côtes du Calvados. Lev. - Z. M. Cherbourg. LE JoL. - Iles anglo-normandes. H. V. H. - Portbail (Manche). Herb. J. B. de Bruxelles. - Roscoff. Sir. - Z. I. Finistère. CRN. Chenal du Croisic. Fu. D.

A. Chauviniana Ktz. - Cherbourg. Herb. J. B. de Bruxelles.

$$
\text { C. humilis Schousb. }
$$

Semble une variété naine du $C$. discors (Bornet).

Tanger. Schousboe. - C'est peut-être la même qu'on trouve à Biarritz. BoRN.

\section{Sargassum bacciferum (Turn.) Ag.}

EP. à Ostende. Kx. - EP. à Dieppe. Leturquier. - EP. (une fois) Cherbourg. LE Jol. - EP. à Jersey. BRÉB. - Santander. CoL.

\section{S. linifolium Ag.}

Le Ferrol. CoL.

\section{S. vulgare Ag.}

Guipuzcoa, le Ferrol, la Corogne. CoL.

A. flavifolium Ktz. - EP. à Guéthary. S. J. Сн. - En septembre, abondant de Guéthary à Biarritz, EP. désarticulé en tout petits rameaux fructifères. J. Сн. - La Corogne. LAZ. 


\section{ORDRE DES DICTYOTÉES}

Dictyota.

Taonia.

Padina.
Haliseris.

Spatoglossum.

\section{Dictyota dichotoma (Huds.) Lamour.}

$=$ Zonaria Ag. $-D$. vulgaris $\mathrm{Ktz} .-D$. volubilis $\mathrm{Ktz} .-D$. spiralis Mont. - D. acuta Ktz.

EP. entre Nieuport et la Panne. Kx. - Tout le littoral, du Tréport à Grandcamp. D. - Z. M. et Z. I. Cherbourg. LE JoL. EP. Luc, Cherbourg, Granville. Chauv. - Iles anglo-normandes. H. V. H. - Roscoff. Sir. - Z. I. Finistère. CrN, - Le Croisic. FL. D. - La Teste-de-Buch, cap Ferret. Chantelat. - Biarritz, Guéthary, St. Jean de Luz, San Vicente, Gijon, Rivadeo, la Corogne. S. La Franca. Laz.

A. acuta Chauv. (=D. dichotoma rigida Crn. $)$ - EP. littoral belge. W. - Arromanches. Desmaz. - Portbail (Manche). Herb. J. B. de Bruxelles. - Bertheaume. Crn. - La Teste-de-Buch. Chantelat.

B. implexa J. Ag. (=D. intricata Chantelat). - Tout le littoral, de St. Valéry-en-Caux à Grandcamp. D. - Z. M. Cherbourg. Le JoL. - Portbail, Granville. Herb. J. B. de Bruxelles. - Iles anglo-normandes. H. V. H. - St. Malo. LEN. - Roscoff. Sir. - Variété la plus commune autour de Roscoff. J. Cн. - Z. I. le Délec, Ste-Anne, banc du Moulin. CrN. - Le Croisic. FL. D. - Cap Ferret (Teste-deBuch). Chantelat. - St. Sébastien. Col.

Cette variété arrive à la forme exactement capillaire : Roscoff et environs. J. Сн. Elle forme des gazons sur roche, épais et feutrés: Biarritz, Guéthary. J. Сн.

C. latifrons Holm. et Batt. - St. Vaast-la-Hougue, Arromanches. Herb. J. B. de Bruxelles. - Iles anglo-normandes. H. V. H. Roscoff. J. CH.

D. spiralis Mont. - Côtes du Calvados. Len. - Roscoff. J. Сн. - Anse du Minou. CrN. 


\section{Dictyota fasciola (Roth.) Lamour.}

\section{= Zonaria Ag.}

Sur les pierres (??), et sur les stipes de Laminaria rejetés entre Nieuport et la Panne. Kx. - Boulogne. Desmaz. in Herb. J. B. de Bruxelles. - La Corogne. CoL.

\section{D. ligulata Ktz.}

$=D$. fasciola ligulata Vin. $-D$. dichotoma elongata Crn. ?

Z. I. le Minou. Crs. - Arotcha, près de Guéthary ; Biarritz, St. Jean de Luz, San Vicente. S. - Abondante au printemps à Arotcha; très rare à la fin de l'été et alors de petite taille et en train de repousser. J. Сн.

\section{D. linearis (Ag.) Grev.}

$=D$. angustissima Ktz. $-D$. divaricata Lamour.

San Vicente de la Barquera, la Franca, Candas. Laz. - La Corogne. CoL.

Taonia atomaria (Good. \& Wood.) J. Ag. liata Lamour.

= Zonaria Ag. - Dictyota Grev. - Dictyota zonata et D. ci-

EP. à Ostende. Herb. J. B. de Bruxelles. - EP. la Panne. W. - La Roche-Bernard (Boulogne), et tout le littoral de Mesnilval à Grandcamp. D. - Luc. LeN. - St. Malo, St. Vaast, Arromanches. Herb. Zan. - Z. I. Flamands, Nacqueville. Le Jol. - Iles anglonormandes. H. V. H. - Z. I. Roscoff, en face de Perkiridic. J. CH. Z. I. le Minou, Bertheaume. CrN. - Morbihan. Herb. J. B. de Bruxelles. - Le Croisic. Fu. D. - La Teste-de-Buch, cap Ferret. Chantelat. - Biarritz, Guéthary, St. Jean de Luz, San Vicente, Gijon, Rivadeo. S.

A. ciliata Ag. - Luc, Arromanches, Port-en-Bessin. Chauv. Rivière d'Ares (la Corogne). CoL.

\section{Padina Pavonia (L.) Lamour.}

Z. I. sur pilotis, Ostende et Nieuport. Kx. - EP. à Ostende. W. - Dunkerque. Lestiboudois. - Souvent EP. Wimereux. Giard, Moniez. - St. Jouin. Bernard. - Quihot, Langrune, Port-en-Bessin, Grandcamp. D. - St. Vaast, Barfleur, Granville. Herв. J. B. de 
Bruxelles. - Z. M. Cherbourg. LE JoL. - Iles anglo-normandes. H. V. H. - Plage de Portez (Conquet). Langeron. - Le Minou, Bertheaume, Douarnenez. CRN. - Camaret. J. СH. - Belle-Isle. Herb. J. B. de Bruxelles. - Morbihan. Prounet. - Port et jetée du Croisic, grande côte. D. - Flaques élevées, le Croisic. FL. Chapelle d'Arcachon, Moullo. Chantelat. - Biarritz, Guéthary, St. Jean de Luz, San Vicente, Gijon, Rivadeo, la Corogne. S.

\section{Haliseris polypodioides (Desf.) Ag.}

= Dictyopteris Lamour. - Dictyopteris elongata Lamour.

Fécamp, Luc, Grandcamp. D. - Port-en-Bessin. Chauv. - Z. I. Hommet, Ste-Anne, Querqueville, Flamands. Le JoL. - Iles anglonormandes. H. V. H. - Carteret. Herb. J. B. de Bruxelles. - Granville. Chauv. - Roscoff. Sir. - Le Conquet. Langeron. - Z. I. Bertheaume, le Délec. CrN. - Camaret. J. Ch. - Lorient. Herb. Zan. - Belle-Isle. Lloyd. - Le Croisic. Fu. - Jetée du Croisic. D. Biarritz, Guéthary, St. Jean de Luz, San Vicente, Gijon, Rivadeo, Z. I. Plante mâle pêchée au large de Guéthary. S. - Santander, la Corogne. Laz. - Concha de Artedo (Asturies). Col.

Dégage à l'état frais une odeur forte et désagréable. Quatre pieds différents : mâles, femelles, avec tétraspores et stériles.

\section{Spatoglossum Solierii (Chauv.) Ktz.}

\section{$=$ Taonia ? J. Ag. - Dictyota Chauv.}

Z. I. anse du Minou, entrée du port de Brest. CRN. - Sur Araignée de mer à Guéthary. S.

De Toni indique: Côtes de France, d'après Solier, J. Agardh, Chauvin, Crouan, Bornet et Hohenacker. Mais il y a doute dans le texte; côtes de la Méditerranée ou de l'Atlantique ? - La station de Brest en douteuse. 


\section{ORDRE DES RHODOPHYCÉES OU FLORIDÉES}

\section{PORPHYRÉES ou BANGIALES}

Goniotrichum.

Neevia.

Erythropeltis.

Erythrotrichia.
Bangia.

Porphyra.

Wildemania.

^ Goniotrichum cervicornu Reinsch.

Découvert à Plymouth en 1897 par A. CHURCH.

Le genre Goniotrichum Ktz. doit peut-être se rapporter aux Palmellacées ou aux Nostochinées: ThUR. A la suite des Bangiacées, dans les genres douteux: Schmтz. Dans les Bangiacées: Debray.

$\star$ Neevia repens Batt.

Trouvé à Deal, comté de Kent. BATT.

* Erythropeltis discigera (Berth.) Schm.

$=$ Erythrotrichia Berth.

Espèce découverte par Berthold sur Cystoseira dans le golfe de Naples.

A. Flustræe. - Comté de Kent. Batt.

Erythrotrichia ceramicola (Lyngb.) Aresch.

$=$ Callithamnion simplex Crn. - Goniotrichum Ktz.

Z. S. Gris-Nez. D. - Arromanches. Chauv. - Cherbourg. LE JoL. - Iles anglo-normandes. H. V. H. - Dragué, rade de Brest; Z. M. Argenton. CRN. - Biarritz, Guéthary, St. Jean de Luz, San Vicente, Rivadeo. S.

A. vertebrata Crn. - Dragué, rade de Brest. CRN. 


\section{Erythrotrichia investiens (Zan.) Born.}

\section{= Bangia Zan. - Bangia tenuissima Ktz.}

Sur Cladophora utriculosa, Z. I. pointe S. de l'île Callot. Dét. Heydrich. J. CH. - San Vicente. S.

\section{E. reflexa (Crn.) Thur.}

$=$ Bangia Crn. Hauck. - Porphyra Crn.

Mles anglo-normandes. Jersey 1903. H. V. H. - Sur Fucus balticus, Kervallon, la Villeneuve. CRN. - Le Croisic. FL. - San Vicente. S.

\section{E. Welwitschii Batt.}

Iles anglo-normandes. H. V. H.

\section{Bangia ciliaris Carm.}

= Erythrotrichia Thur. - Porphyra bangiiformis Ktz.

EP. à Ostende, sur Céramiées. W. - Arromanches, sur Laurencia. Chauv. - Sur Laurencia, Z. S. Hommet, Querqueville, Nacqueville. Le Jol. - Batterie du Fer-à-cheval, Ste-Anne. CrN. Golfe de Gascogne sur Chondrus crispus. CoL.

A. crispula Chauv. - Arromanches. Len.

B. elegans Chauv.

= Goniotrichum Zan.

EP. à Ostende. W. - Arromanches. Chauv. - Côtes du Calvados. LEN. - Rochers de Longlet. THUR. - Sur Cladophora rupestris, Duon, Roscoff. J. CH. - Kervallon, et dragué en rade de Brest. CRN. - Le Croisic. Fu. - Biarritz, Guéthary, St. Jean de Luz, San Vicente, Rivadeo, Gijon. S.

\section{B. fusco-purpurea (Dillw.) Lyngb.}

$=B$. tristis De Not. - B. arctica Fosl. - De Toni la donne comme sous-espèce de B. atro-purpurea (Roth.) Ag.

EP. à Philippine. Kx. - Wimereux. De Block. - Z. S. Antifer, Bruneval. Bernard. - Le Havre, murailles et pilotis. Duboc. Dupray. - Calvados. Chauv. - Z. S. Hommet, Querqueville, Digue; bois submergés à l'entrée du port militaire de Cherbourg. LE JoL. Iles anglo-normandes. H. V. H. - Z. S. Trégana, Bertheaume. CRN. - Villa Belza et près du phare à Biarritz. S. - Le Ferrol. Col. 


\section{Bangia Le Jolisii De Not.}

\section{$=B$. fusco-purpurea Desmaz.}

Z. S. à l'entrée du port militaire. LE JoL. - Rade de Brest. CRN.

\section{B. versicolor Ktz.}

$=$ B. subaequalis $\mathrm{Ktz} .-B$. atro-purpurea Aresch.

Z. S. Kervallon. CRN.

\section{Porphyra atro-purpurea (Olivi.) De Toni.}

$=P$. leucosticta Thur. - P. laciniata Crn. - P. vulgaris Lloyd. - P. livida De Not. - P. vermicellifera Ktz.

Z. I. Yport, Antifer. Bernard, - Z. I. Cherbourg (atteint $20 \mathrm{ctm}$ ). LE JoL. - Granville. BRÉB. - Iles anglo-normandes. H. V. H. La Corogne. Laz. - Roscoff. Melles V. et K. - Port-Vieux de Biarritz (longueur $20 \mathrm{ctm}$ ). S.

D'après Debray, le Porphyra linearis Grev. s'élargit avec la croissance $[P$. vulgaris $\mathrm{Harv}$.] et devient finalement $P$. laciniata Ag. Thuret n'admet dans l'Océan que 2 espèces, savoir : $P$. laciniata Ag. et $P$. leucosticta Thur.

P. coccinea J. Ag.

$=P$. minima $\mathrm{Crn} .-P$. delicatula Welw.

Dragué en rade de Brest. CRN.

Wildemania amethystea (Ktz.) De Toni.

= Porphyra Ktz.

Côtes de France et d'Angleterre. Shutrleworth.

W. laciniata (Lightf.) De Toni.

$=$ Porphyra Ag. - Porphyra linearis Grev. - Ulva umbilicalis Lightf. - Porphyra laciniata umbilicalis Ag.

Filets des pêcheurs à Blankenberghe. Kx. - EP. à Ostende. W. - Dunkerque, Blanc-Nez, du Gris-Nez au Portel, Cayeux, Ault, Mers, et tout le littoral de la Seine inf. et du Calvados. D. - Le Havre. Martens. - Z. S. Cherbourg. Le Jol. - Iles anglo-normandes. H. V. H. - Roscoff. Sir. - Z. I. Finistère. CrN. - Le Croisic. Fu. D. - San Vicente, Rivadeo, la Corogne. S. - Le Ferrol. Col.

A. linearis. - Cherbourg. LE JoL. - Iles anglo-normandes. H. V. H. - Brest, le Croisic (diminuée jusque 2 ou $3 \mathrm{ctm}$ ). Desmaz. - Port-Vieux de Biarritz, la Goureppe (Guéthary). S. 
B. umbilicalis. - Jersey. BATt. - Ile de Bas. Melles V. et K. - Le Croisic. FL.

Wildemania linearis (Grev.) De Toni.

= Porphyra Grev. Ktz. J. Ag. - Porphyra vulgaris Harv. pro parte.

Banc d'huîtres au large de la Panne. Kx. - Z. S. Cherbourg. LE JoL. - Mes anglo-normandes. H. V. H. - Z. S. sous le château de Brest, Laninon. CrN.

W. umbilicalis (L.) De Toni.

= Porphyra Ktz. - Ulva purpurea Roth. - Porphyra vulgaris et $P$. purpurea Ag.

Pilotis des écluses de Heyst, estacade de Nieuport. Kx. Calvados. Len. - Z. S. Cherbourg. Le Jol. - Aurigny. Mme Gaudion. - Jersey. Melle Whrte. - Roscoff. Sir. - Finistère. Crn. - Sur Moules au Croisic. Lloyd. - Côtes des Asturies et de Galicie, sud du golfe de Gascogne. CoL.

A. divisa (= Ulva umbilicalis lanceolata Lyngb.)

B. simplex (= Porphyra linearis Auct. non Grev.)

\section{HELMINTHOCLADIÉES}

Colaconema.

- Acrochætium.

Nemalion.

Helminthocladia .
Helminthora.

Chantransia.

Liagora.

\section{* Colaconema Bonnemaisoniæ Batt.}

Parasite sur Bonnemaisonia asparagoides. Signalé à Plymouth et Berwick. BatT.

C. reticulatum Batt.

Parasite sur Desmarestia Dudresnayi. Jersey 1903. H. V. H. 


\section{Acrochætium Ghylocladiæ Batt.}

= Chantransia Batt. - Colaconema Batt.

Dans le thalle de Chylocladia ovalis. Jersey 1903. H. V. H.

* A. pulchrum Batt. - Dans les Sertulaires. A rechercher.

$\star$ A. efflorescens Näg.

$=$ Callithamnion J. Ag. - Chantransia Kjellm.

Sur Dudresnaya coccinea et Naccaria Wigghii. Signalé à Swanage. BATT.

\section{A. endozoicum Batt.}

= Chantransia Darbyshire.

Dans le corps gélatineux de Flustra hispida. Dét. Heydrich. Roche du Loup, Roscoff ; au pied de la falaise de Pen-Tir (Crozon). J. CH.

A. entophyticum Batt.

Dans le thalle de Dasya coccinea. Signalé à Plymouth. Batr.

Nemalion lubricum Duby.

= Mesogloia Bertolonii Moris. - Helminthora Nemalion Ktz. - Chordaria Nemalion Ag.

Z. I. Wimereux, Pointe-aux-Oies, sur bancs de Moules. De Block. - Iles anglo-normandes. H. V. H. - Carantec. J. Сн. - Roscoff. Sir. - Batterie du diable, le Minou, Bertheaume. Crn. - Pied de la falaise de Toulinguet (Crozon). J. CH. - Z. S. le Croisic. FL. - La Pierre-plate (Croisic) D. - Biarritz, Guéthary, St. Jean de Luz, San Vicente. S. - La Corogne. Laz.

A. dichotomum Crn. - Anse du Minou. CrN.

N. multifidum (W. et M.) J. Ag.

$=$ Mesogloia Ag. - Chordaria Lyngb. - Helminthora Ktz.

Z. I. Wimereux, Pointe-aux-Oies, sur banes de Moules. De Block. - Z. M. Hommet, Querqueville, Nacqueville, île Pelée. LE JoL. Iles anglo-normandes. H. V. H. - Z. M. rade de Brest, Laninon. Crn. - Pied de la falaise de Toulinguet (Crozon). J. Сн. - Morbihan. Herb. J. B. de Bruxelles. - Biarritz, Guéthary, St. Jean de Luz, San Vicente, Ria a los Rosales. S.

Les espèces $N$. lubricum et multifidum sont à peine à séparer, je crois. C'est seulement la ramification qui fait la différence, et celle-là est un peu variable chez les deux espèces; ainsi il est souvent difficile de les distinguer avec sûreté. REINBOLD. 
Helminthocladia Hudsonii (Ag.) J. Ag.

= Mesogloia Ag. - Liagora viscida Schousb.

Z. I. pointe de Corson. CrN.

H. purpurea (Harv.) J. Ag.

= Mesogloia Harv. - Nemalion Chauv. - Dumontia Calvadosii Lamour.

Fécamp, Bénouville, Luc. D. - Z. I. île Pelée. Bons. - Iles anglo-normandes. H. V. H. - Roscoff. Sir. - Z. I. chenal de l'île Verte. J. Сн. - Z. I. pointe de Corson, Porspoder, Argenton. CrN. - Pied de la falaise de Pen-Tir (Crozon). J. Сн. - Camaret. J. Сн. - Belle-Isle. Lloyd. - Morbihan. Prouhet. - Le Croisic. Fl. Z. I. Guéthary, St. Jean de Luz, San Vicente, Gijon. S.

\section{Helminthora divaricata (Ag.) J. Ag.}

= Mesogloia Ag. - Nemalion Ktz. - Dudresnaya J. Ag. Nemalion clavatum Ktz.

St. Vaast. Len. - Z. I. Cherbourg. LE Jol. - Iles anglonormandes. H. V. H. - Roscoff. Sir. - Ste-Anne, le Délec, Bertheaume. CRN. - Belle-Isle. Desuaz.

\section{Chantransia corymbifera Thur.}

= Callithamnion caespitosum J. Ag. - Call. Codii Crn. - Ch. caespitosa Batt. - Acrochaetium Batt.

Gatteville et Tatihou. MALARD et KuckUcK. - Sur Ceramium rubrum, Z. I. Querqueville. Thur. - Roscoff. Sir. - Batterie du Diable, anse du Minou, sur Patella et Codium CRN. - Guéthary. Lespinasse. Thur.

Gh. Daviesii (Dillw.) Thur.

= Callithamnion Lyngb. - Call. luxurians Desmaz.

EP. à Nieuport. W. - Wimereux, Pointe-aux-Oies ; PetitesDalles, Fécamp, Etretat, St. Jouin, Grandcamp. D. - Côtes du Calvados. Len. - Z. I. Cherbourg, Granville. Chauv. - Iles anglonormandes. H. V. H. - Dragué sur Dudresnaya coccinea à Duon (Roscoff.) J. CH. - Sur divers Ceramium, au Conquet. Langeron. Sur Cystoseira discors, au Croisic. Thur. - Biarritz, Guéthary, St. Jean de Luz, Gijon. S. 
* Chantransia irregularis Reinscl.

Sur Polysiphonia byssoides. Signalée à Weymouth. DE TonI.

\section{Ch. microscopica (Näg.) Fosl}

= Callithamnion J. Ag. - Acrochaetium Näg.

Jersey, sur Calliblepharis ciliata, 1902. H. V. H. - Roche du Loup (Roscoff) sur Bryozoaire. J. Сн. - Anse du Minou, Z. I. sur Enteromorpha compressa. CRN. - Sur Chondrus, la Goureppe. Dét. Heydrich. J. Сн.

Ch. mirabilis (Suhr.) Heydr. Näg.

$=$ Trentepohlia Suhr. - Callithamnion Ktz. - Acrochaetium

Sur Chondrus et Furcellaria. Littoral français de l'Atlantique. SuHR. - Dans Epicrisis d'Agardh : espèce parasite, côtes de France. - Sur Corallina rubens, Guéthary. Dét. Heydrich. J. Cн.

Ch. secundata (Lyngb.) Thur.

= Callithamnion J. Ag. - Call. Daviesii secundatum Lyngb. - Call. Lenormandii Suhr. - Call. microscopicum Näg.

Gris-Nez; Wimereux, Pointe-aux-Oies et Croy ; porte de la retenue de Fécamp. D. - Bénouville, Bruneval. Bernard. - Arroman. ches. Herb. Zan. - Cherbourg. Le Jol. - Iles anglo-normandes. H. V. H. - Sur Cladophora utriculosa, Z. M. pointe N de l'ŝle Callot. J. Сн. - Finistère. CRN. - Biarritz, Guéthary, St. Jean de Luz. S. - Sur Cystoseira, Guéthary, Andagorria. J. CH. - San Vicente de la Barquera. Laz.

\section{Ch. trifila Buffh.}

Sur Cladophora utriculosa, pointe N. de l'île Callot. Dét. Heydrich. J. Cн.

Gh. virgatula (Harv.) Thur.

= Callithamnion Harv. - Call. Daviesii J. Ag.

Sur Enteromorpha- compressa, Ostende. W. - Wimereux. D. - Sur Rhodymenia palmata, Arromanches. HerB. J. B. de Bruxelles. - Hommet, Querqueville. Le Jol. - Iles anglo-normandes. H. V. H. - Roscoff, sur Ceramium rubrum. J. CH. - Anse du 
Délec, banc du Château, Laninon, anse du Minou. CRN. - Toulinguet (Crozon). Dét. Reinbold. J. CH. - Sur Zostères, le Croisic. FL.

A. luxurians (= Ch. luxurians J. Ag. Näg.) - Iles anglo-normandes. H. V. H.

B. minutissima $(=C h$. minutissima Suhr. Ktz.) - Iles anglonormandes. H. V. H.

Liagora viscida (Forsk.) Ag.

Z. M. baie de Bertheaume, île de Sein. CRs. - Guéthary, Arotcha. S. - Abondante, Guéthary, Z. I. en juillet, au large à gauche de la plage des bains; presque disparu en septembre. Ј. Сн. - Gijon. S.

\section{CHÉTANGIÉES}

Scinaia.

Choreocolax.

Scinaia furcellata (Turn.) Biv.

= Ginnania Mont. - Halymenia Ag.

Audresselles, Wimereux, le Portel, Petites-Dalles, Fécamp, Yport, Etretat. D. - St. Jouin. Bernard. - Luc, Port-en-Bessin. D. - Côtes du Calvados (de 2 à $10 \mathrm{ctm}$ ). Len. - Arromanches. Herb. $\mathrm{Z}_{\mathrm{AN}}$. - St. Vaast. Le JoL. - Iles anglo-normandes. H. V. H. Roscoff. Sir. - Dragué sur coquilles à Astan. J. CH. - Abondant et bien fructifié, plages d'Ouessant. Langeron. - Z. I. Laninon, SteAnne, le Délec. Crn. - Le Croisic. Fl. D. - Biarritz, Guéthary, St. Jean de Luz, San Vicente, Gijon, Rivadeo, la Corogne. S.

A. subcostata J. Ag. (= Halymenia J. Ag. - Ginnania pulvinata subcostata Ktz.) - Laber-Ildut, EP. CRN. - EP. à Rivadeo. S.

\section{Choreocolax Polysiphoniæ Reinsch.}

Guernesey, Juin 1902. Mme Hamber. - Sur Polysiphonia fastigiata, Roscoff. J. Сн. - Au pied de la jetée du phare de Camaret. J. CH.

$\star$ Ch. tumidus. Reinsch.

Sur Ceramium rubrum, Cystoclonium purpurascens etc. A rechercher. 


\section{GÉLIDIACÉES}

Harveyella.

Atractophora.

Naccaria.
Pterocladia.

Gelidium.

Caulacanthus.

Harveyella mirabilis (Reinsch.) Schm. et Rke.

= Choreocolax Reinsch.

Sur Rhodomela subfusca, Tatihou. MALARD. - Cherbourg. Born. - Jersey 1902 (actuellement limite S. de l'espèce). H. V. H.

H. pachyderma Batt.

= Choreocolax Reinsch. - Choreocolax albus Kuck.

Sur Gracilaria confervoides, Tatihou. Malakd et Kuckuck. Limite S. actuelle.

\section{Atractophora hypnoides Crn.}

\section{= Naccaria J. Ag.}

Iles anglo-normandes. H. V. H. - St. Malo. Thur. - St. Pol de Léon, EP. Dudresnay. - Rade de Brest, dragué ; St. Mare, EP. CRN. - Guéthary. Thur.

\section{Naccaria Wigghii ('Turn.) Endl.}

= Hypnea Lamour. - N. Vidovichii Menegh.

Luc. Chauv. - St. Vaast. Herb. J. B. de Bruxelles. - Z. M. jetée E. du port des Flamands à Cherbourg (un seul pied). Bons. Carteret. Herb. ZAN. - Iles anglo-normandes. H. V. H. - Roscoff (sans autre indication). Melles V. et K. - Rade de Brest, dragué; Bertheaume, Lannevez, EP. et Z.I. (ne découvrant pas). CRN. Le Croisic. D. - Guéthary. Thur. Lespinasse.

Pterocladia capillacea (Gmel.) Born. et Thur.

= Gelidium Ktz. - Gelidium corneum pinnatum Grev.

Petites-Dalles, Fécamp, Yport, Bénouville, Etretat, Antifer, Bruneval, St. Jouin, Port-en-Bessin, Grandcamp. D. - Iles anglonormandes. H. V. H. - Anse du Minou, Corson. CrN. - Biarritz, 
Guéthary, St. Jean de Luz, San Vicente, Gijon, Rivadeo, la Corogue. S. - Santander, Candas. Laz. - Très réduit sur Patelle, Port-Vieux (Biarritz). J. Сн.

Bien variable. Offre des transitions à Gelidium sesquipedale Thur. et à Gelidium attenuatum Thur. REINBOLD.

\section{Gelidium attenuatum Thur.}

$=$ G. corneum attenuatum Hook.

St. Jean de Luz, San Vicente, Gijon, la Corogne. S.

G. cartilagineum (L.) Gaill.

= Sphaerococcus Ag. - G. rigidum Ktz. - G. concatenatum Lamour.

EP. côtes belges. W. - San Vicente de la Barquera, Candas. LAz. - Santander. CoL.

\section{G. corneum Lamour.}

Iles anglo-normandes. BATt. - Granville (de 2 à $10 \mathrm{ctm}$ ). LEN. - Roscoff. Sir. - Le Conquet (diff. formes). Langeron. - Le Croisic. Fl. D. - Cap Ferret (Teste-de-Buch). Chantelat - Bayonne. AGardh. - - Biarritz, Guéthary, St. Jean de Luz. S. - Santander, Candas, la Corogne Cos.

A. capillaceum Mont. Très variable lui-même; parfois réduit à 2 ou $3 \mathrm{ctm}$. et capillaire. - Cherbourg, Bayonne. Herb. J. B. de Bruxelles. - Santander. Col. - Biarritz, Guéthary. J. Cн.

B. clavatum Grev. - Arromanches. HerB. J. B. de Bruxelles. Voir G. pusillum.

C. claviferum Harv. - Z. I. Cherbourg. LE JoL. - Roscoff. Sir. - Anse du Minou, pointe de Corson. CrN. - Z. I. Belle-Isle. Luoyd. - Morbihan. LeurèvRe.

D. gracillimum Bréb. - Piron (Manche). BrÉB.

E. luxurians Crn. - Roscoff. Sir. - A l'entrée du port militaire de Brest. CRN.

F. sericeum Ag. - Port en-Bessin, Cherbourg, Granville. Chauv. - Biarritz. Endress. - La Corogne. Col.

G. tricuspidatum Chauv. - Port-en-Bessin. Chauv. 


\section{Gelidium crinale (Turn.) Lamour.} nalis Ag.

$=$ G. corneum crinale J. Ag. - Sphaerococcus corneus cri-

Gris-Nez; Wimereux, Croy ; Mesnilval, Dieppe, St. Valéry-enCaux, Petites-Dalles, Fécamp, Bénouville, Bruneval, Arromanches, Port-en-Bessin. D. - St. Vaast, Cherbourg. Herb. J. B. de Bruxelles. - Iles anglo-normandes. H. V. H. - St. Marc, Moulin-Blanc. CRN. - Le Croisic. Fl. - Plage d'Arcachon, EP. Chantelat. - Biarritz, Guéthary, St. Jean de Luz, San Vicente, Gijon. S. - Le Ferrol. CoL.

A. genuinum Hauck. - Cherbourg. HerB. J. B. de Bruxelles.

\section{G. latifolium Born.}

$=$ G. corneum pristoides J. Ag. - G. corneum variétés : capillaceum Grév.; Linnaei, hypnoides Ktz. ; pristoides J. Ag.; plumula Auct.

Petites-Dalles, Fécamp, Bénouville, Etretat, Antifer, Port-enBessin, Grandcamp. D. - Iles anglo-normandes. H. V. H. - Roscoff. Sir. - Z. I. anse du Minou, Corson. CRN. - Le Croisic. Fu. Biarritz, Guéthary, St. Jean de Luz. S. - San Vicente; Z. I. Gijon; Rivadeo. S.

\section{G. pectinatum Mont.}

Semble, au moins en partie, = G. latifolium Born, m'écrit De Toni.

Golfe de Gascogne. Born.

\section{G. pulchellum Ktz.}

$=G$. corneum pulchellum Grev. - Espèce incertaine, m'écrit De Toni. Semble, au moins en partie, = G. latifolium Born.

Cherbourg. Herb. J. B. de Bruxelles. - Iles anglo-normandes. H. V. H. - Morbihan. Herb. Zan. - Guéthary, San Vicente, Rivadeo, la Corogne. S.

A. setaceum Batt. - Iles anglo-normandes. H. V. H.

G. pusillum (Stackh.) Le Jol.

$=G$. clavatum et $G$. intricatum Lamour. - G. corneum clavatum Grev. - G. corneum caespitosum J. Ag. - G. pulvinatum Thur.

Gris-Nez; Wimereux, Pointe-aux-Oies et Croy ; Fécamp, Arromanches, Port-en-Bessin. D. - Quai Napoléon, jetées et fortifica- 
tions du port militaire, Z. M. LE JoL. - Iles anglo-normandes. H. V. H. - Roscoff. J. Сн. - Pointe N. E. de l'île de Bas. Melles V. et K. - Z. M. environs de Brest. CRN. - Pied de la falaise de Pen-Tir (Crozon). J. CH. - Z. S. sur rochers et Patelles, Biarritz. S. - San Vicente, Gijon, Rivadeo, la Corogne. S.

Forme très réduite, gazon sur roche de 2 à 3 millimètres de hauteur. Cap Figuier. Dét. Heydrich. J. Сн.

\section{Gelidium sesquipedale Thur.}

$=$ G. corneum sesquipedale Grev.

St. Malo, [station la plus sept ${ }^{\mathrm{te}}$ ]. Thur. - Laber-Ildut, Argenton, pointe de Corson, EP. Crn. - La Teste-de-Buch. Chantelat. Biarritz. Endress. - Guéthary, San Vicente, Gijon, Rivadeo, la Corogne. S.

Caulacanthus ustulatus (Mert.) Ktz.

= Gelidium J. Ag. - Gigartina Duby. - Hypnea Mont. Sphaerococcus Ag.

Z. I. Port-Vieux de Biarritz [station la plus septle]. Thur. - La Goureppe. J. Сн. - San Vicente, Rivadeo, la Corogne. S. - Le Ferrol. CoL.

\section{GIGARTINÉES}

Chondrus.

Gigartina.

Phyllophora.

Stenogramma.

Gymnogongrus.

Ahnfeltia.

Actinococeus.
Colacolepis. Sterrocolax. Callophyllis. Callocolax. Callymenia. Meredithia.

\section{Chondrus crispus (L.) Lyngb.}

EP. sur tout le littoral belge. Kx. - Tout le littoral de Dunkerque au Havre. D. - Z. M. et Z. I. Cherbourg. Le JoL. - Iles anglo-normandes. H. V. H. - Z M. et Z. I. Roscoff. Sir. - EP. abondante au Conquet. Langeron. - Z. M. environs de Brest. Crn. 
- Le Croisic. Fu. D. - Cap Ferret (Teste-de-Buch). Chantelat. Biarritz, Guéthary, St. Jean de Luz. S. - Castro (Asturies). Durieu. - San Vicente, Gijon, Rivadeo. S. - San Vicente, la Franca, Candas, la Corogne. Laz. - Santander, Concha de Artedo, le Ferrol. Cou. ENDRESS.

A. æqualis Lyngb. - EP. à Ostende. Donkelaer. - Biarritz.

B. angustifrons. - Z. I. Cherbourg. LE JoL.

C. cornens Ag. - Granville. Herb. J. B. de Bruxelles.

D. elongatus Duby. - Biarritz. Martens.

E. filiformis Duby. - Jersey 1902. H. V.H. - Biarritz. Endress.

F. incurvatus Lyngb. - EP. à Blankenberghe. Kx.

G. lacerus Lamour. - EP. à Nieuport. Kx. - Guernesey. Batt. - Banc de St. Mare. CRN.

H. latifrons. - Z. I. Cherbourg. LE JoL.

I. patens Turn. - Iles anglo-normandes. BАтт. - Belle-Isle. Herb. J. B. de Bruxelles.

J. planus Lamour. - EP. à Nieuport. Kx. - Banc de St. Marc. CRN.

K. sarniensis Turn. - Guernesey, Aurigny. Batr.

L. stellatus Turn. - Granville. HerB. J. B. de Bruxelles.

Gigartina acicularis (Wulf.) Lamour.

$=$ G. compressa Ktz. - Sphaerococcus Ag. - Sphaerococcus elongatus Delle Chiaje.

Bruneval, St. Jouin. Bernard. - Ste-Adresse. Dupray. - Luc, Port-en-Bessin. D. - Côtes du Calvados. Lew. - Z. M. et Z. I. Cherbourg. Chauv. - Iles anglo-normandes. H. V. H. - Granville. Chauv. - Roscoff. Sir. - EP. à Perkiridic. J. Ch. - Z. I. banc du Château, Ste-Anne. Crn. - Port et chenal du Croisic. FL. - La Govelle (Croisic). D. - La Teste-de-Buch. Chantelat. - Biarritz, Guéthary, St. Jean de Luz, San Vicente, Gijon, Rivadeo. S. La Corogne. Laz.

G. falcata (Hering.) J. Ag.

= Sphaerococcus Hering.

Bayonne. Darracq. - Biarritz. Postels, Martens. - Gijon. S. 
Gigartina mamillosa (Good. et Wood.) J. Ag.

= Mastocarpus Ktz. - Sphaerococcus Ag. - Chondrus Grev. Rhodymenia Aresch.

EP. à Blankenberghe. Kx. - EP. à Ostende. Landszweert. Gris-Nez, Audresselles. Giard. - Wimereux; toute la côte de Dieppe au Havre. D. - Cherbourg, Granville. Chauv. - Iles anglonormandes. H. V. H. - Roscoff. Sir. - Le Conquet. Langeron. Z. M. environs de Brest. CrN. - Le Croisic. FL. - Grande-Côte (Croisic). D. - Biarritz, Guéthary, St. Jean de Luz, San Vicente, Gijon. Rivadeo, la Corogne. S. - Candas. Laz. - Concha de Artedo (Asturies). Col.

Forme à lobes étroits (4-5 millimètres) laciniés. - Cherbourg. HeRB. J. B. de Bruxelles

Forme entièrement lisse. - Id. Id.

\section{G. pistillata (Gmel.) Stackh.}

= Sphaerococcus gigartinus Ag. - G. pectinata Bory.

Z. I. Equeurdreville, Nacqueville; Urville-Hague, sous les rochers de Landemer. Le JoL. - Iles anglo-normandes. H. V. H. Roscoff. SIr. - Perroch. Melles V. et K. - Z. I. anse du Minou, Bertheaume, Corson, Argenton. CRN. - Rochers de la Turballe, le Croisic. Fl. - EP. cap Ferret (Teste-de-Buch.) Chantelat. Biarritz, Guéthary, St. Jean de Luz, San Vicente, Gijon, Rivadeo, la Corogne. S. - La Franca, Candas. Laz. - Santander, Concha de Artedo (Asturies). Col.

A. pectinata J. Ag. - Gijon. Durieu. Cette forme tient le milieu entre G. Teedii et G. pistillata, d'après J. Ag.

Forme touffue et crépue, très dense. - Biarritz. J. Cн.

Forme non pistillée, ressemblant à une forme grêle du Chondrus crispus (état jeune?) - Morbihan, Bayonne. HerB. J. B. de Bruxelles. - Guéthary. J. Сн.

Sur la souche de ce Gigartina se trouvent parfois des rameaux stèriles, en forme de griffes ou pieds d'oiseau. Voir l'espèce suivante.

\section{G. Teedii (Roth.) Lamour.} thus Ktz.

= Sphaerococcus Ag. - Rhodomenia Grev. - Chondracan-

Granville, Cherbourg (rochers de Longlet). Pelvet in Herb. Chauv. - Iles anglo-normandes. H. V. H. - St. Malo. Herb. Len. - Roscoff. Sir. - Perroch. Melles V. et K. - Entrée du port de 
Brest, Camfrout. CrN. - Lorient. Herb. ZaN. - Morbihan. Leuièvre. - Port et chenal du Croisic. FL. D. - Biarritz, Guéthary, St. Jean de Luz. S. - Santander. CoL. - San Vicente, Gijon, Rivadeo, la Corogne. S.

A. angusta. - Brest. Herb. J. B. de Bruxelles.

Forme courte, robuste, stérile, très peu ramifiée. - Biarritz et Arotcha. Dét. Heydrich. J. CH.

Forme avec axe très élargi en lame membraneuse. - Biarritz. Lespinasse. - Disparait ou devient très rare en septembre. J. Сн.

Forme très réduite, crépue, de 2 à $3 \mathrm{ctm}$. - Biarritz. J. CH.

\section{Phyllophora Brodiæi (Turn.) J. Ag.}

EP. à Ostende. Kx. - Jersey, 1902. H. V. H. - Santander, San Vicente, Candas, la Corogne. Laz. - Côtes atlantiques de France. De Tons.

A. concatenata Rabh. - Pêché au large d'Ostende. (Fixé ou flottant ?) $\mathrm{Kx}$.

Ph. Heredia (Clem.) J. Ag.

Z. I. Laninon, St. Marc. CRN. - Fontarabie. CoL.

Ph. membranifolia (Good. et Wood.) J. Ag.

= Phyllotylus Ktz.

Gris-Nez. Giard. - Wimereux, Pointe-aux-Oies; Mesnilval. D. - Dieppe. Leturquier. - Petites-Dalles, Senneville, Fécamp, Yport. D. - Bénouville. Bernard. - Etretat. D. - Bruneval. Bernard. - Le Havre. Leturquier. - Port-en-Bessin, Grandcamp. D. - Côtes de la Manche. Desmaz. - Z. I. Hommet, Querqueville, Flamands. Le JoL. - Iles anglo-normandes. H. V. H. -- Aurigny.

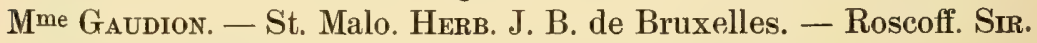
- Z. I. le Mingant, Bertheaume. CRs. - Le Croisic. FL. - San Vicente, la Corogne. LAz.

Ph. nervosa (D. C.) Grev.

= Halymenia Duby. - Delesseria Lamour. - Sphaerococcus Ag.

La Teste-de-Buch. Chantelat.

Espèce de la Méditerranée. 


\section{Phyllophora palmettoides J. Ag.}

\section{$=P h$. Brodiaei simplex Harv.}

Z. I. Cherbourg. LE JoL. - Iles anglo-normandes. H. V. H. Roscoff, EP. sur vieux stipes de Laminaria Cloustonii. J. CH. Z. I. anse du Minou, Bertheaume. CRN. - Z. I. Biarritz, Guéthary, St. Jean de Luz. S.

A. nicæensis J. Ag. - Biarritz. Born.

\section{Ph. rubens (L.) Grév.}

= Halymenia Duby. - Delesseria Lamour.

EP. à Ostende. W. Landszweert. - Gris-Nez. Giard. Wimereux, Pointe-aux-Oies, la Crèche. D. - Dieppe. Leturquier. - Fécamp, Yport. D. - Antifer, Bruneval. Bernard. - Le Havre. Dubec. - Langrune, Port-en-Bessin. Chauv. - Grandcamp. D. Z. I. Cherbourg. LE JoL. - Iles anglo-normandes. H. V. H. St. Malo. Herb. J. B. de Bruxelles. - Roscoff. Sir. J. CH. - Anse du Minou, Bertheaume. CRN. - Lorient. Herb. ZAN. - Morbihan. Herb. J. B. de Bruxelles. - Le Croisic. Fl. - La Govelle, plage Valentin (Croisic). D. - Cap Ferret (Teste-de-Buch). Chantelat. Biarritz, Guéthary, St. Jean de Luz, San Vicente, Gijon, Rivadeo. S. - Santander, Candas, Ares, le Ferrol. CoL.

\section{Stenogramma interruptum Ag.}

= Delesseria Ag. - St. europaeum, St. californicum Harv. Sphaerococcus bifidus ciliatus Ag. - Delesseria Ellisiae Lamour.

Iles anglo-normandes. H. V. H. - Roscoff. Melles V. et K. Dragué, Astan. Marty. - St. Pol de Léon. Dudresnay. - Dragué en rade de Brest; baie du Moulin-Blanc. CRN. - EP. Guéthary, un seul pied. S.

Forme stérile Crn. - Baie du Moulin-Blanc, EP. CRN.

De Toni p. 518: Stenogramma interrupta var. steritis Harv. = Rhodymenia linearis J. Ag. de la Nouvelle Zélande.

\section{Gymnogongrus Griffithsiæ (Turn.) Mart.}

= Chondrus J. Ag. - Gigartina Grév. - Sphaerococcus Ag. Audresselles; Wimereux, Pointe-aux-Oies, Croy. D. - Dieppe. Leturquier. - Fécamp, Yport. D. - Bruneval, St. Jouin. Bernard. - Luc. D. - Marigny-sur-mer. Bertot. - Port-en-Bessin, Grandcamp. D. - Z. M. Hommet, Ste-Anne, Querqueville. Le JoL. - Iles anglo-normandes. Jersey 1903. H. V. H. - Granville. HerB. J. B. de 
Bruxelles. - Z. M. Laninon, Kervallon, St. Marc. CrN. - Chenal du Croisic. FL. - Grande côte (Croisic). D. - San Vicente, Gijon, la Corogne. S.

A. minutus. - Z. I. Finistère. CRN.

Gymnogongrus norvegicus (Gunn.) J. Ag.

= Sphaerococcus Ag. - Chondrus Lamour. - Sphaerococcus crispus dubius Ag.

EP. à Ostende. Kx. - Gris.Nez, Audresselles, Wimereux. D. - Dieppe. Leturquier. - St. Valéry-en-Caux, Petites-Dalles, Senneville, Fécamp, Yport, Etretat. D. - Antifer, Bruneval. Bernard. - Quihot, Arromanches, Port-en-Bessin, Grandcamp. D. - Z. I. Cherbourg. Le Jol. - Iles anglo-normandes. H. V. H. - Carteret. Herb. Zan. - St. Malo. Delise. - Roscoff. Sir. - Perroch. Melles V. et K. - Z. I. Ste-Anne, le Minou. Crn. - Bretagne. Herb. Decaisne. - Le Croisic. Fl. - Grande côte, plage Valentin (Croisic). D. - Cap Ferret (Teste-de-Buch). Chantela T. - Biarritz, Guéthary, St. Jean de Luz. S. Lespinasse. - San Vicente, Gijon, Rivadeo, la Corogne. S.

G. patens (Grood. et Wood.) J. Ag.

= Chondrus crispus var. Ktz. - Sphaerococcus crispus patens Ag. - Chondrus celticus Ktz.

Roscoff. Sir. - Z. I. Corson, Laber-Ildut, Argenton. CRN. Bayonne. Blytt. - Biarritz. Endress. - Biarritz, Guéthary, St. Jean de Luz. S. Lespinasse.

\section{G. Torreyi (Ag.) J. Ag.}

= Sphaerococcus Ag. - Chondrus Grev.

Santander, St. Sébastien. Col.

Espèce de l'Amérique du N.

\section{Ahnfeltia plicata (Huds.) J. Ag.}

= Sphaerococcus Ag. - Gynnogongrus et Tylocarpus Ktz. - Gigartina Lamour. - Sphaerococcus aggregatus Ktz.

EP. sur tout le littoral belge. Kx. - Ostende. W. - Audresselles, Petites-Dalles, Fécamp, Yport, Etretat. D. - Bruneval, St. Jouin. Bernard. - Le Havre. Duboc. - Cherbourg, Granville. Chauv. Iles anglo-normandes. H. V. H. - St. Malo. Herв. J. B. de Bruxelles. 
- Roscoff, bord N. de l'île Verte, plage au S. de Ste-Barbe, etc. J. Сr. - Anse du Minou, Argenton. Crn. - Le Croisic. Fl. D. Biarritz, Guéthary, St. Jean de Luz, San Vicente, Gijon, Rivadeo, la Corogne. S. - St. Sébastien, Concha de Artedo (Asturies). Col.

\section{Actinococcus aggregatus Schm.}

Sur Gymnogongrus Griffithsiae. - Wimereux, Fécamp. D. Côtes de Calvados. Herb. Chauv. - Granville. Herb. J. B. de Bruxelles.

\section{A. peltiformis Schm.}

Sur Gymnogongrus norvegicus. - Fécamp, Yport, Port-enBessin. D. - Côtes du Calvados. Herb. Chauv. - Jersey 1903. H. V. H. - Roscoff, Perroch. Melles V. et. K. - Biarritz, Guéthary, St. Jean de Luz, San Vicente, Gijon, Rivadeo, la Corogne. S.

* A. simplicifilum J. Ag.

Espèce de la Baltique. A rechercher sur Chondrus crispus.

^ A. subcutaneus (Lyngb.) Rosenv.

= Chaetophora membranifolia Lyngb. - Rivularia rosea Suhr. - A. roseus Ktz. - Chaetophora Lyngb.

Sur Phyllophora Brodiaei. - Torquay. BAтт.

\section{* Colacolepis decipiens Schm.}

Sur Phyllophora Heredia. Probablement partout où l'on trouve la plante nourricière.

\section{G. incrustans Schm.}

Sur Phyllophora rubens. Probablement partout où l'on rencontre sa plante nourricière. Sur mes échantillons d'herbier : île Callot, Roscoff, Belle-Isle, Vannes. Voir à Phyllophora. - Cherbourg, Nacqueville. Hвrв. Thur. - Jersey 1903. H. V. H.

Savoir si les némathécies de Phyllophora membranifolia ne seraient pas un Colacolepis.

\section{Sterrocolax decipiens Schm.}

Sur Ahnfeltia plicata. - Cherbourg, Granville. Herb. LEN. Iles anglo-normandes. Jersey 1902. H. V. H. - Roscoff, chenal de l'île Verte. J. CH. - Dans l'exsiccata Crouan, nº 199, exemplaire 
du Dr Van Heurck : Laninon, le Minou, le Conquet etc... - La Goureppe, Guéthary. J. Сн.

\section{Callophyllis flabellata Crn.}

= Call. laciniata var : flabellata Hook.

Roscoff. Sir. - San Vicente de la Barquera. Laz. - Batterie du Fer-à-Cheval, Kervallon. CRN.

C. laciniata (Huds.) Ktz. Hook.

= Sphaerococcus Lyngb. - Rhodymenia Grev. - Delesseria

Dieppe. Desmaz. - Bruneval. Bernard, Dupray. - Z. I. Hommet, Querqueville, Digue; souvent EP. LE JoL. - Iles anglonormandes. H. V. H. - Roscoff. Sir. - EP. à Perkiridic. J. Cн. Plages d'Ouessant, le Conquet. Langeron. - Z. I. banc du Château, Laber-Ildut, Argenton. CrN. - Z. I. le Croisic. Fr. - Biarritz, Guéthary, St. Jean de Luz, San Vicente, Gijon, Rivadeo, la Corogne. S. - La Franca, Candas. Laz. -- St. Sébastien, Santander, Concha de Artedo, Cudillero. CoL.

A. immarginata Chauv. - Cherbourg. Chauv.

Forme avec bords plus ou moins tuberculeux et laciniés.

\section{Gallocolax neglectus Schmtz.}

Sur Callophyllis laciniata. - Barfleur. Herb. J. B. de Bruxelles. - Cherbourg. Herb. Len. - Jersey 1903. H. V. H. - Carantec, Roscoff, Guéthary. J. Сн.

\section{Gallymenia microphylla Zan.}

$=$ C. reniformis Ardiss.

Jersey 1903. H. V. H. - Pointe de l'île Callot, à très basse mer. J. CH.

Ardissone donne cette espèce croissant dans l'Atlantique, échappée de la Méditerranée.

\section{Polyides J. Ag.}

$=$ Rhodomenia J. Ag. - Sphaerococcus ? Ktz.

La Corogne. Solier. 
Callymenia reniformis (Turn.) J. Ag.

= Halymenia Ag. - Rhodomenia Hook. - Iridaea Bory. Sarcophyllis lobata Ktz.

Z. I. Hommet, Digue, île Pelée; EP. baie Ste-Anne. Le JoL. Iles anglo-normandes. H. V. H. - Roscoff. Sir. - Dragué à Astan. Melles V. et K. - EP. à Perkiridic. J. CH. - Le Croisic. FL. - EP. un seul exemplaire à Guéthary ; San Vicente, Rivadeo. S.

A. Ferrarii (Lamour.) J. Ag. (= Delesseria Lamour. - Iridaea Hilliana Grev.) - Cherbourg. Herв. J. B. de Bruxelles. - Iles anglo-normandes. H, V. H. - Porspoder. CRN.

\section{Meredithia microphylla J. Ag.}

\section{= Callymenia J. Ag. non Zan. - Halymenia Desmaz.}

Z. I. Cherbourg. Le JoL. - Jersey 1902. H. V. H. - Ile Callot, pointe N., tout à fait à la limite inférieure des plus basses mers. J. Сн. - Roscoff. Sir. - Z. I. Ste-Anne, Bertheaume, St. Mathieu. CRN. - San Vicente de la Barquera. Laz.

\section{RHODOPHYLLIDÉES}

Cystoclonium.

Catenella.

Euthora.
Rhodophyllis.

Solieria.

Gystoclonium purpurascens (Huds.) Ktz.

= Sphaerococcus Ag. - Gigartina Lamour. - Gracilaria Grev. - Hypnea Harv.

EP. à Ostende. W. - EP. à Nieuport et à la Panne. Kx. Blanc-Nez, Gris-Nez, Audresselles, Wimereux, Boulogne, le Portel et tout le littoral de la Seine inférieure et du Calvados. D. - Z. I. et souvent EP. à Cherbourg. Le Joc. - Iles anglo-normandes. H. V. H. - Roscoff. Sir. - Ile de Bas, chenal de l'Tle Verte. J. CH. - Astan. Melles V. et K. - Le Conquet. Langeron. - Banc du Moulin-Blanc, Ste-Anne, Bertheaume. CRN. - Le Croisic. FL. D. - La Corogne. Laz.

A. capillacenm Ag. - St. Malo. Herb. J. B. de Bruxelles.

B. cirrosum Harv. - Arromanches. Herb. J. B. de Bruxelles. 
- Roscoff, chenal de l'île Verte ; falaise de Pen-Tir (Crozon). J. Ch.

- Biarritz, St. Jean de Luz. Lespinasse.

C. scorpioideum Ag. - Luc. LEN.

Adulte, ne dépassant pas $10 \mathrm{ctm}$. - Arromanches. HerB. J. B. de Bruxelles.

Gatenella Opuntia (Good. et Wood.) Grev.

= Halymenia Ag. - Chondria Hook. - Gigartina Lamour. - Lomentaria Gaill.

Sur pilotis du port d'Ostende. Kx. - Calais. Desmaz. - GrisNez; Wimereux, la Rochette et Croy ; Fécamp, Yport, Etretat. D. Quais du Havre. Dupray. - Longues, Port-en-Bessin, Cherbourg. Chauv. - Z. S. quai Napoléon, Hommet. Le Jol. - Granville. BrÉB. - Iles anglo-normandes. H. V. H. - St. Malo. Herb. J. B. de Bruxelles. - Couvrant de grandes surfaces de roche, île Callot. J. CH. - Roscoff. Sir. Melles V. et K. - Z. S. environs de Brest.CRn. Belle-Isle. Herb. J. B. de Bruxelles. - Z. S. le Croisic. Fl. — Entrée des grottes de la grande côte (Croisic). D. - La Teste-de-Buch. Chantelat. - Guéthary, San Vicente, Gijon, Rivadeo. S. Portugalete, le Ferrol. CoL.

\section{Euthora cristata (L.) J. Ag.}

= Rhodymenia Grev. - Callophyllis Ktz.

San Vicente de la Barquera. LAz.

Atlantique du Nord.

\section{Rhodophyllis appendiculata J. Ag.}

= Rhodymenia bifida ciliata Harv.

Côtes du Calvados. Len. - Iles anglo-normandes. H. V. H. St. Malo. Herb. J. B. de Bruxelles. - Bane de St. Marc, EP. Crn. - Morbihan. Herв. J. B. de Bruxelles.

A. circinans Crn. - Z. I. Fort Mingant. CRN.

Forme réduite à 2-3 ctm. - Barfleur. Herb. J. B. de Bruxelles.

Rh. bifida (Good. et Wood.) Ktz.

= Sphaerococcus Ag. - Rhodomenia Grev.

Wimereux, Croy. D. - Boulogne, à la Roche-Bernard. Giard. - Petites-Dalles, Senneville, Fécamp, Yport, Etretat. D. - Luc. Bernard. - Langrune. Chauv. - Arromanches. Bertot. - Porten-Bessin. D. - Cherbourg. Le Jol. - Iles anglo-normandes. H. V. H. 
- Roscoff. Sir. - Banc du Château, Laninon. Crv. - Z. I. le Croisic. FL. D. - Biarritz. J. CH. - Guéthary. S. - San Vicente de la Barquera. Laz.

Frondes larges. - Luc, Arromanches. Chauv.

Frondes filiformes.

Solieria chordalis (Ag.) J. Ag.

= Sphaerococcus Ag. - Delesseria Ag. - Gracilaria Grev. Plocaria Endl.

Pointe du Bendic. Crs. - Morbihan. Thur. - Vannes. Herb. J. Сн. - Le Croisic. Fu. D. - Ile de Noirmoutier. Bons. - Gijon. S.

Forme très feuillée et forme à rameaux nus, selon l'hiver et l'été.

\section{SPHÉROGOGGACÉES}

Sphærococcus.

Gracilaria.

Calliblepharis.
Hypnea.

Gelidiopsis.

Sphærococcus coronopifolius (Wood. et Good.) Ag.

= Gelidium Lamour. - Rhynchococcus Ktz. - Plocamium vulgare Delle Chiaje.

EP. à Bruneval. Dupray. - Cricqueville. Bertot. - Portbail. Herb. J. B. de Bruxelles. - Z. I. Hommet, Querqueville, Digue; souvent EP. LE Jou. - Iles anglo-normandes. H. V. H. - St. Malo. Herb. J. B. de Bruxelles. - Roscoff. Sir. - EP. à Perkiridic. J. Ch. -- Le Conquet. Langeron. - Z. I. Porspoder, Laber-Ildut, Argenton. CRN. - Le Croisic. FL. - Z. I. Biarritz, Guéthary, St. Jean de Luz. S. - St. Sébastien. Col. - San Vicente, Gijon, Rivadeo, la Corogne. S. - Concha de Artedo (Asturies), Candas. CoL.

Forme capillaire. - Gijon. Durieu.

Gracilaria armata (Ag.) J. Ag.

Biarritz. BoRN. 


\section{Gracilaria compressa (Ag.) Grev.}

= Sphaerococcus Ag. - Plocaria Endl. - Gigartina Hook.

Grandcamp. D. — St. Vaast. LE JoL. - Iles anglo-normandes. H. V. H. - Granville. Herb. J. B. de Bruxelles. - Roscoff. Melles V. et K. - Banc de St. Marc. CRN. - Toulinguet (Camaret). Dét. Reinbold. J. Ch. - Morbihan. HerB. J. B. de Bruxelles. - Le Croisic. - Fu. D. - San Vicente, Santander, Candas. Laz.

\section{Gr. confervoides (L.) Grev.}

= Plocaria Mont. - Sphaerococcus Ag. - Gigartina Lamour. - Hypnaea J. Ag.

Sur les pierres et les coquilles à Nieuport (Fixé ?). Kx. - BlancNez, Gris-Nez, Audresselles, Wimereux, Boulogne, le Portel, Ault et tout le littoral de la Seine inférieure et du Calvados. D. Cherbourg. Le JoL. - Iles anglo-normandes. H. V. H. - Roscoff. Sir. J. CH. - Laninon, le Minou. CrN. - Le Croisic. Fl. D. Bassin d'Arcachon, pointe d'Aiguillon. Chantelat. - Biarritz, San Vicente, Gijon, Rivadeo, la Corogne. S. - Santander, Candas. LAz. - Concha de Artedo (Asturies). Col.

A. divaricata (5 ctm.) - Granville. Len.

B. geniculata Hook. (avec vrilles). - Granville, Luc. CHauv.

C. procerrima Hook, Atteignant $50 \mathrm{ctm}$. - Côtes du Calvados. Chauv. - Cherbourg. Herb. J. B. de Bruxelles. - Ile de Bas. Melles V. et K. - Morbihan. Herв. J. B. de Bruxelles. - Bassin d'Arcachon, pointe d'Aiguillon. Chantelat.

Filiforme. - Le Havre. Martens.

Forme courte et circinée (5 ctm.) - Cherbourg. Herb. J. B. de Bruxelles.

Varie en un même lieu de la forme très ramifiée, longue de $25 \mathrm{ctm}$. et davantage, à la forme capillaire réduite jusque $5 \mathrm{ctm}$. - Arromanches.

\section{Gr. dura (Ag.) J. Ag.}

= Sphaerococcus Ag. - Plocaria Endl. - Gigartina Durvillaei Lamour.

Boulogne. Desmaz. - Anse du Minou, côte W., Porspoder. Crn. - Golfe de Gascogne. Born. 


\section{Gracilaria multipartita (Clem.) Harv.}

= Sphaerococcus Ag. - Sphaerococcus polycarpus Grev.

Roscoff, Perroch. Melles V. et K. - Z. I. entrée du port de Brest, Laninon. CRN, - Morbihan. LeN. - Le Croisic. FL. D. - Bayonne (de 5 ctm.) Darracq. - Biarritz, Guéthary, St. Jean de Luz, San Vicente, Gijon. S.

A. angustissima. - Roscoff, Perkiridic, courants de basse mer sur le sable. Ј. Сн.

\section{Galliblepharis ciliata (Huds.) Ktz.}

= Delesseria Lamour. - Halymenia Gaill. - Fucus Huds. - Sphaerococcus Ag. - Rhodomenia Grev.

EP. à Nieuport. Kx. - Roche-Bernard (Boulogne). Giard. EP. à Ault et à Cayeux; tout le littoral du Tréport au Havre; Langrune, Arromanches, Port-en-Bessin et Grandcamp. D. - Z. I. Hommet, Querqueville; souvent EP. LE JoL. - Iles anglo-normandes. H. V. H. - Roscoff. Sir. - Entrée du port de Brest, Laninon. CRN. - Morbihan (jusque $45 \mathrm{ctm}$. de longueur). Lelièvre. - Le Croisic. Fu. D. - La Teste-de-Buch. Chantelat. - Z. I. Biarritz, Guéthary, St. Jean de Luz, San Vicente, Gijon, Rivadeo, la Corogne. S. Santander, Comillas, la Franca, Candas. Laz. - Bilbao, Portugalete, St. Sébastien, Cudillero. CoL.

Varie depuis la forme large à bords presque entiers jusqu'à la ramification échevelée.

A. linearis. - Granville. Len. - Biarritz. Endress. - Segments larges de 5 à 6 mill. pieds nombreux sous la villa Belza à Biarritz, en mai; disparus en septembre. J. Сн.

B. palmata Grev. - Bayonne. RoMPeL.

C. spinosa. - Banc de St. Marc. CrN. - Biarritz. Endress.

G. jubata (Good. et Wood.) Ktz.

= Sphaerococcus Grev. - Rhodomenia Grev.

EP. à Ostende. W. - Barfleur. LEN. - Z. M. Cherbourg. Le JoL. - Iles anglo-normandes. H. V. H. - St. Malo. Melle LeBrun. - Roscoff. Sir. - Le Conquet. Langeron. - Z. I. Ste-Anne. Crn. - Le Croisic. Fu. - Biarritz, Guéthary, St. Jean de Luz, San Vicente, Rivadeo, la Corogne. S. - Gijon. Durieu. - La Franca, Candas. Laz.

Cette espèce et la précédente offrent des formes si variables et 
si rapprochées qu'on ne peut les distinguer sinon par les fructifications. (Lespinasse).

Capillaire, longueur $8 \mathrm{ctm}$. - Cherbourg. HerB. J. B. de Bruxelles.

Sublinéaire et scorpiö̈de. - Anse du Minou. CRN.

Plus petite que $5 \mathrm{ctm}$. - Iles anglo-normandes. BRÉB.

Larges frondes, à lobes fins et nombreux.

Frondes cylindriques, sans lobes.

Frondes cylindriques à petits lobes spiniformes.

Lobes ramifiés et plus ou moins chevelus.

Hypnea musciformis (Wulf.) Lamour.

Biarritz. Lespinasse. - Biarritz, Guéthary, St. Jean de Luz, San Vicente, Gijon. S. - Candas, la Corogne. Laz.

Gelidiopsis pannosa (Grun.) Schmitz.

$=$ Gelidium Grun.

Voûtes des grottes marines de Biarritz. BorN. - San Vicente. S.

\section{RHODYMÉNIÉES}

Rhodymenia.

Cordylecladia.

Lomentaria.

Gastroclonium.

Champia.
Chylocladia.

Plocamium.

Chrysymenia.

Fauchea.

Rhodymenia ligulata Zan.

$=$ Sphaerococcus Ktz. - Sph. Meneghinii Ktz.

San Vicente de la Barquera, Llanes, Candas, la Corogne. Laz. Espèce de l'Adriatique.

Rh. palmata (L.) J. Ag.

= Halymenia Ag. - Sphaerococcus Ktz.

EP. à Ostende et sur tout le littoral. Kx. - Blanc-Nez, Gris-Nez, Audresselles, Wimereux, le Portel et tout le littoral de la Seine inf. et du Calvados. D. - Z. M. et Z. I. Cherbourg. Le Joz. - Iles 
anglo-normandes. H. V. H. - Z. M. Roscoff, très polymorphe. J. CH. - Le Conquet. Langeron. -- Z. I. environs de Brest. CRn. - Z. I. le Croisic. Fu. D. - San Vicente, Gijon; EP. à Rivadeo, de petite taille; la Corogne, EP. S. - La Franca. Laz. - Cudillero (Asturies), Candas, le Ferrol. Cou.

A. laciniata. - Iles anglo-normandes. H. V. H.

B. marginifera Harv. - Cherbourg. Chauv. - Jersey 1903. H. V. H.

C. sarniensis (Mert.) J. Ag. (= Rhod. sarniensis Grev. - Haly. menia sarniensis Duby). - EP. entre Nieuport et la Panne. Kx. - La Corogne. Laz. - Barfleur. Desmaz. - Z. M. Cherbourg. Chauv. - Iles anglo-normandes. H. V. H. - Granville. Len. Roscoff. Sir. - Z. M. environs de Brest. CRN.

Varie entre 5 et $20 \mathrm{ctm}$. de longueur.

D. sobolifera J. Ag. (= Rhodomenia sobotifera Grev. - Halymenia sobolifera Ag. - Callophyllis et Sphaerococcus soboliferus Ktz.) - Iles anglo-normandes. H. V. H. - Iles Chausey. HerB. J. B. de Bruxelles. - Environs de Brest. CrN. - San Vicente de la Barquera. Laz.

Forme triangulaire, attachée par un sommet, richement frangée sur la base opposée.

Forme presque entièrement capillaire et très ramifiée.

\section{Rhodymenia palmetta (Esp.) Grev.}

= Halymenia Lamour. - Sphaerococcus Ag. - Halymenia nicueensis Duby.

EP. sur le littoral belge. Kx. - Dieppe. Desmaz. - Côtes du Calvados, Cherbourg. Chauv. - Arromanches. Herb. Zan. - Z. I. Hommet, Querqueville, Digue. Le Jot. - Iles anglo-normandes. H. V. H. - Z. I. le Mingant, Bertheaume. CrN. - Chenal du Croisic. Fu. - Grande plage (Croisic). D. - - Cap Ferret (Teste-de-Buch). Chantelat. - Z. I. Biarritz, Guéthary, St. Jean de Luz, San Vicente, Gijon, Rivadeo, la Corogne. S.

A. Elisiæ Chauv. (= Rhod. nicaeensis Holm. - Rhod. palmetta nicaeensis J. Ag.) Voir Ardissone Rhod. palmetta et Rhod. nicueensis. De Toni p. 515. Agardh, Epicrisis, p. 330 et 218. Ressemble beaucoup à Phyllophora palmettoides nicaeensis (Born). - EP. à Ostende. Kx. - Gris-Nez; Wimereux, Pointe-aux-Oies, Croy ; Mesnilval. D. - Dieppe. Desmaz. — St. Valéry-en-Caux, Senneville, 
Fécamp. D. - Langrune, Port-en-Bessin. Chauv. - Dragué à Bernières. D. - Arromanches. Len. - Batterie du Diable, Crozon, Bertheaume. Crn. - La Goureppe. J. Ch.

B. latifolia Crn. (= Rhod. palmetta crassiuscula Turn.) Fécamp. D. - Yport. Bernard. - Etretat, Bénouville. D. - Pointe de Corson, Laber-Ildut, Argenton. CRN. - Biarritz. J. СH.

Cordylecladia erecta (Grev.) J. Ag. Grev.

= Sphaerococcus Grev. - Gigartina Hook. -- Gracilaria

Wimereux, Pointe-aux-Oies. Cornu. - Bernières. Chauv. Z. I. Cherbourg. Le JoL. - Iles anglo-normandes. H. V. H. - Anse du Minou, Bertheaume, Laber-Ildut. CRN.

Lomentaria articulata (Huds.) Lyngb.

= Chondria Ag. - Chylocladia Grev.

EP. et sur les pierres, Ostende, Nieuport, la Panne. Kx. - Z. S. Gris-Nez, Audresselles, Wimereux, et toutes les côtes de la Seine inférieure. D. - Cherbourg, Granville. Chauv. - Iles anglo-normandes. H. V. H. - Roscoff. Sir. - Le Conquet. Langeron. - Z. I. environs de Brest. CrN. - Belle-Isle. Lloyd. - Morbihan. Herb. J. B. de Bruxelles. - Le Croisic. FL. D. - La Rochelle (dimension très réduite). D'Orbigny. - Z. I. Biarritz, Guéthary, St. Jean de Luz. S. - Comillas, Candas. Laz. - Le Ferrol. Col. - San Vicente, Gijon, Rivadeo ; EP. sur Laminaria Cloustonii, la Corogne. S.

Gastroclonium kaliforme (Good. et Wood.) Ardiss.

= Chondria Ag. - Chylocladia Hook. - Gigartina Lamour.

- Lomentaria patens Ktz.

EP. à Ostende et à Nieuport. W. - Dieppe. Leturquier. Yport, Bénouville. Bernard. - Quihot. D. - Cricqueville. Bertot. - Grandcamp. D. - Calvados (jusque $30 \mathrm{ctm}$. de longueur). Len. Luc, Langrune, Arromanches, Port-en-Bessin, Cherbourg. Chauv. Iles anglo-normandes. H. V. H. - Roscoff. Sir. - Santec. Miciol. - Z. I. banc de St. Marc, Laninon. CrN. - Le Croisic. FL. D. La Teste-de-Buch. Chantelat. - Biarritz, Guéthary, St. Jean de Luz. S. - San Vicente de la Barquera. Laz.
A. æstivale Le Jol. - Z. M. Cherbourg. Le JoL.
B. helminthoides Le Jol. - Z. I. Cherbourg. LE JoL. 
C. squarrosum (Ktz.) J. Ag. ( $=$ Chondria polyclados Ktz. Lomentaria squarrosa, L. torulosa, L. phalligera Ktz. - Chylocladia squarrosa Le Jol. - Lomentaria kaliformis De Not). - Sur Polyides et Fastigiaria, Cherbourg. LE Jox. - Iles anglo-normandes. H. V. H. - Environs de Brest. Crn. - Le Croisic. Fr. - Biarritz, Guéthary, St. Jean de Luz, la Corogne. S.

D. torulosum J. Ag. (=Lomentaria torulosa Bonnem. - De Toni donne cette forme comme syn. de la var. squarrosum. Crouan distingue les deux variétés. Sauvageau les cite comme deux espèces). - Banc de St. Marc. CRn. - Biarritz. Thur. - Z. I. Guéthary. S.

E. vernale Le Jol. - Z. M. et Z. I. entrée du port militaire, plages Napoléon et des Bains. LE JoL.

F. verticillatum J. Ag. - Banc de St. Marc, Ste-Anne. CrN.

Gastroclonium ovale (Huds.) Ktz.

= Chondria Ag. - Lomentaria Endl. - Chylocladia Hook.

EP. à Ostende. Herb. J. B. de Bruxelles. - Pointe-aux-Oies, Yport. D. - Bénouville. Bernard. - Quihot. D. - Langrune. Chauv. - Port-en-Bessin, Grandcamp. D. - Côtes du Calvados. Len. - Cherbourg. Chauv. - Iles anglo-normandes. H. V. H. Roscoff. Sir. - St. Pol de Léon (Pempoul). Miciol. - Z. I. banc du Château, Ste-Anne. Crn. - Le Croisic. Fl. - Grande côte (Croisic). D. - Biarritz, Guéthary, St. Jean de Luz, Rivadeo, la Corogne. S. - Le Ferrol. Col.

A. microphyllum Crn. - Arromanches, Belle-Isle. Lloyd.

B. subarticulatum Crn. - Environs de Brest. CrN.

Très feuillé au printemps, réduit aux rameaux vers l'automne. Forme à folioles larges, orbiculaires.

\section{G. reflexum (Chauv.) Ktz.}

= Lomentaria Chauv. - Chylocladia Zan. Len. - Lomentaria pusilla Ktz.

A été trouvé une fois en EP. à la Panne. Kx. - Fécamp, Yport, Etretat, St. Jouin, Courseulles. D. - Luc, Arromanches, Port-enBessin. Chauv. - Iles anglo-normandes. H. V. H. - Z. I. anse du Minou. Crn. - La Goureppe (Guéthary). S. 


\section{Champia parvula (Ag.) J. Ag.}

= Lomentaria Gaill. - Chondria Ag. - Chylocladia Hook. - Chondria implexa et Lomentaria intertexta Chauv.

EP. à Ostende. W. - Wimereux, Croy; St. Aubin-sur-Mer. D. - Langrune. Chauv. - EP. à Arromanches. Bertot. - Grandcamp. D. - Z. I. Cherbourg. LE JoL. - Iles anglo-normandes. H. V. H. Roscoff. Srr. - Z. I. environs de Brest. CrN. - Le Croisic. FL. D. - Biarritz. Endress. - De petite taille à Guéthary, San Vicente, Gijon, la Corogne. S.

Ghylocladia clavellosa (Turn.) Grév.

= Chrysymenia J. Ag. - Lomentaria Le Jol. -- Chondria Ag. De 3 à 4 cent. seulement, attaché aux pierres à Ostende. Landszweert. - Wimereux, Fécamp, Yport. D. - Etretat, St. Jouin. Bernard. - Luc, Langrune. Chauv. - Maisy. Bertot. - Côtes du Calvados. Len. - Z. I. Cherbourg. Le Jol. - Iles anglo-normandes. H. V. H. - Roscoff. Sir. - Dragué à Astan. Melles V. et K. - EP. à Perkiridic. J. Ch. - Environs de Brest. CrN. - Le Croisic. Fl. D. - La Teste-de-Buch, pointe d'Aiguillon. Chantelat. — Gijon, Rivadeo; de petite taille. S.

A. corymbifera Crn.

B. patens Crn.

C. pyramidalis Thur. - Hommet, Querqueville. LE JoL.

Ch. rosea Harv.

= Lomentaria Thur. - Chrysymenia Harv.

Rade de Brest, dragué. CRN.

\section{Plocamium Binderianum Ktz.}

$=P l$. coccineum Binderianum. Se relie au type par des intermédiaires selon Van Heurck.

Jersey 1902. H. V. H.

\section{Pl. coccineum (Huds.) Lyngb.}

=Delesseria Plocamium Ag. - Delesseria Ag. - Pl. vulgare Lamour.

EP. sur tout le littoral belge; Ostende. Mac-Leod. - Dunkerque. Desuaz. - Blane-Nez, Gris-Nez, Audresselles, Wimereux, Boulogne, Ault et toute la côte de la Seine inf. et du Calvados. D. - Granville, 
Arromanches, Cherbourg. HerB. ZAN. - Iles anglo-normandes. H. V. H. - St. Malo. Delise. - Roscoff. Sir. - EP. à Perkiridic. J. CH. - Environs de Brest. CRN. - Le Croisic. Fl. D. - La Teste-de-Buch. Chantelat. - Biarritz, Guéthary, St. Jean de Luz, San Vicente, Gijon, Rivadeo, la Corogne. S. - Candas. Laz.

A. angustifrons Le Jol. - Z. I. Cherbourg. Le JoL. - Iles anglo-normandes. BatT. - Finistère. CRN.

B. latifrons Le Jol. - Z. I. et souvent EP. à Cherbourg. LE JoL. - Iles anglo-normandes. BatT.

Le Jolis et Batters ne donnent que les deux variétés angustifrons et latifrons, et ne supposent pas un Plocamium coccineum typique.

C. uncinatum J. Ag. (=Pl. subtile $\mathrm{Ktz},-P l$. uncinatum Ktz. - Pl. coccineum subtile Lyngb.) - EP. à Nieuport. Kx. - Wimereux, Mesnilval. D. - Bruneval. Bernard. - Portbail. Herb. J. B. de Bruxelles. - Plages Napoléon et des Bains, souvent EP. LE JoL. - Iles anglo-normandes. H V. H. - Mer basse au Cerf. J. Сн. Laninon, Ste-Anne, EP. CRN. - Biarritz, Guéthary. J. Сн.

\section{Ghrysymenia Chiajeana Menegh.}

Biarritz, limite nord. Bonv. - Sur Araignée de mer à Guéthary. S.

Gh. Uvaria (L.) J. Ag.

= Gastroclonium Ktz. - Chondria Ag.

Le Ferrol. Col.

Gh. ventricosa J. Ag.

$=$ Dumontia Lamour.

EP. à Guéthary. S.

\section{Fauchea microspora Born.}

Sur Araignée de mer à Guéthary. Limite sud de l'espèce. S.

$$
\text { F. repens (Ag.) Mont. }
$$

= Sphaerococcus Ag. - Chondrus Grev. - Plocaria Endl.

San Vicente de la Barquera. Laz. - La Corogne. Bohn. et Col. ce dernier d'après F AUchÉ. 


\section{DELESSÉRIÉES}

Nitophyllum.

Gonimophyllum.

Delesseria.
Hypoglossum.

Apoglossum.

Pteridium.

\section{Nitophyllum alliaceum Crn.}

$=N$. punctatum alliaceum J. Ag. - N. Pollexfenii Harv. Rade de Brest, dragué et EP. CRN.

\section{N. Bonnemaisonii Grev.}

\section{= Delesseria Grev. - Aglaophyllum Endl.}

Toute la côte de Normandie. Bonnemarson. - Rochers, pointe de Querqueville. Thur. - Iles anglo-normandes. H. V. H. - EP. à Barfleur, Cherbourg, Granville. CHauv. - Roscoff. Melles V. et K. Z. I. rade de Brest, Melon, fort Mingant, Porspoder. Crs. - Grande côte (Croisic). D.

\section{N. Gmelini Grev.}

$=$ Delesseria Grev.

EP. près de Nieuport. Kx. - Gris-Nez. GiaRD. - Mesnilval, St. Valéry-en-Caux, Petites-Dalles, Senneville, Fécamp, Yport, Etretat, St. Jouin, Langrune; EP. à Arromanches. D. - Luc, Porten-Bessin. Chauv. - Réville. Le Jol. - Iles anglo-normandes. H. V.H. - Rivadeo. S. - Z. I. banc du Moulin-Blanc, Kervallon. Crn. - Belle-Isle. Herb. J. B. de Bruxelles. - Chenal du Croisic. FL. - Grande côte (Croisic). D.

\section{N. Hilliæ Grev.}

= Delesseria Grev. - Delesseria Bonnemaisonii Ag. - Nit. ulvoideum Hook.

Z. I. Cherbourg. LE JoL. - Iles anglo-normandes. H. V. H. Roscoff. SIr. - Ile Callot. Melles V. et K. - Le Cerf ; EP. à Perkiridic. J. Сн. - Z. I. entrée du port de Brest, Ste-Anne, Laber-lldut. Crs. - Pen-Tir (Crozon). J. Ch. - Rivadeo, la Corogne. S. 
Nitophyllum laceratum (Gmel.) Grev.

= Halymenia Duby. - Delesseria Ag.

Roche-Bernard (Boulogne); EP. à Cayeux; toute la côte de la Seine inf., Quihot, Langrune, Port-en-Bessin, Grandcamp. D. - Côtes du Calvados. Len. - Z. I. Cherbourg. Le JoL. - Iles anglo-normandes. H. V. H. - Carteret. Herb. Zan. - St. Malo. Herb. J. B. de Bruxelles. - Roscoff. Sir. - Le Conquet. Langeron. - Laninon, le Mingant. Crn. - Pen.Tir (Crozon). J. Ch. - Le Croisic. FL. D. La Teste-de-Buch. Chantelat. - Biarritz, Guéthary, St. Jean de Luz, San Vicente, Gijon, Rivadeo, la Corogne. S. - Santander, la Franca. Laz. - St. Sébastien, Candas. CoL.

A. carnosum Le Jol. - Sur Ahnfeltia, au-dessous du fort du Hommet. Le Jol.

B. dichotomum Ktz. - Iles anglo-normandes. H. V. H.

C. Iatifolium (=N. Smithii Crn. non Hook et Harv.) - LaberIldut, Porspoder. CRN.

D. lobatum Ktz. - Iles anglo-normandes. H. V. H.

N. punctatum (Stackh.) Grev.

= Aglaophyllum Aresch. - Delesseria Ag.

Iles anglo-normandes. H. V.H. - Roscoff. SiR. - EP. grande côte (Croisic). D. - Biarritz, Guéthary, St. Jean de Luz, San Vicente, Gijon, Rivadeo, la Corogne. S.

A. ocellatum (Lamour.) J. Ag. (= Delesseria ocellata Lamour. - Nit. ocellatum Grev.) - Fécamp. Leturquier. - Bruneval. Bernard. - Z. I. Port-en-Bessin, Cherbourg. Chauv. - Cricqueville (Grandcamp). Bertot. - Querqueville. Herb. ZaN. - Côtes de la Manche. BRÉB. - Iles anglo-normandes. H. V. H. - St. Malo. Melle Lebrun. - Roscoff. Sir. - Banc du Moulin-Blanc. Crn. Chenal du Croisic Fr. - Biarritz. Postels. - La Corogne. Col.

B. ulvoides J. Ag. - Barfleur. Herb. J. B. de Bruxelles. - Z. I. Laninon, Porspoder. CRN.

\section{N. reptans Crn.}

Cherbourg. LE JoL. - Iles anglo-normandes, sur Lithothamnion polymorphum. H. V. H. - Rade de Brest, dragué. CRN. 
Nitophyllum Sandrianum (Zan.) Crn.

= Delesseria Zan. - Aglaophyllum Ktz.

Rade de Brest, dragué. CRN.

\section{N. uncinatum (Turn.) J. Ag.}

= Delesseria lacerata uncinata Ag. - D'après Batters différent du $N$. laceratum uncinatum Grev.

Rochers de la pointe de Querqueville. Le JoL. - Iles anglonormandes. H. V. H. - Roscoff. Sir. - Dragué à Astan. Melles V. et K. - Rade de Brest, baie de Bertheaume, Porspoder, EP. CRN. - Le Croisic. Fl. - Biarritz, Guéthary, St. Jean de Luz, San Vicente, Gijon, Rivadeo, la Corogne. S. - St. Sébastien. CoL.

\section{N. venulosum Zan.}

Jersey 1904. Dét. Reinbold. H. V. H.

Signalé dans l'Adriatique, sur les côtes de Sardaigne, à Tanger, à Plymouth.

\section{N. versicolor Harv.}

= Aglaophyllum Ktz. - Cryptopleura heterocarpa Ktz. Halymenia heterocarpa Duby.

EP. Luc. Chauv. - Courseulles, Asnelles, Arromanches. Bertot. - Iles anglo-normandes. H. V. H. - Anse Tréménarch en Plouguerneau, Melon, EP. Crn. - San Vicente de la Barquera. Laz. Le Ferrol. CoL.

\section{Gonimophyllum Buffhami Batt.}

Parasite du Nitophyllum laceratum. - Jersey 1902. H. V. H.

Delesseria sanguinea (L.) Lamour.

= Hydrolapathum Stackh. - Wormskjoldia Spr.

EP. entre Nieuport et la Panne. Kx. - Dieppe, Fécamp. Leturquier. - Fécamp, Yport, Bénouville, Etretat. D. - Antifer, Bruneval, St. Jouin. Bernard. - Bernières. Morière. - Luc. Len. - Z. I. Hommet, Digue, Querqueville. LE JoL. - Iles anglo-normandes. H. V. H. - Roscoff. Sir. - Le Cerf, aux plus basses mers, ne découvrant jamais; EP. à Perkiridic. J. CH. - Z. I. batterie du Diable, le Mingant, Bertheaume, Argenton. CrN. - Belle-Isle. Herb. J. B. de Bruxelles. - Le Croisic. Ft. - Z. I. grande côte 
(Croisic). D. - EP. à San Vicente et Gijon; Z. I. à Rivadeo, la Corogne. S. - Llanes. Laz. - Candas, le Ferrol. Col.

Delesseria sinuosa (Good. et Wood.) Lamour.

$=D$. crenata $\mathrm{J}$. Ag.

EP. à Blankenberghe et à Ostende. Kx. - Dieppe, St. Valéryen-Caux, Fécamp. Leturquier. - Cherbourg. Le Jol. - Iles anglonormandes. H. V. H. - St. Malo. Herb. J. B. de Bruxelles. -Roscoff. Srr. - Plages d'Ouessant. Langeron. - Laber-Ildut, Melon, Argenton. Crn. - Le Croisic. Fl. - San Vicente, Candas, la Corogne. Laz. - Côtes de Galicie. Col.

\section{Hypoglossum Woodwardii Ktz.}

= Delesseria Hypoglossum Lamour. - D. lingulata Duby. $H$. lingulatum, $H$. concatenatum Ktz.

Du Gris-Nez à Boulogne, Wimereux, tout le littoral de la Seine inf. D. - Luc, Port-en-Bessin, Cherbourg. Chauv. - Z. I. Cherbourg. LE JoL. - Iles anglo-normandes. H. V. H. - Roscoff. Sir. Perroch. Melles V. et K. - Pen-Tir (Crozon). J. CH. - Le Croisic. FL. D. - Cap Ferret (Teste-de-Buch). Chantelat. - Biarritz, Guéthary, St. Jean de Luz, San Vicente, Gijon, Rivadeo, la Corogne. S. - Le Ferrol. CoL.

A. angustifolium Ktz. (= Hyp. minutum Ktz.) - Iles anglonormandes. H. V. H. - Roscoff, île Callot. Melles V. et K.

B. arborescens Crn. - Sous le Polygone et entrée du port militaire de Brest. CrN.

C. crispum Crn. (= Delesseria crispa Zan. - Hypogl.crispum Ktz.) - Jersey 1903. H. V. H. - Laninon, entrée du port de Brest, le Minou, Z. I. CRN.

D. filiforme Len. - Cherbourg. Hers. J. B. de Bruxelles.

E. glomeratum Chauv. - Côtes du Calvados. Lev. - Luc. Chauv. - Z. I. Cherbourg. Le Jol. - Laninon. CRN.

F. ovale J. Ag. - Iles anglo-normandes. H. V. H.

G. recurvatum Heydr. - Iles anglo-normandes. H. V. H.

Apoglossum ruscifolium (Turn.) J. Ag.

= Delesseria Ag. - Hypoglossum Ktz.

Dieppe, Fécamp. Leturquier. - Le Havre. Duboc. - Luc, Langrune. Chauv. - Arromanches, Port-en-Bessin. D. - Côtes du 
Calvados. Len. - Z. I. Hommet, Querqueville, Nacqueville. Le JoL. - Iles anglo-normandes. H. V. H. - St. Malo. Herb. J. B. de Bruxelles. - Roscoff. Sir. - Z. I. le Conquet. Langeron. - Z. I. Laninon, banc du Château. CRN. - Pen-Tir (Crozon). J. CH. - BelleIsle. Herb. J. B. de Bruxelles. - Le Croisic. FL. - Biarritz, Guéthary, St. Jean de Luz, San Vicente, Gijon, Rivadeo. S.

\section{Pteridium alatum (Huds.) J. Ag.}

= Delesseria Lamour. - Hypoglossum Ktz. - Hypoglossum carpophyllum Ktz.

EP. à Ostende, sur Laminaria. Kx. - Nieuport. W. - Gris-Nez, Audresselles. Giard. - Dieppe, St. Valéry-en-Caux, des PetitesDalles au Havre. D. - Côtes du Calvados. BRÉB. - Arromanches, Gatteville, Hermanville. Herb. Zan. - Port-en-Bessin. Chauv. Z. M. Cherbourg. Le JoL. - Iles anglo-normandes. H. V. H. Granville. Len. - St. Malo. PATty. - Roscoff. Sir. - EP. à Perkiridic. J. С. - Le Conquet. Langeron. - Laber-Ildut, Argenton. CrN. - Pen-Tir (Crozon). J. Ch. - Belle-Isle. Herb. J.B. de Bruxelles. - Le Croisic. Fu. D. - Le Ferrol. Col.

A. angustifolium Crn. - Z. I. Brest, le Mingant. CRN.

Pt. angustissimum (Griff.) J. Ag.

= Delesseria Griff. - Delesseria alata angustissima Ag. Hypoglossum Ktz.

Epiphyte de Laminaria digitata. - Jersey. Melle WHITE. Porspoder, Argenton. CRN.

\section{BONNEMAISONIÉES}

Bonnemaisonia.

\section{Bonnemaisonia asparagoides (Wood.) Ag.}

= Plocamium Lamour. - Ceramium Roth. - B. pilularia Ag.

EP. à Cherbourg. LE JoL. - Iles anglo-normandes. H. V. H. Roscoff, dragué au large de Duon. J. CH. - Banc de St. Marc, baies du Moulin-Blanc et de Bertheaume, Z. I. (ne découvrant pas) et EP. CRN. - Belle-Isle. Jouan. - EP. à la grande côte (Croisic). D. - EP. 
et pièges à Homards, Guéthary; Z. I. Gijon. S. - Abondant en juillet dans ces localités, presque disparu en septembre. J. CH.

Bonnemaisonia hamifera Hariot.

Dans la Manche et à Cherbourg. LE JoL.

\section{RHODOMÉLÉES}

Bostrychia.

Rhodomela.

Laurencia.

Halopithys.

Chondria.

Polysiphonia.

Pterosiphonia.

Brongniartella.

Dasya.
Heterosiphonia.

Ctenosiphonia.

Herposiphonia.

Janczewskia.

Lophosiphonia.

Ophidocladus.

Rytiphlœa.

Vidalia.

\section{Bostrychia scorpioides (Gmel.) Mont.}

= Rhodomela Ag. - Alsidium J. Ag. - Plocamium amphibium Lamour.

EP. à Nieuport. W. - Baie d'Authie (Berk-sur-mer). Gonse. Fécamp, St. Vaast, Granville. Chauv. - Réville. Le JoL. - Iles anglo-normandes. H. V. H. - Roscoff. Sir. - Arrière-port du Conquet. Langeron. - Penfeld, prés salés de Landerneau. CrN. Parmi les Salicornia des marais salants, au Croisic. FL. - La Teste-de-Buch. Chantelat. - Biarritz. Endress. - San Vicente, havres du Peral et de Villegas ; Rivadeo, Villavieja. S.

\section{Rhodomela brachygonia Cirn.}

Brest. CrN.

\section{Rh. lycopodioides (L.) Ag.}

= Rhod. subfusca Ag. - Rhod. gracilis Harv. - Rhod. tenuis. sima Kjellm. - Plusieurs auteurs, notamment Hauck p. 217, considèrent comme distincts le $R h$. lycopodioides (L.) Ag. et le $R h$. subfusca (Wood.) Ag. Mais de Toni ne voit qu'une seule espèce. 
Wimereux, Pointe-aux-Oies et Croy ; Roche-Bernard (Boulogne). Giard. - Dieppe. Leturquier. - Fécamp, Yport. D. - Luc. Chauv. - Cricqueville. Bertot. - Z. I. Cherbourg. Le Jol. - Iles anglo-normandes (les deux $R h$. de Hauck) H. V. H. - Roscoff. Sir. - EP. à Perkiridic. J. CH. - Rade de Brest, dragué ; EP. banc de St. Marc; Z. I. Laninon, Ste-Anne. CrN. - Le Croisic. Fu.

A. firmior J. Ag. - Iles anglo-normandes. H. V. H.

\section{Laurencia cæspitosa Lamour.}

$=L$. corymbifera Ktz.

Rivages atlantiques de France jusqu'au Calvados. Lamouroux. A rechercher.

\section{L. hybrida (D. C.) Len.}

$=$ Chondria Chauv. - L. caespitosa, L. angusta, L. pinnatifida cylindrica Harv. - L. cylindrica Ktz.

Fécamp. D. - Etretat, Antifer, St. Jouin. Bernard. - Arromanches. D. - Luc. Chauv. - Plages Napoléon et des bains, Flamands, Z. I. LE JoL. - Iles anglo-normandes. H. V. H. - St. Marc, Laninon, Ste.Anne. Crn. - Morbihan. Herb. J. B. de Bruxelles. Le Croisic. FL. - Biarritz, Guéthary, St. Jean de Luz. S. - La Corogne. Col.

Forme naine. - Z. S. Cherbourg. LE JoL.

\section{L. obtusa (Huds.) Lamour.}

$=$ Chondria Ag. - L. lutea Lamour.

St. Valéry-en-Caux. Leturquier. - Luc. D. - Cricqueville, Maisy. Bertot. - Grandcamp. D. - Z. M. Hommet, Querqueville, Flamands. Le JoL. - Iles anglo-normandes. H. V. H. - St. Malo. Melle Lebrun. - Roscoff. J. Ch. - Le Conquet. Langeron. - Banc de St. Marc, EP. Crn. - Morbihan. Prouhet. - Le Croisic. Fl. Biarritz, Guéthary, St. Jean de Luz, San Vicente, Gijon, Rivadeo, la Corogne. S.

A. paniculata Ktz. (=L. paniculata Ktz. - De Toni rapporte cette forme à $L$. pinnatifida). - Un échantillon douteux a été récolté à Jersey ; voir Van Heurck.

B. patentirama. - Roscoff, roche du Loup. Melles V. et K.

C. pyramidata J. Ag. (=L. pyramidalis Ktz.) - Z. I. Cherbourg. Le Jol. - Granville. Len. - Roscoff. Sir. - Pointe de l'île 
de Bas. Melles V. et K. - Z. I. Ste-Anne, Bertheaume. Crn. Jetée du Croisic. D. - La Rochelle. HerB. Zan.

Forme très réduite (3 à 4 millimètres) en épais gazon sur roche, cap du Figuier. J. Сн.

\section{Laurencia pinnatifida (Gm.) Lamour.}

= Chondria Ag.

EP. à Blankenberghe et à Ostende. W. - Les trois zones du Gris-Nez au Portel et sur tout le littoral de la Seine infre. D. Calvados, Cherbourg, Granville. Chauv. - Iles anglo-normandes. H. V. H. - Roscoff. Sir. - Z. I. le Conquet. Langeron. - Z. M. environs de Brest. CRN. - Lorient. Herb. ZAN. - Le Croisic. FL. D. - Z. I. Biarritz, Guéthary, St. Jean de Luz, San Vicente, Gijon, Rivadeo ; EP. à Rivadeo, exemplaires atteignant 20 ctm.; la Corogne. S. - Le Ferrol. CoL.

৯ A. cartilaginea (= Gelidium pinnatifidum Lyngb.) - Côtes atlantiques d'Europe. J. AG. A rechercher.

B. Osmunda (Gmel.) Ktz. - Cherbourg. Len. - Ste-Anne, le Minou. Cre. - Biarritz. Endress.

Forme naine. - Z.S. Cherbourg. Le JoL.

\section{Halopithys pinastroides (Gmel.) Ktz.}

= Rytiphloea, Rhodomela Ag. - Rhodomela pinastroides episcopalis, Rhod. episcopalis Mont.

Grandcamp. D. - Côtes du Calvados, Z. M. Cherbourg. Chauv. - Iles anglo-normandes. H. V. H. - Granville. Herb. Zar. St. Malo. Herb. J. B. de Bruxelles. - Z. I. rade de Brest, dragué. Environs de Brest. CRN. - Camaret, au pied de la falaise, à l'E. du port. J. CH. - Lorient. Herb. ZaN. - Z. I. le Croisic. FL. D. - EP. la Teste-de-Buch. Chantelat. - Biarritz, Guéthary, St. Jean de Luz. S. - La Corogne. Laz.

\section{Ghondria cærulescens (Crn.) Falk.}

$=$ Chondriopsis J. Ag. - Laurencia Crn. - Laurencia dasyphylla Lloyd. Ardissone.

Z. M. Langrune. D. - St. Vaast. Le JoL. - Z. I. entrée du port de Brest, Camfrout, passage de Plougastel. CRN. - Port et chenal du Croisic. Fu. - La Govelle. D. - Biarritz, Guéthary, 
St. Jean de Luz, San Vicente, Rivadeo; de petite taille. La Corogne. S.

Cette espèce dégage à l'état frais et pendant qu'elle sèche une forte odeur de triméthylamine.

\section{Ghondria dasyphylla (Wood.) Ag}

\section{= Chondriopsis J. Ag. - Laurencia Grev.}

Wimereux, Pointe-aux-Oies et Croy; la Crèche, St. Valéry-enCaux, Petites-Dalles, Fécamp, Yport. D. - Antifer. Bernard. Quihot, Langrune, Arromanches, Port-en-Bessin, Grandcamp. D. Côtes du Calvados. Len. - Z. I. Cherbourg. Le JoL. - Iles anglonormandes. H. V. H. - Roscoff. Sir. - Duon. J. CH. - EP. au Conquet. Langeron. - Z. I. sous le Château de Brest, St. Marc, anse du Minou. CRN. - Le Croisic. Fl. - Grande côte (Croisic). D. Biarritz, Guéthary, la Goureppe, Gijon, Rivadeo; à la Corogne, abondant et de grande taille. S.

A. articulata Ag. - Brest. CrN.

Ch. tenuissima (Good. et Wood.) Ag.

= Chondriopsis J.Ag. - Laurencia Grev. - Gigartina Lamour. - Laurencia dasyphylla squarrosa Harv.

Z. M. et Z. I. Quihot, Langrune, Grandeamp. D. - Côtes du Calvados. Len. - Luc, Arromanches, Port-en-Bessin. Chauv. - Z. I. Cherbourg. Le Jox: - Iles anglo-normandes. H. V. H. - Roscoff. Sir. - Ruisseaux de la plage coulant à marée basse, Perkiridic. J. Ch. - Le Conquet. Langeron. - Z. I. Laninon. Crn. - Plage de St. Goustan (Croisic). FL. - Grande côte, jetée du Croisic. D. Gijon. S.

Polysiphonia atro-rubescens (Dillw.) Grev.

$=$ P. discolor Ktz. - Hutchinsia Agardhiana Ag.

EP. à Nieuport. Kx. - Audresselles; Wimereux, Pointe-auxOies; la Crèche, le Portel, Petites-Dalles, Fécamp, Yport. D. Antifer. Bernard. - Le Havre. Hepp. - Luc, Arromanches. Chauv. - Z. I. Cherbourg. Le JoL. - Iles anglo-normandes. H. V. H. Roscoff. Sir. Melles V. et K. - Sous le Château de Brest, St. Marc, Laninon. Crn. - Au pied de la falaise de Toulinguet (Crozon). J. Ch. - Morbihan. Leuièvre. - Le Croisic. D. - Gijon, Rivadeo, la Corogne. S. - Le Ferrol. CoL. 
A. Deschampsii Crn. (== P. Agardhiana Grev.) - Cherbourg. Le Jol. - Jersey 1900. H. V. H. - Finistère. CRN.

\section{Polysiphonia Brodiæi (Dillw.) Grev.}

\section{$=$ Hutchinsia penicillata $\mathrm{Crn}$.}

Rochers du Calvados. Chauv. - Z. I. Querqueville, Digue; bouées et bois de l'avant-port militaire. LE JoL. - Iles anglo-normandes. H. V. H. - Roscoff. Sir. - Z. I. entrée du port de Brest, Kervallon, le Minou. CRN. - Chenal du Croisic. Fu. - Grande côte (Croisic). D. - La Corogne. S.

A. angustissima H. V. H. - Jersey 1900. H. V. H.

B. densa Holm. et Batt. - Iles anglo-normandes, avec le type. BatT.

Forme pénicillée Crn. - Chenal de la rade de Brest, dragué ; banc de St. Mare, EP. CRN.

P. cæspitosa Thur.

Biarritz. Lespinasse.

\section{P. collabens $\mathrm{Ktz}$.}

\section{= Streblocladia (Ag.) Falk. - Hutchinsia Ag.}

Biarritz. Thur. - Z. I. Biarritz, Guéthary, St. Jean de Luz, Gijon, Rivadeo, la Corogne. S.

\section{P. elongata (Huds.) Harv.}

Z. M. et Z. I. Wimereux, Pointe-aux-Oies, Croy et la Tablette; Senneville, Fécamp, Yport. D. - Bénouville. Bernard. - Etretat. D. - Le Tilleul, Antifer. Bernard. - Quihot, Langrune, Arromanches, Port-en-Bessin, Grandcamp. D. - Côtes du Calvados. Len. - Z. I. Cherbourg. Le JoL. - Iles anglo-normandes. H. V. H. Roscoff. Sir. - Environs de Brest. CRN. - Le Croisic. FL. - Chenal du Pouliguen, la Govelle, jetée du Croisic. D. - La Rochelle. Herb. Zan. - La Teste-de-Buch. Chantelat. - San Vicente, Gijon. S.

A. denudata J. Ag. - Portbail (Manche). Herb. J. B. de Bruxelles. - Banc de St. Marc, EP. CrN.

B. rosea J. Ag. - Banc du Moulin-Blanc. CRN. 


\section{Polysiphonia elongella Harv.}

Z. I. au-dessous des rochers de Longlet et à l'entrée du port militaire. Le JoL. - Iles anglo-normandes. H. V. H. - Banc du Moulin-Blanc et dragué, rade de Brest, sur Cystoseira discors. CRN.

\section{P. fastigiata (Roth.) Grev.}

Littoral belge, EP. Kx. - Ostende et Nieuport. MaC.Leod. Gris-Nez. D. - Dieppe. Herb. ZaN. - EP. à St. Jouin. Bernard. Toujours sur Ascophyllum, Cherbourg. Chauv. - Iles anglo-normandes. H. V. H. - Granville. Herb. Zan. - Roscoff. Sir. Environs de Brest. CRN. - Sur Ascophyllum et Fucus, au Croisic. Fu. D. - EP. à Guéthary, San Vicente, Rivadeo. S. - Le Ferrol. Col.

\section{P. ferulacea Suhr.}

Biarritz, Guéthary, St. Jean de Luz, San Vicente, Rivadeo. S.

P. fibrata (Dillw.) Harv.

Sur bancs de Moules à Blankenberghe. Kx. - Sur pilotis de l'estacade W. à Ostende. W. - Grandcamp. D. - Cherbourg. LE JoL. - Iles anglo-normandes. H. V. H. - Roscoff. Sir. Bertheaume, pointe de Corson. CRN.

P. fibrillosa Grev.

$=P$. violacea Kleen.

Bancs d'huîtres à la Panne (fixé ?) Kx. (Herb. J. B. de Bruxelles sous le nom de P. violacea fibrillosa). - Fécamp, Yport. D. Antifer. Bernard. - Entre Lion et Luc. Bertot. - Grandcamp. D. - Cherbourg. Le Jol. - Mles anglo-normandes. H. V. H. - Roscoff. Sir. - Le Conquet, EP. Langeron. - Banc de St. Mare, Laninon. CRN. - Sur Chorda filum, au Croisic. FL. D. - Rivadeo, sur Codium tomentosum. S. - San Vicente, la Corogne. Laz.

\section{P. flexella J. Ag.}

= Hutchinsia tingitana Schousb. - Dasya Solierii J. Ag. Golfe de Gascogne. Born. - Bayonne. Blytт.

P. fœtidissima Cockx.

$=P$. intricata crassa Ardiss.

St. Vaast. Le Jol. - Biarritz. Thur. - Biarritz, Guéthary, St. Jean de Luz. S. 
Polysiphonia fruticulosa (Wulf.) Spreng. tula Ktz.

$=P$. Wulfenii J. Ag. - P. Martensiana, P. humilis, P. coma-

Luc. BеRтот. - Grandcamp. D. - Granville. Len. - Cherbourg, Z. M. LE JoL. - Iles anglo-normandes. H. V. H. - Roscoff. Sir. Laninon, St. Marc. CrN. - Le Croisic. D. - Mares au Croisic. Fl. - La Teste-de-Buch. Chantelat. - Fond du golfe de Gascogne. S. -- La Goureppe. J. Сн. - Biarritz. Endress. Postels. - Biarritz, Guéthary, St. Jean de Luz. Lespinasse. - San Vicente, Gijon, Rivadeo, la Corogne. S.

A. glomerata Suhr. - Biarritz. Endress.

B. tenuissima H. V. H. - Jersey 1902. H. V. H.

P. furcellata (Ag.) Harv.

$=P$. ceramiiformis Crn. $-P$. forcipata J. Ag. $-P$. laevigata Ktz.

Côtes de France. Bonnem. Chauv. - St. Vaast-la-Hougue. LE JoL. - Iles anglo-normandes. H. V. H. - Sous le Château, Laninon. CRN.

A. patula J. Ag. - Banc de St. Mare, EP. Crs.

\section{P. Guernisaci (Crn.) J. Ag.}

= Dasya Crn.

Z. I. Brest, bane de St. Marc. CRN.

\section{P. hermaphrodita Näg.}

Cherbourg. Liste supplémentaire manuscrite, LE JoL.

P. insidiosa Crn.

$=P$. havanensis insidiosa J. Ag.

Attaché aux pierres à Ostende. Kx. - Z. M. rochers de Longlet, St. Vaast. Le JoL. - Z. I. St. Marc, Laninon, banc du Moulin-Blanc. CRN.

\section{P. insignis Crn.}

Rade de Brest, dragué. CRN. 
Polysiphonia intricata J. Ag.

$=$ Lophosiphonia subadunca (Ktz.) Falk. var. intricata J. Ag. - P. uncinata Ktz. - P. subadunca intricata Hauck.

Z. S. Camfrout. CRN.

\section{P. macrocarpa Harv.}

$=P$. sertularioides (Grat.) J. Ag. - P. pulvinata Harv. -$P$. subtilis Ardiss. - D'après Batt. $=P$. sertularioides Holm. et Batt. + P.pulvinata Phyc. Br.

Wimereux, la Rochette et Croy; la Crèche. D. - Etretat. Bernard. - Iles anglo-normandes. H. V. H. - Z. I. rade de Brest. CRN. - Au pied de la falaise de Toulinguet (Crozon). Dét. Reinbold. J. Сн. - Port-Vieux de Biarritz, au pied de la falaise ; San Vicente, Rivadeo. S.

\section{P. multifida Duby.}

Le Ferrol. CoL.

P. multifida Duby, m'écrit De Toni, paraît la même chose que $P$. foeniculacea (Drap.) J. Ag. Mais P. multifida Ktz. = P. polychotoma Ktz. Sp. Alg. = P. Brodiaei (Dillw.) Grev. Il faudrait revoir ces espèces sur échantillons authentiques avant de se prononcer d'une façon définitive.

\section{P. nigrescens (Dillw.) Grev.}

$=P$. violascens $\mathrm{Ktz} .-P$. secundata, Hutchinsia senticosa Suhr. - Hutchinsia Ag. - P. fucoides Grev.

Sur les pierres des brise-lames à Ostende. W. - Fossé d'une huîtrière à Ostende. Mac-Leod. - EP. sur tout le littoral belge; chenal de Nieuport sur Buccins. Kx. - Blanc-Nez, du Gris-Nez au Portel, et tout le littoral de la Seine infre. et du Calvados. D. Cherbourg, Granville. Chauv. - Iles anglo-normandes. H. V. H. Roscoff. Sir. - Toulinguet (Crozon). Dét. Reinbold. J. CH. - Chenal du Croisic. Fl. D. - Morbihan. Lelièvre. - La Rochelle. Herb. Zan. - Pointe d'Aiguillon (Teste-de-Buch). Chantelat. - San Vicente, Gijon, Rivadeo. S.

A. affinis Harv. - Z. I. au-dessous des rochers de Longlet. LE JoL. - Iles anglo-normandes. H. V. H. - Rade de Brest, dragué. CRn.

B. congesta Len. - Port, Arromanches. Len.

C. delicatissima H. V. H. - Jersey 1900. H. V. H. 
D. fucoides J. Ag. - Côtes de la Manche. BréB. - Iles anglonormandes. BAтt. - Environs de Brest. CrN.

E. pectinata J. Ag. - Iles anglo-normandes. BATT. - Bancs de St. Marc et du Moulin-Blanc. CrN.

Forme capillaire (de 2 à $3 \mathrm{ctm}$.) - Arromanches. Herb. J. B. de Bruxelles.

\section{Polysiphonia opaca (Ag.) Zan.}

Iles anglo-normandes H. V. H. - Biarritz, Guéthary, St. Jean de Luz, Rivadeo. S.

A. ascendens J. Ag. ( $=$ P. ascendens Crn. $)-$ Z. M. Ste-Anne, Bertheaume. CRN.

$$
\text { P. polyspora J. Ag. }
$$

Biarritz. Thur. Lespinasse. - Biarritz, Guéthary, St. Jean de Luz, San Vicente. S.

\section{P. pulvinata J. Ag.}

$=$ Hutchinsia Ag. - P. arenaria Ktz.

En eau saumâtre, sur pierres du canal Bruges-Ostende. MaCLeod. - Hommet, Querqueville, Nacqueville. LE Jol. - Belle-Isle. Lroyd. - Le Croisic. Fu. - Grande côte (Croisic). D.

\section{P. scopulorum Harv.}

Bayonne, Biarritz. Born. FL.

Espèce australienne.

$$
\text { P. sertularioides (Grat.) J. Ag. }
$$

$=$ P. macrocarpa, P. pulvinata Harv. - P. bellula, P. decipiens, $P$. tenella. De Not.

Anses du Minou et de Déalbors. CRN. - Roches blanches à la Goureppe. Dét. Reinbold. J. Сн.

\section{P. simulans Harv.}

$=P$. spinulosa Harv.

St. Vaast. Herb. ZaN, - Z. 1. Hommet, Querqueville. Le Jol. Iles anglo-normandes. H. V. H. - Banc de St. Marc. CRN. 


\section{Polysiphonia spinosa (Ag.) J. Ag.}

$=$ P. spinulosa Grev. - Hutchinsia Ag. - P. fibrillosa J. Ag.

A. major J. Ag. (= P. Carmichaeliana Harv.) - Iles anglonormandes. H. V. H. - Environs de Brest. CRN. - San Vicente de la Barquera. LAz.

Carmichael demande si cette forme méditerranéenne est identique à celle des côtes d'Ecosse.

\section{P. subcontinua (Ag.) J. Ag.}

Cherbourg. Herb. J. B. de Bruxelles.

Espèce de la Méditerranée, signalée à Cadix par CABrera. Douteuse à Cherbourg.

\section{P. subulifera (Ag.) Harv.}

= Hutchinsia Ag. - P. armata J. Ag.

Iles anglo-normandes. H. V. H. - Bancs de St. Marc, Laninon. Crn. - Belle-Isle. Lloyd.

\section{P. thuyoides Harv.}

$=$ Rytiphlœa Harv. - P. rigidula Bonnem.

Sur pierres du port d'Ostende, recevant l'eau douce à basse mer. W. Kx. - EP. sur grandes Algues à Nieuport. Kx. - Z. M. Cherbourg. LE JoL. - Iles anglo-normandes. H. V. H. - Roscoff. Sir. - Duon. Melles V. et K. - Z. I. anse du Minou, Bertheaume, pointe de Corson. Crn. - Belle-Isle. Desmaz. - La Govelle (Croisic). D. - San Vicente, la Corogne. S.

A. patens Hook. - EP. entre Ostende et Nieuport. Kx.

\section{P. urceolata (Lightf.) Grev.}

$=P$. denticulata Ktz. $-P$. formosa Suhr. $-P$. stricta Grev.

D'après M. Bornet, il faudrait réviser les stations du Polysiphonia urceolata, parce que beaucoup d'exemplaires de ce nom, même dans les grands herbiers, sont mal déterminés. De Toni pense qu'on a pu le confondre avec $P$. sertularioides.

Ostende sur pilotis (?) Herв. J. B. de Bruxelles. - Z. I. St.Jouin, Bruneval. Bernard. - Port-en-Bessin. D. - Cricqueville. Bertot. Z. M. et Z. I. Cherbourg. LE JoL. - Iles anglo-normandes. H. V. H. - St. Malo. Heri. J. B. de Bruxelles. - Z. I. sous le Château de Brest, Laninon. CrN. - Morbihan. Prouhet. - Le Croisic. Fu. D.

A. comosa J. Ag. (= P. stricta Grev.) - Z. S. Wimereux, Croy. D. - Yport, St. Jouin. Bernard. - Luc, Langrune. D. - St. Aubin, 
Arromanches. Chauv. -- Iles anglo-normandes. H. V. H. - Rade de Brest. Crn. Desmaz. - Au Pila (Teste-de-Buch.) Chantelat. EP. CRN.

B. formosa J. Ag. - Baies du Moulin-Blanc et de Bertheaume,

C. patens J. Ag. - Jersey 1903. H. V. H. - Environs de Brest. CRn.

D. roseola J Ag. - Baie du Moulin-Blane, Laninon. CRN.

Forme capillaire (2 à $\mathbf{3} \mathrm{ctm}$.) - Côtes du Calvados. Len.

Polysiphonia variegata (Ag.) Zan.

$=$ P. denudata Ktz. - P. Biasolettiana J. Ag. - Hutchinsia Ag.

St. Vaast. Le Jol. - Iles anglo-normandes. H. V.H. - Entrée du port de Brest. Crn. - Morbihan. Prouhet. - Chenal et port du Croisic. FL. - Chenal et port du Pouliguen. D.

\section{P. violacea (Roth.) Grev.}

D'APres De ToN $=P$. subulata Crn. $-P$. aculeata ot $P$. aculeifera $\mathrm{Ktz}$.

$\mathrm{Ne}$ descend pas au sud du Kattegat. Les échantillons de ce nom provenant de la Méditerranée appartiennent au P. subulata (Ducl.) Hauck.

Formes : robusta et allochroa.

Var : subulata (Ducl.) Hauck. $=$ P. subulata J. Ag. - P. Montagnei De Not. - P. Perreymondii J. Ag. - P. Griffithsiana Harv.

Méditerranée et Atlantique jusque sur les côtes S. d'Angleterre.

Formes : Montagnei et Perreymondii.

D'APRÈs Crouan, trois formes : robusta J. Ag. (= P. subulata Bonnem. Crn.) - Laninon, St. Marc, Laber-Ildut.

allochroa J. Ag. (= P. allochroa Ktz. $-P$. violacea Harv.) Laninon, Laber-Ildut.

violacea J. Ag. (= P. violacea Crn. Exsicc. 297. - Grammita subulata major Bonnem.) - Embouchure des rivières marines, bancs du Moulin-Blanc et de St. Marc.

Et en outre, comme espèce :

P. subulata (Ducluz.) J. Ag. (= Ceramium subulatum Ducluz.) var. Montagnei (=P. Montagnei De Not.) - Sur les Corallines, baie de Bertheaume. 
D'APRÈs HAUCK (p. 225) = Ceramium Roth. - P. violacea Grev.

genuina (= P. violacea J. Ag. - P. angulosa Ktz.) - Mer du Nord, Atlantique et Adriatique.

subulata (= P. subulata Ducluz. J. Ag. - P. Perreymondii J. Ag. - P. Montagnei De Not.) - Adriatique.

tenuissima $(=P$. violacea tenuissima Aresch. $-P$. roseola, P. divaricata Ktz.) - Atlantique.

Il est impossible de concilier les auteurs ; il faudrait une étude nouvelle de ces différentes formes.

Je trouve en outre les stations suivantes :

P. subulata. - EP. à Nieuport. Kx. - Ostende. Dasman. Cherbourg. Len. - Iles anglo-normandes. Batt. - Gijon. Durieu.

P. allochroa. - Sur antenne de Homard à Ostende. Herb. J. B. de Bruxelles.

P. Griffithsiana. - Jersey. Melle White. - San Vicente de la Barquera. Laz.

P. peucedanoides Crn. (=P. violacea $)$. - Laninon. CRN.

P. violacea Duby. - Pointe d'Aiguillon (Teste-de-Buch). Chantelat.

\section{Pterosiphonia complanata (Clem.) Falk.}

= Polysiphonia J. Ag. - Rytiphloea Ag. - Odonthalia Crn. - Rhodomela cristata Duby. - Polysiphonia cristata Harv. Plocamium cristatum Lamour.

Roscoff, roche du Loup, Duon. J. Ch. - Z. I. anses de Ste-Anne et du Minou. Crn. - Biarritz, Guéthary, St. Jean de Luz, San Vicente, Gijon, Rivadeo, la Corogne. S. - Portugalete, le Ferrol. Col. - Comillas, Candas. Laz.

Pt. parasitica (Huds.) Falk.

$=$ Polysiphonia Grev. - Hutchinsia Ag.

Calvados. Duby. - Roscoff. Melles V. et K. - Brest, baie de Laninon. CRN. - Biarritz. S.

Pt. pennata (Roth.) Falk.

= Hutchinsia Ag. - Polysiphonia J. Ag. - Polysiphonia pinnulata Ktz. - Ceramium Roth.

Côtes du Calvados. Len. - Z. I. Yport, Arromanches. D. - 
Port-en-Bessin. Chauv. - Granville. Bréb. - Z. I. anse du Minou, Bertheaume. CrN. - Z. M. grande côte (Croisic). D. - Z. I. Biarritz, épave à Guéthary, St. Jean de Luz, San Vicente, Gijon. S.

Brongniartella byssoides (Good. et Wood.) Schm.

$=$ Hutchinsia Ag. - Polysiphonia Grev.

Côtes du Calvados. Len. - Z. I. Hommet, Querqueville, Nacque. ville; souvent EP. LE JoL. - Iles anglo-normandes. H. V. H. Roscoff. Sir. - Laninon, le Douric, Bertheaume. CrN. - Grande côte (Croisic). D. - Cap Ferret (Teste-de-Buch). Chantelat.

A. denudata. - Cherbourg. Duvernox. - Portbail. Herb. J. B. de Bruxelles.

Dasya arbuscula (Dillw.) Ag.

$=$ D. Hutchinsiae Harv. - D. spinella Crn. - Callithamnion Lyngb.

Dieppe, St. Vaast, Granville. Herb. J. B. de Bruxelles. - Arromanches. Chauv. - Cherbourg. Duvernoy. - Z. I. Hommet, Querqueville, Nacqueville. Le JoL, - Iles anglo-normandes. H. V.H. - Roscoff. Sir. - Brest. HeRB. J. B. de Bruxelles. - Biarritz. Postels. - Andagorria. J. Сн. - Guéthary, San Vicente, Rivadeo. S. - La Corogne. Col.

A. caspitosa J. Ag. - Banc du Château, le Délec. Crn.

B. ceratophylloides J. Ag. - Bane de St. Marc. Cre.

C. subarticulata J. Ag. - Bertheaume. CRN.

\section{Cattlowiæ Harv.}

Batters la réunit à $D$. punicea Menegh.

Jersey. Échantillon unique découvert en 1858 près de St. Elisabeth Castle par Melle Mary Cattrow.

D. corymbifera J. Ag.

$=D$. venusta Harv. - D. arbuscula mucilaginosa Crn

Attainville, Carteret. Herb. ZAN. - Iles anglo-normandes.H.V.H. - Rade de Brest, dragué. Banc de St. Marc, EP. CRN.

D. ocellata (Grat.) Harv.

= Ceramium Grat. - Hutchinsia Ag. - D. simpliciuscula Ag. Dragué, rade de Brest, baie du Moulin-Blanc. CRN. - Le Croisic Fu. - Le Ferrol. CoL. 


\section{Dasya punicea Menegh.}

Côtes méridionales de l'Angleterre. Batr. Holmes, J. AG. Merrifield. De Toni demande si l'on n'a pas pris pour tel le $D$. venusta Harv.

\section{D. spinella Ag.}

$=$ Dasyopsis (Ag.) Zan.

Portbail. Herb. J. B. de Bruxelles.

C'est une espèce de la Méditerranée et de l'Adriatique.

\section{D. spinella Duby.}

= Wrangelia penicillata Ag. - Griffithsia penicillata Ag. Ceramium Boucherii Duby.

Côtes atlantiques de l'Europe mérle. De Toni. - Iles Chausey. BrÉb. in Desmaz. - Brest, banc du Château. Desmaz.

A. mucilaginosa (= Cer. Boucherii mucilaginosum Crn. Cer. ocellatum Grat.) - Banc de St. Mare. Desmaz.

B. tenuissima (= Cer. Boucherii tenuissimum Crn.) - Banc de St. Marc. Desmaz.

D. verticillata Harv.

$=$ Lophothalia Ktz.

EP. à Nieuport. Kx.

Espèce de Tasmanie.

Heterosiphonia coccinea (Huds.) Falk.

$=$ Dasya Ag.

EP. à Ostende et à Nieuport. W. - Z. I. et souvent EP. à Cherbourg. Le JoL. - Iles anglo-normandes. H. V. H. - Roscoff. Sir. - EP. à Ouessant; Z. I. au Conquet. Langeron. - Le Croisic. FL. D. - Biarritz, Guéthary, St. Jean de Luz; Z. I. San Vicente, Gijon, Rivadeo; la Corogne. S. - Santander, Comillas, Llanes, Candas. LAz. - Portugalete. CoL.

A. crassa J. Ag. - Atlantique. De Tonr. A rechercher.

B. hirsuta J. Ag. - EP. à Cayeux, Ault, Tréport ; Z. I. PetitesDalles, Fécamp, Yport, Etretat. D. - St. Jouin, Cauville. Bernard. - Luc, Arromanches, Port-en-Bessin, Grandcamp. D. - Environs de Brest. CRN.

C. patens (Grev.) J. Ag. - Rade de Brest, dragué. Crn. 
D. squarrosa Harv. - Quihot près de Luc. D.

E. tenuis J. Ag. - Anse du Minou. Cren.

Ctenosiphonia hypnoides (Welw.) Falk.

= Polysiphonia Welw.

Gijon. S.

Herposiphonia tenella (Ag.) Näg.

= Polysiphonia J. Ag. - Hutchinsia Ag.

Biarritz. Thur. Lespinasse. - Sur les Lithothamnion, Z. S. Biarritz, Guéthary, St. Jean de Luz, Rivadeo. S. - Andagorria. J. Сн.

\section{$\star$ Janczewskia verruciformis Solms.}

Sur Laurencia obtusa. A rechercher.

\section{Lophosiphonia obscura (Ag.) Falk.}

$=$ Polysiphonia J. Ag. - P. adunca, P. virens Ktz.

Z. M. Hommet. Le JoL. - Guernesey. Melle LeLièvre. - Jersey. Melle White. - Brest. Herb. J. B. de Bruxelles. - Au pied de la falaise de Toulinguet (Crozon). Dét. Reinbold. J. Cн. - Le Croisic. FL. - Biarritz, Port-Vieux, au pied de la falaise ; Guéthary, St. Jean de Luz, San Vicente, Gijon, Rivadeo, la Corogne. S.

\section{Ophidocladus Schousboei (Thur.) Falk.}

$=$ Polysiphonia Thur. - Donné par Schousboe sous cinq noms différents au moins.

Biarritz. S. Born. Lespinasse. - Plateau de la Chinaougue à Biarritz. THUR.

On ne connât qu'une seconde station de cette espèce (à Tanger).

O. simpliciusculus (Crn.) Falk.

= Polysiphonia Crn. - Polysiphonia obscura Harv. - Polysiphonia coralloides Suhr.

Z. I. anse du Minou, près du phare. CrN. - Biarritz. Thur. Cette station est probablement celle de la Goureppe, plus exactement la Roche-qui-boit. J. Сн. - La Goureppe (Guéthary), San Vicente, Gijon. S. 
Rytiphlœa tinctoria (Clem.) Ag.

R. rigidula Ktz. - R. semi.cristata J. Ag.

EP. à Ostende. Herb. J. B. de Bruxelles. - Laninon, St. Marc ; rade de Brest, dragué. CRN.

\section{Vidalia volubilis (L.) J. Ag.}

= Dictyomenia Grev. - Rhodomela Ag.

EP. à Ostende. Herb. W. - EP. à la Panne. Kx.

La station la plus proche connue est Tanger.

\section{CÉRAMIÉES}

Sphondylothamnion.

Spermothamnion.

Ptilothamnion.

Griffithsia.

Halurus.

Bornetia.

Monospora.

Pleonosporium.

Rhodochorton.

Callithamnion.
Phlebothamnion.

Seirospora.

Compsothamnion.

Plumaria.

Ptilota.

Antithamnion.

Crouania.

Spyridia.

Ceramium.

Microcladia.

Sphondylothainnion multifidum (Huds.) Näg.

= Callithamnion Ktz. - Wrangelia J. Ag. - Griffithsia Ag. - Corynospora Crn.

Z. I. Grandes-Dalles, Senneville, Luc, Langrune, Arromanches. Chauv. - Marigny. Bertot. - Grandcamp. D. - St. Vaast. Len. - Z. I. Cherbourg. Le JoL. - Iles anglo-normandes. H. V. H. St. Malo. Thur. - Roscoff. Sir. - Dragué au large de Duon ; filets des pêcheurs. J. Ch. - Le Conquet. Langeron. - Z. I. Brest. Herb. Zan. - Banc de St. Marc, Bertheaume. Crn. - Camaret. Dẹ́t. Reinbold. J. Сн. - Le Croisic. Fl. Thur. - Grande côte (Croisic). D. - Biarritz, Guéthary, St. Jean de Luz, San Vicente, Gijon. S.

A. piliferum Ag. - Arromanches. Herb. J. B. de Bruxelles. 
Spermothamnion barbatum (Ag.) Näg.

= Callithamnion Ag. - Call. Ralfsii Harv.

Z. I. passage de Plougastel, Bertheaume. Crv.

Sp. intricatum (Ag.) Näg.

= Callithamnion Ag. - Ceramium Ag. - Trailliella Batt. - Sp. Turnerii intricatum Holm. et Batt.

Grandcamp. D. - Commun à Gatteville sur roches et Algues; stérile. MaLard et Kuckuck. - Iles anglo-normandes. H. V. H. Brest. NäGeli. - Anse du Minou. CRN.

Sp. roseolum (Ag.) Pringsh.

$=$ Callithamnion Ag. - Call. repens Lyngb.

Sur Polyides, Luc. Herb. J. B. de Bruxelles. - Brest. Herb. ZaN.

Sp. sphæricum (Crn.) Näg.

= Callithamnion Crn.

Anse du Minou, sur Ahnfeltia. CRN.

Sp. strictum (Ag.) Ardiss.

= Callithamnion Ag. - Call. unilaterale Zan. - Call. semi. pennatum J. Ag. - Call. Crouanii Ktz.

Dragué, rade de Brest. CRn. - Biarritz. Postels.

Sp. Turnerii (Mert.) Aresch.

= Callithamnion Ag. - Corynospora Crn. - Ceramium Mert. - Callithamnion sub-verticillatum Zan.

EP. au cap Blanc-Nez. De Block. - Z. I. du Gris-Nez au Portel, Petites-Dalles, Fécamp, Yport, Antifer. D. - Luc, Langrune. Chauv. - Arromanches, Grandcamp. D. - Côtes du Calvados. Len. BréB. Desmaz. - Z. M. Cherbourg. Le Jol. - Iles anglo-normandes. H. V. H. - Roscoff. Sir. J. CH. - Brest. Herb. Zan. - Bertheaume, Laber-Ildut, Argenton. CRN. - Le Croisic. D. - La Teste-de-Buch. Chantelat. - Biarritz, Guéthary, St. Jean de Luz, San Vicente; Gijon, cap Torres, sur Ahnfeltia. S.
A. minimum Crn. - Rade de Brest. CRN.
B. repens Dillw. - Z. I. Cherbourg. Le JoL.
C. sub-verticillatum Len. - St. Vaast. LEN. 
D. variabile (Ag.) Ardiss. (= Callithamnion abbreviatum Ktz. - Griffithsia tenuissima Zan. - Callithamnion Ag.) - EP. littoral belge. Kx. - Ostende, sur Furcellaria lumbricalis. W. Z. I. Wimereux, le Portel. D. - St. Vaast. Herb. ZAN, - Cherbourg. Herb. J. B. de Bruxelles. - Carteret. Herb. ZaN. - EP. anse du Minou, plage de Brest. Desmaz.

\section{Ptilothamnion micropterum (Mont.) Born.}

= Callithamnion Ktz. - Pt. pluma micropterum Hauck Callith. pinastroides Reinsch.

EP. à Guéthary. S.

\section{Pt. pluma (Dillw.) Thur.}

$=$ Callithamnion Ag.

Sur Saccorhiza bulbosa, EP. à Ostende. W. - Sur Laminaria Cloustonii, Cherbourg. Le JoL. - Iles anglo-normandes. Jersey 1903. H. V. H. - Sur stipe de Laminaria Cloustonii, Roscoff. J. Сн. Le Délec, baie de Bertheaume, Argenton. CRN. - Le Croisic. FL. Thur. - EP. à San Vicente, la Corogne. S.

\section{Griffithsia barbata (Sm.) Ag.}

$=$ Ceramium Duby.

Côtes de France. Bonnem. Len. - Calvados, Finistère. Desmaz. - Plage des Flamands, St. Vaast. Le Jol. - Iles anglo-normandes. H. V. H. - Portbail. Herb. J. B. de Bruxelles. - Baie du MoulinBlanc, banc de St. Mare, Laninon. CRN.

\section{Gr. corallina (Lightf.) Ag.}

EP. à Nieuport. W. - Quihot, près de Luc; Grandcamp. D. Z. I. plage Napoléon etc. LE JoL. - Iles anglo-normandes. H. V. H. - Portbail. Herb. J. B. de Bruxelles. - Granville. Len. -- St. Malo. HerB. J. B. de Bruxelles. - Ile Callot. Melles V. et K. - Roscoff. Sir. - St. Marc, le Délec. Crn. - Pen-Tir (Crozon). J. Ch. - Le Croisic. Fu. D. - Bayonne. Ag. - Santander. CoL.

\section{Gr. devoniensis Harv.}

Luc. Chauv. - Cherbourg. Herb. Zan. - Mles anglo-normandes. H. V. H. - Portbail. HerB. J. B. de Bruxelles. - Z. I. Kervallon. CrN. - Port du Croisic. FL. 


\section{Griffithsia Schousboei Mont.}

$=$ Gr. Opuntia J. Ag. - Gr. Giraudii Solier. Guéthary. S.

Gr. setacea (Ell.) Ag.

EP. à Ostende. Landszweert. - EP. à Nieuport. W. - Z. I. Gris-Nez, Audresselles, Ambleteuse, Wimereux, Boulogne, Ault, le Tréport, Dieppe, St. Valéry-en-Caux, Petites-Dalles, Fécamp, Yport, Etretat. D. - Le Tilleul, St. Jouin. Bernard. - Luc, Porten-Bessin, Grandcamp. D. - Cherbourg. LE Jol. - Iles anglonormandes. H. V. H. - St. Malo. Herb. J. B. de Bruxelles. Roscoff. Sir. - Chenal de l'île Verte. J. CH. - Bancs du Château, St. Marc, Laninon. CRN. - Le Croisic. Fu. D. - Gijon? la Corogne? S.

Halurus equisetifolius (Lightf.) Ktz.

$=$ Griffithsia Ag. $-H$. compactus Ktz.

EP. Wimereux, le Portel. D. - Z. I. Mesnilval, Fécamp, Yport, Etretat. D. - St. Jouin. Bernard. - Ste-Adresse. Dupray. - Luc, Arromanches, Grandcamp. D. - Z Z. I. Cherbourg. LE JoL. - Iles anglo-normandes. H. V. H. - Roscoff. Sir. - Z. I. le Minou, Bertheaume, pointe de Corson. CrN. - Camaret. J. Ch. - Le Croisic. Fu. - EP. à la grande côte (Croisic). D. - Cap Ferret (Teste-de-Buch). Chantelat. - Biarritz, Guéthary, St. Jean de Luz, San Vicente, Gijon, Rivadeo, la Corogne. S.

\section{H. simplicifilum (D. C.) Ktz.} J. Ag.

$=H$. equisetifolius simplicifilum, Griffithsia simplicifilum

Iles anglo-normandes. H. V. H. - Baie de Bertheaume, EP. CrN. - Côtes atlantiques de France. Len. Gatllon. Bonnem.

\section{Bornetia secundiflora (J. Ag.) Thur.}

= Griffithsia cymiflora, Griff. ligustica Ktz. - Griffithsia J. Ag. - Griff. corallina (pro parte) Ag. Bonnem. Desmaz.

Z. I. Cherbourg; ne fructifie pas. LE JoL. - Iles anglo-normandes. H. V. H. - Roscoff. Sir. - Dragué à Duon. J. CH. - Z. I. Laninon, banc du Château, Ste-Anne. CRN. - Biarritz, Guéthary, St. Jean de Luz; fructifie. S. Lespinasse. - San Vicente, Gijon, la Corogne. S. 
Monospora pedicellata (Sm.) Sol.

= Callithamnion Ag. - Ceramium Duby. - Corynospora J. Ag. - Mon. clavata J. Ag. - Mon. pedicellata clavata Zan.

Quihot, Arromanches, Port-en-Bessin, Grandcamp. D. - Z. I. Cherbourg. Le Jou. - Iles anglo-normandes. H V. H. - St. Malo. Herb. J. B. de Bruxelles. - Roscoff. Sir. - Autour de la roche du Loup. J. Сн. - Banes du Moulin-Blane et de St. Mare; Kervallon. CrN. - Morbihan. Prounet. - Chenal du Croisic. Fl. - Biarritz, Guéthary, St. Jean de Luz, San Vicente, Gijon, Rivadeo, la Corogne. S.

De Toni réunit sous le nom de Mon. pedicellata les formes Mon. clavata de divers auteurs. - Les deux espèces de Monospora sont reliées par tous les intermédiaires (Bornet). - Batters indique comme espèce distincte : Mon. clavata J. Ag. Localité : Aurigny.

Pleonosporium Borrerii (Sm.) Näg.

= Callithamnion Harv. - Call. Vidovichii Menegh. - Call. seminudum Ag.

Z. I. Petites-Dalles, Fécamp. D. - Bruneval, St. Jouin. Bernard. - Luc, Arromanches. Chauv. - Côtes du Calvados. Len. - Z. I. Hommet, Querqueville. Le JoL. - Iles anglo-normandes. H. V. H. - Banc du Château à Brest. CRN. - Toulinguet (Crozon) J. CH. Le Croisic. Fu. - La Goureppe (Guéthary), Rivadeo, la Corogne. S.

A. flabellatum Ktz. - Arromanches. Len. - Guéthary. J. Сн.

Pl. flexuosum (Ag.) Born.

= Callithamnion flexuosum, Call. pulchellum Ag.

Z. I. Biarritz [qui est sa limite septle]; San Vicente, Rivadeo. S.

P1. pinnatum (Crn.) J. Ag.

= Halothamnion, Corynospora J. Ag. - Griffithsia Crn.

Bane du Moulin-Blanc. CRN.

\section{Rhodochorton Brebneri Batt.}

Sur Gloiosiphonia capillaris. Plymouth. Batт.

Rh. floridulum (Dillw.) Thur.

= Callithamnion Ag. - Thamnidium Thur.

Z. M. et Z. S. Audresselles; Wimereux, la Rochette et Croy; tous les rochers de la Seine infre. et du Calvados. D. - Z. M. Cherbourg. Le JoL. - Iles anglo-normandes. H. V. H. - Sur Patelles, Z. M. 
ile Callot. J. Ch. - Roscoff. Sir. - Le Conquet. Langeron. - Z. I. banc du Moulin, Melon, Corson. CRN. - Au pied de la falaise du Toulinguet (Crozon). Dét. Reinbold. J. CH. - Le Croisic. FL. Biarritz, au pied des falaises du Port-Vieux. S.

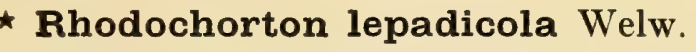

Indiqué à Swanage (Angleterre) par BatT.

Rh. membranaceum Magnus.

$=$ Rh. entozoicum Giardii Reinsch.

Sur Hydraires et Bryozoaires (Sertularia abietina), EP. à Wimereux, Croy. De Block. - Bassure de Baas, au large de Boulogne. Giard. - EP. à Berck-sur-Mer, Port-en-Bessin, Grandcamp. D. - Iles anglo-normandes. Jersey 1903. H. V. H. - Casiers à homards, Guéthary. J. CH.

Rh. mesocarpum (Carm.) Kjellm.

= Callithamnion (Carm.) Harv.

Sur coquilles, rade de Brest. CRN.

* Rh. minutum (Suhr.) Rke.

Weymouth. BATT.

* Rh. pallens (Zan.) Hauck.

= Callithamnion Zan. - Thamnidium Hauck.

Côtes du Devonshire. Bat'T.

Cette espèce a été aussi trouvée en Dalmatie.

Rh. Rothii (Turt.) Näg.

= Callithamnion Lyngb. - Thamnidium Thur. - Trentepohlia Harv.

Couvre la voûte d'une immense grotte de la falaise, près du phare du cap Gris-Nez. De Block. - Gris-Nez; Wimereux, la Rochette; la Crèche. D. - La Courtine (Etretat) au pied des falaises; Bruneval. Bernard. - Luc, Port-en.Bessin. Chauv. Côtes du Calvados. Len. - Z. S. musoir S. du port militaire; jetée E. du port du Commerce à Cherbourg; rochers de la caverne Ste-Colombe à Gréville. Le Jol. - Iles anglo-normandes. Jersey 1902. H. V. H. - Rochers et grottes marines; banc de St. Marc et Ste-Anne. CrN. - Rochers près de Vannes (Morbihan). Dumont. - 
Le Croisic. Fu. - Grottes de la grande côte (Croisic). D. - La Teste-de-Buch. Chantelat. - Port-Vieux et rochers du Casino à Biarritz; San Vicente (los Rosales), Rivadeo (las Garragas). S.

Rhodochorton sparsum (Carm.) Kjellm. Näg.

= Callithamnion Carm. Harv. (dans Crn.) - Acrochaetium

Kervallon. CrN.

Callithamnion acrospermum J. Ag.

$=$ C. roseum Chauv. - C. Dudresnayi Crn. - C. affine Harv. Ceramium Bonnem. - Dans l'exsiccata Desmaz. je trouve Call. roseum Len. donné comme une forme de Call. acrospermum : Port (Calvados). Desmaz. - Crouan réunit Call. acrospermum J. Ag. et Call. affine Harv. sous le nom de Call. Dudresnayi Bonnem. - D’après J.Ag., ce dernier représente une espèce formée par rassemblement et très douteuse.

Porte de la retenue à Fécamp. D. - Arromanches, Port-enBessin. Chauv. - Côtes du Calvados. Len. - Roscoff. Sir. Camfrout, passage de Plougastel, Lauberlac'h, l'Odet, à l'embouchure des rivières marines. CRN.

A. minus Bonnem. - St. Malo, St. Pol de Léon, Brest. Desmaz.

$$
\text { C. arbuscula (Dillw.) Lyngb. }
$$

= Phlebothamnion Kütz.

Iles anglo-normandes? (Liste de Melle Lelièvre). H. V. H.

\section{G. bipinnatum Crn.}

Dragué, rade de Brest. Crn.

D'après J. Ag. espèce à rechercher.

\section{G. Brodiæi Harv.}

= Call. guttatum J. Ag. - Ceramium Bonnem.

Barfleur. Herb. J. B. de Bruxelles. - Cherbourg. De la Pylaie. - Z. I. batterie du Diable, anse du Minou. CRN. - Banc du Château de Brest. Desmaz. - Penmarc'h. Bonnem. 


\section{Callithamnion corymbosum (Sm.) Lyngb.}

= Call. versicolor Ag. - Ceramium fruticulosum Bonnem. Ceramium Ag. - Ceramium byssoides Ducl. - Phlebothamnion corymbiferum Ktz.

Luc, Arromanches. Chauv. - Quihot, Grandcamp. D. - Côtes du Calvados. Len. - Z. M. et Z. I. plage Napoléon, bouées du port militaire de Cherbourg, St. Vaast. Le JoL. - Iles anglo-normandes. H. V. H. - St. Pol de Léon. CRN. - Roscoff. Sir. - Le Conquet. Langeron. - Bancs de St. Marc et du Moulin-Blanc. Crn. - Le Croisic. Fl. D. - La Teste-de-Buch. Chantelat. - Sur Helminthocladia, Guéthary. S. LE JoL.

A. amphicarpum Thur. - Plages Napoléon et des bains.

B. byssoides Crn. - Rade de Brest. CRN.

C. secundatum Harv. - Plages Napoléon et des bains, audessous des rochers de Longlet. Le JoL.

\section{C. decompositum (Grat.) J. Ag.}

$=$ Mertensia Grat.

Banc de St. Marc. CrN.

C. Dudresnayi Crn.

$=$ Call. affine Harv. dans Crv. Flor. Finist. p. 137.

Jersey 1904. De Bellaing dans H. V. H.

G. fallax Crn.

Dragué, rade de Brest. CrN.

D'après J. Ag. espèce à rechercher.

\section{G. fasciculatum Harv.}

= Pleonosporium Borrerii fasciculatum Holm. ot Batt. Call. Borrerii Ag.

Kervallon. CRN.

\section{G. Felixii Gaill.}

= Ceramium Gaill.

Dieppe. Gaillon. 
Gallithamnion fruticulosum J. Ag.

= Call. Hookerii fruticulosum Aresch. - Ceramium Ktz. Call. pyramidatum Liebm.

Roscoff. Melles V. et K. - Banc du Moulin-Blanc. CRN. Biarritz, Guéthary, St. Jean de Luz, Rivadeo. S. - Jersey 1904, sur Chondrus crispus. H. V. H.

\section{C. gallicum Näg.}

= Maschalosporium Näg. - D'après Nägeli, c'est l'espèce distribuée par Crouan, Algues du Finistère No 154, comme Call. Brodiaei. Il y aurait donc lieu de revoir dans les herbiers cette dernière espèce.

La Corogne, sur Corallina mediterranea et Plumaria elegans. S.

C. granulatum (Ducl.) Ag.

$=$ Call. spongiosum Harv.

EP. à Ostende. W. - Wimereux. Moniez. - Z. M. et Z. I. Cherbourg. Le JoL. - Mes anglo-normandes. H. V. H. - Roscoff. Sir. - Z. I. banc du Château, le Minou. CRN. - Z. S. rochers battus de la grande côte (Croisic). D. - Biarritz, Guéthary, St. Jean de Luz, San Vicente, Rivadeo. S. - Le Ferrol. CoL.

\section{G. Hapalidii Crn.}

Dragué, rades de Brest et de Camaret, sur Melobesia. CRN.

\section{Hookerii (Dillw.) Harv.}

= Call. lanosum Harv. - Phlebothamnion spinosum Ktz.

Z. I. rarement Z. M. Wimereux, Fécamp, Yport. D. - St. Jouin, le Havre. Bernard. - Englerqueville, Ste-Honorine. Bertot. Gatteville. Herb. Zan. - Z. M. et Z. I. Cherbourg. Le Jol. - Iles anglo-normandes. H. V. H. - Roscoff. Sir. - Bancs du Château et de St. Marc. Crn. - Le Croisic. Fl. - St. Jean de Luz, San Vicente. S.

\section{C. humile Ktz.}

Baie de Bertheaume. CRN.

Espèce du Cap de Bonne Espérance (Pappe). 
Callithamnion Lamourouxii (Duby.) J. Ag.

= Herpothamnion Näg. - Ceramium Duby.

Côtes du Calvados. Lamour.

\section{G. parvulum Crn.}

Dragué, rade de Brest. CrN.

\section{C. polyspermum Ag.}

= Call. scopulorum Lloyd. - Call. roseum Ag. - Call. Grevillei Harv.

Sur pilotis du port d'Ostende. Kx. - Bénouville. D. - Murs des quais du Havre. Dupray. - Z. M. Cherbourg. Le Jol. - Jersey 1903. Iles anglo-normandes. H. V. H. - Z. M. Laninon. CRN. - Le Croisic. FL.

A. minus Debray. - Z. S. Wimereux, la Rochette et Croy; port en eau profonde de Boulogne. D. - Antifer. Bernard.

\section{G. pseudo-byssoides Crn.}

Kervallon. CRN.

D’après J. Ag. espèce à rechercher. Espèce inconnue de De Toni.

$$
\text { C. roseum (Roth.) Harv. }
$$

= Phlebothamnion Kutz. - Call. octosporum Ag.

Arromanches. D. - Port-en-Bessin. Chauv. - Côtes du Calvados. BRÉB. - Z. M. plage Napoléon. LE Jol. - Iles anglo-normandes. H. V. H. - Z. I. Camfrout, Lauberlac'h, le Passage. Crn. - Le Croisic, port et chenal. FL. - Grande côte (Croisic). D. - La Barquera (San Vicente) sur Fucus vesiculosus. S.

$$
\text { G. rupicolum Dupray. }
$$

Le Havre. Dupray.

$$
\text { C. scopulorum Ag. }
$$

$=$ Ceramium Ag. - Call. roseum tenue Lyngb. - C. roseum scopulorum Lenorm.

Sur pilotis du port d'Ostende. Landszweert, dans Herb. J. B. de 
Bruxelles. - Z. S. Port-en-Bessin, Arromanches. Chauv. - Calvados. J. Ag. - Brest. Herb. Zan.

Les plantes publiées sous ce nom par Chauvin et Crouan sont de jeunes Call. polyspermum.

\section{Callithamnion serpens Crn.}

Dragué, rade de Brest. CRN.

D'après J. Ag. espèce à rechercher. D'après Batt. type du genre nouveau Hymenoclonium, adopté par De Toni.

\section{G. simplex Crn.}

Laninon, St. Mare, sur Zostera ot Solenia compressa. CrN.

Est-ce une espèce de Bangiacée? (De Toni).

\section{G. tetragonum Ag.}

= Call. Harveyanum J. Ag.

EP. à Ostende. W. - Wimereux. Moniez. - Fécamp, Yport. D. - Bénouville. Bernard. - Etretat. D. - Bruneval. Bernard. - Quihot. D. - Langrune. Chauv. - Arromanches, Grandeamp. D. - Z. I. Cherbourg. LE JoL. - Iles anglo-normandes. H. V. H. - Granville. Len. - Roscoff. Sir. - Le Mingant, le Minou. CRN. - Le Croisic. Fu. D. - Sur Gelidium corneum, Biarritz. Postels. - Guéthary, St. Jean de Luz. S. - La Corogne. LAz.

A. brachiatum J. Ag. (= Call. brachiatum Harv.) - Langrune. Chauv. - Digue de Cherbourg. Le Jol. - Mes anglo. normandes. H. V. H. - Le Délec, le Conquet, Laber-Ildut, Argenton. CRN.

Les deux formes tetragonum et brachiatum sont réunies par Bonnemaison et par Harvey sous le nom de Ceramium ou Call. brachiatum, et par De Toni sous le nom de Call. tetragonum Harv.

\section{G. tetricum (Dillw.) Ag.}

EP. à Ostende. Kx. - Petites-Dalles, Fécamp, Yport, Etretat. D. - Le Tilleul. Bernard. - Luc. D. - Z. M. et Z. I. Cherbourg. Le Jol. - Iles anglo-normandes. Batt. H. V. H. - Granville. Len. - Roscoff. Sir. - Z. I. rade de Brest. CRN. - Camaret. J. Сн. Belle-Isle. Jouan. - Morbihan. Lelièvre. - Le Croisic. Fu. D. Z. I. Biarritz, Guéthary, St. Jean de Luz, San Vicente, Gijon, Rivadeo, la Corogne. S. 
Callithamnion tripinnatum (Grat.) Ag.

= Phlebothamnion Ktz. - Mertensia Grat.

Côtes de France. Grateloup. - Sur Chondrus crispus, chenal de l'île Verte à Roscoff. Dét. Heydrich. J. CH. - Kervallon, entrée du port militaire de Brest, dragué dans le chenal de la rade de Brest. CRN. - Sur Araignée de mer à Guéthary. S.

\section{Phlebothamnion pachycaulon Ktz.}

Voir Callithamnion, De Toni, Floridées, p. 1340.

Golfe de Gascogne. De Martens dans Col.

\section{Seirospora byssoides Arnott.}

= Ceramium polyspermum Crn. - Callithamnion Arnott. Phlebothamnion Ktz.

EP. à Nieuport. Kx. - St. Valéry-en-Caux, Petites-Dalles, Fécamp, Yport, St. Jouin, Port-en-Bessin. D. - Arromanches. LEN. - Z. I. sur Codium tomentosum et Corallines, Hommet, Querqueville. Le JoL. - Iles anglo-normandes. H. V. H. - Le Conquet. Langeron. - Bane de St. Mare et rade de Brest, sur Gracilaria compressa. CRN. - Sur Araignée de mer à Guéthary. S.

A. plumosa (= Callithamnion plumosum Ktz.) - Arromanches. Herb. J. B. de Bruxelles. - Océan atlantique du Nord. Hauck.

$D^{\prime}$ après J. Ag. cette forme est à rechercher.

\section{S. flaccida Ktz.}

$=$ Callithamnion Rabenhorstii (Ktz.) Crn.

Dragué, rade de Brest. CRN.

D’après J. Ag. espèce à rechercher.

\section{S. Furcellariæ J. Ag.}

$=$ Callithamnion J. Ag. - Call. byssoides Aresch.

Banc du Moulin-Blanc. CrN.

\section{S. Gaillonii Crn.}

$=$ Ceramium Crn. - Phlebothamnion Ktz. - Callithamnion J. Ag. - Call. spinosum Crn.

Roscoff. Sir. - Z. I. entrée du port militaire de Brest. CRn. Commes, Port-en-Bessin. Bertot.

Forme avec renflements sphériques intercalaires sur les 
filaments; sur Cladophora rupestris, arrosé d'eau douce à marée basse, Port-en-Bessin. D.

\section{Seirospora Griffithsiana Harv.}

= Callith. seirospermum Griff. - Call. versicolor seirospermum Harv. - Call. versicolor (Draparn.) Ag.

Côtes de France. Nägeli. Lebel. - St. Vaast. Herb. J. B. de Bruxelles. - Sur Arthrocladia villosa, EP. à Cherbourg. Thur. Iles anglo-normandes. H. V.H. - St. Pol de Léon; bancs de St. Marc et du Moulin-Blanc. CrN.

\section{S. interrupta (Sm.) Schm.}

= Callithamnion Ag. - Call. byssoides seirosporifera Holm. Dragué, rade de Brest. CRN.

S. tenuissima (Bonnem.) Ktz.

= Callithamnion (Bonnem.) Ktz. - Ceramium Bonnem.

Iles anglo-normandes. H. V. H. - Z. I. banc du Château, SteAnne, sur Corallines. CrN. - Belle-Isle. Luoyd.

Compsothamnion gracillimum (Harv.) Näg.

= Callithamnion Harv. - Call. thuyoides Chauv. ?

Wimereux, Croy; Petites-Dalles, Yport, Fécamp. D. - St. Jouin. Bernard. - Luc. Chauv. - Arromanches. D. - Côtes du Calvados. LEN. - Z. I. banes de St. Marc et du Moulin-Blanc. CRN. - Z. I. Nacqueville. Born. - St. Vaast. Le Jol.

\section{G. thuyoides (Sm.) Näg.}

= Callithamnion gracillimum Ag. - Callithamnion Ag. Call. tripinnatum Harv. - Ceramium Bonnem.

Côtes du Calvados. Len. - Iles anglo-normandes. H. V. H. Roscoff. Melles V. et K. - Z. I. banc du Château, batterie du Fer-àcheval, sur Corallines. CRN. - Sur Araignée de mer à Guéthary. S. - San Vicente de la Barquera. Laz.

Plumaria elegans (Bonnem.) Schm.

= Ptilota Bonnem. - Pt. sericea Harv. - Autrefois regardé comme une var. de Pt. plumosa Ag. (d'après Batt.) - Pt. plumosa tenuissima Ag. - Pt. plumosa capillaris Grev. 
EP. à Nieuport. Kx. - Z. I. Wimereux, Croy. Giard. - PetitesDalles, Fécamp, Yport. D. - Bénouville. Bernard. - Etretat. D. - Antifer, Bruneval. Bernard. - Arromanches. D. - La Hougue. D. - Z. M. Cherbourg. Chauv. - Iles anglo-normandes. H. V. H. - Granville. Chauv. Len. - Z. I. Duon (Roscoff) J. Ch. - Roscoff. Sir. - Z. I. sous le Polygone, le Délec, Laber-Ildut, Melon. CRN. Pied des falaises de Toulinguet et de Pen-Tir (Crozon). J. Сн. Belle-Isle. Desmaz. - Rivadeo, la Corogne. S.

\section{Plumaria Schousboei (Born.) Schm.}

\section{= Ptilota Born. - Callithamnion elegans Schousb.}

Biarritz, Z. S. grottes au pied des falaises. Thur. Born. LespiNASSE. - San Vicente, falaise de los Rosales. S.

\section{Ptilota hypnoides Harv.}

Jersey. Herb. Bréb. dans Desmaz.

Espèce de Californie.

\section{Pt. plumosa (L.) Ag.}

Le Pt. plumosa des anciennes flores d'Angleterre doit être rapporté à Plumaria elegans Bonnem. (d'après Batt.) Le vrai Pt. plumosa appartient à l'Atlantique du nord. Cependant je note les indications suivantes (à vérifier) :

EP. à Heyst et Blankenberghe. W. - EP. à Nieuport. Herв. J. B. de Bruxelles. - EP. sur tige de Laminaria, baie de Bertheaume. CrN. - Asturies et Galicie, Candas, Aviles, Concha de Artedo, la Corogne, le Ferrol. CoL.

\section{Antithamnion cruciatum (Ag.) Näg.}

= Callithamnion Ag. - Call. fragilissimum Ardiss. - Call. decipiens J. Ag.

Wimereux, Croy ; la Crèche, la Roche-Bernard (Boulogne). D. Arromanches. Chauv. - Querqueville; bouées du port militaire. Le Jol. - Jersey. J. Piquet dans H. V. H. - EP. au Conquet. LANGERon. - Sur Corallina, banc du Château de Brest, Laninon, banc de St. Marc. CRN. - Sur Araignée de mer à Guéthary. S.

A. pumilum Harv. - Sur Corallina, banc du Château de Brest. CRn. 


\section{Antithamnion floccosum (Müll.) Kleen.}

$=$ Callithamnion J. Ag. - Call. plumula Lyngb. (pro parte). - Call. Pollexfenii Harv.

Iles anglo-normandes. H. V. H.

A. plumula (Ellis.) Thur.

= Callithamnion Lyngb. (pro parte). - Ceramium Ag. Pterothamnion Näg.

EP. à Nieuport. Kx. - Z. I. Fécamp, Quihot, Arromanches, Grandcamp. D. - Côtes du Calvados. Len. - Z. I. plage Napoléon, rochers de Longlet, bouées du port militaire. LE JoL. - Iles anglonormandes. H. V. H. - St. Pol de Léon. Drsmaz. - Roscoff. Sir. J. CH. - Z. I. entrée du port de Brest, bancs de St. Mare et du Moulin-Blanc. Crn. - La Govelle (Croisic). D. - Biarritz. Endress. - Sur Araignée de mer à Guéthary. S.

A. crispum J. Ag. $(=$ A. crispum Thur. - Call. refractum Ktz.) - Z. I. plages Napoléon, des bains et des Flamands. LE JoL. - Carteret. Herb. J. B. de Bruxelles. - Roscoff. Melles V. et K. - Banc de St. Marc, Laninon, Argenton. CrN. - Le Croisic. Fu. Iles anglo-normandes. H. V. H. - EP. à Guéthary; Rivadeo, la Corogne. S.

Grouania attenuata (Bonnem.) J. Ag. Hauck.

$=$ Mesogloia Ag. - Cr. bispora Crn. - Cr. attenuata bispora

Z. I. entrée du port militaire; Flamands. LE JoL. - Iles anglonormandes. H. V. H. - Roscoff. Melles V. et K. - Z. I. Laninon, Ste-Anne; rade de Brest, dragué. CRN.

Spyridia filamentosa (Wulf.) J. Ag.

$=$ Sp. villosissima, Sp. attenuata, Sp. confervoides Zan. Ceramium Ag.

Z. M. Quihot, Grandeamp. D. - Luc, St. Vaast. Chauv. - Z. I. plages Napoléon et des bains; entrée du port militaire. LE JoL. Portbail. Herb. J. B. de Bruxelles. - Iles anglo-normandes. H. V. H. - Granville. Chauv. - Roscoff. Sir. - Dragué au large de Duon. J. Ch. - Z. I. Laninon. CRN. - Arotcha (Guéthary). S. - Le Ferrol. Col.

A. friabilis J. Ag. - Banc de St. Marc. CRN. - D'après J. Ag. 
on pourrait rapporter à cette forme : Sp. villosiuscula, Sp. hirsuta et $S p$. apiculata de Kützing.

B. Griffithsiana J. Ag. - St. Marc, Laninon. CrN. - Jersey 1904. H. V. H. - D'après J. Ag. on pourrait rapporter à cette forme : Sp. crassa, Sp. setacea, Sp. divaricata, Sp. cuspidata de Kützing.

C. simplicipilum J. Ag. - Indiquée sans localité dans l'Epicrisis; on pourrait y rapporter : $S p$. filamentosa et $S p$. villosa de Kützing.

\section{Ceramium acanthonotum Carm.}

Fécamp. D. - Etretat, Antifer. Bernard. - Hommet, Querqueville. LE JoL. - Iles anglo-normandes. H. V. H. - Z. M. batterie du Diable, le Minou. CrN. - Plage Valentin, le Pouliguen. D. Le Croisic. Lloyd.

\section{C. arborescens J. Ag.}

Côtes françaises. Chauv. Le Jol. - Sur Chorda filum, EP. à Wimereux. De Block.

\section{C. australe Sond.}

Sur Araignée de mer à Guéthary. S.

Espèce de la Nouvelle-Hollande et peut-être de l'Océan Pacifique tropical.

\section{G. ciliatum (Ellis.) Ducluz.}

Boulogne. Desmaz. - Fécamp, Etretat, Yport. D. - Luc, Arromanches. Chauv. - Cricqueville, Grandcamp. Bertot. - Côtes du Calvados. Len. - Z. M. Cherbourg. Le Jol. - Iles anglonormandes. H. V. H. - Roscoff. Sir. - Baie de Laninon, banc de St. Marc. CRN. - Camaret, au pied de la falaise E. du port. Dét. Reinbold. J. Сн. - Morbihan. Lloyd. - Le Croisic. FL. - Arotcha, près de Guéthary. $\mathrm{S}$.

Forme de 2 à $3 \mathrm{ctm}$. - Boulogne. Desmaz.

\section{C. circinatum J. Ag.}

$=$ C. decurrens Harv. - C. Biasolettianum Ardiss.

Grandcamp. D. - Roscoff, Roche du Loup, Z. I. sur Codium tomentosum. Dét. Heydrich. J. CH. - Baie du Moulin-Blanc, EP. CRN. 


\section{Ceramium Deslongchampsii Chauv.}

\section{= Gongroceras Ktz. - C. Agardhianum Griff.}

Ostende, sur les pilotis. W. - EP. à Nieuport. Kx. - Calais. BRÉB. - Z. S. et Z. M. Audresselles; Wimereux, la Rochette; Boulogne, le Tréport, Mesnilval, Petites-Dalles, Fécamp, Etretat, Antifer, Trouville, Luc, Arromanches. D. - Port-en-Bessin. Chauv. - Côtes du Calvados. LeN. - Z. M. rochers de Longlet, jetée de l'ouest. LE JoL. - Iles anglo-normandes. H. V. H. - Z. I. MoulinBlanc, passage de Plougastel. CRN. - Le Croisic. FL. D.

\section{G. diaphanum (Lightf.) Roth.}

$=$ C. elegans Grat. - Hormoceras cateniforme Ktz.

Sur Fucus vesiculosus à Blankenberghe (EP.?) EP. à Nieuport. Kx. W. - Z. I. Audresselles; Wimereux, Pointe-aux-Oies et Croy ; St. Valéry-en-Caux, Grandes-Dalles, Fécamp, Yport. D. - Antifer, St. Jouin. Bernard. - Luc, Arromanches, Port-en-Bessin. D. Portbail. Herb. J. B. de Bruxelles. - Cherbourg. Pelvet. Simon. - Iles anglo-normandes. H. V. H. - Roscoff. Sir. Melles V. et K. - Baie de Bertheaume, St. Mathieu, pointe de Corson, Argenton. Crn. - La Rochelle. Herb. Zan. - La Teste-de-Buch. Chantelat. - Guéthary. J. CH. - Rivadeo. S. - Le Ferrol. Col.

A. minus Crn. - Banc de St. Marc. Desmaz.

B. pilosum. - Côtes de Normandie, sans autre indication de localité. Chauv.

Chantelat indique à la Teste-de-Buch les variétés ciliatum et glabellum (??)

\section{G. divaricatum Crn.}

Tout à fait semblable, d'après J. Ag. à C. arachnoideum patentissimum HaRv.

Rade de Brest, sur feuilles de Zostère, EP. CRN.

\section{G. echionotum J. Ag.}

Z. M. et Z. I. Petites-Dalles, Yport, Etretat. D. - Le Tilleul, St. Jouin. Bernard. - Luc, Langrune, Arromanches, Port-en-Bessin, Grandcamp. D. - Z. I. Cherbourg. Le JoL. - Iles anglo-normandes. H. V. H. - Roscoff. Sir. - Le Conquet. Langeron. - Z. I. environs de Brest. CRN. - Morbihan. Lloyd. - Le Croisic. Fl. - Le Pouliguen. D. - San Vicente, la Corogne. Laz. 


\section{Ceramium fastigiatum Harv.}

$=$ Gongroceras Ktz. - C. coccineum Zan.

Iles anglo-normandes. H. V. H. - Banc du Moulin-Blanc. CRN. - EP. grande côte (Croisic). D.

\section{C. flabelligerum J. Ag.}

Sur pilotis et pierres, ports d'Ostende et de Nieuport. W. Gris-Nez, Wimereux, la Crèche, le Tréport, Criel, St. Valéry-enCaux, Petites-Dalles, Fécamp, Etretat. D. - Antifer. Bernard. Le Havre. Dupray. - Trouville, Port-en-Bessin. Bertot. - Z. M. murs du quai Napoléon à Cherbourg. LE JoL. - Iles anglonormandes. H. V. H. - Z. M. le Délec, Bertheaume. CRN. - Le Croisic. FL.

\section{C. gracillimum Griff. et Harv.}

$=$ C. diaphanum nanum, C. diaphanum tenue Ardiss. $-C$. flaccidum Harv.

Z. I. Arromanches. Chauv. - Port-en-Bessin, Grandcamp. D. St. Vaast. Le Jol. - Jersey 1903. H. V. H. - Laninon, Ste-Anne. Crn. - Le Croisic. Fl. D. - Bords du bassin, la Teste-de-Buch. Chantelat. - Biarritz, Guéthary, St. Jean de Luz, San Vicente, Gijon, Rivadeo, la Corogne. S. - Sur Cystoseira, Guéthary. J. Cн.

\section{? G. luxurians J. Ag.}

Ostende. Marissal.

Je trouve C. Iuxurians Desmaz: Brest. Desmaz.

\section{C. pedicellatum D. C.}

=C. rubrum pedicellatum Duby. - Callithamnion Ag.

Wimereux. J. Сн. - Luc. Chauv. - Cherbourg. Le Jol. Iles anglo-normandes. H. V. H. - Argenton. CrN.

A. minus Desmaz. - Calvados et Finistère, Z. I. Desmaz.

\section{C. pennatum Crn.}

Rade de Brest, EP. sur Halidrys siliquosa. CRN. D'après De Toni, espèce douteuse. 


\section{Ceramium rubrum (Huds.) Ag.}

EP. littoral belge. Kx. - Entre Ostende et Nieuport. W. Blanc-Nez, du Gris-Nez au Portel, Ault, Mers et tout le littoral de la Seine infre. et du Calvados. D. - Iles anglo-normandes. H. V. H. Portbail. Herb. J. B. de Bruxelles. - Roscoff. Sir. - Côtes du Morbihan. Crn. - Le Croisic. Fu. D. - La Rochelle. Herb. Zan. - La Teste-de-Buch. Chantelat. - Biarritz, Guéthary, St. Jean de Luz, de petite taille. S. - Fontarabie. Cou. - Rivadeo, la Corogne. S. - San Vicente de la Barquera. Laz. - Le Ferrol. Cor.

A. corymbiferum J. Ag. - Cherbourg. Le JoL. - Jersey 1903. H. V. H. - Banc du Moulin-Blanc. CrN.

B. decurrens J. Ag (=C. decurrens Harv.) - EP. à Wimereux, Fécamp. D. - St. Jouin. Bernard. - Luc. Chauv. - Grandcamp. D. - Arromanches. Herb. J. B. de Bruxelles. - Z. M. Cherbourg. Le Jol. - Z. I. St. Marc, Ste-Anne. CRn. - Anse du Corson. Desmaz. - Le Croisic. Fl. D.

C. diaphanum Desmaz. - EP. à Ostende. Kx. - Brest, banc de St. Marc. CRN.

D. fasciculatum J. Ag. - Argenton, Melon. CRN.

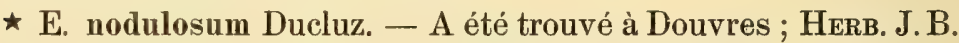
de Bruxelles. Ces nodosités ressemblent beaucoup à un parasite? Donné par De Toni comme syn. de C. rubrum.

F. proliferum J. Ag. (= C. botryocarpum Griff. Harv.) - Luc, Arromanches. Herb. J. B. de Bruxelles. - Cherbourg. Le Jol. Iles anglo-normandes. H. V. H. - St. Malo. Herb. J. B. de Bruxelles. - Baie de Bertheaume. Crn.

Forme de $2 \mathrm{ctm}$. - Cherbourg. Herb. J. B. de Bruxelles.

\section{C. strictum Grev. et Harv.}

$=$ C. diaphanum gracile Ardiss. - C. pellucidum, C. diaphanum minus Crn.

St. Vaast. Herb. Zan. - Z. I. plage Napoléon, rochers de Longlet. LE JoL. - Iles anglo-normandes. H. V. H. - Rade de Brest, dragué. CRN. - Le Croisic. FL. - Le Pouliguen. D. - Rivadeo. S.

A. delicatum J. Ag. (= Conferva Clem.) - Rade de Brest. Crn.

B. zostericola Thur. - Plages Napoléon, des Bains, des Flamands. Le Jol. - Le Conquet. Langeron. - Le Croisic. Fl. 


\section{Ceramium tenue (Ag.) J. Ag.}

$=$ C. rubrum tenue Ag.

Cherbourg. Le Jol. - Le Minou, Trégana. CRN.

C. tenuissimum (Lyngb.) J. Ag.

= C. nodosum Harv. - C. diaphanum tenuissimum Lyngb. - C. diaphanum rigidum Griff. et Harv. - C. arachnoideum patentissimum Crn.

Z. M. et Z. I. St. Jouin. Bernard. - Luc, Langrune, Arromanches, Grandcamp. D. - Z. I. plages des bains et des Flamands. LE Jou. - Iles anglo-normandes H. V. H. - Portbail. Herb. J. B. de Bruxelles. - St. Malo, St. Pol de Léon. Desmaz. - Roscoff. Sir. - Le Conquet. Langeron. - Z. I. St. Marc, Laninon; rade de Brest. CrN. - Chenal du Croisic. FL. D. - Gijon. S.

A. arachnoideum Ag. (=C. arachnoideum J. Ag. - C. tenuissimum Aresch.) - Iles anglo-normandes H. V. H.

B. uniforme Bonnem. - Banc du Château de Brest. Desmaz.

G. vimineum J. Ag.

=C. rubrum virgatum Ag. - Hormoceras siliquosum $\mathrm{Ktz}$. CRN.

Cherbourg. Le JoL. - Jersey 1903. H. V. H. - Z. I. Ste-Anne.

Microcladia glandulosa (Sol.) Grev.

= Delesseria Ag. - Microcladia tenuis Ktz.

Cherbourg, EP. Chauv. - EP. baie de Ste-Anne. Le Jol. Iles anglo-normandes. H. V. H. - Z. I. pointe N. de l'île Callot. J. Ch. - Roscoff. Sir. - Le Conquet. Langeron. - Environs de Brest, EP. Crn. - Quimper? Desmaz. - Le Croisic. Fl. - EP. Guéthary ; Z.I. Gijon, Rivadeo; la Corogne. S. - Le Ferrol. Cos.

\section{GLOIOSIPHONIÉES}

Gloiosiphonia.

Thuretella.

Schimmelmannia.

Gloiosiphonia capillaris (Huds.) Carm.

= Gigartina lubrica Lyngb.

Z. I. Cherbourg. Le JoL. - Iles anglo-normandes. H. V. H. Carteret. Herb. Zan, - Granville. Herb. Len. - St. Malo. Thur. 
- Roscoff. Melles V. et K. - Le Conquet. Langeron. - Z. I. Laninon, Ste-Anne, Bertheaume, St. Pol. CRN. - EP. grande côte (Croisic). D. - Belle-Isle. Herb. Len. - Sur Araignée de mer à Guéthary (limite sud). S.

\section{Thuretella Schousboei Schm.}

\section{= Crouania Thur.}

Guéthary, EP. en 1868. Thur. et Born. - Même localité, sur Araignée de mer. S.

\section{Schimmelmannia Schousboei J. Ag.}

$=$ Sch. ornata Schousb.

Sur Araignée de mer à Guéthary. S. - Epave à Guéthary, septembre 1904. J. Сн.

\section{GRATELOUPIACÉES}

Halymenia.

Grateloupia.
Cryptonemia.

Dermocorynus.

Halymenia ligulata (Wood.) J. Ag.

= Halarachnion Ktz.

Petites-Dalles, Fécamp, Yport; EP. à Courseulles et à Arromanches. D. - Réville, St. Vaast. LE JoL. - Iles anglo-normandes. H. V. H. - Carteret. Herb. Zan. - St. Malo. Thur. - Z. I. St. Marc, Laninon, St. Pol. CRN. - EP. à Guéthary. S. J. CH. - San Vicente, Candas, la Corogne. Laz.

A. acicularis Hauck. - Iles anglo-normandes. H. V. H. Portbail, St. Malo. Herb. Len. - Roscoff, dragué au large de Duon. J. Сн.

B. dichotoma Harv. - Dragué, chenal de la rade de Brest. CRN.

C. latifolia (=H. latifolia $\mathrm{Crn}$. - Est-ce une espèce ? demande J. Ag. - On remarque tous les passages des formes larges aux formes étroites, dit Thur.) - Roscoff. Melles V. et K. - Exemplaires hauts de 40 cent. et très larges, Brest, St. Vaast-la-Hougue; hauts de 60 cent. sur 10 de largeur, Barfleur, le Morbihan. 
Herb. Len. - Baie du Moulin-Blanc, EP. - Dragué, rade de Brest. CRN.

\section{Grateloupia dichotoma J. Ag.}

Laninon, sous le Polygone, Bertheaume, Argenton. CrN. Belle-Isle. Desmaz. - Guéthary. Lespinasse. - San Vicente, Gijon, Rivadeo, la Corogne. S.

\section{Gr. filicina (Wulf.) J. Ag.}

Petites-Dalles, Fécamp, Yport. D. - Bruneval. Bernard. EP. plage Napoléon. Le JoL. - Mes anglo-normandes. H. V. H. St. Malo. Herb. J. B. de Bruxelles. - Ste-Anne, le Minou, Bertheaume, Kervallon. CrN. - Le Croisic. Fu. - Plage Valentin, Pierre-plate, la Govelle (Croisic). D. - Biarritz, Guéthary, St. Jean de Luz, San Vicente, Gijon, la Corogne. S. - Portugalete. CoL.

A. intermedia Holm. et Batt. - Iles anglo-normandes. H. V. H.

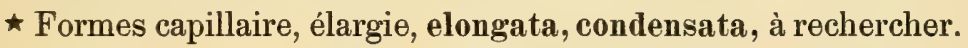

\section{Gr. minima Crn.}

Z. I. St. Marc, Laninon. CRn.

Gryptonemia Lomation (Bertol.) J. Ag.

$=$ Cr. Lactuca J. Ag.

Z. I. Rivadeo, Villasellan. S.

\section{C. seminervis J. Ag.}

$=$ Crypt. Lactuca seminervis Sauv. - État jeune du Cr. Lomation, d'après Bornet.

Pointe de Corson. CrN. - Biarritz. Born. - EP. à Guéthary ; San Vicente, au pied de la colline de Santa Catalina; la Corogne. S.

\section{Dermocorynus Montagnei Crn.}

Banc de St. Marc ; dragué, rades de Brest et de Camaret. CrN. 


\section{DUMONTIACÉES}

\section{Dumontia. Dudresnaya. Sarcophyllis.}

\section{Dumontia filiformis (Lyngb.) J. Ag.}

EP. entre Nieuport et la Panne. Kx. - Dieppe. Desmaz. - De Fécamp à St. Jouin. Bernard. - Arromanches. D. - Port-enBessin. Chauv. - Côtes du Calvados. Len. - Z. M. Cherbourg. Le JoL. - Iles anglo-normandes. H. V. H. - Roscoff, roche du Loup. Melles V. et K. - EP. à Perkiridic. J. CH. - Z. M. environs de Brest. CRN. - Le Ferrol. Col.

A. crispata J. Ag. (= Halymenia purpurascens crispata Grev. - Dum. filif. intestiniformis Lieb.) - Arromanches. HERB. J. B. de Bruxelles. - Z. M. Cherbourg. LE JoL. - Jersey 1903. H. V. H. Banc de St. Marc. CRN.

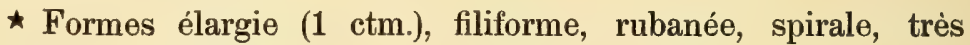
ramifiée. A rechercher.

\section{Dudresnaya coccinea Crn.}

= Nemalion Ktz. - Mesogloia Ag.

Z. I. Fécamp. D. - St. Vaast. Le Jol. - Cherbourg. Chauv. Iles anglo-normandes. H. V. H. - St. Malo. Thur. - Z. I. rade de Brest, dragué ; Laninon, banc de St. Marc. CRN. - Roscoff, dragué au large de Duon. J. CH. - Morbihan. Herb. ZAN. - Guéthary, EP. J. Ch. - Le Ferrol. Col.

Sarcophyllis edulis (Stackh.) J. Ag.

= Iridaea Bory. - Dilsea Stackh. - Schizymenia J. Ag. Sarcophyllis lobata Ktz.

Wimereux, Pointe-aux-Oies. D. - Dieppe. Desmaz. - PetitesDalles, Fécamp, Yport, Etretat. D. - Antifer, Bruneval. Bernard. - Lion. Chauvin. - Quihot, Langrune, Arromanches, Grandcamp. D. - Côtes du Calvados. Len. - Z. I. Port-en-Bessin, Cherbourg. Chauv. - Iles anglo-normandes. H. V. H. - Roscoff. Sir. - Z. I. ne découvrant pas, le Cerf; EP. à Perkiridic. J. CH. - Z. I. banc du Château, Ste-Anne. CRN. - Pen-Tir (Crozon). J. Сн. - Lorient. Herb. Zan. - Le Croisic. Fl. - Grande côte (Croisic). D. - Gijon, Rivadeo. S. - San Vicente. LAz. - Santander, Cudillero, Concha de Artedo, le Ferrol, la Corogne. Col. 


\section{NÉMASTOMAGÉES}

Calosiphonia.

Schizymenia.
Nemastoma.

Fastigiaria.

\section{Calosiphonia vermicularis Schm.}

\section{= Nemastoma J. Ag. - C. Finisterrae Crn.}

Iles anglo-normandes. H. V. H. - St. Malo. Thur. - Rade de Brest, dragué. CRN. - Sur Araignée de mer à Guéthary. S.

\section{Schizymenia Dubyi (Chauv.) J. Ag.}

= Halymenia Chauv. - Callymenia Harv. - Euhymenia Ktz.

Petites-Dalles, Yport. D. - St. Jouin. Bersard. - Fécamp, Cherbourg. Chauv. - Z. I. entrée du port militaire, Hommet, Digue. LE JoL. - Iles anglo-normandes. H. V. H. - Roscoff. SiR. - Z. I. banc du Château, batterie du Fer-à-cheval. CRN. - Z. I. le Croisic. FL. - Grande côte, Pierre-plate (Croisic). D. - Biarritz, Guéthary, St. Jean de Luz. S. - St. Jean de Luz, jetée Ste-Barbe; atteint parfois 40 et 50 cent. A la fin de mai, déjà décoloré et décomposé. J. Сн. - San Vicente, Gijon, Rivadeo. S. - La Corogne. Laz.

\section{S. minor J. Ag.}

Z. I. fort Mingant. CRN. (Born).

Il n'y a pas de caractères distinctifs réels entre Sch. Dubyi, minor et cordata

Nemastoma marginifera J. Ag.

= Platoma Schm.

Pointe de Corson, anse du Minou. CRN. - Biarritz. Thur. Guéthary, EP. abondante en septembre; en place, la Goureppe. J. Сн. - Z. I. San Vicente, Gijon. S.

\section{Fastigiaria furcellata Stackh.}

\section{= Furcellaria fastigiata Huds.}

EP. entre Ostende et Nieuport. Kx. - Gris-Nez. D. - Dieppe. Duboc. - Petites-Dalles, Senneville, Fécamp, Yport. D. - Etretat, Bruneval. Bernard. - Luc, Langrune, Arromanches, Grandcamp. D. - Z. I. Cherbourg. LE JoL. - Iles anglo-normandes. H. V. H. Roscoff. Sir. - Le Conquet. Langeron. - Z. I. environs de Brest. 
CrN. - Le Croisic. FL. - EP. la grande côte (Croisic). D. - Gijon. S. - San Vicente. Laz. - Santander, Conchas de Artedo. Col.

Forme grêle, à paquets de rameaux fastigiés au bout des branches. - Côtes de la Manche. Herb. Malebranche.

\section{RHIZOPHYLLIDÉES}

Polyides.

Contarinia.

Polyides lumbricalis J. Ag.

= Furcellaria Ktz. - Polyides rotundus Grev.

EP. à Blankenberghe et à Nieuport. Kx. - Gris-Nez, Audresselles, Wimereux, le Portel, le Tréport et tout le littoral de la Seine infre. D. - Luc, Arromanches, Cherbourg. Chauv. - Iles anglonormandes. H. V. H. - Roscoff. Sir. - Z. I. le Minou. CRN. Lorient. Herb. ZaN. - Le Croisic. FL. D. - Santander, San Vicente, Comillas, Candas, la Corogne. Laz. - Concha de Artedo. Cox.

\section{Contarinia pulcherrima Crn.}

$=$ Rhododiscus Crn. - D'après J. Ag. très voisin de C. peyssonneliiformis (Zan.) J. Ag.

Rades de Brest et de Camaret, dragué. CRn.

\section{SQUAMARIÉES}

Petrocelis.

Cruoria.

Cruoriopsis.

Cruoriella.
Peyssonnelia.

Hæmatocelis.

Rhododermis.

\section{Petrocelis cruenta J. Ag.}

= Cruoria pellita Harv. non Lyngb.

Sur silex. Fécamp. D. - Etretat, Antifer, Bruneval. Bernard. - Arromanches. Herb. Zan. - Z. M. Querqueville, Flamands, île Pelée. Le JoL. - Iles anglo-normandes. H. V. H. - Roscoff. Sur 
roches ne découvrant jamais : Kaïnou, le Loup etc. J. Сн. - Z. M. banc du Château, Ste-Anne. CRN. - Belle-Isle. Lloyd, Algues de l'ouest, No 233. — Gijon, cap Torres ; Rivadeo. S.

Gruoria adhærens Crn. dans J. Ag.

= Nemalion Crn. - Cruoria pellita Desmaz. exsicc. 1613, non Fries, d'après Crn. - Cr. pellita (Lyngb.) Fries, d'après Hauck et Le Jolis.

Bancs du Château et de St. Marc à Brest. CRn. Algues du Finist. No 234.

Cr. pellita (Lyngb.) Fries.

Entrée du Port militaire, Hommet, Querqueville. Le JoL. - Le Croisic. FL.

Cr. purpurea Crn.

$=$ Contarinia cruoriiformis Crn. - Cr. rosea Crn. var: purpurea Batt.

Rade de Brest, dragué ; sur Lithothamnion. CRN.

Gr. rosea Crn.

= Contarinia Crn. - Cr. stilla Kuck.

Rade de Brest, dragué. CRN.

Gruoriella armorica Crn.

Rade de Brest, dragué, sur Melobesia et Haliotis. CRN.

* Cruoriopsis gracilis Batt.

= Plagiospora Kuck. - C. cruciata Zan?

Dragué à Plymouth. BAtr.

$\star$ Gr. Hauckii Batt.

Plymouth, dragué. BatT.

Peyssonnelia atropurpurea Crn.

$=$ Haematocelis Schousboei J. Ag. (pro parte).

Fécamp, Yport, Bruneval, Quihot, Grandcamp. D. - Z. I. Cherbourg. LE JoL. - Iles anglo-normandes. H. V.H. - Toulbroc'h, Trégana. CrN. - Biarritz, Guéthary, St. Jean de Luz. S. 


\section{Peyssonnelia Dubyi Crn.}

Fécamp, Bénouville, Luc, Port-en-Bessin, Grandcamp. D. Iles anglo-normandes. H. V. H. - Z. I. banc de St. Marc, Laninon. CRN. - Biarritz. S.

\section{P. Harveyana Crn.}

Rade de Brest, EP. Crn. - Sur Patelles, Biarritz, Guéthary, St. Jean de Luz. S.

* P. Rosenvingii Schm.

Côtes S. de l'Angleterre. Batr.

* P. rubra (Grev.) J. Ag.

= P. Dubyi Harv. pro parte. - Zonaria Grev.

Plymouth, dragué. BATT.

P. rupestris Crn.

Fort du Mingant. CrN.

\section{P. squamaria Dene.}

Côtes du Calvados. BrÉB. Herb. J. B. de Bruxelles. - Biarritz, Guéthary, St. Jean de Luz; les fragments sont portés par les Oursins. San Vicente, Gijon. S. - Portugalete, Santander. CoL.

\section{Hæmatocelis fissurata Crn.}

Rade de Brest, sur Pecten, dragué. CRN.

\section{H. parasitica Crn.}

Baie de Bertheaume, Laber-Ildut, sur Cystoseira. CRN.

H. rubens J. Ag.

= Haematophlaea Crouanii Crn.

Laber-Ildut. CRN.

\section{Rhododermis elegans Crn.}

Sur Zostère, Tatihou. Malard et Kuckuck. - Rade de Brest, dragué. CRN.

A. forma Georgii (Batt.) Heydr. (= Rhodophysema Georgii Batt. - Rhododermis Van Heurckii Heydr.) - Sur Zostère, baie St. Aubin à Jersey 1903. H. V. H. 


\section{^ Rhododermis parasitica Batt.}

Plymouth. BatT.

\section{CORALLINÉES}

Schmitziella.

Choreonema.

Melobesia.

Dermatolithon.

Lithophyllum.

Lithothamnion.

Epilithon.
Corallina.

Amphirhoa.

Eleutherospora.

Hapalidium.

Lithocystis.

Hildenbrandia.

Schmitziella endophlæa Born. et Batt.

Dans Cladophora pellucida. Fécamp, Bruneval, Grandcamp. D. - Granville, St. Malo, Morbihan. Herb. Len. - Jersey 1902. H. V. H.

- Duon (Roscoff.) J. Ch. - Biarritz, Guéthary, St. Jean de Luz. S.

\section{Ghoreonema Thuretii Schm.}

\section{= Melobesia Born.}

Sur Corallina squamata, Jersey 1903. H. V. H. - Le Croisic. FL. - Guéthary. J. Сн. - Sur Corall. corniculata : Rivadeo, la Corogne. S.

\section{Melobesia callithamnioides Falk.}

Côtes S. de l'Angleterre. Batт.

\section{Gorallinæ Crn.}

Jersey 1902. Iles anglo-normandes. H. V. H. - Banc du Château, Laninon. CRs. - Sur Corallina officinalis, le Croisic. FL. - Sur Corall. mediterranea, Rivadeo, la Corogne. S.

\section{M. farinosa Lamour.}

Iles anglo-normandes. H. V. H. - Sur Cystoseira et Zostère, Grandcamp. D. - Sur Zostère, Cherbourg. Le Jol. - Roscoff. Sir. 
- Duon, sur Cladophora rupestris. J. Сн. - Environs de Brest. CRn. - Le Croisic. Fl. - Biarritz, Guéthary, St. Jean de Luz, San Vicente, Gijon, Rivadeo. S.

\section{Melobesia inæquilaterale Solms.}

Sur Aglaosphenia, établi lui-même sur Halidrys. Tatihou. MaLARD et KucKUCK.

\section{Le Jolisii Rosan.}

Sur Zostère, Bruneval. D. - Cherbourg. Herb. ZAN. - Jersey 1903. Iles anglo-normandes. H. V. H.

M. membranacea (Esper.) Lamour.

= Corallina Esper. - Lithothamnion Fosl. - Epilithon Heydr. - M. corticiformis Ktz. - Hapalidium Hildenbrandioides Crn.

Sur Zostera à Ostende. W. [Les Zostera arrivent comme EP. sur le littoral belge. CRÉPIN.] - EP. sur d'autres Algues. De W. Gris-Nez, Wimereux. D. - Yport, Bénouville, Etretat, Bruneval. Bernard. - Cherbourg, sur Zostera et Laminaria. Le Jol. Jersey, sur Algues diverses, 1903. Iles anglo-normandes. H. V. H. Environs de Brest. CrN. - Sur Laurencia pinnatifida, le Croisic. FL. D. - Biarritz, Guéthary, St. Jean de Luz, San Vicente, Gijon, Rivadeo, la Corogne. S.

M. pustulata Lamour.

$=$ Dermatolithon Foslie.

Etretat. Bernard. - Arromanches. Herb. J. B. de Bruxelles. - Cherbourg. LE JoL. - Jersey 1903. Iles anglo-normandes. H. V. H. - Ste-Anne. Cro. - Biarritz, Guéthary, St. Jean de Luz, San Vicente, Gijon, Rivadeo, la Corogne. S.

M. verrucata Lamour.

Environs de Brest. CRn.

Douteuse espèce. Peut-être M. farinosa.

M. zonalis (Crn.) Fosl.

$=M$. myriocarpa Crn. - Donné dans Crouan, d'après M. Heydrich, sous les noms de Hapalidium coccineum (pro parte), 
$H$. confervoides, $H$. roseum et $H$. zonale. - Foslie, dans Rem. sur les Melobesia de l'Herb. Crouan, dit que le M. zonalis ne diffère guère de $M$. membranacea.

Dragué rade de Brest. CRN.

\section{Dermatolithon hapalidioides (Crn.) Fosl.}

= Melobesia Crn. - Melobesia simulans Crn. - Il serait intéressant de voir sur exemplaires authentiques si c'est vraiment une espèce, ou bien l'état jeune de Lithothamnion Lenormandii, m’écrit M. Heydrich.

Banc de St. Mare; dragué rade de Brest. CRN.

A. confine (Crn.) Fosl. (= Melobesia confinis Crn.) - Cherbourg. Le JoL. - Z. S. forts du Diable et du Mingant. CRN.

\section{D. macrocarpum Rosan.}

A. Laminaria (Crn.) Fosl. (= Melobesia Laminariae Crn.) Cherbourg. LE JoL. (no 255 des Algues de Cherbourg). - Fort Mingant, Porspoder, Argenton. CRN. - Sur Laminaria Cloustonii, San Vicente, la Corogne. S.

Est-ce bien une espèce? Peut-être une forme jeune d'un Lithothamnion installé sur Laminaire. (Heydrich).

\section{Lithophyllum expansum Phil.}

\section{= Melobesia stictiformis Aresch. - L. giganteum Zan.}

Iles anglo-normandes (Guernesey d'après Ed. Marquand). H. V.H.

Ce n'est pas une espèce du Nord de la France, m'écrit Heydrich, mais seulement de la Méditerranée.

\section{L. incrustans (Phil.) Heydr.}

= Lithothamnion polymorphum Crn. - Lithoth. depressum Crn. (Florule p. 151). - Lithoth. crassum Unger.

Jersey 1902. Iles anglo-normandes. H. V.H. - Toulbroc'h. CRN. - Toutes les côtes basques. Lespinasse. - Z. S. encadre les Oursins, Biarritz, Guéthary, St Jean de Luz, San Vicente, Rivadeo, la Corogne. S.

Cette espèce semble avoir été confondue souvent avec Eleutherospora polymorpha (L.) Heydr., notamment par Johnston, Harvey, Le Jolis, Rosanoff, Hauck, Areschoug..... Ainsi Hauck p. 272 réunit les deux sous le nom de Lithoth. polymorphum (L.) Aresch. en une seule liste de synonymes. Le Jolis et Debray ne citent que Lithoth.polymorphum, Sauvageau seulement Lithoth. incrustans. Mais Batters, d'accord avec Foslie et Heydrich, donne Lithoph. incrustans Fosl. et Phymatolithon polymorphum Fosl, en deux genres différents. Crouan a aussi distingué les deux espèces. 
A. fasciculatum (Lamark) Heydr. (= Lithothamnion fasciculatum (Lamark) Aresch. - Melobesia fasciculata Harv.) - Côtes atlantiques du N. de l'Europe. - Rade de Brest, dragué. CRN. San Vicente, la Corogne. LAz.

B. flabellatum Heydr. - Z. S. Guéthary. J. Сн.

C. Harweyi Fosl. - Z. S. Guéthary. J. Сн.

D. sub-dichotomum Heydr. - Z. S. Guéthary. J. CH.

Forme de chou-fleur, intermédiaire entre Harweyi et dichotomum. - Z. S. Guéthary. J. Сн.

\section{Lithophyllum racemus (Lamark.) Fosl.}

= Lithothamnion Aresch. - Millepora Lamark. - Lithothamnion crassum Phil.

Rade de Brest, près du Bendic, dragué. CRN.

\section{L. tortuosum (Esp.) Fosl.}

$=L$. cristatum Menegh.

Biarritz, Guéthary, St. Jean de Luz, San Vicente, Gijon, Rivadeo, la Corogne. S.

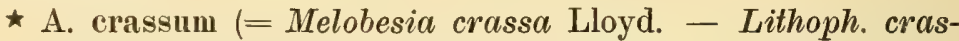
sum Rosan. - Lithoph. tortuosum [Esp.] Fosl. forma crassum [Lloyd] Hauck.) - Probablement avec le type; à rechercher.

Lithothamnion calcareum (Ell. et Sol.) Aresch.

= Lithophyllum (Pall.) Ell. et Solier. - Melobesia Harv. Lithophyllum coralloides Crn.

Dragué sur les Platiers, au large d'Etaples, par 30 à $40 \mathrm{~m}$. D. Roscoff, dragué entre les Bisayers et Duon. J. Сн. - Rade de Brest; embouchure de la rivière de Morlaix, dragué pour l'agriculture. CRN. - Dragué en abondance au large de Concarneau. J. CH. - Dragué entre le Pouliguen et le Croisic. D.

Les formes suivantes ont été reconnues par Heydrich dans le total d'un dragage fait à Duon :
A. compressum.
B. crassum.
C. mamillosum.
D. minutum.
E. subsimplex.
F. subvalidum. 


\section{Lithothamnion Lenormandii (Aresch.) Fosl.}

$=$ Lithophyllum (Aresch.) Rosan. - Melobesia Aresch.

Gris-Nez, Audresselles, Fécamp. D. - Bruneval, St. Jouin. Bernard. - Port-en-Bessin, Grandcamp. D. - Arromanches. Herb. J. B. de Bruxelles. - Cherbourg. Le JoL. - Jersey 1903. Iles anglonormandes. H. V. H. - Z. M. baie Ste-Anne près de Brest. Crn. - Biarritz, Guéthary, St. Jean de Luz. S. - San Vicente de la Barquera, la Corogne. LAz.

\section{L. lichenoides Fosl.}

$=$ Lithophyllum (Ell. et Sol.) Rosan. - Melobesia Aresch.

Cap d'Alprech, le Tréport, Fécamp. D. - Z. I. Cherbourg. LE JoL. - Iles anglo-normandes. H. V.H. - Roscoff, dragué au large de Duon. J. CH. - Z. M. banc du Château, batterie du Diable. CRN. Biarritz, Guéthary, St. Jean de Luz; San Vicente, au pied des Laminaires ; Gijon. S.

\section{Van Heurckii Heydr.}

\section{= Epilithon Heydr.}

Jersey 1903 et 1904, Ste-Brelade, EP. sur Aglaosphenia attaché à un Halidrys. H. V. H.

Le Dr Henri Van Heurck nous communique, extraits de son Prodrome, la traduction de la diagnose de l'Epilithon Van Heurckii, de M. Heydrich, et les figures qui se rapportent à cette Algue nouvelle.

\section{Epilithon Van Heurckii Heydr. Man.}

Le thalle forme sur Aglaosphenia des petits points roses de 150 à $350 \mu$ de diamètre, pourvus de 2 à 8 lobes inégaux profondément incisés, arrondis, qui ne se réunissent pas et ne croissent pas non plus l'un au-dessus de l'autre, mais qui sont manifestement séparés ou se touchent aux extrémités comme le montre la figure 2. Le thalle n'est pas calcifié, il est fixé par toute sa partie inférieure et composé d'une couche de cellules quadratiques, ayant de 6 à 8 ou de 8 à $8 \mu$ de diamètre et est pourvu de cellules corticales très plates qui sont complètement enfoncées dans les grandes (comparez fig. 3), et qui couvrent la moitié de la grande. Il n’y a pas de cellules-limites.

Les tétraspores, divisées transversalement en deux, se trouvent dans des conceptacles soriformes de 80 à $100 \mu$ de diamètre, qui renferment 7-8 tétrasporanges et ont autant de pores (fig. 2 et 3 ) ; ces conceptacles sont parfaitement sphériques.

De toutes les espèces de Melobesia décrites, il n'y a que le Melobesia membranacea qui se rapproche de l'espèce nouvelle. Mais le Melobesia membranacea est incrusté de chaux, il a des sores ou des conceptacles plus grands et, dans le 
voisinage de ceux-ci, plusieurs couches de cellules et des tétraspores divisées transversalement.

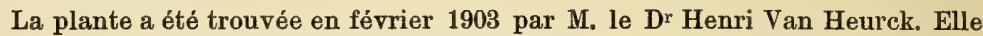
croit sur des tiges d'Aglaosphenia implantées sur Halydris siliquosa rejetées

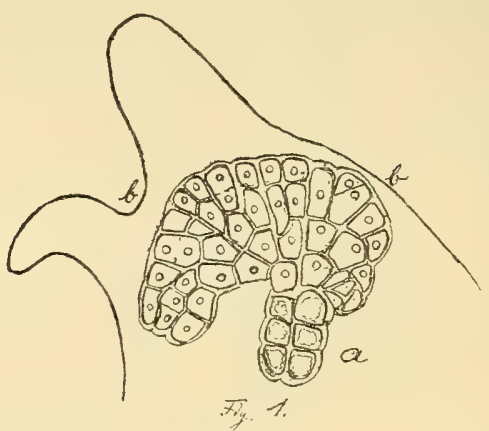
en épave dans la baie de Ste.Brélade à l'île de Jersey. Le même botaniste l'a retrouvée dans des conditions identiques en février 1904. Cette espèce est rare.

\section{Explication des figures.}

Fig. 1. Jeune

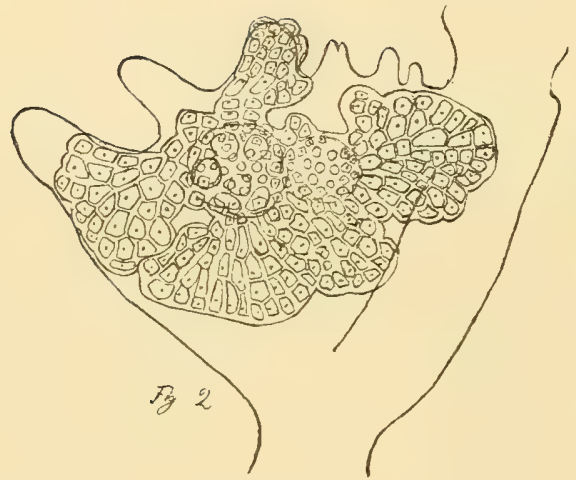
thalle, vu par dessus, sur une tige d'Aglaosphenia.En $a$ on voit les cellules de la spore qui a germé et qui forment un ensem. ble encore nettement reconnais sable. Le thalle s'est développé sur un des côtés des cellules supérieures de la spore. b. b. Aglaos. phenia $\frac{290}{i}$.

Fig. 2. Thalle

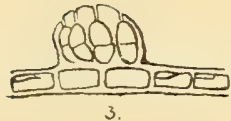

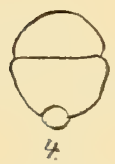

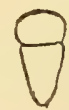

S.

aperȩoit deux sores à tétrasporanges $\frac{164}{4}$.

Fig. 3. Coupe optique longitudinale à travers un sore à quatre tétrasporanges et à quatre pores. On voit les grandes cellules végétatives et les petites cellules corticales. $\frac{290}{1}$.

Fig. 4. Un tétrasporange ancien. $\frac{290}{1}$.

Fig. 5. Tétrasporange jeune. $\frac{290}{1}$. 


\section{Corallina granifera Ell. et Sol.}

= Cor. virgata Zan.

EP. à Ostende. Kx. W. - La Corogne. LAz.

Espèce de l'Adriatique.

\section{C. longifurca Zan.}

= Jania Zan. - Jania Ardiss. p. 460. - Peut-être seulement une forme de Cor. rubens d'après Heydrich. - Espèce douteuse d'après Hauck.

Biarritz, Guéthary, St. Jean de Luz, San Vicente. S.

\section{G. officinalis L.}

EP. Ostende et Nieuport. W. - EP. Blanc.Nez ; Z. M. et Z. I. Gris-Nez, Wimereux, le Portel, le Tréport et toutes les côtes de la Seine inférieure et du Calvados. D. - Z. M. et Z. I. Cherbourg. LE JoL. - Iles anglo-normandes. H. V. H. - Roscoff, entre la ville et l'île Verte, etc. J. Ch. - Le Conquet. Langeron. - Banc du Château. CRN. - Le Croisic. FL. D. - Biarritz, Guéthary, St. Jean de Luz. S. - Santander. Col. - San Vicente, Gijon, Rivadeo, la Corogne. S. - Candas. Laz. CRn.

A. compacta Batt. (=Cor. compacta Crn.) - Fort Mingant.

B. elongata J. Ag. (= Cor. off. typica L.) - Jersey. Melle White. - Batterie du Diable. CRN.

C. mediterranea Aresch. (= Cor. mediterranea Aresch. - Cor. elongatc: (Johnst.) Batt. p. 98). - Iles anglo-normandes H. V. H. Côte S. du Finistère, anse de Déalbors. CRN. - Biarritz, Guéthary, St. Jean de Luz, San Vicente, Gijon, Rivadeo, la Corogne. S. - Très beaux échantillons Z. M. à Guéthary; gazons en touffes compactes de $30 \mathrm{ctm}$. de diamètre à l'île Callot, Z. S. arrosés par l'eau douce à basse mer; petites pelouses rabougries sur les rochers à Callot, Roscoff, Guéthary. J. CH.

D. nana Zan. (= Cor. off. spathulifera incomplètement formé, d'après Heydrich.) - Jersey 1900. H. V. H.

E. procumbens Ell. - EP. côtes belges. Kx. - Algues marines de Cherbourg, No 243. LE JoL.

F. spathulifera Ktz. (=Cor. spathulifera Ktz. - Cor. off. Calvadosii Lamour. - Cor. off. var. d. J. Ag.) - Jersey 1903. H. V. H. - Batterie du Diable. CRN. 
M. Heydrich admet seulement trois formes pour Cor. officinalis $\mathrm{L}$.

Forma typica L. (= Cor. off. L.) - Rameaux arrondis, conceptacles à la pointe des rameaux et latéralement.

Forma spathulifera Ktz. Tab. Phyc. Vol. VIII, pl. 65, fig. 1. (= Cor. compacta Crn.) - Rameaux ordinairement aplatis, conceptacles à la pointe des rameaux et latéralement.

Forma mediterranea Aresch. - Rameaux ordinairement aplatis, conceptacles seulement latéraux.

\section{Gorallina rubens Ell. (Priorité 1755).}

\section{= Jania rubens, Jania adhaerens Lamour.}

EP. entre Heyst et Knocke; Nieuport. Kx. - Fécamp. D. Antifer. Bernard. - Luc, Langrune, Grandcamp. D. - Plage des Bains. Le JoL. - Iles anglo-normandes. H. V. H. - Granville. LeN. - Roscoff, Perkiridic, plage au S. de Ste-Barbe etc. J. CH. - EP. au Conquet. Langeron. - Z. I. environs de Brest. Crn. - Pen-Tir (Crozon). J. Сн. - Le Croisic. Fu. - La Govelle. D. - La Testede-Buch. Chantelat. - Avec anthéridies et cystocarpes, Guéthary. J. Сн. - Sur Algues, Biarritz, Guéthary, St. Jean de Luz. S. Lespinasse. - Gijon, la Corogne. S. - San Vicente, Comillas. Candas. Laz.

A. corniculata Ell. 1755. (= Cor corniculata L. - Jania corniculata Lamour.) - EP à Ostende. W. - EP. à Nieuport. Kx. Grandeamp. D. - Cherbourg. LE JoL. - Iles anglo-normandes. H. V. H. - Roscoff. J. CH. - Environs de Brest. CrN. - La Teste-de-Buch. Chantelat. - Biarritz, Guéthary, St. Jean de Luz, Rivadeo, la Corogne. S.

\section{C. squamata Ell. et Sol.}

EP. à Nieuport. Kx. - Petites-Dalles. Bernard. - Senneville, Fécamp, Yport. D. - Bénouville, Bruneval. Bernard. - Z. I. Hommet, Querqueville. LE JoL. - Iles anglo-normandes. H. V. H. - Roscoff. Sir. - Kaïnou. J. Ch. - Le Conquet. Langeron. Z. I. batterie du Diable, Bertheaume, pointe de Corson. CRN. Belle-Isle. Desmaz. - Le Croisic. Fu. - Z. I. Biarritz, Guéthary, St. Jean de Luz, San Vicente, Gijon, Rivadeo, la Corogne. S.

\section{Amphiroa verruculosa $\mathrm{Ktz}$.}

$=A$. cryptarthrodia Zan. var. verruculosa $\mathrm{Ktz}$.

La Corogne. s. 
Eleutherospora polymorpha (L.) Heydr.

= Lithothamnion L. - Melobesia Harv. - Phymatolithon Fosl. - Lithothamnion purpureum Crn. - Voir aussi plus haut p. 205.

Gris-Nez, Audresselles, Fécamp, Bénouville, Etretat, Antifer, Bruneval, St. Jouin. Bernard. - Port-en-Bessin, Grandcamp. D. Z. M. Cherbourg. LE Jol. - Iles anglo-normandes. H. V. H. Batterie des forts du Diable et du Mingant. CRN. - Le Croisic. FL. - San Vicente, la Corogne. Laz.

\section{Hapalidium callithamnioides Crn.}

= Rhodochorton sp. ? - Melobesia Falk, ?

Rades de Brest et de Camaret, dragué. CRN.

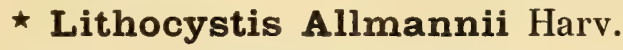

= Lithothamnion corticiforme (Ktz.) Fosl. - Voir une synonymie complète De Toni p. 1759.

Parasite du Chylocladia clavellosa. - Sur Gelidium, Rhodymenia, Furcellaria, Chondrus, ça et là dans l'Atlantique. Kтz.

\section{Hildenbrandia Grouanii J. Ag.} Luz. S.

Z. I. baie de Laninon. CrN. - Biarritz, Guéthary, St. Jean de

\section{H. prototypus Nardo.}

Z. S. Roscoff; de couleur jaune vif, ou pourpre, sur galets. J. CH. - Le Croisic. FL. - Jersey 1903. Iles anglo-normandes. H. V. H. - San Vicente, Gijon, Rivadeo, la Corogne. S.

\section{H. rosea $\mathrm{Ktz}$.} J. Ag.)

$=$ H. rubra Harv. - Haematophlaea Crouanii Crn. (non

Surtout sur silex; Gris-Nez, Audresselles, Wimereux, le Tréport, Petites-Dalles, Fécamp, Yport. D. - Etretat, St. Jouin. Bernard. - Luc, Port-en-Bessin, Grandcamp. D. - Z. M. Cherbourg. Le JoL. - Z. M. environs de Brest. CRN. - Du Pouliguen au Croisic. D.

Réuni par Debray et par Van Heurck à l'espèce précédente. - Ce n'est sans doute que $H$. prototypus d'après Heydrich.

\section{* Erythrodermis Allenii Batt.}

Plymouth, dragué. BatT. 


\section{APPENDICE DES CORALLINÉES}

Le dernier volume du Sylloge Algarum par De Toni vient de paraître. Nous extrayons des Corallinées qui le terminent quelques indications supplémentaires.

Corallina elegans Len. - Portbail (Manche). LEN.

Dermatolithon hapalidioides (Crn.) Fosl. - Sur fragments de porcelaine, Anomies, Balanes; côtes atlantiques de France. CRN. BorN. D. LE JoL.

D. macrocarpum (Rosan.) Fosl. (excl. forma Laminariae). - Sur Phyllophora rubens, Cherbourg. RosanofF.

Lithophyllum Crouanii Fosl. - Sur Laminaria Cloustonii, côtes atlantiques de France. CRN. Le Jol.

Lithoph. subtenellum Fosl. - Guéthary. S.

Lithothamnion australe Fosl. - Côtes atlantiques de France, d'après BORNET. Born.

Lithoth. Bornetii Fosl. - Murs du port militaire de Cherbourg.

De Toni sépare :

Lithoth. coralloides Crn. - Fl. du Finistère p. 151 ; maerl des Bretons. - Brest. CRN. Formes subvalidum, minutum et crassum. et :

Lithoth. calcareum (Ell. et Sol.) Aresch. (= Melobesia Harv.) Côtes atlantiques de France d'après Harvey. Formes d'après Foslie: validum, palmatifidum, squarrulosum, subsimplex, compressum.

Melobesia confervicola (Ktz.) Fosl. (= Hapalidium Aresch.) - Sur Chylocladia clavellosa, Méditerranée et Océan atlantique. De TonI. - Côtes de France?

M. inæquilatera Solms. $=M$. farinos $\alpha$.

I. verrucata Lamour. $=$ M. pustulata.

Hapalidium callithamnioides $\mathrm{Crn} .=M$. callithamnioides. 


\section{FLORULE DE TATIHOU}

Pendant l'impression de ce volume, M. Malard a bien voulu me communiquer le manuscrit volumineux de ses observations algologiques autour de Tatihou; j'espère que M. Malard se décidera à les publier un jour in extenso ; elles formeraient une excellente florule de la localité.

Je dois aujourd'hui me borner à en extraire - avec l'autorisation de l'auteur - un certain nombre de notes inédites intéressantes, et les stations d'espèces rares ou nouvelles destinées à compléter ma Liste. Une quantité d'autres espèces, indiquées déjà par Le Jolis à Cherbourg et environs, ont été retrouvées aux environs de Tatihou; mais de même que pour les îles anglo-normandes de Van Heurck, il n'est pas possible de donner ici le détail de toutes ces stations.

Acrochætium entophyticum Batt. - Doit exister à Gatteville où l'on remarque souvent sur Dasya un feutrage de petites filaments.

A. endozoicum Batt. - Pas rare dans Alcyonidium gelatinosum dragué au petit Nord l'automne et l'hiver. Beaucoup plus rare dans les Alcyonidium de la côte.

Aglaozonia reptans Ktz. - Commun dans les cours des parcs aux huitres, surtout sur les tubes des Sabelles. - Avec les formes jeunes de Cutleria naissant de son thalle, sur des cailloux au-dessous de la prairie de Zostères entre l'Ilet et la Dent.

Antithamnion cruciatum Näg. - Doit exister sur toute la côte N. du Cotentin, mais très rare. - Gatteville. Pelvet. - Cherbourg. Le Jol. Thur. - Cosqueville EP. Malard.

Ascophyllum nodosum Le Jol. - Atteint 2 mètres de longueur, avec flotteurs gros comme un wuf de poule, aux îles St. Marcouf.

Bangia elegans Chauv. - Tatihou, la Hougue, vanne de la saline près de Gatteville.

B. Le Jolisii De Not. - Estacade en bois de l'anse de Roubaril (Gatteville). 
Bangia versicolor Ktz. - Pas rare entre les Porphyra vulgaris linearis sur les grosses têtes des rochers exposées au choc des vagues, à Réville et Réthoville. Au premier printemps.

Bonnemaisonia asparagoides Ag. - Tatihou 27 août 1863. Thur. - EP. anse de Gatteville en août, pas rare, ordinairement bien fructifié.

Bostrychia scorpioides Mont. - Très commun à la base des Atriplex, anse du Cul-de-Loup, à la Hougue.

Brongniartella byssoides Schm. - Dragué au petit Nord, parfois EP. dans l'anse de Gatteville, trouvé en place par ThURET le 15 août 1863.

Calliblepharis jubata Ktz. - Commun, très variable de forme, sur diverses Algues dans les flaques à Gatteville, St. Marcouf, Ile du large etc.

Callithamnion corymbosum Lyngb. - Les formes secundatum et amphicarpum ont été recueillies dans les régions vaseuses, en certains points des parcs aux huîtres, à la pointe de l'Epée très abondamment, par Pelvet, Thur. Born. Le Jol. Toutes les formes existent dans l'anse de Gatteville.

C. polyspermum Ag. - Sur les Fucus, spécialement dans les environs des rochers des Anes, île de Tatihou, mars-avril.

C. tetragonum Ag. - Sur rochers et Fucus, Z. I. anse de Gatteville; sur Rhodymenia au N. W. de la balise, à Tatihou entre le Cavat et San-Hougue.

Callocolax neglectus Schm. - Gatteville.

Capsosiphon aureolus Gobi. -- A l'entrée d'un ruisseau se jetant dans le Cul-du-Loup près de Morsalines, sur de petites pierres plates. Déjà trouvé là en 1863 par Thuret.

Carpomitra Cabreræ Ktz. - Sur place (?) dans l'anse de Gatteville après les coups de vent, en été. La remarque de M. Bornet sur le Sporochnus (voir plus loin) est applicable au Carpomitra. 
Ceramium acanthonotum Carm. -- Rochers couverts de Balanes, où poussent les Nemalion, à l'entrée de l'anse de Gatteville.

C. circinatum J. Ag. - Recueilli en août 1850 à Gatteville; y existe toujours.

C. Deslongehampsii Chauv. - Habitat particulier en Jedans des jetées au N. sous les Fucus Z. M. avec Cladophora rupestris, aux endroits où l'eau contenue dans les murailles s'écoule peu à peu à basse mer.

C. strictum Grev, et Harv. - Z. I. en mai, très rare. La variété zostericola est au contraire extraordinairement commune: Tatihou, la Hougue, entre Réville et le Drauguet, près du phare de Gatteville etc.

Chretopteris plumosa Ktz. - Très douteuse dans les dragages au petit Nord, jamais à la côte jusqu'ici. En tous cas, si elle existe, est extrêmement rare.

Champia parvula J. Ag. - Parois des rochers à basse mer, Gatteville, anse du phare, côté du large. Automne, pas commun. Le 15 août 1863 à la Hougue. THur.

Chantransia Daviesii Thur. - Commun à très basse mer sur Rhodymenia palmata ; Gatteville, île de Tatihou au N., le Founet, le Cavat, San Hougue.

Chilionema Nathaliæe Sauv. - Sur Laminaires, à l'entrée d'un petit cours des parcs aux huîtres parallèle au Rhun, au S.

Chondria cærulescens Falk. - Forme une véritable prairie dans la vase qui entoure la balise près de la pointe de l'Epée à la Hougue.

Ch. dasyphylla Ag. - Particulièrement abondant à l'ìle St. Marcouf, dans le cours entre la balise et le petit port, Z. I.

Ch. crispus Lyngb. - La var. angustifrons est commune à Gatteville, et la var. latifrons à la Hougue.

Choreocolax Polysiphoniæ Reinsch. - Ile St. Marcouf. 
Chylocladia clavellosa Grev. var. pyramidalis. - Sur Patelles et Balanes, îlot rocheux à l'entrée du goulet de l'anse de Gatteville.

Cladophora Balliana Harv. - Dans l'herbier du Laboratoire existe un exemplaire qui porte le nom de Cl. Rudolphiana (Ag.) Harv. La même plante se rencontre à Gatteville; M. Malard la rapporte à $\mathrm{Cl}$. Balliana.

Cl. expansa Ktz. - Flaques des rochers vaseux à la pointe de l'Epée à la Hougue, avec sa var. glowierata.

Cl. hirta Ktz. - Cap Lévy et Cosqueville. Rare.

Codium bursa Ag. - Très commun sur la côte W. à partir de Portbail ; EP. - Iles Chausey.

C. tomentosum Stackh. - Présente à St. Marcouf une forme très rameuse, à ramuscules courts et dressés, qui hérissent tous les rameaux principaux.

Colacolepis incrustans Schm. - Sur Phyllophora rubens, Tatihou. Ce Phyllophora est souvent rejeté à la côte l'hiver; pas rare en place à l'anse de Gatteville.

Corallina officinalis L. - Très commun et très variable de forme. Des coussinets, que M. Malard considère comme une forme réduite, se trouvent souvent sur les Patelles, près de la balise de Barfleur; est-ce le Cor. compacta Crn.? - La plus commune est la var. nana; la diagnose de Zanardini doit correspondre à cette dernière forme très abondante à Gatteville.

Cordylecladia erecta J. Ag. - Près de la pointe de Drauguet, dans les flaques sablonneuses du N. pendant l'hiver; très rare. - Près de Gatteville, dans les flaques, anse du Phare.

Crouania attenuata J. Ag. - Très rare sur diverses Algues, Ceramium, Cladostephus, au goulet de l'anse de Gatteville près du Phare.

Cruoria pellita Lyngb. - Recueilli le 30 août 1863 par ThureT à la pointe de la Hougue; Z. I. sur la Dent de Tatihou. 
Cruoria purpurea Crn. - M. Malard pense que cette espèce existe, pas très rare, sur Lithothamnion coralloides compressum Heydr. du grand Nord, fond de pêche au large de Tatihou. Mais il n'a pu trouver d'assez beaux exemplaires fructifiés pour en être sûr; en tous cas, l'espèce serait très voisine.

Cutleria multifida Grev. - Beaux exemplaires sur les pierres dans les flaques creusées au milieu des Zostères, au S. de l'île de Tatihou, entre l'Ilet et la Dent. Z. I.

Cystoclonium purpurascens Ktz. var. cirrosum. - Très beaux échantillons à Gatteville.

Dasya ocellata Harv. - Sur les rochers à Corallines dissimulés dans les herbiers de Zostères à la Roche-jolie, entre Morsalines et Aumeville.

Delesseria sinnosa Lamour. - Commun EP. sur stipes do Laminaria Cloustonii, anse de Gatteville; plus rare au N. de l'île ; rare en place.

? Dermatolithon hapalidioides var. confine. - Sur les Corallines, île rocheuse dans le goulet de l'anse de Gatteville.

D. macrocarpum Rosan. - Avec Melobesia membranacea sur stipes de Laminaria Cloustonii.

Dermocarpa Leibleiniæ Born. - Sur les filaments très sales de Lyngbya aestuarii au Cul-du-Loup.

Dudresnaya coccinea Crn. - En août et septembre, EP. très commun. Plus rare en place: le Cavat (Tatihou), la Bécue. Déjà recueilli par PeLveT dans ces localités.

Ectocarpus Battersii Born. - La plante d'Arromanches qui croît sur les Taonia et qui a été distribuée sous le No 211 des Algues marines d'Hohenacker, se retrouve sur les vieux pieds de Padina Pavonia poussant près de la balise au-dessous de la Bécue. Les Taonia des parcs sont généralement très propres et ne portent pas d'Ectocarpus.

E. confervoides Le Jol. - Commun sur diverses Algues, sur toute la côte entre St. Vaast et la pointe de la Hougue. 
Ectocarpus Crouanii Thur. - Pas rare au printemps dans les flaques, près du phare de Gatteville à l'E. sur Scytosiphon lomentarius.

E. distortus Carm. - Le 8 avril 1894 dans les huîtrières de Tatihou. Malard et Gomont.

E. fasciculatus Harv. - Pas rare à Gatteville, Z. I. sur Himanthalia et Laminaria, surtout au automne.

Une forme réduite, pas rare sur Fucus serratus. Est-ce la forme abbreviatus? MALARD et GoMonT.

La forme draparnaldioides est beaucoup plus rare. Sur Saccorhiza, THURET 1850.

E. insignis Crn. - Sur les Zostères et les tubes des Sabelles, bancs de Zostères sous les pares de Tatihou, 29 août 1863. Thur.

E. Sandrianus Zan. - Dans les huîtrières de St. Vaast, 15 juill. 1863. THUR.

E. terminalis Ktz. - Trouvé à Tatihou par Kuckuck, mêlé à $E$. virescens, sur les rochers à l'entrée du cours, après les deux parcs qui suivent le pare du Muséum, et en descendant du N. au S.

Endodictyon infestians Gran. - Petites taches brunâtres un peu allongées sur Alcyonidium gelatinosum de la côte.

Epicladia Flustræ Rke. - Sur les Alcyonidium gelatinosum de la côte. Au printemps dans les loges des Sertulariens, principalement Sertularella pumila, qui sont alors presque complètement colorés en vert. Très commun. rubrum.

Erythrotrichia ceramicola Aresch. - Commun sur Ceramium

Gelidium corneum Lamour. - Anse de Gatteville, avec une variété presque infinie de formes.

G. latifolium Born. - Sous les gros rochers, et dans les fentes profondes.

Giffordia Lebelii Batt. - Commun sur Cystoseira ericoides à Tatihou. Kuckuck. 
Giffordia secunda Batt. - Sur Polysiphonia à Tatihou. Thưr. 1866.

Gomontia polyrhiza Born. et Fl. - Assez commun dans coquilles de Tapes decussatus.

Gracilaria confervoides Grev. - Forme allongée, très peu rameuse, dans les cours d'eau sur le sable à basse mer, au N. W. du banc de l'Alouette.

Griffithsia barbata Ag. - La Hougue, 15 août 1863, 1 et 11 août 1866 ; Tatihou 1866. Très rare.

Gymnogongrus norvegieus J. Ag. - La Hougue et Gatteville; rare en dehors de ces deux localités.

Halopithys pinastroides Ktz. - Commun dans toutes les flaques, île de Tatihou, Gatteville etc. Rare en échantillons propres.

Halopteris filicina Ktz. - Rejeté à la côte sur les stipes des Laminaires dans l'anse du phare de Gatteville.

Halosphæra viridis Schm. - Dans des pêches au filet fin faites au large de Gatteville. Extrêmement rare.

Helminthocladia purpurea J. Ag. - Anse de Gatteville, très rare. Pelvet. Thuret.

Hydrocoleum Lyngbyaceum Ktz. - Les deux variétés se rencontrent, la première sur Cladostephus et la seconde (rupestre) sur les murs des quais. - Ile St. Marcouf.

Hyella caspitosa Born. et Fl. -- Dans le test de Tapes decussatus, où il forme des taches grisâtres, petites, nombreuses. Vieux pares de la Couleige.

Hypnea musciformis Lamour. - Trouvé deux fois seulement en place dans les vieux pares à huîtres de St. Vaast.

Hypoglossum Woodwardii Ktz. - La var. angustifolium semble la plus commune. - On trouve sous la Hougue, dans l'anse vaseuse qui précède la balise, sur des rochers à basse mer, la forme glomeratum souvent décolorée ot verte; elle passe à la forme 
crispum. - On trouve cette dernière très belle à Gatteville. - La forme arborescens est rare : une seule fois à Réville dans une anse vaseuse, échantillon unique.

Isactis plana Thur. - Très commun sur vieilles coquilles d'huîtres et petits cailloux des vieux pares, en taches orbiculaires ou confluentes de quelques centimètres. A St. Marcouf, île du large, recouvre galets et pierres plates en plages de 15 à 20 cent et davantage.

Laminaria saccharina Lamour. - Suivant Kuckuck, la grande Laminaire à bords plissés et tuyautés n'est pas $L$. saccharina, mais une espèce différente, plus méridionale. Le vrai $L$. saccharina est à bords plats, dans le plan de la lame médiane.

Laurencia cespitosa Lamour. - Très commun sur les rochers à Balanes, près du phare de Gatteville, entre l'anse du phare et celle de Roubaril, Z. I.

Lithocystis Almannii Harv. - M. Malard l'a trouvé tel qu'il est figuré dans Harvey, sur des colonies de Bryozoaires (Scrupocellaria et Crisia). Vu au microscope, c'est absolument la figure 2 de la planche CLXVI d'Harvey. Est-ce avec raison que Crouan l'assimile à Hapalidium confervoides, dans Florule p. 149 ?

Lithothamnion calcareum Aresch. - Formes compressum, subvalidum et flabelligerum : au petit Nord et au grand Nord. Formes mamillosum et crassum : huîtrière de Happetout.

Lophosiphonia obscura Falk. (Harvey Pl. 102 A.) - Dans l'herbier de M. Malard, un échantillon de $P$. obscura Harv. donné par Le Jolis, No 1431 de son herbier, Cherbourg 4. 10. 1858; "rarissime, semel reperta, „, sur Balanes, dans une flaque Z. M.

Harvey nous apprend que cette espèce a été découverte par Melle White en 1846, dans les souches du P. fibrata à Jersey. M. Cresswell en a trouvé une station abondante près de Sidmouth. Harvey ne parle pas du P. opaca.

D'après Le Jolis (Liste p. 145), le P. obscura J. Ag. est le No 229 des Algues de l'Ouest de Lloyd, et le No 303 des Algues du Finistère de Crouan. Le Jolis ne parle pas de P. opaca (Ag.) Zan.

Mais Crouan désigne lui-même son $\mathrm{N}^{0} 303$ (Florule p. 158) sous le nom de $P$. opaca $(\mathrm{Ag})$ Zan. var. ascendens. 
Hauck et De Toni décrivent comme espèces bien distinctes $P$. obscura (= Lophosiphonia) et $P$. opaca. Voilà encore une question à élucider.

Lyngbya ærnginosa Ag. - La forme fusca a été trouvée par Thuret à St. Vaast-la-Hougue. On en rencontre les diverses variétés au Cul-du-Loup.

L. æstuarii Lieb. - Très pur et avec ses différentes formes, entre autres la forme spectabilis, dans une mare saumâtre, à demi desséchée, en dedans de la digue $\mathrm{N}$. de l'île Tatihou.

L. semiplena J. Ag. - Z. S. au N. de l'île de Tatihou, dans une flaque; derrière l'Ilet et sous le fort de la Hougue. Il débute par la forme viridis, puis prend peu à peu la forme sordida, pour se détacher en croûtes qui se lavent au flux et reflux, et redeviennent vertes comme des lambeaux de drap de billard vers la fin de septembre.

Mastigocoleus testarum Lagerh. - Taches orbiculaires puis confluentes, gris bleuâtre ou violacé, dans coquilles d'huîtres mortes, pares de la Toquaise.

Melobesia Le Jolisii Rosan. - Avec M. farinosa sur les Zostères. Le premier est le plus commun à St. Vaast, le second à Gatteville.

M. membranacea Lamour. - Sur vieilles frondes de Rhodymenia et sur Cystoseira; Gatteville, le Cavat.

M. pustulata Lamour. - Commun sur Chondrus crispus et Phyllophora rubens, mare de San Hougue, Gatteville.

Microcladia glandulosa Grev. - Ne se trouve jamais sur la côte E. du Cotentin. Très exceptionnellement EP. après les tempêtes au printemps ou à l'automne, dans les baies largement ouvertes au N. comme Réthoville ou Cosqueville.

Microcoleus Chthonoplastes Thur. - Au lieu dit la Saline (route de Barfleur à Gatteville). — Anse du Cul-du-Loup à St. Vaast. 
Myrionema papillosum Sauv. - Sur Laminaria saccharina à l'entrée du petit cours conduisant au parc du Laboratoire. S.

Naccaria Wigghii Endl. - Dans l'anse de Gatteville près du phare, 11 août 1866. Thur. et BonN. On l'y rencontre encore parfois après les tempêtes d'été.

Nemalion lubricum Duby. et var. dichotomum. - Sur les Patelles et les rochers à Balanes du phare de Gatteville.

Nitophyllum Hilliæ Grev. - Sur les gros rochers près de la balise de Gatteville. d’Ovit.

N. punctatnm Grev. - Jeté à la côte : île Tatihou, mare

N. uncinatum J. Ag. - Pointe de Barfleur près de la balise. Très rare. Saire.

Oscillatoria amphibia Ag. - Près des piles du pont de la

0. chalybea Mertens. - A Morsalines, dans la vase des petits fossés se rendant à la mer; près du pont de la Saire.

0. tenuis Ag. - Avec O. chalybea.

Ostreobium Quekettii Born. et Fl. - Pas rare dans coquilles d'Anomia Ephippium des pares de la Couleige sous le Laboratoire.

Phæocystis Ponchetii Lagerh. - Extraordinairement commun dans les pêches au filet fin, en janvier et février, jusqu'en juin.

Voir : Malard, Variations de la Faune et de la Flore de la baie de la Hougue, dans Bull. du Muséum d'hist. Nat. 1902, № 3.

Phormidium autumnale Gom. - Sur les murs des quais, à la limite de la haute mer.

Phyllophora membranifolia J. Ag. - Très commun dans les petites flaques entre les gros rochers, dans les fentes peu éclairées et sous les roches surplombantes, au Cavat, à San-Hougue, dans l'anse de Gatteville. 
J'ai observé, écrit M. Malard, ce que je crois être un Colacolepis (d'après M. Gomont?) sur Phyllophora membranifolia.

Phyllophora palmettoides J. Ag. - Z. I. sur les rochers sablon. neux de la Dent (Tatihou); à l'entrée de l'anse de Gatteville. Au premier printemps.

Ph. rubens Grev. - Souvent EP. en hiver, bons échantillons avec fructifications. En place, anse de Gatteville.

Polysiphonia Brodiæi Grev. - Présente deux formes, l'une correspondant au $\mathrm{N}^{\circ} 14$ des Algues marines de Cherbourg de Le Jolis ; du commencement du printemps jusqu'en avril. C'est la forme jeune, probablement correspondant à la var. densa de Holm. et Batt. ; elle est continue. Dans le cours de l'été, vers la mi-mai, les pinceaux s'écartent et la tige principale se dénude en passant par une forme moyenne figurée par Harvey, pour aboutir à une forme tout à fait discontinue à l'automne.

En résumé, le même individu, d'après l'âge, prend successivement les différentes formes décrites par les auteurs. Il en est de même pour beaucoup d'Algues.

P. elongella Harv. - Près du Cavat, balise au-dessous de la Porte-aux-dames, sur les cailloux disséminés sur le sable très vaseux. Rare.

P. fotidissima Cockx. - Trouvé en septembre 1850 aux environs de la Bécue, et le 17 août 1863 dans les huîtrières par Thuret. Espèce de l'arrière-saison, peu commune.

P. furcellata Harv. - Trouvé le 15 août 1863 à la Hougue par Thunet, entre l'église et la tour. - EP. dans les huîtrières au N. du Rhun, dans les pares etc.

P. pulvinata J. Ag. - La synonymie est embrouillée, les auteurs ne sont pas d'accord.

D'abord il faut écarter le $P$. pulvinata Ktz. qui est une espèce de l'Adriatique (Hauck, p. 219).

Le $P$. pulvinata J. Ag. signalé par Le Jolis dans sa Liste des Algues de Cherbourg figure dans l'herbier de M. Malard en deux échantillons de Le Jolis : No 329, Cherbourg 30.1.1854, et No 1593, Cherbourg 8.3.1860. Mais ces deux échantillons se rapportent cer- 
tainement au P. sertularioides (Grat.) J. Ag., comme le témoigne un autre exemplaire recueilli par Thuret 6.9.1853 et signé de lui.

La synonymie de Crouan (p. 156) serait donc exacte, et l'on aurait $P$. pulvinata $=P$. sert , larioides; Lloyd Algues de l'Ouest 235 ; Crouan No 288 ; Harvey pl. 102 B.

Voir De Toni, P. sertularioides p. 870 ot P. pulvinata J. Ag. p. 895. ci-dessus.

Polysiphonia sertularioides J. Ag. - Voir P. pulvinata

P. rhunensis Thur. [Thuret, études phycologiques]. - Cette espèce a été découverte par ThuRet en 1850 dans le Rhun, passage à basse mer entre la terre et l'île Tatihou. On le trouve dans les flaques au $\mathrm{N}$. de ce passage.

P. urceolata Grev. var. comosa. - Sur le Rhun, dans les huîtrières, à Tatihou. Thur. 1863.

Pteridium alatum J. Ag. - Deux habitats très différents; $1^{0}$ avec les Ptilota, sous les rochers à mi-marée, par exemple à l'E. de la mare d'Ovit ; $2^{\circ}$ sur les stipes de Laminaria Cloustonii, où il prend une forme plus grande, plus élancée; commun après les tempêtes dans l'anse de Gatteville.

Pt. angustissimum J. Ag. - Trouvé une seule fois sur un stipe de Laminaria Cloustonii.

Pterocladia capillacea Born. et Thur. - Très commun et très beau dans l'anse de Gatteville, contre les rochers à pic vers l'E.

Pterosiphonia pennata Falk. - Existe certainement sur les côtes de la Manche. EP. à Quineville.

Pt. parasitica Falk. - Côte W. du Cotentin, anse d'Escalgrain, anse de Nauville, EP. - Côte E.? - Jamais observé sur la côte N.

Punctaria cespitosa J. Ag. - Sur les pierres dans le cours sablonneux descendant du milieu du Rhun vers la jetée de St. Vaast. Assez commun au printemps. 
Rhodochorton membranaceum Magnus. - Très commun dans Sertularia abietina, et Hydrallemannia falcata du petit Nord.

Rhodomela. - Harvey considère comme espèces distinctes Rh. lycopodioides et Rh. subfusca; il donne deux planches différentes. - D'après M. Malard, le $R h$. subfusca est commun dans les flaques sablonneuses, Z. M. au printemps, surtout à la mare d'Ovit. Le Rh. lycopodioides n'est pas connu.

Rhodophyllis appendiculata J. Ag. - Très rare et seulement en EP. d'après LE JoL.

Rivularia atra Roth. - Plusieurs variétés bien tranchées :

a. En tabourets orbiculaires sur les rochers ou les Fucus, formant de petites masses d'un noir brillant, quelquefois confluentes et devenant alors d'un jaune verdâtre ou brunâtre foncé. Sur les Balanes elles restent généralement punctiformes.

$\beta$. Sur les Lichina au sommet de la zone des Balanes, affectant alors la forme de petites sphères, de guttules ou de masses amygdaloïdes réunissant par une croûte ininterrompue les Lichina.

$\gamma$. Formant avec le Calothrix scopulorum des sortes de cornes empâtant les extrémités du Fucus platycarpus. Les masses sont alors diffluentes comme de la gelée.

R. bullata Berk. - Z. M. sur les grosses têtes des rochers exposées au choc des vagues; chaque année sur les mêmes emplacements de la fin d'août jusqu'à octobre. Nord de l'île de Tatihou, Gatteville.

Schizothrix vaginata Gom. - A Gatteville sur les Balanes, derrière le vieux Phare.

Seirospora flaccida Ktz. (= Call. versicolor Drap. ?) - Très abondant à St. Vaast, à Tatihou, à la Hougue.

S. Griffithsiana Harv. - Trouvé une fois par Thur. sur Arthrocladia villosa, EP. côte N. du Cotentin. Excessivement rare.

Spermatochnus paradoxus Roth. - La Hougue, dans les rochers auprès de la jetée de St. Vaast; dans les huîtrières. 
Spermothamnion Turneri Aresch. et var. repens. - Sur Halidrys, Codium tomentosum et diverses Algues, Z. I. près des pointes de Drauguet, Réville, Tatihou (la Dent), mare d'Ovit.

Sphacelaria bipinnata Sauv. - Sur Halidrys, mare d'Ovit; avec Melobesia sur Aglaosphenia pluma à Réville.

Sph. Hystrix Suhr. - Sur Cystoseira ericoides, entrée du grand cours des parcs de Tatihou.

Sphondylothamnion multifidum Näg. var. piliferum. - Sur les rochers près de la balise de la pointe de l’Épée.

Spirulina versicolor Cohn. - Sur Flustra foliacea rejeté au rivage après les tempêtes d'hiver.

Sporochnus pedunculatus Ag. - Anse de Gatteville. Huîtriêres de St. Vaast, ayant l'apparence de s'y trouver fixé. "C'est une plante d'été, écrit M. Bornet; on ne la rencontre pas in situ natali mais seulement jetée à la côte. Comme elle arrive en état de fraîcheur parfaite, presque toujours fixée à un petit galet ou à une coquille qui lui sert de lest, elle se tient debout et on la croirait née sur la place où on la trouve, .

Stenogramma interruptum Ag. - Sur les Pecten dragués au large. Cherbourg. LE JoL.

Streblonema Valiantei De Toni. - Avec Sphacelaria Hystrix, voir ci-dessus.

Str. volubile Thur. - Sur Dudresnaya au Cavat, à la pointe du Founet, à San-Hougue, sur l'île Tatihou ; EP. près du Cavat. Vire.

Vancheria synandra Wor. - Marais de l'embouchure de la

V. dichotoma Ag. - Vers l'embouchure de la Vire.

V. litorea Hoffm. et Ag. - Sur la vase à demi desséchée de l'embouchure de la Sinope à Quineville. 
Vaucheria Thuretii Wor. - Forme de véritables prairies d'un vert sombre ou sale au-dessus des prairies de Zostère sur la vase à demi desséchée juste devant le Laboratoire de Tatihou.

Wildemania laciniata De Toni. - Avec ses variétés linearis et umbilicalis croît sur les cailloux à haute mer, chemin de l'île Tatihou à l'Ilet, Port-aux-Dames à la Hougue, rochers du haut du Rhun, sous le feu rouge de St. Vaast à l'entrée du port. C'est l'espèce figurée par Harvey. Elle débute toujours par une forme linéaire, de préférence sur le rocher; la var. linearis atteint 50 cent. sur 2 cent. de largeur environ. Sa couleur passe du jaune verdâtre ambré au jaune verdâtre olive foncé, puis au bleu violacé et enfin au brun rougeâtre.

On trouve encore trois variétés :

A. Sur les grosses têtes de rochers au niveau de la haute mer une variété linéaire qui reste toujours petite, n'excédant que rarement 10 à 15 cent. de longueur, ne changeant jamais sa forme linéaire en celle d'un thalle membraneux lacinié, enfin ne descendant pas au-delà de quelques pieds en profondeur, tandis que la forme précédente plonge à quelques mètres. En outre elle habite toujours en masse serrée, jamais en individus plus ou moins isolés.

B. Une variété qui semble être une forme rabougrie de l'espèce, d'une couleur bleu-lilas, très mince, petite, sur les têtes de rochers immergés à quelques mètres de profondeur, prenant dès le premier âge la forme laciniée. Grosse Roche etc.

C. Enfin la forme leucosticta de Thuret, bien distincte non seulement par le caractère des taches, mais surtout par le genre de vie et le développement. Toujours l'espèce commence et se conserve longtemps en un thalle arrondi ou ovale qui se maintient jusqu'à la fin; il devient seulement réniforme par suite de son hile, ou point d'attache. Toujours fixée sur une autre Algue. Croissant toujours à une certaine profondeur, plusieurs mètres. 


\section{ERRATA}

La fiche du Bryopsis accidentellement déplacée est tombée p. 63. Il faut la restituer à la p. 73 .

Endoderma leptochaete p. 63 doit avoir l'astérique des espèces à rechercher.

Polysiphonia macrocarpa p. 168 doit être fondu avec P. sertularioides p. 169.

Callithamnion Dudresnayi p. 183 doit être fondu dans Call. acrospermum p. 182.

P. 103 : Phloeospora et non Phaeospora.

P. 204 : il faut lire Melobesia inaequilatera Solms.

Les noms d'espèces terminés en cola, tels que rupicola, corallicola, doivent rester invariables: ce sont des substantifs comme incola.

Le grec oıs a été traduit d'abord par $a$ o et l'on écrivait autrefois homceopathe; aujourd'hui on écrit homéopathe. Dans les mots scientifiques, de création récente, certains auteurs adoptent ceo [Gloeocystis, Glocothece], d'autres oio [Gloiopeltis, Mesogloia]. Il est à désirer que ces differentes graphiques soient unifiées en dans les mots latins, en éo dans les mots français. 


\section{RÉPERTOIRE ALPHABÉTIQUE GÉNÉRAL}

\section{DES NOMS DE GENRES, ESPĖCES ET VARIÉTÉS}

abbreviatum Callithamnion . . 178

Ectocarpus 103, 104, 218

Abies-marina Cystoseira . . 120 abrotanifolia Cystoseira . . 120 acanthonotum Ceramium . . 191, 215 acanthophorus Ectocarpus . . 103 acicularis Gigartina . . . 138

Halymenia . . . 196 Sphærococcus . . . 138

Acinetospora. . . . . . 112

Acrochaete . . . . . 63

Acrochætium 130, 131, 132, 182, 213

acrospermum Callithamnion. . 182.

Actinococcus . . . . . . 143

aculeata Desmarestia . . . . 91

" Polysiphonia. . . 171

"Sporochnus . . . . 91

aculeifera Polysiphonia . . . 171

acuta Dictyota . . . . . . 123

Fucus . . . . . 118

acutirostris Microcoleus . . . 42

adhærens Codium . . . . 75, 76

Cruoria . . . 201

Jania . . . . 210

Nemalion . . . 201

adnata Glœocystis . . . . . 51

adriatica Bryopsis . . . . . 73

" Lithoderma . . . 80

\begin{tabular}{llll} 
Palmella . . . . . & $\cdot$ & $\cdot$ & \\
\hline 2
\end{tabular}

Stictyosiphon . . 93

adspersa Cutleria . . . 78, 79

Padina . . . 78

Zonaria . . . 78

adunca Polysiphonia . . . . 175

æcidioides Ectocarpus . . . . 109

Agagropila Sphacelaria . . . 99

æequalis Chondrus . . . . . 138

Phæostroma . . . 109

Streblonema . . . 109 ærea Chaetomorpha . . . . 65

æruginea Calothrix. . . . . 43

Gloocapsa . . . 31

"Leibleinia . . . . 43

æruginosa Lyngbya . . . 39, 221

æstivale Gastroclonium . . 152

Halopteris. . . . . 101

" Stypocaulon . . . 102

æstuarii Lyngbya . . . . 39, 221

affine Callithamnion . . . 182, 183

"Ceramium. . . . . 182

" Polysiphonia. . . . 168

Agardhia . . . . . . . 76

Agardhii Calothrix . . . . . 39

" Lyngbya . . . . . 39

Agardhianum Ceramium . . . 192

Enteromorpha. . $\quad 56$

Hutchinsia . . 164

Polysiphonia . . 165

aggregatus Actinococeus . . . 143

Sphærococcus . . 142

Aglaophyllum . . . 156, 157, 158

Aglaozonia . . . 78, 79, 213

Ahnfeltia . . . . . . 142

Alaria . . . . . . . 82

alatum Pteridium . . . . 160, 224

angustissima Delesseria. 160

albida Cladophora . . . . $\quad$. 67

refracta Cladophora . . 71

albus Choreocolax . . . . . 134

Alcyonii Oscillatoria . . . . 36

Alcyonium . . . . . . 76

Allenii Erythrodermis . . . . 211

alliaceum Nitophyllum. . . . 156

Allmannii Lithocystis . . . 211, 220

allochroa Polysiphonia. . . 171, 172

Alsidium . . . . . . . . 161

alternans Fucus. . . . . . 118

ambiguum Herponema. . . . 113 
amethystea Leibleinia. . . . 44 Pleurocapsa . . . 33

" Porphyra . . . . 128

"Wildemania . . . 128

amorpha Oscillatoria . . . . 37

amphibius Ectocarpus . . . . 107

Oscillatoria. . . 35, 222

amphicarpum Callithamnion 183, 214

, Sphacelaria . . 98

Amphiroa . . . . . . . $\mathbf{2 1 0}$

Amphithrix . . . . . . 43, 45

Anabaena . . . . . . . . 49

Anacystis . . . . . . . . 32

anguiformis Microcoleus . . . 42

angulosa Polysiphonia. . . . 172

angusta Gigartina . . . . . 140

Laurencia . . . . 162

angustifolius Fucus. . . . 117, 118

Hypoglossum . 159, 219

Pteridium . . . 160

angustifrons Chondrus. . . 138, 215

Plocamium . . . 155

angustior Desmarestia. . . . 92

angustissima Delesseria . . . 160

Dictyota . . . 124

Gracilaria * . 149

Hypoglossum . . 160

Polysiphonia . . 165

Pteridium . . 160, 224

Antithamnion. . . . 189, 213

antliaria Oscillatoria . . . 38

antliarum Phormidium. . . . 37

Aphanothece . . . . . . 32

apiculata Spyridia . . . . . 191

Apoglossum . . . . . . . 159

appendiculata Rhodophyllis . 146, 225

Vaucheria . . 74

arachnoideum Ceramium . . . 195

Ectocarpus. . . 106

patentissimum Cera. mium. . . 192, 195

arborescens Ceramium. . . . 191

Hypoglossum . 159, 220

arbuscula Bryopsis. . . . . 73

Callithamnion . . 173, 182

Dasya. . . . . . 173

Phlebothamnion . . 182

" mucilaginosa Dasya . 173

arcta Cladophora . . . . . 67

" Ectocarpus . . . . 108, 107 arctica Bangia . . . . . . 127

arenaria Polysiphonia . . . 169

arenosum Rhizoclonium . . . 66

Areschougia . . . . . . . 90

Areschougii Elachista . . . 89

armata Gracilaria . . . . . 147

, Polysiphonia . . . 170

armorica Cruoriella. . . . . 201

Nodularia. . . . . 50

Arthrocladia . . . . . . . 93

Arthrospira . . . . . . . 38

articulata Chondria. . . .152, 164

" Chylocladia. . . 152

" Lomentaria . . . . 152

ascendens Polysiphonia . . 169, 220

Ascocyclus. . . . . . 111, 113

Ascophyllum . . . . 118. 213

asparagoides . . . . . . 160, 214

Asperococcus . . . . . 94, 96

atlantica Bryopsis . . . . . 73

Symploca. . . . 41

atomaria Taonia. . . . . 124

atra Rivularia . . . . . 46, 225

Atractophora . . . . . . . 134

atro-purpurea Bangia . . . . 128

" Peyssonnelia . . 201

" Physactis . . . 46

" Porphyra . . . 128

atro-rubescens Polysiphonia . . 164

attenuata Carmichælia. . . . 93

Crouania . . . 190, 216

Dictyota . . . . 93

Elachista. . . . 87

Gelidium . . . . 135

Mesogloia. . . . 190

Solenia . . . . 98

Spyridia . . . . 190

Striaria . . . . 93

bispora Crouania . 190

crinita Striaria . . . 93

aureolus Capsosiphon . . . 214

australe Ceramium. . . . . 191

" Lithothamnion . . . 212

"Rivularia . . . . 46

autumnale Phormidium . . 37, 222

aversa Vaucheria . . . . . 74

axillaris spiralis Fucus. . . . 118

bacciferum Sargassum . . . . 122

baculum Lyngbya . . . . . 39

Balani . . ... . . . 48 
Balbisiana Bryopsis. . . . 63,74 Lamourouxii Bryopsis $\mathbf{7 4}$

Balliana Cladophora . . . 68, 216 balticus Ascocyclus. . . . . 111 Desmotrichum. . . . 95 Fucus . . . . 117, 118 Bangia . . . . 94, 127, 213 bangiiformis Porphyra . . . . 1:7 barbatum Callithamnion . . . 177 Ceramium . . . 178 Cystoseira . . . . 121 Griffithsia . . . 178, 219 Spermothamnion . . 177 Battersii Ectocarpus . . 108, 217 bellula Polysiphonia . . . . 169 Berkeleyi Cylindrocarpus . . . 87 Bertolonii Cladophora . . . . 68 Mesogloia . . . . 130

Biasolettianum Ceramium. . . 191 " Polysiphonia. . 171

" Rivularia . • 46 " ciliata Rhodymenia . . 146

" Sphærococcus . . . . 141

biformis Fucus . . . . . . 118

Bifurearia . . . . . . . . 119

Binderianum Plocamium . . . 154

bipinnatum Callithamnion. . . 182 Sphacelaria . . 98, 226 " Stypocaulon . . 98

biscayensis Dermocarpa . . . 32 bispora Crouania. . . . . . 190 Bolbocoleon . . . . . 63 bombycina Conferva . . . . 53 Bonnemaisonia . . . 160, 214 Bonnemaisoniæ Colaconema . . 129 Bonnemaisonii Aglaophyllum. 156 " Delesseria. . . 156 " Nitophyllum . 156 $" \quad$ Oscillatoria - 35.36

Bornetia . . . . . . 179

Bornetii Lithothamnion . . . 212

Borreri Callithamnion . . 180, 183 " Pleonosporium. . . . 180

" fasciculatum Pleonosporium . . . . . 183 Bostrychia. . . . . 161, 214 botryocarpum Ceramium . . . 194 Boucheri Ceramium. . . . 174
Boucheri mucilaginosum Ceramium . . . . . 174

tenuissimum Ceramium $\quad 174$

Bouteillei Hassalia . . . . . 49

brachiatum Callithamnion. . . 186

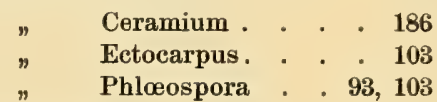

brachygonia Rhodomela . . 161

Brachytrichia. . . . . . . 48

Brebneri Rhodochorton. . . . 180

breviarticulata Elachista . . . 89

brevifructus Ectocarpus . . . 103

brevipes Haligenia . . . . . 83

brevis Oscillatoria . . . . . 35

"Streblonema. . . . . 109

Brodiæi Callithamnion . . . 182, 184

" Ceramium . . . . . 182

" Phyllophora . . . . 140

" Polysiphonia - 165, 168, 223

" simplex Phyllophora. . 141

Brongniartella . . . 173, 214

Bryopsis . . . . . 63, 73, 74

Buffhamii Gonimophyllum . 158

Buffhamia. . . . . . . 86

Buffhamiana . . . . . . . 113

bulbosa. . . . . . . . . 81

bullosus Asperococcus. . . 96

Encœlium . . . . . 96

Enteromorpha. . . . 58

Laminaria . . . . 81

Plysactis . . . . . 47

Rivularia . . . 47, 225

Ulva . . . . . 62

bursa . . . . . . . 76, 216

bursata marina Vaucheria. . . 74

byssoides Brongniartella . . 173, 214

n Callithamnion . . 183, 187

n Ceramium . . . 183

" Hutchinsia . . . 173

" Phlebothamnion . . 187

" Polysiphonia . . . 173

" Seirospora . . . . 187

n seirosporiferum Calli-

thamnion . . . . 188

Cabreræ Carpomitra . . . 83, 214

cærulescens . . . . . .163, 215

cæspitosum Callithamnion . . 131

Castagnea . . 85

Chantransia . . 131 
cæspitosa Conferva . . . . 54

Dasya . . . . . 173

Enteromorpha . . : $\mathbf{5 7}$

Hyella . . . . 33, 219

Laminaria . . . 97

Laurencia . . . 162, 220

Mastichonema . . 45

Mesogloia . . . . 85

Phyllitis . . . . 97

Polysiphonia . . . 165

Punctaria . . 95, 224

cæspitula Sphacelaria . . . 98, 100

calcareum. . . . 206, 212, 220

californicum Stenogramma . . 141

Calliblepharis. . . . 149, 214

callithamnioides Hapalidium. 211, 212

Melobesia 208, 211,

212

Callithamnion $126,130,131,132,173$,

$176,177,178,180,181,182,188$,

189, 190, 193, 214, 225

Callocolax .

144, 214

Callophyllis

144,146

Callymenia

144,199

Calosiphonia

199

Calothrix

43

Calvadosii Corallina

209

Dumontia . . . . 131

canaliculata . . . . . . . 119

cancellatus Hydroclathrus . 98

cannabina Conferva . . . . 54

capillaceum Cystoclonium. . . 145

" Gelidium . . 134, 135

" Pterocladia . 134, 224

capillaris Gloiosiphonia . . 195

" Rhizoclonium . . . 66

Capsosiphon . . . . . . 214

capucina Oscillatoria . . . 36

Carmichælia . . . . . . 93

Carmichæliana Polysiphonia . 170

Carmichælii Sphærozyga . . . 49

carnosum Nitophyllum.

Carpomitra . . . . 83, 214

carpophyllum Hypoglossum • . 160

cartilagineum Gelidium . . 135

Laurencia . . . 168

Sphærococeus. . 135

Casparyi Rhizoclonium . . . 66

Castagnea. . . . 84, 85, 87, 88

Castagnei Myriocladia . . . . 85 cataractarum Chlorotylium . . 63

catenata . . . . . . . 68

Catenella . . . . . . . 146

Catenellæ Symploca . . . . 41

cateniforme Hormoceras . . . 192

Cattlowiæ Dasya . . . . . 173

Caulacanthus. . . . . . 137

Cresswellii Calothrix . . . . 43

celticus Chondrus . . . . . 142

ceramicola . . . . . . 126, 218

ceramiiformis Polysiphonia . . 167

Ceramium 160, 171, 172, 173, 174, 177, $178,180,182,183,184,185,187$,

188, 191, 215

ceranoides Fucus . . . . 116, 118

ceratophylloides Dasya. . . . 173

cervicorne Desmotrichum. . . 95

Goniotrichum . . . 126

Chætomorpha . . . . . 65

Chætophora . . . . . 87, 143

Chætopteris . . . . 100, 215

chalybea Leibleinia. . . . . 44

" Oscillatoria . . . 35, 222

" luticola Oscillatoria. . 36

Chamæsiphon. . . . . . 34

Champia . . . . 154, 215

Chantransia . . 130, 131, 215

Chauviniana Cystoseira . . . 122

Chiajeana Chrysymenia . . . 155

Chilionema . . . . 88, 215

Chlorella . . . . . 52

Chlorochytrium . . . . . 52

Chlorocystis . . . . . . 52

Chlorosiphon. . . . . . . 94

Chlorotylium . . . . . . . 63

Chondracanthus . . . . . . 139

Chondria $146,152,153,154,155,162$,

163,215

chondriformis Fucus . . . 118

Chondriopsis . . . . . . 163, 164

Chondrus . 137, 139, 141, 142, 155

Chorda. . . . . . 82, 94, 96

Chordæ Elachista . . . . . 90

chordalis . . . . . . . 147

Chordarja. . . 83, 84, 85, 130

chordariiformis Castagnea . . 88

Cladosiphon. 85,88

" Myriocladia . 88

Choreocolax . . 133, 134, 215 
Choreonema . . . . . . 203

Chroococcus . . . . . . 31

Chrysymenia. . . . 154, 155

Chthonoblastus . . . . . . 37

chthonoplastes Microcoleus . 42, 221 Chylocladia . . 152, 153, 154, 216

Chylocladiæ . . . . . . . 130

ciliaris . . . . . . . . 127

ciliata Calliblepharis ' . . 149

" Ceramium . . . . 191, 192

, Delesseria . . . . 149

"Dictyota . . . . . . 124

Fucus . . . . . 149

Halymenia . . . . 149

Rhodomenia. . . . 149

"Sphærococeus . . . 149

"Taonia . . . . . . 124

circinatum Ceramium . . . 191, 215

circinans Rhodophyllis . . 146

cirrosum Cystoclonium . . 145, 217

"Sphacelaria . . . . 98

" pygmea Sphacelaria . 99

$"$ simplex Sphacelaria 100

Cladophora . . . . 54, 67, 216

Cladophoræ Chætomorpha. . . 65 Conferva . . 54

Cladosiphon . . . . . 85, 88

Cladostephus. . . . . . 100

clandestinus . . . . . . . 89

clathrata Conferva . . . . 56

"Enteromorpha . 56, 57

Ulva . . . . 56

confervoides Ulva . . $\quad \mathbf{5 7}$

crinitá Enteromorpha . 57

mucosa Enteromorpha. $\quad 57$

uncinata Ulva . . . 59

clavatum Gelidium . . . . 135, 136

Linckia . . . . . 79

Monospora . . . 180

Myrionema . . . . 79

Nemalion . . . . . 131

Ralfsia . . . . . 79

Vaucheria . . . 74

clavellosa. . . . . 154, 216

claviferum Gelidium . . . 135

claviformis Myriotrichia . . 114 filiformis Myriotrichia $\mathbf{1 1 4}$

Cloustonii Laminaria . . . 80

coccineum Ceramium . . . 193

Dasya . . . . 174 coccinea Delesseria. . . . 154

" Dudresnaya . . . 198, 217

" Hapalidium . . . 204

"Heterosiphonia . : 174

" Mesogloia . . . 198

"Nemalion . . . 198

" Plocamium . . . 154

" Porphyra . . . . 128

"Binderianum Ploca-

mium . . . . . 154

" subtile Plocamium . . 155

Codii Acinetospora . . . . 112

" Callithamnion . . . . 131

" Ectocarpus . . . . 107

Codiolum . . . . . . 53, 64

Codium . . . . 75, 216

Coemansii Fucus. . . . . . 118

Cohnii . . . . . . . 52

Colacolepis . . 143, 216, 223

Colaconema . . . . 129, 130

collabens Conferva. . . . . 62

"Hosmiscia. . . : . 62

" Hormotrichum . . 62

" Hutchinsia . . . 165

"Polysiphonia . . 165

" Streblocladia . . . 165

"Ulothrix . . . . 62

collaris. . . . . . . . 78

Colpomenia . . . . . . . 97

colubrina Oscillatoria . . . . 35

Comatula Polysiphonia. . . . 167

comosa Polysiphonia - . . 170, 224

compacta Corallina . . . . 209, 216

" Ectocarpus . . . 102

" Halurus . . . . . 179

complanata Enteromorpha . 56, 57

" Odonthalia . . . 172

" Polysiphonia. . 172

" Pterosiphonia . . 172

" Rytiphloea . . . 172

compressus Asperococcus. . . 96

Enteromorpha . 56, 60

Gigartina . . 138, 148

Gracilaria . . 148

Lithothamnion 206,212, 220

Plocaria . . . 148

Sphrerococcus . 148

Ulva. . . . . 58, 60

lingulata Enteromor. pha . . . . 58 
compressa prolifera Enteromorpha . . . . 59 prolifera Ulva . $\quad 59$

Compsothamnion . . . . 188

concatenata Cystoseira. . . . 121

Gelidium . . . 135

Hypoglossum. . . $\mathbf{1 5 9}$

Phyllophora . . . 140

Conchocelis . . . . . . 73

concinna Leathesia . . . . 86

condensata Grateloupia . . 197

conferta Palmella . . . . 51

Conferva . 53, 56, 62, 65, 66, 67, 68, $69,70,71,74,90,99,194$

confervicola Calothrix . . . . 44

$" \quad$ Hapalidium . . . 212

" Melobesia . . . 212

" Oscillatoria . . . 44

" zostericola Calothrix 44

confervoides Ectocarpus 103, 107, 217

Gigartina * . 148

Gracilaria . . 148, 219

Hapalidium . . 205, 220

Hypnea . . . 148

Lyngbya . . . 39, 40

Plocaria . . . 148

Sphærococcus . . 148

Spyridia . . . 190

arctus Ectocarpus. 107

hiemalis Ectocarpus 107

confine Dermatolithon . . . 205, 217

\begin{tabular}{|c|c|c|c|c|}
\hline & Ectocarpus & . & . & \\
\hline & Melobesia & & . & . \\
\hline & Rivularia & . & . & \\
\hline
\end{tabular}

confluens Rivularia. . . . 46, 47

" Ulvella . . . . . 54

congestus Ectocarpus . . . . 105

" Lyngbya. . . . . 40

" Polysiphonia . . 168

Contarenii Calothrix . . . . 44

Mastichonema . . . 44

" Rivularia . . . . 44

Contarinia. . . . . 200, 201

contorta Castagnea. . . . 85 Fucus . . . . 118

Mesogloia . . . . 85

Tellamia . . . . . 64

convoluta Merismopedia . . 32

Corallina . 77. 204, 209, 212, 216

Corallina Griffithsia . . . 178, 179
Corallinæ Melobesia . . . . 203

Oscillatoria . . . . $\mathbf{3 6}$

coralloides Lithophyllum . . . 206

" Lithothamnion . . 212

" Polysiphonia . . . 175

cordata Schizymenia . . . . 199

Cordylecladia. . . . . 152, 216

coriacea Hypheothrix . . . . 43

Mesogloia . . . . 85

corium Phormidium . . . 37, 38

corneus Chondrus . . . . . 138

Gelidium . . . 135, 218

" Laminaria . . . . 81

attenuatum Gelidium . 135 cæspitosum Gelidium . 136 capillaceum Gelidium . 136

clavatum Gelidium . . 136

crinale Gelidium. - . 136

crinalis Sphærococeus . 136

hypnoides Gelidium . 136

Linnæi Gelidium . . 136

pinnatum Gelidium . . 134

plumula Gelidium . . 136

pristoides Gelidium. 136

" pulchellum Gelidium . 136

" sesquipedale Gelidium. 137

corniculata . . . . . . 210

Cornucopiæ Enteromorpha 57, 58, 60

coronopifolius . . . . . . 147

corticiforme Lithothamnion . . 211

Corunnæ Myrionema . . . . 86

corymbiferum Acrochætium . . 131

" Ceramium . . 194

" Chantransia . 131

" Chylocladia. . . 154

" Dasya . . . 173

" Laurencia . . . 162

" Phlebothamnion . 183

corymbosum . . . . . 183, 214

Corynospora . . . . 176, 177, 180

crassa Chætomorpha . . . . 65

"Heterosiphonia. . . . 174

" Lithophyllum . . . . 206

" Lithothamnion 205, 206, 212, 220

" Melobesia . . . . 206

$"$ Spyridia . . . . . . 191

crassicaulis Ectocarpus . . . 104

crenata Delesseria . . .. . . 159

crepidinum . . . . . . . 31

Cresswellii Schizothrix . . . 43 
crinale Gelidium . . . 136

crinitus Ectocarpus . 104

2. Enteromorpha. . 57, 58

crispata Cladophora . . . . 68

" Dumontia . . . . 198

" Enteromorpha . . . 58

" Fucus . . . . . 117

" Phycoseris . . . . 58

crispula Bangia . . . . . . 127

crispum Antithamnion. . . . 190

Chondrus . . 137, 142, 215

Delesseria . . . . 159

Enteromorpha . . 57, 58

Hypoglossum . . . 159, 220

I.eathesia . . . . 86

Lyngbya . . . . . 40

dubius Sphærococeus . 142

patens Sphærococcus . 142

cristata Callophyllis . . . . 146

Euthora . . . . 146

Lithophyllum . . . . 206

Plocamium . . . . 172

Polysiphonia . . . 172

Rhodomela. . . . 172

Rhodymenia . . . 146

Crouania . . . 190, 196, 216

Crouanii Callithamnion . . . 177

Cladophora . . . . 71

Conferva . . . . 71

Ectocarpus . . 104, 218

Hæmatophlæa . . . 202, 211

Hildenbrandia . . . 211

Lithophyllum . . . 212

Punctaria . . . . 95

cruciatum Antithamnion . . 189, 213

Callithamnion . . 189

" Cruoriopsis . . 201

" Ectocarpus . . . 93, 109

cruenta Petrocelis . . . . 200

Cruoria. . . 200, 201, 216

Cruoriella . . . . . . . 201

cruoriformis Contarinia . . . 201

Cruoriopsis . . . . . . . 201

crustacea Amphithrix . . . . 45

Calothrix . . . . 44

Myrionema . . . . 33

Cryptonemia . . . . . . .197

cryptarthrodia verruculosa Am.

phiroa. . . . . . 210

Cryptopleura . . . . . . . 158 crystallina Cladophora. . . , 68

Ctenosiphonia . . . . . 175

curta Elachista . . . . . 89,90

curvata Strepsithalia . . . . 113

curvula Conferva . . . . . 54

cuspidata Spyridia . . . . . 191

Cutleria . . . . . 78, 217

Cutleriæ Hormotrichum . . . 62

cylindrica Laurencia . . . . 162

Cylindrocarpus . . . . 87, 111

Cylindrospermum . . . . . 49

cymiflora Bornetia . . . . . 179

cystocarpus Fucus . . . . . 117

Cystoclonium . . . . 145, 217

Cystoseira. . . . . . 120

dalmatica Lyngbya . . . . . 39

Dasya . . . 166, 167, 173, 217

Dasyactis . . . . . . . 48

Dasyopsis . . . . . . . 174

dasyphylla Chondria . . . 215, 164

Chondriopsis . . 164

Laurencia . . .163, 164

squarrosa Laurencia. 164

Daviesii secundatum Callithamnion . . . . . 132

Callithamnion . . 131, 132

Chantransia . : . 131, 215

debilis Laminaria . . . . 81, 95

" Phyllitis. . . . . 97

" Punctaria . . . . . 95

decipiens Callithamnion . . . 189

"Colacolepis . . . 143

" Polysiphonia . . . 169

"Sterrocolax . . . 143

decompositum Callithamnion. . 183

decurrens Ceramium . . . 191, 194

Delesseria 140, 141, 144, 147, 149, 154, 156, 157, 158, 160, 195, 217

delicatissima Polysiphonia . . 168

delicatula Porphyra . . . . 128

delicatum Ceramium . . . 194

dendroides Ochlochæte . . . 62

densa Myriotrichia. . . . 114

"Polysiphonia. . . . 165

denticulata Polysiphonia . . . 170

denudata Brongniartella . . . 173

Halidrys . . . . 120

Polysiphonia . . 165, 171

depressum Lithothamnion . . 205

Derbesia . . . . . . 74 
Dermatolithon 204, 205, 212, 217 Dermocarpa . . . . 32, 217 Dermocorynus . . . . . 197 Deschampsii Polysiphonia . . 165 Deslongchampsii Grongroceras 192, 215 Desmarestia . . . . . . . 91 Desmotrichum . . . . 94, 95 deusta Hildenbrandia . . . . 80 " Padina . . . . . . 80 " Ralfsia . . . . . 80 "Zonaria . . . . . 80 devoniensis Griffithsia. . . 178 diaphanum Ceramium . . . 192, 194 " gracile Ceramium . 194 " minus Ceramium - 194 n nanum Ceramium . 193 " rigidum Ceramium . 195 " tenue Ceramium. . 193 " tenuissimum Ceramium .

Dischosporangium . . . . .113

Dichothrix. . . . . 45

dichotoma Agardhia . . . . 76 Conferva . . . 74 Dictyota . . . 123 Grateloupia . . . 197 Halymenia . . . 196 Nemalion . . 130, 222 Nitophyllum . . . 157 Spongodium . . . 76 Vaucheria . . 74, 226 Zonaria . . . . 123 elongata Dictyota. . 124

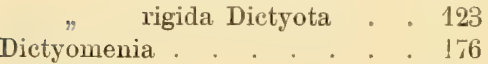

Dictyopteris . . . . . . 125

Dietyosiphon . . . . . 91,93

Dictyota . . 79, 93, 123, 124, 125 dictyotoides Punctaria. . . 95 difforme Codium. . . . 75, 76 " Leathesia . . . . . 86 diffusa Cladophora . . . . 69 digitata Laminaria . . . . 80,81

Dilsea . . . . . . . 198

disciformis Ralfsia . . . . . 79

discigera Erythrotrichia . . . 126

discolor Polysiphonia . . . . 164

discors Cystoseira . . . . . 121

" linearis Cystoseira . . 121 disciplinalis Fucus . . . . . 118 distans Cladophora. . . . 69,72

distichus Fucus . . . . . . 118

" Sphacelaria . . . . 101

"Stypocaulon . . . 102

distortus Eetocarpus . . . 104, 218

divaricata Castagnea . . . . 85

Ceramium . . . . 192

Chordaria . . . 85

Dictyota . . . . 124

Dudresnaya . . . 131

Fucus . . . . 118

Gracilaria . . . 148

Helminthora . . . 131

Mesogloia . . 85, 131

Nemalion . . . 131

Polysiphonia . . 172

Pylaiella. . . . . 102

Spyridia. . . . . 191

divisa Wildemania . . . . . 129

draparnaldioides Ectocarpus . 104, 218

Dubyi Callymenia . . . . . 199

, Euhymenia . . . . . 199

"Halymenia . . . . 199

" Peyssonnelia . . . 202

"Schizymenia. . . . 199

Dudresnaya . . 131, 198, 217

Dudresnayi Callithamnion . 182, 183

Desmarestia . . . 92

Dumontia . . . . 131, 155, 198

Dunalii Monas . . . . . 52

dura. . . . . . . . 148

Durvillaei Gigartina . . . 148

Dyeri Sykidion . . . . . . 53

echinatus Asperococcus ? . . 96

echionotum Ceramium. . . . 192

Ecklonia . . . . . . 82

Ectocarpi Phormidium . . . 38

Ectocarpus 86, 89, 91, 93, 102, 103, $109,110,111,112,113,114,217$

edulis Sarcophyllis. . . . . 198

efflorescens Acrochaetium. . . 130

Ekmanii Dictyosiphon . . . . 91

Elachista . . 86, 87, 89, 91, 112

elegans Bangia . . . . .127, 213

Callithamnion . . . 189

Ceramium . . . . 192

Corallina . . . . 212

Ectocarpus . . . . 107

Goniotrichum . . . 127

Plumaria . . . . 188, 189 
elegans Ptilota . . . . . . 188

. Rhododermis . . . 202

Eleutherospora . . . 205, 211

Elisiæ Rhodymenia. . . . . 151

ellipsosporum Nostoc . . . . 49

Ellisiæ Delesseria . . . . . 141

elongatus Chondrus . . . . 138

Codium . . . . 76

Corallina. . . . . 209

Dictyopteris. . . . 125

Grateloupia . . . 197

Polysiphonia . . . 165

Sphærococcus . . 138

elongella Polysiphonia. . . 166, 223

Encoelium . . . . . . . 96, 97

Endoderma . . . . . 63

Endodictyon . . . . 109, 218

endophlæa Schmitziella . . . 203

endozoicum . . . . . . 130, 213

Enteromorpha . . . 55, 56,61

Ulva . . . 60 compressa Ulva . 56 intestinalis Ulva . 57 lanceolata Ulva . 58

Entophysalis . . . . . . . 33

entophyticum Acrochætium . 130, 213 Nostoc . . . . 49

entozoicum Giardii Rhodochorton 181 Epicladia

64,218

Epilithon . . . . . . . 204, 207

episcopalis Rhodomela . . 163

equisetifolius . . . . . . . 179 simplicifilum Halurus 179 erecta Cordylecladia . . . 152, 216

„Enteromorpha . . 56, 57

Gigartina . . . . 152

Gracilaria . . . . . 152

Sphærococcus . . . 152

ericoides Cystoseira . . . . 121

erythræus Cladosiphon . . . 88

Nemacystus. . . 88

Erythropeltis . . . . . 126

Erythrodermis . . . . . . 211

Erythrotrichia . . . 126, 218

esculenta Alaria. . . . . . 82

Eudesme . . . . . . . 87

Euhymenia . . . . . . 199

europæum Stenogramma . . . 141

Euthora . . . . . . . 146

evesiculosus Fucus. . . . 117 exasperata Ecklonia . . . . 82

expansa Cladophora . . . 68, 216

" Lithophyllum . . . 205

extensa Ralfsia . . . . . . 80

falcata Cladophora . . . . . 72

" Enteromorpha . . . . 57

" Gigartina . . . . 138

" Sphærococeus . . . 138

fallax Callithamnion . . . . 183

farinosa Melobesia . . 203, 212, 221

Fascia . . . . . . . 97

" cæspitosa Phyllitis . . 97

fasciata Ulva. . . . . . . 61

fasciculatum Callithamnion . . 183

Calothrix . . . 44.

Ceramium . . 194

Ectocarpus 104, 105, 218

Leptonema . . . 90

Lithophyllum . . 205

Lithothamnion . 205

Melobesia . . 205

Schizosiphon . 44, 45

Streblonema . . 109

fasciola Dictyota. . . . . . 124

" ligulata Dictyota . . . 124

Fastigiaria. . . ... . . 199

fastigiatum Ceramium . . . 193

" Furcellaria . . . 199

" Gongroceras . . . 193

" Polysiphonia . . 166

fatiscens Lithoderma . . . . 80

Fauchea . . . . . . . 155

favosum . . . . . . 38

Felixii . . . . . . . 183

fenestratus Ectocarpus . . 104, 112

" Giffordia . . . 112

Ferrari . . . . . . 145

ferruginea Lyngbya. . . . 39, 40

ferulacea Polysiphonia. . . . 166

fibrata Polysiphonia . . . . 166

fibrillosa Polysiphonia. . . 166, 170

fibrosa Chætomorpha . . . . 65

$"$ Cystoseira . . . . . 121

filamentosum Ceramium . . 190

filicina "Grateloupia $\quad$. . 190, 191

Halopteris . . . 101, 219

" Sphacelaria. . . . 101

" patens Sphaceleria . 101

filiformis Chondrus. . . . 138 
filiforme Codium . . . . . 76

" Dumontia. . . . . 198

" Enteromorpha . . . 58

" Hypoglossum . . . 159

" Myriotrichia . . . 114

" Phyllitis . . . . . 97

" Pogotrichum . . . . 96

" intestiniformis Dumon-

tia. . . . . 198

filum Chorda. . . . . . . 82

"lomentaria Chorda . . . 96

"trichodes Chorda . . . 94

Finisterræ Calosiphonia . . . 199

firmior Rhodomela . . . . . 162

firmus Ectocarpus . . . .102, 103

" Pylaiella . . . . . . 103

" vernalis Ectocarpus . . 103

fissurata Hæmatocelis . . . . 202

fistulosus . . . . . . . . . 96

flabellata Callophyllis . . . . 144

"Fucus . . . . . 118

" Lithophyllum . . . 205

flabelligerum Ceramium . . . 193

" Lithothamnion . . 220

flaccidum Ceramium . . . . 193

$" \quad$ Elachista . . . . . 89

" Seirospora . . . 187, 225

flaccum Hormidium. . . . . 64

Ulothrix. . . . . 62,64

flagelliformis Chordaria . . . 84

" Enteromorpha . . 58

flavescens Cladophora. . . . 68

flavicans Rhizoclonium . . . 66

flavifolium Sargassum . . . . 122

flavo-fusca Oscillatoria . . . 42

flavum Stypopodium . . . . 79

„Zonaria . . . . . . 79

flexicaulis Laminaria . . . 81

flexuosum Callithamnion . . . 180

" Cladophora. . 54,68, 69

" Enteromorpha . . 57

"Pleonosporium. . . 180

floceosum . . . . . . . . 190

floridulum . . . . . . . . 180

Flustræ Epicladia . . . . 64, 218

Erythropeltis . . . . 126

fœniculacea Cystoseira . . . 121

" Dictyosiphon. . . 91

" Polysiphonia. . . 168

" Scytosiphon . . . 91 foeniculaceus subarticulatus Dictyosiphon . . . . . . 93 foetidissima Polysiphonia . . 166, 223 forcipata Polysiphonia. . : . 167 formosa Polysiphonia . . . 170, 171 fracta Cladophora . . . . . 68 " marina Conferva . . . 71 fragilis Anabæna . . . . . 38 " Phormidium. . . . . 38

" Striaria . . . . 84, 93 fragilissimum Callithamnion . . 189 friabilis Spyridia . . . . . 190 fruticulosum Callithamnion . . 184

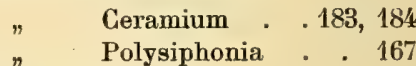
fucescens Physactis . . . . 47 Vaucheria . . : 75 fucicola Elachista . . . . . 89 Fucodium . . . . . . .118, 119 fucoides Polysiphonia . . . 168, 169 Fucus . . . . . . 115, 149 fuliginosa Pleurocapsa. . . . 33 fulva Sphacelaria . . . . . 100 fulvescens Ectocarpus . . . . 102 " Pylaiella . . . . 102

Furcellaria . . . . . . 199; 200

Furcellariæ Callithamnion . . 187 Seirospora. . . 187

furcellata Fastigiaria . . . . 199

" Ginnania . . . . 133

" Halymenia . . . . 133

" Polysiphonia . . 167, 223

" Scinaia . . . . 133

furcigera Sphacelaria . . . 99

fusco-purpurea Bangia . . 127, 128

fuseo-violacea Calothrix . . . 44

fusca Lyngbya . . . . . . 221

"Monostroma . . . . . 55

" Sphacelaria . . . . 98, 99

"Ulva . . . . . . . 55

Gaillonii . . . . . . . 187

gallicum Maschalosporium . . 184

Gastroclonium . . . . 152, 155

gelatinosa Glœocapsa . . . . 31

Gelidiopsis . . . . . 150

Gelidium 134, 135, 147, 150, 163, 218

geniculata Gracilaria . . . 148

genuina Cladophora . . . . 68

Codium . . . . . . 76

Enteromorpha. . . . 56 
genuinum Gelidium. . . . . 136

n Phyllitis. . . . . 97

" Ulva . . . . . . 61

Georgii . . . . . . . 202

Giffordia . . . . 112, 218

gigantea Enteromorpha . . . 58

" Lithophyllum. . . . 205

Phycoseris. . . . . 61

Gigartina $137,138,141,142,145,146$, 148, 152, 164, 195

gigartinus Sphærococcus . . . 139

Ginnania . . . . . . . 133

Giraudia . . . . . . 91

Giraudii Griffithsia . . . . . 179

glabellum Ceramium . . . . 192

glandulosa Delesseria . . . . 195

Microcladia. . . 195, 221

glauca Merismopedia . . . . 32

Protococcus . . . . . 52

glaucescens Cladophora . . . 69

globifer Ectocarpus. . . . . 105

globulosa Elachista. . . . . 90

Gloocapsa . . . . . . 31

Gloeoystis . . . . . . 51

Glœotila * . . . . . . 62

Glœotrichia . . . . . 48

Gloiosiphonia . . . . . 195

glomerata Cladophora . . 68, 72, 216

Ectocarpus . . . 105

Hypoglossum . . 159, 219

Polysiphonia : . . 167

Stypocaulon . . . 102

flavescens Cladophora 71

glutinosum Hydrocoleum . . . 42

Gmelini . . . . . . 156

Gomontia . . . . . 64, 219

Gomontiana Radaisia . . . . 33

Gongroceras . . . . . . 192, 193

Gonimophyllum . . . . . 158

Goniotrichum . . . . 126, 127

Gracilaria . . . 145, 147, 152, 219

gracilior Liebmannia . . . . 88

gracilis Chætomorpha . . . . 65

Cladophora . . . . . 69

Cruoriopsis . . . . 201

Himanthalia . . . . 120

Liebmannia. . . . 88

Lyngbya. . . . . 36

Mesogloia . . . . 87

Physactis . . . . 48 gracilis Plagiospora. . . . . 201

"Rhodomela. . . . . 161

" Rivularia . . . . . 47

gracillimum Callithamnion. . . 188

Ceramium . . . 193

" Cladophora . . . 67

" Compsothamnion. . 188

" Gelidium . . . 135

Grammita . . . . . . . . 171

granifera Corallina . . . . . 209

granosa Glœocapsa. . . . . 31

granulatum Callithamnion . . 184

" Cystoseira. . . 121, 122

granulosus Eetocarpus. . . . 105

" Entophysalis . . . 33

Grateloupia . . . . . . 197

graveolens Lyngbya . . . . 41

gregarium Codiolum ... . . 53

Grevillei Elachista . . . . . 90

Enteromorpha . . . 55

Monostroma . . . 55

Ulva . . . . . 55

Griffithsia 174, 176, 178, 179, 180, 219

Griffithsiæ Chondrus . . . 141

" Gigartina . . . . 141

" Gymnogongrus. . . 141

" Lyngbya. . . . . 40

, Sphærococeus . . . 141

Griffithsiana Gastagnea . . . 85

" Ectocarpus . . . 93

" Haloglossum. . . 96

$" \quad$ Helminthocladia . 85

" Mesogloia . . . 85

" Myriocladia . . . 85

" Polysiphonia . 171, 172

" Seirospora . . 188, 225

" Spyridia . . . 191

" Stictyosiphon . 93, 103

grisea Microchæte . . . . . 48

Guernisaci Polysiphonia . . . 167

Dasya . . . . 167

guttatum Callithamnion . . 182

Gymnogongrus . . . 141, 219

gypsophila. . . . . . . . 45

Hæmatocelis . . . . . 201, 202

Hæmatococcus ... . . . 52

Hæmatophlæa . . . . . 202, 211

Halarachnion . . . . . . . 196

Halidrys . . . . . 118, 120 
Haligenia . . . . . . 81, 83

Haligeniæ Cladophora . . . 69

Halimeda . . . . . . . $7 \boldsymbol{7}$

Haliseris . . . . . . 125

Haloglossum . . . . . . . 96

Halopithys . . . . 163, 219

Halopteris. . . . . 101, 219

Halorhiza . . . . . 84, 93

Halosphæra . . . . . 53, 219

Halothamnion . . . . . 180

Halothrix . . . . . . 91

Halurus . . . . . . 179

Halymenia $133,140,141,145,146,149$, 150, 151, 157, 158, 196, 198, 199

hamifera Bonnemaisonia . . 161

Hapalidii Callithamnion . . . 184

hapalidioides . . . 205, 212, 217

Hapalidium . . 204, 211, 212, 220

Harveyanum Callithamnion . . 186 Nodularia . . . 50 Peyssonnelia . . 202 Spermosira . . 50

Harveyella . . . . . . . 134

Harveyi Lithophyllum . . . . 205 Symploca . . . . 41

Hassallia. . . . . 49

Hauckii Gruoriopsis. . . . . 201

havanensis insidiosa Polysiphonia.

Hecatonema

Helminthocladia. . . 85, 131, 219

helminthoides Gastroclonium . 152

Helminthora . . . . . 130, 131

hemisphærica Rivularia . . . 47

Heredia Phyllophora . . . . 140

hermaphrodita Polysiphonia . 167

Herponema . . . . . 86, 113

Herposiphonia . . . . . 175

Herpothamnion . . . . . . 185

heterocarpa Cryptopleura. . . 158

Halymenia . . 158

heteronema Conferva . . . . 54

heterochloa Conferva . . . . 69

Heterosiphonia . . . . .174

hiemalis Ectocarpus . . . . 107

"Halopteris. . . . 101

" Stypocaulon . . . . 102

Hildenbrandia . . . . . 80, 211

Hildenbrandioides Hapalidium . 204

Hilliæ . . . . . . . . 156, 222
Hilliana Iridæa . . . . , : 145

Himanthalia . . . . . . 119

Hincksiæ Ectocarpus . . . , 105

hippuroides Scytosiphon . . . 84

hirsuta Heterosiphonia . . . 174

Spyridia. . . . . . 191

hirta Cladophora . . . . 69, 216

hispanicus Ascocyclus. . . . 111

Holmesii Ectocarpus . . . . 105

Hookeri Callithamnion. . . 184

fruticulosum Callitham-

nion . . . . 184

Hopkirkii Enteromorpha . . . 59

Hoppii Cystoseira . . . . . 121

Hormactis. . . . . . . 48

Hormidium . . . . . . 62, 64

Hormiscia . . . . . . . 62

Hormoceras . . . . . 192, 195

Hormotrichum . . . . . 62, 64

hospita Amphithrix. ... .. . 48

" Dasyactis . . . . . 48

" Euactis . . . . . 47

" Rivularia . . . . 47

"Zonotrichia. . . . 47

Hudsonii Helminthocladia . . 131

" Mesogloia. . . . 131

humile Callithamnion .. . . . 184

" Cystoseira . . . . 122

" Polysiphonia . . . . 167

Hutchinsia 164, 165, 166, 168, 170, 171,

$172,173,175$

Hutchinsiæ Cladophora . . . 69

Dasya . . . . 173

hyalina Palmella . . . . . 54

hybrida . . . . . . . . 162

hydnoides Calothrix . . . 41, 45

"Sehizosiphon .. 41, 45

" Symploca . . . . 41

Hydroclathrus . . . . . 97, 98

Hydrococcus . . . . . . . 31

Hydrocoleum . . . . . 42, 219

Hydrolapathum . . . . . . 158

Hyella . . . . . . 33, 219

hyperborea Laminaria . . . . 80

Hypheothrix . . . . . . . 43

Hypnea 134, 137, 145, 148, 150, 219

hypnoides Atractophora . . 134

Bryopsis. . . . 73

Ctenosiphonia. . . 175

Naccaria. . . . . 134 
hypnoides Polysiphonia . . 175 Ptilota . . . . 189 Sphacelaria. . . . 101 Hypoglossum. . . 159, 160, 219 Delesseria . . 159 Hystrix Ochlochæte. . . . . 63 "Sphacelaria. . . . 99, 226 Ilea. . . . . . . . . . 97 immarginata Callophyllis . . . 144 implexa Chætomorpha . . . 66 Ghondria . . . . 154 Conferva . . . . . 66 Dictyota . . . . 123 Hormidium . . . . 62 Hormiscia . . . . 62 Rhizoclonium. . . . 66 Ulothrix . . . . . 62 implicatum Codium. . . . . 76 inæquilatera Melobesia . . 204, 212 incrustans Colacolepis . . . 143, 216 Lithophyllum . . . 205 Lithothamnion . . 205 incurvatus Chondrus . . . . 138 infectoria Oscillatoria . . . . 35 infestians . . . . . . 109, 218 inflatus Fucus . . . . . . 118 insidiosa Oscillatoria . . . . 36 , Polysiphonia . . . 167 insignis Ectocarpus . . . . 105, 218

" Oscillatoria. . . . . 36

"Polysiphonia . . . 167

integer Fucus . . . . . . 117

integerrimus Fucus. . . . . 117

intertexta Lomentaria . . . . 154

intermedia Elachista . . . . 90

" Grateloupia. . . 197

Oscillatoria. . . . 35

interruptum Callithamnion . . 188

" Delesseria . . . 141

" Seirospora . . . 188

n Stenogramma . 141, 226

intestinalis Enteromorpha - 57,61

"Ulva. . . . . 61

" capillaris Ulva . . 60

" Cornucopiæ Entero. morpha . . . maxima Enteromorpha . . . 58,61 micrococca Ulva. . 59 intestinalis prolifera Enteromor-

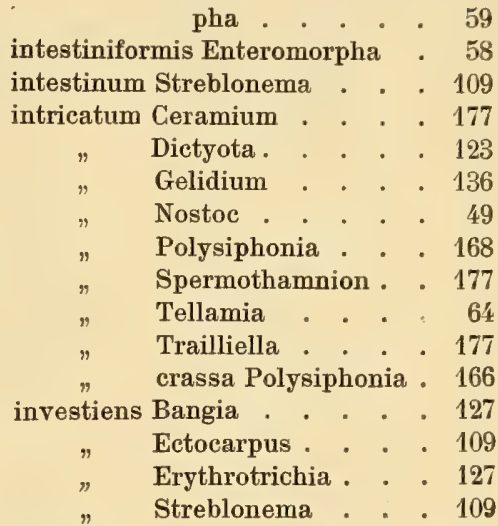

Iridæa . . . . . . . . 145, 198

irregularis Chantransia . . . 132

” Ectocarpus . . . 108

Isactis . . . . . . 46, 220

isogona Ulothrix. . . . . . 64

Isthmoplea . . . . . . 109

Janczewskia . . . . . . . 175

Jania . . . . . . 209, 210

jubata . . . . . . . . 149, 214

Juliana Lyngbya. . . . . . 39

juncicola Schizosiphon. . . . 45

Jurgensii . . . . . . . . 57

kaliformis Chondria . . . . 152

" Chylocladia. . . 152

" Gastroclonium . . 152

" Gigartina. . . . . 152

" Lomentaria . . . . 153

Kochianum Rhizoclonium . . . 66

Kuetzingianum Phormidium . . 42

Kuetzingii Chætomorpha . . . 65

Kurzii . . . . . . . . . 43

labyrinthiformis Spirulina. . . 34

lacerata Delesseria . . . . 157

Halymenia . . . . 157

Nitophyllum . . . . 157

Monostroma . . . . 55

uncinatum Nitophyllum. 158

uncinata Delesseria . . 158

lacerus Chondrus . . . . . 138

laciniata Callophyllis . . . . 144

Delesseria. . . . 144

" Porphyra . . . . . 128

" Rhodymenia . . . 144, 151 
laciniatus Sphærococcus . . . 144

Ulva . . . . 61

"Wildemania. . . 128, 227

" flabellata Callophyllis . 144

" umbilicalis Porphyra . 128

lacinulata Ulva . . . . . . 62

Lactuca Cryptonemia . . . . 197 Ulva. . . . 55, 61, 62 latissima Ulva . . . 61

" rigida Ulva . . . 61,62

" seminervis Cryptonemia 197

lacustris Hæmatococcus . . . 52

"Protococcus . . . . 52

lætevirens Cladophora. . . 70, 72

" Oscillatoria. . . . 36

lætus Ectocarpus . . . . 105, 107

lævigata Polysiphonia . . . . 167

Laminaria . . . 80, 95, 97, 220

Laminariæ Asperococcus . . . 94

Bangia . . . . . 94

Chlorosiphon . . . 94

Dermatolithon. . . 205

Desmotrichum . . 94, 95

Lithosiphon. . . 94, 95

" Melobesia . . . 205

" Punctaria . . . . 94

laminarioides Punctaria . . . 96

Lamourouxii Callithamnion . . 185

Ceramium . . . 185

Derbesia . . . 74

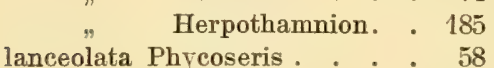

Phyllitis. . . . . 83

Ulva . . . . 60,61

" ramifera Phycoseris . 59

Landsburgii Ectocarpus . . . 106

lanosum Callithamnion . . . 184

Cladophora . . . 70

Conferva . . . . 70

Ectocarpus . . . 106

Mesogloia . . . . 85

lapathifolia Phycoseris. . . 62

$$
\text { " Ulva . . . . 58,62 }
$$

lardacea Schizothrix . . . 43

lasiopus Schizosiphon . . . . 44

laterifructus Fucus . . . . 117, 118

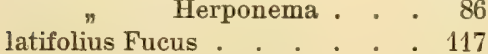

Gelidium . . . 136, 218

Halymenia. . . . 196 latifolia Laminaria. . . . . 81

"Nitophyllum . . . . 157

" Punctaria . . . . 95

" Rhodymenia . . . . 152

" Zosteræ Punctaria - 95, 96

latifrons Chondrus . . . . 138, 215

" Dictyota . . . . . 123

" Fucus . . . . . . 115

" Plocamium. . . . . 155

latissimum Monostroma . . . 55

"Ulva. . . . . . 61

" umbilicalis Ulva . 61, 62

Laurencia . . . . 162, 164, 220

laxa Rivularia . . . . . . 47

"Spirulina . . . . . . 35

Leathesia . . . . . . 86, 87

Lebelii Ectocarpus . . . . 112

" Elachista. . . . . 112

" Giffordia . . . . .112, 218

"Sphacelaria . . . . 98

Leclancheri Myrionema . . 87

Leibleiniæ Dermocarpa. . . 32, 217

" Sphøenosiphon. . . 32

Le Jolisii Aphanothece. . . . 32

" Bangia . . . 128, 213

" Melobesia. . . .204, 221

" Spermatochnus. . . 83

" Stilophora . . . . 83

Lenormandiana Rivularia. . . 46

Balani Euactis . 46

Lenormandii Callithamnion . . 132

" Lithophyllum . . 207

" Lithothamnion . . 207

" Melobesia. . . 207

". Rhizoclonium . . 67

" Schizosiphon. . . 45

Lens Phyllactidium . . . . . 54

"Ulvella. . . . . . 54

lepadicola Rhodochorton . . 181

leptochæte Endoderma. . . . 63

Leptonema . . . . . . 90

leucosticta Porphyra . . . 128, 227

Leveillei Liebmannia . . . 88

Liagoræ Strepsithalia . . . . 113

Liagora . . . . . 131, 133

lichenoides . . . . . . . 207

Liebmannia . . . . . . 88

ligulata Desmarestia . . . . 92

"Dictyota. . . . . . 124

" Halarachnion . . . 196 
ligulata Halymenia . • • . 196 Rhodymenia . . . 150

Sphærococeus . . . 150

Sporochnus . . . 92

igustica Griffithsia . . . . . 179

limitaneus Fucus. . . . . . 116

limosa chalybea Oscillatoria . . 36 subfusca Oscillatoria . . 38

linearis Calliblepharis . . . . 149

" Dictyota. . . . . . 124

$"$ Fucus . . . . . . 118

" Porphyra . . . . 128, 129

"Wildemania. . . . 129, 227

lineolata Zonaria. . . . . . 93

lingulata Delesseria. . . . . 159

" Enteromorpha . . . 58

" Hypoglossum. . . . 159

linifolium Sargassum . . . . 122

Linkia . . . . . . . 79, 88

" Nostoc. . . . . . . 49

" Rivularia. . . . . . 49

Linkiana Enteromorpha . . . 56

Linum Chætomorpha . . . . 65

Linza Enteromorpha . . 58,60

" Phycoseris. . . . . 58, 62

Lithocystis. . . . . 211, 220

Lithoderma . . . . . . 80

Lithophyllum. . . 205. 207, 212

Iithothamnion $\quad 204,205,206,211$,

212, 220

Lithosiphon . . . . . . 94, 95

litoralis Ectocarpus . . . . 102, 103

" Oscillatoria. . . . 42

Palmella. . . . . 52

Pylaiella. . . . . 102

Ectocarpus . . . . 106

compacta Pylaiella . . 102

varia Pylaiella. . . . 106

litorea Lyngbya . . . . . . 38

"Nodularia. . . . . . 50

" Rhizoclonium . . . . 67

"Spermosira . . . . 50

"Vaucheria . . . . 74, 226

$"$ crassior Spermosira . . 50

livida Lyngbya . . . . . . 39

"Porphyra . . . . ., . 128

Lloydiana Oscillatoria . . . . 36

Lloydii . . . . . . . . 48

lobatum Nitophyllum . . . . 157

lobata Phycoseris . . . . . 61 lobata Sarcophyllis . . . 145, 198

Lomation Cryptonemia. . . . 197

Lomentaria . . 146, 152, 153, 154

Chorda. . . . 96

Scytosiphon . . . 96

longifructus Ectocarpus . . . 106

" Fucus . . . . 118

longifurca . . . . . . . . 209

longipes Ectocarpus. . . . . 107

Lophosiphonia 168, 175, 220, 221

Lophothalia . . . . . . 174

lorea Himanthalia . . . . 119

lubrica Gigartina. . . . . 195

Nemalion . . . 130, 222

Prasinocladus . . . 51

lumbricalis Ectocarpus . . . 91

" Elachista . . . . 91

" Furcellaria. . . . 200

" Halothrix . . . . 91

" Polyides . . . . 200

lutarius Fucus . . . . . 115, 118

lutea Laurencia . . . . . . 162

" Lyngbya . . . . . . 39

luteo-fusea Lyngbya $\quad . \quad . \quad . \quad 40$

" " subviridis Lyngbya . 39

luteolum Streblonema . . . 110

lutescens Cladophora : . . . 71

luxurians Callithamnion . . . 131

" Geramium . . . . 193

" Chantransia . . . 133

" Gelidium. . . . . 135

lycopodioides Rhodomela . . 161, 225

Lyngbya . . . . 39, 62, 221

Lyngbyaceum Hydrocoleum . 42, 219

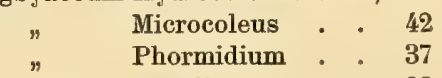

Lyngbyanum Encoelium • • . 96

Lyngbyei Stilophora. • . . . 83

Macallana Cladophora . • . . 70

macrocarpum Dermatolithon 205, 212,

Ectocarpus . . 106

$" \quad$ Ectocarpus . . . 118

" Polysiphonia . 168, 169

macrocystus Fucus . . . . 118

macrodon Fucus. . . . . . 117

maculans . . . . . . . . 88

Magdalenæ Cladophora. . . . 70

magma Protococcus. . . . . 53

major Ectocarpus . . . . 106 
major Liebmannia . . . . . 88

Nodularia. . . . . . 50

Polysiphonia. . . . 170

Pylaiella . . . . . . 103

Spermosira . . . . . 50

Spirulina. . . . . . 34

majuscula Lyngbya . . . . . 40

Oscillatoria . . 36,39

mamillosus Ghondrus . . . . 139

Gigartina . . . 139

Lithothamnion . 206, 220

Mastocarpus . . 139

Rhodymenia . . 139

Sphærococcus. . . 139

margaritifera Oscillatoria . . . 36

marginata Enteromorpha . . . 59

marginiferum Nemastoma. . . 199

Platoma . . . 199

Rhodymenia. . . 151

marinus Chamæsiphon. . . . 34

Cladophora . . . . 69

Derbesia . . . 74, 75

Glœotila . . . . 62

Hydrococcus . . . . 31

Leathesia . . . . 86

Oncobyrsa. . . . . 31

Prasiola . . . . 54

Protococcus . . . . 52

Protoderma . . . . 54

Ulothrix . . . . . 62

Vaucheria . . . 74, 75

maritima Pilinia. . . . . . 63

Vaucheria . . . 75

ellipsospora Anabæna. 49

Martensiana Polysiphonia. . . 167

Maschalosporium . . . . . 184

Mastichonema. . . . . 44, 45

Mastigocoleus. . . . . 48, 221

Mastocarpus . . . . . . . 139

maxima Enteromorpha. . . . 58

mediterranea Corallina. . . 209, 210

Palmella . . 32, 52

melanoidea Aglaozonia. . . . 79

Melagonium Chætomorpha. . . 65

Melobesia 203, 205, 206, 207, 211,

212, 217, 221

membranacea Corallina . . 204

Epilithon . . . 204

Lithothamnion. . 204

Melobesia 204, 217, 221 membranaceum Rhodochorton 181, 225

membranifolia Chætophora . . 143

Phyllophora . 140, 222

Phyllotylus . . 140

mempiscus Fucus . . . . . 118

Meneghiniana Leibleinia . . . 40

$\begin{array}{lll} & \text { Lyngbya. . . . } 40 \\ " & \text { Oscillatoria. . . } 34\end{array}$

" Spirulina. . . 34

Meneghinii Sphærococcus. . . 150

Meredithia. . . . . . . 145

Merismopedia. . . . . . 32

Mertensia . . . . . . 183, 187

Mertensii . . . . . . . 114

mesenteriformis Enteromorpha . 58

mesentericum Nostoc . . . 86

Rivularia. . . . 47

mesocarpum . . . . . . . 181

Mesogloia 85, 87, 88, 130, 131, 190,

198

mesoleptum Cylindrospermum . 49

Microchæte . . . . . 48

Microcladia . . . . 195, 221

micrococea Enteromorpha. . . 59

microcoleiformis Oscillatoria. . 42

Microcoleus . . . . 42, 221

Microcoryne . . . . . . 85

Microcystis . . . . . . 51

microspermus Spermatochnus . 83

microphylla Callymenia . . 144, 145

Gastroclonium . . 153

$" \quad$ Halymenia . . . 145

"Meredithia . . 145

micropterum . . . . . . . 178

microscopicum Acrochætium . . 132

$" \quad$ Callithamnion. . 132

" Chantransia . . 132

" Cylindrocarpus . 87

" Lyngbya . . . 39

microspongium Ectocarpus . . 106

microspora Fauchea. . . . . 155

Microsyphar . . . . . . . 94

Millepora . . . . . . . . 206

miniata. . . . . . . 38

minimus Ectocarpus . . 106, 110

Grateloupia . . . 197

Porphyra . . . . . 128

Spermothamnion. . . 177

Streblonema . . . 110

minus Callithamnion. . . 182 
minus Ceramium . . . . 192, 193

Chorda . . . . . . 82

" Halidrys . . . . . 120

"Schizymenia. . . . . 199

minuta Chætomorpha . . . . 65

" Gymnogongrus. • . . 142

Hypoglossum . . . . 159

Lithothamnion. . . 206, 212

Rhodochorton . . . . 181

minutissima Chantransia . . . 133

minutula Elachista . . . . . 90

mirabile Acrochætium . . . . 132

Callithamnion. . . 132

Chantransia . . . 132

Choreocolax . . . . 134

Harveyella. . . . . 134

Trentepohlia . . . 132

Mitchellæ Ectocarpus . . . . 108

Monas . . . . . . . . 52

moniliforme Phormidium . . . 38

monocystus Fucus . . . . . 118

Monospora. . . . . . . . 180

Monostroma . . . . . . 55

Montagnei Cystoseira . . . . 120

Dermocorynus. . . 197

$" \quad$ Polysiphonia . . 171, 172

mucilaginosa Dasya. . . . . 174

Mucor Lyngbya . . . . . . 41

mucosus Schizosiphon . . . . 45

multifidum Callithamnion . . . 176

Chordaria . . . 130

Corynospora . . . 176

Cutleria . . . 78, 79, 217

Griffithsia * . . 176

Helminthora . . 130

Nemalion . . . 130

Mesogloia . . . . 130

Polysiphonia . . . 168

Sphondylothamnion 176, 226

Wrangelia . . . 176

multipartita Gracilaria . . . 149

Sphærococcus . . 149

musciformis Hypnea . . . 150, 219

muscorum Symploca . . . . 41

Myriactis . . . . . . . 87

myriocarpus Ectocarpus . . . 106

"Melobesia. •. . 204

Myriocladia . . . . 85, 87, 88

Myriocladiæ Ectocarpus . . . 110

Streblonema . . 110
Myrionema 79, 86, 88, 90, 109, 111, 113,

222

Myriophyllum Cladostephus . . 101

Myriotrema . . . . . . . 62

Myriotrichia . . . . 113, 114

Naccaria . . . . 134, 222

nana Corallina . . . . 209. 216

" Enteromorpha . . . . 57

” Sphacelaria . . . . . 99

natans Oscillatoria . . . . . 37

Nathaliæ Chilionema . . . 88, 215

neapolitana Oscillatoria . . . 35

Neesiorum Cladophora . . . 70

Neevia . . .

neglectus Callocolax . . . 144, 214

Nemacystus . . . . . . 88

Nemalion . 130, 131, 198, 201, 222

Nemalionis Leibleinia . . . . 43

Nemastoma . . . . . . . 199

nervosa Delesseria . . . . 140

" Phyllophora . . . . 140

" Halymenia . . . . 140

" Sphærococcus. . . . 140

nicæensis Halymenia . . . . 151

" Phyllophora . . . 141

" Rhodymenia. . . 151

nigrescens Hutchinsia . . . . 168

" Microcoleus . . . 43

" Polysiphonia . . 168

nigro-viridis Oscillatoria . . . 36

nitida Cladophora . . . . . 70

n. Hyella . . . . . . 34

" Physactis . . . . . 47

" Rivularia. . . . . . 47

Nitophyllum . . . . 156, 222

nodosum Ascophyllum. . . 118, 213

" Ceramium. . . . 195

"Fucodium. . . . . 118

" Halidrys . . . . 118

" Ozothallia. . . . . 118

Nodularia . . . . . . 50

nodulosum Ceramium . . . . 194

Norstedtii Spirulina . . . . 34

norvegicus Chondrus . . . . 142

" Gymnogongrus. . 142, 219

" Plectonema . . . 41

"Sphærococcus . . 142

Nostoc . . . . . . . 49, 86

Nostocorum Plectonema . . . 41 
obscura Calothrix . . . . . 39

Lophosiphonia . . 175, 220

Lyngbya . . . . . 39

Monostroma . . . . 55

Polysiphonia - 175, 220, 221

obtusa Chondria. . . . . . 162

$"$ Fucus. . . . . . 118

" Laurencia. . . . . . 162

oceanica Oscillatoria . . . . 35

" Palmella . . . . . 52

" Spirulina . . . . . 35

ocellatus Ascocyclus . . . . 113

$"$ Ceramium. . . . 173, 174

" Dasya. . . . . 173, 217

" Delesseria. . . . 157

" Hutchinsia . . . . 173

" Microcoryne . . . 85

" Myrionema . . . . 113

" Nitophyllum . . . 157

" Phycocelis. . . . . 113

Ochlochæte . . . . . . 62

octosporum Callithamnion. . . 185

Odonthalia. . . . . . . . 172

officinalis Corallina. . . . 209, 216

Okenii Oscillatoria . . . . . 36

olivacea Sphacelaria . . . . 99

" radicans Sphacelaria . 100

Oncobyrsa. . . . . . . . 31

opaca Polysiphonia - 169, 220, 221

Ophidocladus. . . . . . . 175

opposita Pylaiella . . . . . 103

Opuntia Catenella. . . . 146

" Chondria . . . . 146

" Gigartina . . . . . 146

" Griffithsia . . . . 179

" Halimeda . . . . 77

"Halymenia . . . . 146

" Lomentaria . . . . 146

orbicularis. . . . . . . 111

orbiculatum Monostroma . . . 55

ornata Schimmelmannia . . . 196

ornithocephala Vaucheria. . . 75

aversa Vaucheria 74

oscillarioides Anabæna . . . . 49

$"$ Spirulina . . . 34

Oscillatoria . . . . . 35, 222

Osmunda Laurencia. . . . . 163

ostendensis Ectocarpus . . . 112

Ostreobium . . . . . 73, 222

ovalis Chondria . . . . . . 153 ovalis Chylocladia. . . ... 153

" Gastroclonium . . . 153

" Hypoglossum . . . . 159

" Lomentaria . . . . 153

" Valonia . . . . . 77

ovatus Ectocarpus . . . . . . 106

oxycoccum. . . . . . . . 55

oxysperma Ulva. . . . . . 55

Ozothallia . . . . . . . . 118

pachycaulon Phlebothamnion. . 187

pachydermus . . . . . . . 134

pachyphyllus Fucus. . . . . 118

Padina . . . . . 78, 80, 124

Padinæ Giffordia. . . . . . . 112

pallens Callithamnion. . . . 181

" Rhodochorton . . . . 181

" Thamnidium . . . . 181

pallida Aphanothece . . . . 32

" Laminaria . . . . 81

" Palmella. . . . . . 32

" Polycystis . . . . 32

palmata Calliblepharis . . . . 149

" Halymenia. . . . 150

" Monostroma . . . . 55

" Rhodymenia . . . 150

" Sphærococcus . . . 150

palmatifidum Lithothamnion . . 212

Palmella . . . . . . 51

palmetta . . . . . . . 151

" crassiuscula Rhodymenia 152

" nieæensis Rhodymenia. 151

palmettoides Phyllophora . . 141, 223

n nicæensis Phyllo-

phora . . . 151

pannosa Calothrix . . . . . 45

" Cystoseira. . . . . 121

" Laurencia . . . . . 162

" Schizosiphon . . . 45

" Gelidiopsis . . . . 150

" Gelidium . . . . 150

papillosum Myrionema. . 86, 222

$" \quad$ Stilophora . . . . 84

papyracea Oscillatoria. . . . 38

" Phormidium . . . 38

paradoxa Chordaria . . . . 83

" Enteromorpha . . . 59

$"$ Spermatochnus . . 83, 225

parasitica Acrochæte . . . . 63

n Anacystis . . . . 32

$"$ Calothrix. . . . . 44 
parasitica Hæmatocelis. . . . 202

Hutchinsia . . . . 172

Polysiphonia. . . . 172

Pterosiphonia . . 172, 224

Rhododermis. . . 203

Rivularia. . . 44, 46

Schizosiphon. . . 46

parietina Calothrix . . . . . 45

Paroliniana Glooeystis . . . 51

parvula Aglaozonia . . . . 78, 79

Ceramium . . . . . 185

Champia . . . .154, 215

Chondria . . . . 154

Chylocladia. . . . 154

Ectocarpus . . . . 106

Lomentaria. . . . 154

patens Chondrus . . . . . 138

Chylocladia. . . . . 154

Cladophora. . . . . 70

Heterosiphonia . . 174

Lomentaria. . . . 152

Polysiphonia . . 170, 171

patentirama Laurencia. . . . 162

patentissima Sphacelaria . . 99

patula Polysiphonia. . . . . 167

Paronia Padina . . . . . . 124

pectinata Gigartina. . . . 139

" Gelidium . . . . 136

" Polysiphonia. . . . 169

pectiniformis Cladophora . . . 71

pedicellatum Callithamnion . 180, 193

Ceramium . . 180, 193

Corynospora . . 180

Monospora . . . 180

clavata Monospora. 180

pedunculatus Sporochnus. . 83, 226

pellita Cruoria . . . 200, 201, 216

pellucidum Ceramium . . . . 194

Cladophora. . . . 71

peltiformis Actinococcus . . . 143

Pelvetia . . . . . . . .119

penicillata Cladophora. . . 68,71

Ectocarpus . . . 104

Griffithsia . . . 174

Hutchinsia . . . 165

Microcoleus. . . . 43

Wrangelia . . . 174

penicilliformis Urospora . . . 64

pennatum Ceramium . . . 172, 193

Hutchinsia . . . 172 pennata Polysiphonia . . . . 172

Pterosiphonia . . 172, 224

" Sphacelaria . . . 98, 99

percursa Enteromorpha . 59,60 marina Oscillatoria . . 42

Perreymondii Polysiphonia . 171, 172

persicinum Phormidium . . . 38

Petrocelis . . . . . . . 200

peucedanoides Polysiphonia . . 172

Peyssonnelia . . . . 78, 201

peyssonneliiformis Contarinia . 200

Phæocelis . . . . . . . . 113

Phæocystis . . . . . 94, 222

Phæosphærium . . . . . . 88

Phæostroma . . . . . 109, 114

phalligera Lomentaria . . . 153

Pharaonis Oscillatoria . . . . 36

Phlebothamnion 182, 183, 184, 185, 187

Phlæospora . . . . 93, 95, 103

Phormidium . . . 36, 37, 222

Lyngbya . . 40, 41

Phycocelis . . . . . 88, 113

Phycophila . . . . . . . 90

Phycoseris. . . . . 61, 62, 58

Phyllactidium. . . . . . . 54

Phyllaria .. . . . . . . 83

Phyllitis . . . . . . 83, 97

" Laminaria. . . . 81

phyllocarpus Fucus. . . . . 118

Phyllophora . . . 140, 222

Phyllotylus . . . . . . . 140

Phymatolithon . . . . . 205, 211

Physactis . . . . . . . . 48

piliferum Bolbocoleon . . . . 63

" Physactis. . . . . 47

"Sphondylothamnion 176, 226

Pilinia . . . . . . . . 63

piloboloides Vaucheria. . . . 75

pilosum Ceramium . . . . . 192

pilularia Bonnemaisonia . . . 160

pinastroides Callithamnion . . 178

" Halopithys . . 163, 219

" Rhodomela . . . 163

" Rytiphloea . . . 163

" episcopalis Lauren.

cia . . . . 163

pinnata Corynospora . . . 180

Griffithsia . . . . 180

Halothamnion . . . 180

Pleonosporium. . . 180 
pinnatifida Chondria . . . 163

Gelidium . . . 163

Laurencia . . . . 163

eylindrica Laurencia . 162

pinnatinervia Desmarestia . . 92

pinnulata Polysiphonia. . . . 172

pistillata Gigartina . . . . . 139

Placoma . . . . . . . 31

Plagiospora . . . . . . . 201

plantaginea Laminaria . . . . 95

Punctaria . . . 95

Ulva. . . . . . 95

Zonaria. . . . . 95

$\begin{array}{rr} & \text { tenuior Zonaria . . } \\ \text { planus Chondrus . . . } & \mathbf{9 5}\end{array}$

" Dasyactis . . . . . 46

$"$ Isactis . . . . . 46, 220

" Rivularia . . . . 46

Platoma . . . . . . . . 199

platycarpus Fucus . . . . 116, 117

Plectonema . . . . . . . 41

Pleonosporium . . . 180, 183

Pleurocapsa . . . . . . . 33

plicata Ahnfeltia. . . . . . 142

" Gigartina. . . . . 142

" Gymnogongrus. . . . 142

" Physactis. . . . . . 47

" Sphærococcus . . . . 142

" Rivularia. . . . . . 47

"Tylocarpus . . . . 142

Plocamium. 147, 154, 160, 161, 172

"Delesseria . . . 154

Plocaria . . . . 147, 148, 155

pluma Callithamnion ... . . 178

" Ptilothamnion . . . 178

" micropterum Callitham-

nion . . . . 178

Plumaria . . . . . . . 188

plumigera Sphacelaria. . . . 99

plumosa Bryopsis . . . . . 73

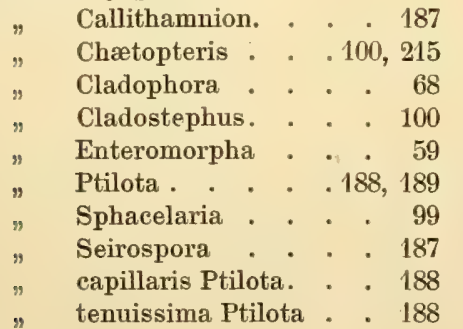

plumula Antithamnion . . . 190

" Callithamnion, . . . 190

" Ceramium . . . . 190

". Pterothamnion . . 190

" Sphacelaria . . . 100

Pogotrichum . . . . . . . 96

polaris Cladophora . . . . . 67

Pollexfenii Callithamnion. . . 190

" Nitophyllum . . 156

polycarpus Fucus . . . . . 118

$" \quad$ Sphærococcus - . 149

polychotoma Polysiphonia, . . 168

polychroa Lyngbya . . . . . 40

polyclados Chondria . . . 153

polycladum Myrionema. . . . 86

Polycystis. . . . . . . . 32

Polyides . . . . . . . 200

" Callymenia . . . . 144

" Rhodomenia . .. . 144

" Sphærococcus. . ... 144

polymorphum Codium . . . . 76

Eleutherospora 205, 211

Lithothamnion 205, 211

Melobesia . . 211

Phymatolithon 205, 211

polyotis Rivularia . . . . . 47

polypodioides . . . . . . 125

polyrhizum Codiolum . . . . 64

Gomontia . . 64, 219

Polysiphonia 164, 173, 175, 220, 223

Polysiphoniæ Choreocolax. . 133, 215

polyspermum Callithamnion . . 214

Ceramium . . 185, 187

Vaucheria . . 75

polyspora Polysiphonia . . . 169

Porphyræ Microsyphar. . . . 94

Porphyra . . . . 127, 128, 129

Pouchetii . . . . . . . 94, 222

prasina Dermocarpa . . . . 32

Prasinocladus . . . . . 51

Prasiola . . . . . . 54

Pringsheimia . . . . . . 54

Pringsheimii Ectocarpus . . . 109

procerrima Enteromorpha. - . 57, 58

Gracilaria . . . . 148

procumbens Corallina . . . . 209

proliferum Ceramium . . . . 194

Cladophora. . . 71

Enteromorpha. . . 59

" flaccida Conferva . . 68 
prolongata Bryopsis. . . . . 73

propinquus Fucus . . . . . 118

Protococcus . . . . . . 52

Protoderma . . . . . . 54

prototypus Hildenbrandia. . . 211

pseudo-digitata Laminaria. . . 81

pseudo-sericea Cladophora . . 69

pseudo-siliculosus Ectocarpus . 107

pseudo-tenuissima Spirulina . . 34

Pteridium . . . . . 160, 224

Pterocladia . . . . .134, 224

Pterosiphonia. . . . 172, 224

Pterothamnion . . . . . 190

Ptilota . . . . . 188, 189

Ptilothamnion . . . . .178

pulchellum Callithamnion . . 180

" Ectocarpus . . . 106

$"$ Gelidium . . . 136

pulcherrimus . . . . . . 200

pulchrum Acrochætium . . . 130

pulvinata Calothrix . . . . 45

Elachista . . . 87

Gelidium . . . 136

Myriactis. . . . . 87

Hutchinsia . . . . 169

Polysiphonia 168, 169, 223, 224 subcostata Ginnania . 133

pumilum Antithamnion . . . 189

Punctaria . . . . 94, 95, 96, 224 punctatum Aglaophyllum . . . 157

Delesseria . . . 157

n Nitophyllum . . 157, 222

" alliaceum Nitophyllum 156

punctiformis Linkia. . . . 88

Myrionema . . 87, 88

" Phæosphærium . . 88

punctulata Glœotrichia . . . 48

punicea Dasya . . . . . . 174

purpurascens Cladophora . . . 71

Cystoclonium . 145, 217

Gigartina . . 145

Gracilaria . . 145

Hypnea . . . 145

Laminaria ... . 83

Phyllaria . . 83

Sphærococcus . 145

crispata Halymenia 198

purpurea Cruoria . . . . 201, 217

" Helminthocladia . 131, 219

n Lithothamnion . . 211 purpurea Mesogloia . . . . 131

Nemalion. . . . 131

Porphyra. . . . . 129

Symploca . . . . 41

Ulva * . . . 129

pusilla Acinetospora . . . 112

Asperococcus . . . . 94

Chlorosiphon . . . . 94

Codiolum . . . . 53

Conferva . . . . 99

Ectocarpus . . . 105, 112

Gelidium . . . . 136

Lithosiphon . . . 94

Lomentaria . . . . 153

Punctaria . . . . 94

Ralfsia . . . . . . 79

Sphacelaria . . . $\quad 99$

Stragularia . . . . 79

pustulata . . . . . 204, 212, 221

pustulosum Phæostroma . . . 114

Pyenophycus . . . . . . . 119

pygmæus Ectocarpus . . . . 108

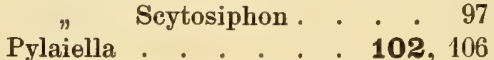

pyramidalis Bryopsis . . . . 74

„ Chylocladia . . 154, 216

"Laurencia . . . 162

pyramidatum Callithamnion . . 184

" Laurencia . . . 162

quaternarium Monostroma . . 55

Quekettii Ostreobium . . . 73, 222

Rabenhorstii Callithamnion . . 187

racemus Lithophyllum. . . . 206

Radaisia . . . . . . . 33

radiata exasperata Ecklonia . . 82

radicans Sphacelaria . . . . 100

Ralfsia . . . . . . . . . 79

Ralfsii Callithamnion . . . 177

" Enteromorpha. . . . 59

ramosa Phycoseris . . . . . 61

ramulosa Enteromorpha . . . 59

rectangularis Cladophora . . . 71

recurvatum Hypoglossum. . . 159

reflexa Bangia. . . . . 127

" Chylocladia . . . . 153

" Erythrotrichia . . . 127

" Gastroclonium. . . 153

n Lomentaria . . . . 153

" Porphyra . . . . . 127

refractum Callithamnion . . 190 
refracta Cladophora * . 54,67, 71

" Ectocarpus . . . 104

reniformis Callymenia. . . . 144

" Halymenia . . . 145

" Iridæa . . . . . 145

" Laminaria . . . 83

" Phyllaria . . . . 83

$" \quad$ Rhodomenia . . . 145

repens Acrochæte. . . . . 63

Gallithamnion . . . 177

Chondrus . . . . 155

Cladophora. . . . . 72

Derbesia . . . . 74

Dichosporangium . . . 113

Ectocarpus . . . . 110

Fauchea . . . . 155

Myriotrichia . . . . 113

Neevia . . . . . 126

Plocaria . . . . . 155

Spermothamnion . . 177, 226

Sphærococeus . . . 155

reptans Aglaozonia . . . . 78, 213

Ascocyclus . . . . 113

Chilionema . . . . 89

Ectocarpus . . . 89, 110, 113

Myrionema . . . . . 113

Nitophyllum . . . . 157

Phycocelis . . . . . 113

Streblonema . . . . 110

Zonaria . . . . . 78

reticulatum Colaconema . . . 129

"Zygomitus. . . . 64

retroflexa . . . . . . 54

Rhizoclonium. . . . . . 65, 66

rhizodes Chordaria . . . . 84

$"$ Spermatochnus . . 84

" Sporochnus . . . . 84

"Stilophora. . . . . 84

" paradoxus Sporochnus. 83

rhizophorum Ulonema . . . . 114

Rhodochorton . . 180, 211, 225

Rhododermis . . : . . 202

Rhododiscus . . . . . . 200

Rhodomela 161, 163, 172, 176, 225

Rhodomenia . 139, 144, 145, 146, 149

Rhodophyllis . . . 146, 225

Rhodophysema . . . . . 202

Rhodymenia 139, 141, 144, 146, 150

rhunensis Polysiphonia . . . 224

Rhynchococcus . . . . . 147 rigidula Polysiphonia . . . . 170

" Rytiphlœa . . . . . 176

" Sphacelaria. . . . . 99

rigidum Gelidium . . . . . 135

" Phycoseris . . . . . 62

" Ulva . . . . . . 61,62

rimosa Pilinia . . . . . . 63

riparia Acinetospora . . . . 112

" Rhizoclonium . . . . 67

Rivularia. . . 46, 49, 143, 228

Rivulariæ Elachista. . . . . 87

Rivulariarum Lyngbya. . . . 40

rivularis Enteromorpha . . . 58

" Palmella . . . . 51

robusta Enteromorpha . . . 60

"Polysiphonia. . . . 171

Rosenvingii Peyssonnelia. . . 202

roseolum Callithamnion . . . 177

" Polysiphonia . 171, 172

" Spermothamnion ... 177

roseus Actinococeus . . . . 143

" Callithamnion . . . 182, 185

". Chrysymenia . . . . 154

"Chylocladia . . . . . 154

" Conchocelis . . . . 73

" Contarinia . . . . . 201

" Cruoria . . . . . . 201

" Hapalidium . . . . . 205

" Hildenbrandia . . . 211

"Lomentaria . . . . 154

" Oscillatoria . . . . . 37

" Ostreobium . . . . . 73

" Phlebothamnion . . . 185

" Polysiphonia . . . . 165

" Rivularia. . . . . . 143

" Spirulina . . . . . . 34

" purpurea Cruoria . . . 201

" scopulorum Callithamnion 185

" tenue Callithamnion . . 185

rostellata Vaucheria . . . . 74

Rothiana Enteromorpha . . . 56

" gracilis Ulva. . . . 59

Rothii . . . . . . . 181

rotundus Polyides . . . . . 200

rubens Corallina . . . . . 210

"Delesseria . . . . . 141

" Hæmatocelis . . . . 202

" Halymenia . . . . 144

" Jania. . : . . . . 210

" Phyllophora . . . 141, 223 
rubra Calothrix. . . . . 45

" Ceramium . . . . . 194

" Hildenbrandia . . . . 211

Peyssonnelia . . . 202

Schizothrix . . . . . 45

Zonaria . . . . . 202

pedicellatum Ceramium . 193

tenue Ceramium . . . 195

" virgatum Ceramium . . 195

Rudolphiana Cladophora . . 72, 216

rufescens Schizosiphon . . . 45

rugosus Asperococcus . . . . 96

bullosus Asperococcus . 96

rupestris Cladophora . . . . 72

" Hydrocoleum . . 43, 219

" Peyssonnelia. . . . 202

" Phormidium . . . . 38

rupicola Callithamnion . . . 185

ruscifolium . . . . . . . 159

Rytiphloea . . 163, 170, 172, 176

saccharina Laminaria . . . 81, 220

Saccorhiza. . . . . . . 81

salina Dasyactis. . . . . . 46

"Hrmatococcus . . . . 52

" Microcoleus . . . . . 42

"Schizosiphon . . . . 46

" Scytonema . . . . . 45

"Vaucheria . . . . . 74

Sandrianum Aglaophyllum . . 158

" Ectocarpus . . 107, 218

Delesseria . . . 158

" Nitophyllum . . . 158

sanguinea . . . . . . . 158

Sareophyllis : . . . 145, 198

Sargassum . . . . . . .122

sarniensis Chondrus . . . . 138

Cladophora. . . . 69

Halymenia . . . 151

Rhodymenia . . . 151

saxatilis Sphacelaria . . . . 99

Schimmelmannia . . . . . 196

Schizosiphon . . . . . . 45, 46

Schizothrix . . . 43, 45, 225

Schizymenia . . . . 198, 199

Schmitziella . . . . . . 203

Schousboei Dermocarpa . . . 33

Griffithsia . . . 179

Hæmatocelis . . . 201

Ophidocladus . . . 175

Plumaria . . . 189
Schousboei Polysiphonia . . . 175

$\begin{array}{lll} & \text { Ptilota. • • . } 189 \\ & \text { Schimmelmannia } & 196 \\ \text { " } & \text { Thuretella. . . } 196\end{array}$

Scinaia. . . . . . . . 133

scutata Pringsheimia . . . . 54

scoparioides Sphacelaria . . 101

scoparium . . . . . . . . 101

scopulorum Callithamnion . . 185

" Calothrix . . 44, 45

" Ceramium . . . . 185

" Inactis . . . . 43

" Polysiphonia . . 169

" Schizosiphon. . 45

scorpioides Alsidium . . . 161

n Ascophyllum . . 119

$"$ Bostrychia . .161, 214

" Ectocarpus. . . 107

" Plocamium . . . 161

" Rhodomela . . . 161

scorpioideum Cystoclonium . . 146

scutulata Elachista . . . . . 90

Scytonema. . . . . . . . 45

Scytosiphon . . 57, 84, 91, 96, 97

secundatum Callithamnion 132, 183, 214

" Chantransia . . 132

" Ectocarpus. . . 105

" Polysiphonia. . . 168

secundus . . . . . . . . 112

" Ectocarpus . . . . 112

" Giffordia . . . 112, 219

secundiflora . . . . . . 179

seirospermum Callithamnion. . 188

Seirospora. . . . . .187, 225

semi-cristata Rytiphloea . . . 176

semi-nervis Cryptonemia . . . . 197

semi-nudum Callithamnion . . 180

semi-pennatum Callithamnion . 177

semi-plena Calothrix . . . 39, 41

Lyngbya. * . 39, 40, 221

senticosa Hutchinsia . . . . 168

sericea Cladophora. . . . . 72

" Conferva . . . . . 68

" Gelidium . . . . . 135

" Ptilota . . . . . . 188

" Vaucheria . . . . . 75

serpens Callithamnion. . . . 186

serratus Fucus . . . . . . 117

Sertularia . . . . . . 101 
sertularioides Polysiphonia 168, 169, 170,224

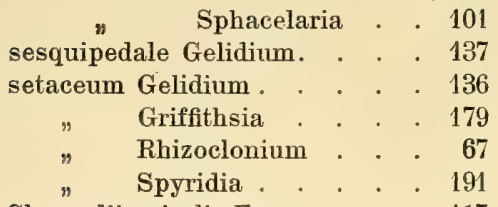

Sherardii spiralis Fucus . . . 117 silicidosus Eetocarpus . . . 103, 107

" firmus Ectocarpus. . 103

"uniformis Ectocarpus. 113

siliquosa Cystoseira . . . . 120

" Halidrys . . . . . 120

" Hormoceras . . . 195

simplex Callithamnion. . . 126, 186

" Desmarestia . . . . 92

" Dictyosiphon . . . . 91

" Eetocarpus . . . . 107

"Wildemania . . . . 129

simplicifilum Actinococeus . . 143

"Griffithsia . . . 179

"Halurus . . . 179

simplicipilum Spyridia. . . . 191

simpliciuscula Dasya . . . . 173

$\begin{array}{llll}" & \text { Ectocarpus } & . & 108 \\ " & \text { Ophidocladus. } & . & 175 \\ " & \text { Polysiphonia . } & . & 175 \\ " & \text { Sphacelaria } & . & 101\end{array}$

simulans Lithoderma. . . 80

" Melobesia. . . . 205

" Polysiphonia . . 169

$"$ Sorapion. . . . 80

sinuosa Colpomenia. . . . . 97

" Delesseria . . 158, 159, 217

" Encolium . . . . . 97

" Hydroclathrus. . . . 97

sirocladia Cladophora . . . . 68

Sirocoleum . . . . . . 43

smaragdinum Phormidium. . . 41

Smithii Nitophyllum. . . . . 157

sobolifera. . . . . . 151

Solenia. . . . . . . . 93

Solieria . . . . . . 147

Solierii Dasya . . . . . . 166

"Dictyota. . . . . . 125

" Spatoglossum . . . . 125

" Taonia . . . . . . 125

solitarium Streblonema . . . 110

sordida Lyngbya $\quad$. . . . 40, 221 sordida Ulva . . . . . . 55

Sorapion . . . . . . 80

Sorocarpus . . . . . . 113

Sowerbyanus Schizosiphon . . 45

spadiceum Phormidium . . . 41

sparsum Acrochætium. . . . 182

" Callithamnion . . . 182

" Rhodochorton . . . 182

spathulifera Corallina . . . 209, 210

Spatoglossum . . . . . . 125

speciosa Buffhamia. . . . 86

" Hormotrichum . . . 64

spectabilis Lyngbya $\ldots . \cdot \quad \cdot 221$

Spermatochnus . . . 83, 84, 225

Spermosira . . . . . . 50

Spermothamnion . . .177, 226

Sphacelaria . 86, 98, 101, 114, 226

Sphacelarioides Ectocarpus . . 108

" Giraudya . . 91

Sphacella . . . . . . . . 98

sphæricum Callithamnion . . 177

$"$ Ectocarpus . . 110

" Spermothamnion . 177

"Streblonema . . 110

Sphærococeus 135, 136, 137, 138, 139 , $140,141,142,143,144,145,146$,

$147,148,149,150,151,152,155$

sphærophorus Ascocyclus . . 111

Ectocarpus ․ 109

Isthmoplea . . 109

Sphærozyga . . . . . . . 49

Sphondylothamnion . .176, 226

spumigena Nodularia . . . 50

Spyridia . . . . . . . 190

spinella Dasya . . . . 173, 174

"Dasyopsis . . . . 174

spinescens Enteromorpha . . 60

spinosa Calliblepharis . . . . 149

" Callithamnion. . . . 187

" Hutchinsia . . . . 170

" Phlebothamnion . . 184

" Polysiphonia . . . 170

spinulosa Polysiphonia . . 169, 170

spiralis Dictyota . . . . . 123

Fucus . . 115, 116, 117, 118

$" \quad$ angustifolius Fucus . . 118

Spirulina . . . . . 34, 226

spongiocarpa Ralfsia . . . . 79

spongiosum Callithamnion . . 184

Cladostephus . 100, 101 
spongiosum Codium. . . . . 76

Spongodium . . . . . . 76

Spongomorpha . . . . . 70

Sporochnus $\mathbf{8 3}, 84,91,92,93,226$ squamaria Peyssonnelia . . . 202 squamata Corallina. . . . . 210 squarrosa Chylocladia. . . . 153

" Ectocarpus . . . 108

" Gastroclonium . . 153

" Lomentaria . . . . 153

squarrulosum Lithothamnion . 212

stellaris . . . . . . . . 90

stellatus Chondrus . . . . . 138

stéllulata Elachista. . . . . 90

" Myrionema . . . . 90

" Phycophila . . . . 90

Stenogramma. . . . . 141, 226

Sterrocolax . . . . . . . 143

stictiformis Melobesia . . . 205

Stictyosiphon. . . . .93, 103

stilla Cruoria. . . . . . . 201

Stilophora. . . . . . . 83, 84

Stilophoræ . . . . . . 110

stipitata Prasiola . . . . 54

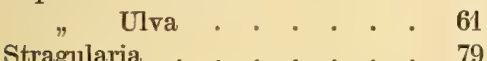

stragulum Lyngbya. . . . . 41

strangulans Myrionema . . . 87

strangulata Dermocarpa . . . 33

Streblocladia . . . . . . 165

Streblonema . . 109, 113, 226

Strepsithalia . . . . . 113

Striaria . . . . . . 84, 93

strictum Callithamnion . . . 177

" Ceramium. . . 194, 215

" Polysiphonia. . . 170

" Spermothamnion. . . 177

Stypocaulon . . . . 98, 101

Stypopodium . . . . . . . 79

subadunca intricata. . . . . 168

subæqualis Bangia . . . . 128

subarticulata Dasya . . . . 173

" Gastroclonium. . 153

" Phloeospora : . 93

" Stictyosiphon . . 93

subcontinua Polysiphonia. . . 170

subcostata . . . . . . . 133

subcutaneus . . . . . . . 143

subecostatus Fucus. . . . . 118

subdichotomum Lithophyllum . 205 subfuscum Phormidium . . . 38

" Rhodomela. . . 161, 225

submarina Palmella. . . . . 52

submembranaceum . . . . 38

subpectinata Cladophora . . . 72

subrefracta Cladophora . . . 67

subsalsa Spirulina . . . . . 35

subsimplex Lithothamnion . 206, 212

" Vaucheria. . . 75

subtenellum Lithophyllum. . . 212

subtilis Hormiscia . . . . . 62

" Plocamium . . . . 155

" Polysiphonia . . . 168

" Ulothrix. . . . . 62

subtilissima Sphacella . . . 98

" Spirulina. . . 35

subulatum Ceramium . . . . 171

" Ectocarpus . . . 103, 107

" Polysiphonia . . 171, 172

subulata major Grammita. . 171

subulifera . . . . . . . 170

subuliformis Oscillatoria . $35,36,37$

subvalidum Lithothamnion 206, 212, 220

subverticillatum . . . . . . 177

subviolacea Oscillatoria . . . 37

Sykidion . . . . . . . 53

Symploca . . . . . . . . 41

sympodicarpa Sphacelaria. . . 100

synandra Vaucheria * . . 75, 226

Taonia . . . . 124, 125

Teedii . . . . . . . 139

Tellamia . . . . . . . 64

tenella Chætomorpha . . . . 66

"Herposiphonia. . . 175

" Hutchinsia . . . . 175

" Polysiphonia . . . 169, 175

tenerrimus Microcoleus . . . 42

" Oscillatoria. . . . 35

tenue Ceramium . . . . . . 195

$"$ Enteromorpha . . . . 60

"Microcladia . . . . 195

$"$ Oscillatoria . . . . 37, 222

tenuissima Bangia . . . . 127

Bryopsis. . . . . 74

Callithamnion . . 188

Ceramium . . 188, 195

Chondria . . . 164

Chondriopsis . . 164

Dasya . . . . 174

Derbesia . . . 74 
tenuissima Enteromorpha . . . 60

Gigartina . . . . 164

Griffithsia * . . 178

Laurencia . . . 164

Nostoc . . . . . 49

Streblonema . . . 110

Polysiphonia . . 167, 172

Punctaria . . 95, 96

Rhodomela. . . 161

Seirospora . . . . 188

Spirulina . . . . 34

terebrans Plectonema. . . . 42

tergestina Oscillatoria. . . . 37

terminalis Ectocarpus . . . 108, 218

testarum Mastigocoleus . . 48, 221

tetragonum Callithamnion . 186, 214

Tetraspora . . . . . . . 94

tetricum Callithamnion . . . 186

Thamnidium . . . . . . 180, 181

thesiophylla Cystoseira . . . 122

thrix Chorda. . . . . . . 82

Thuretella. . . . . . . 196

Thuretii Acinetospora. . . . 112

"Choreonema . . . 203

" Melobesia. . . . 203

" Spirulina . . . . 35

" Vaucheria. . . 75, 227

thuyoides Callithamnion . . . 188

Ceramium . . . . 188

Polysiphonia . . 170

Rytiphlœea . . . . 170

Thwaitesii Anabæna . . . 49

Tilopteris . . . . . . . 114

tinctorium Phormidium . . . 36

" Rytiphloea . . . 176

tingitana Hutchinsia . . . . 166

tomentosa Chorda . . . . . 82

" Codium . . . 76, 216

" Ectocarpus . . . 108

tomentosum elongatum Codium. $\quad 76$

tomentosoides . . . . . . 111

torquescens Fucus . . . . . 118

Torreyi. . . . . . . . 142

torta Enteromorpha . . . . 60

tortilis Phlœospora. . . . . 95

tortuosa Chætomorpha . . . 66

Conferva . . . . . 66

Lithophyllum. . . . 206

Rhizoclonium. . . . 66

" crassum Lithophyllum. 206 torulosa Anabæna . . . . . 49

" Chætomorpha . . . 65

" Gastroclonium . . . 153

" Lomentaria . . . . 153

Tournefortiana Dictyota . . . 79

Trailliella . . . . . . . . 177

Tremella . . . . . . . . 51

Trentepohlia . . . . . . 132, 181

tribuloides Sphacelaria . . . 100

trichodes Enteromorpha . . . 57

tricuspidatum Gelidium . . 135

trifila Chantransia . . . . . 132

tripinnatum Callithamnion . 187, 188

Mertensia . . 187

Phlebothamnion . 187

tristis Bangia . . . . . 127

trochicola Calothrix . . . . 45

Schizosiphon . . 46

tuberculata Bifurcaria. . . . 119

Fucodium . . . . 119

Pyenophyeus . . 119

tuberculosa Castagnea. . . . 84

$" \quad$ Chordaria . . . 84

" Halorhiza . . . 84

" Stilophora. . . . 84

tuberiformis Leathesia. . . . 86

tubulosa Enteromorpha . . . 60

tumidus Choreocolax . . . . 133

Tuna . . . . . . . . 77

turgidus Chroococeus . . . 31

Turneri Asperococeus . . . . 96

Callithamnion . . . 177

Ceramium . . . . 177

Corynospora . . . . 177

Spermothamnion . . 177, 226 intricatum Spermothamnion . . . . . 177

Tylocarpus . . . . . . 142

typica Acinetospora . . . 112

$"$ Ectocarpus . . . . . 107

Ulex Sphacelaria . . . . 101

Ulonema . . . . . . 114

Ulothrix . . . . . . . 62,64

Ulva $55,56,57,58,59,60,95,128,129$

Ulvella. . . . . . . . 54

ulvoides Nitophyllum . . . 157

ulvoideum Nitophyllum . . 156

umbilicalis Porphyra . . . . 129

Ulva. . . . . . 128

Wildemania . . 129, 227 
umbilicalis lanceolata Ulva . . 129 umbilicata Peyssonnelia . . . 78 uncialis Spongomorpha . . . 70 uncinatus Ectocarpus . . . . 108

Enteromorpha . . . 56

Nitophyllum. . . 158, 222

Plocamium . . . 155

" Polysiphonia. . . . 168

undulatum Desmotrichum. . 96

Fucus . . . . 118

" Punctaria . . . . 96

uniforme Ceramium . . . . 195

unilaterale Callithamnion . . . 177

urceolata Polysiphonia. . . 170, 224

Urospora . . . . . . . . 64

ustulata . . . . . . . . 137

utriculosa Cladophora . . . . 72

uvaria . . . . . . . 155

uviformis Sorocarpus . . . 113

vadorum Conferva . . . . . 69

vaga Cladophora. . . . . . 72

"Halorhiza . . . . 84, 93

vaginata . . . . . . 4 43,225

Valiantei . . . . . . 111, 226

Valonia. . . . . . . $7 \boldsymbol{y}$

validum Lithothamnion . . . 212

Van Heurckii Epilithon • . . 207

Lithothamnion . . 207

"Rhododermis . . 202

variabilis Anabæna. . . . . 49

Callithamnion . . 178

variegata . . . . . . . . 171

Vaucheria . . . . . 74, 226

Vaucherii Chthonoblastus. . . 37

velutinus Ectocarpus . . . . . 86

" Elachista. . . . . 86

" Herponema . . . 86

" Sphacelaria . . . . 86

" Vaucheria . . . . 75

ventricosa Chrysymenia . . 155

" Dumontia . . . 155

" Enteromorpha. . . 58

venulosum Nitophyllum . . . 158

venusta Dasya . . . . . . 173

vermicellifera Porphyra . . . 128

vermicularis Asperococcus . . 96

" Calosiphonia . . 199

" Mesogloia . . . 88

" Nemastoma . . . 199

vermiculata Helminthocladia . 85 vermiculata Mesogloia . . . 85

vernale Gastroclonium. . . . 153

verrucata Melobesia. . . . 204, 212

verruciformis Janezewskia. . . 175

verrucosum Nostoc . . . . . 49

Ralfsia. . . . 80

verruculosa Amphiroa . . . 210

versicolor Aglaophyllum . . . 158

Bangia . . . 128, 214

Callithamnion 183, 188, 225

Lyngbya . . . . . 39

Lithophyllum . . . 158

Spirulina . . . 35, 226

seirospermum Calli-

thamnion . . . 188

vertebrata Erythrotrichia . . . 126

verticillatus Cladostephus. . . 101

" Dasya . . . . 174

" Gastroclonium . . 153

" Lophothalia . . . 174

vesiculosa Entophysalis . . . 33

" Fucus . . . .115, 117

" Placoma . . . . 31

" angustifolius Fucus . 118

" axillaris Fucus . . 115

" contortus Fucus . . 117

" evesiculosus Fucus . 116

" foliaceus Fucus . . 118

" globosus Fucus . . 118

" inflatus Fucus . . 118

"linearis Fucus . . 118

" longifructus Fucus . 118

" lutarius Fucus. . . 115

" monocystus Fucus : 115

" Sherardii Fucus . . 116

" spiralis Fucus . . 117, 118

Vidalia. . . . . . . 176

Vidovichii Callithamnion. . . 180

"Naccaria . . . . 134

villosa Arthrocladia. . . . . 93

" Sporochnus . . . . . 93

" Spyridia . . . . . . 191

" australis Arthrocladia . 93

villosissima Spyridia . . . . 190

villosiuscula Spyridia . . . . 191

vimineum Ceramium . . . . 195

violacea Amphithrix . . . . 43

Dermocarpa . . . 33

Hypheothrix . . . 43

Leibleinia . . . . 40 
violacea Lyngbya . . . . . 40

" Polysiphonia . 166, 171, 172

" fibrillosa Polysiphonia . 166

" tenuissima Polysiphonia 172

violascens Polysiphonia . . . 168

virens Polysiphonia. . . . . 175

virescens Ectocarpus . . . 218,108

Eudesme . . . . . 87

" Myriocladia . . . . 87

virgata Corallina. . . . . . 209

"Fucus. . . . . . . 118

virgatulum . . . . . . . 132

viridis Demarestia . . . . . 92

" Ectocarpus . . . . . 107

"Endoderma . . . . . 63

" Halosphæra . . . . 53, 219

" Lyngbya . . . . . 40, 221

" Oscillatoria . . . . . 37

"Sporochnus . . . . . 92

viscida Liagora . . . . . 131, 133

volubilis Cylindrocarpus . . . 111

" Dictyomenia . . . . 176

" Dictyota .. . . . 123

" Ectocarpus . . . . 111

" Fucus . . . . . . 118

" Rhodomela . . . 176

" Streblonema - 111, 109226

"Vidalia . . . . . 176

voluticola Hyella . . . . . 34

Volvox'. . . . . . . . . 52

vulgaris Dictyota . . . . 123

" Fucus . . . . . . 118

" Myrionema . . . 87

" Oscillatoria. . . . 37 vulgare Plocamium. . . 154,147

" Porphyra . . . . 128, 129

" Sargassum. . . . . 122

n publica Oscillatoria . . 37

Warrenii Schizosiphon. . . . 46

Welwitschii Erythrotrichia . . 127

Westendorpii Fucus. . . . . 118

Wigghii . . . . . .134, 222

Wildemania . . . 128, 227

Wittrockii Endoderma. . . . 63

Monostroma . . . 56

Woodwardii Hypoglossum 159, 219

Wormskjoldia . . . . . 158

Wrangelia. . . . . 174, 176

Wulfenii Polysiphonia . . . 167

Zanardinia . . . . . . 78

Zanardinii Ectocarpus . . . 111

"Spirulina . . . . 34

"Streblonema . . 111

zonalis Melobesia . . . . . 204

Zonaria $78,79,80,93,95,123,202$

zonata Dictyota. . . . . 124

"Isactis . . . . . . 46

Zonotrichia . . . . . . . 47

Zosteræ Castagnea. . . . . 87

"Mesogloia . . . 85, 87

" Myriocladia . . . . 85

zostericola Calothrix . . . . 44

" Ceramium . . 194, 215

" Oscillatoria. . . 37

" Seytosiphon . . . 97

zosterifolia Phyllitis . . . . 97

Zygomitus. . . . . . . 64 


\section{TABLE DES MATIÈRES}

Sources des renseignements.

PAGE

Laboratoires maritimes. . . . . . . . . . . . . . 1

Mes herborisations . . . . . . . . . . . . . . . 1

Épaves . . . . . . . . . . . . . . . . . . . 2

Profondeurs . . . . . . . . . . . . . . . . . . 2

Les herbiers . . . . . . . . . . . . . . . . . . 3

Les livres. . . . . . . . . . . . . . . . . . 4

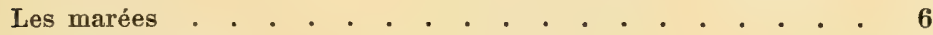

Champ d'exploration . . . . . . . . . . . . . . . . 8

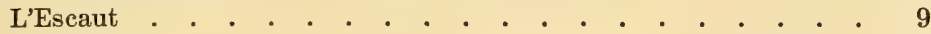

La côte belge. . . . . . . . . . . . . . . . . . 9

De Wimereux au Havre . . . . . . . . . . . . . . 10

Tatihou . . . . . . . . . . . . . . . . . . . . 11

Cherbourg . • . . . . . . . . . . . . . . . . 11

Iles normandes . . . . . . . . . . . . . . . . 12

Ile Bréhat . . . . . . . . . . . . . . . . . . 13

Carantec, île Callot, le Cerf . . . . . . . . . . . 13

Roscoff . . . . . . . . . . . . . . . . . 13

Brest et les environs . . . . . . . . . . . . . 17

La presqu'île de Crozon . . . . . . . . . . . . . 18

Belle-Isle . . . . . . . . . . . . . . . . . . . 19

Le Morbihan . . . . . . . . . . . . . . . . . 19

Le Croisic . . . . . . . . . . . . . . . . . . . 19

Le bassin d'Arcachon . . . . . . . . . . . . . 20

Biarritz . . . . . . . . . . . . . . . . . . 20

De Biarritz à Guéthary. . . . . . . . . . . . . . . 22

St. Jean de Luz, Socoa, Ste-Barbe . . . . . . . . . . . 23

Rochers Ste-Anne, Andagorria . . . . . . . . . . . . 24

Le cap Figuier . . . . . . . . . . . . . . . . . 24

La côte S. du golfe de Gascogne . . . . . . . . . . . 25

Améliorations futures de la Liste . . . . . . . . . . . . . 26

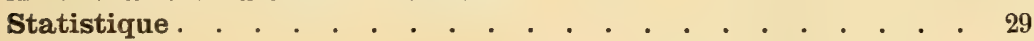

Abréviations . . . . . . . . . . . . . . . . . . . 30

\section{Ordre des Myxophycées}

Coccogonées.

Chroococcacées . . . . . . . . . . . . . . . 31

Chamésiphonées . . . . . . . . . . . . . . . . 32

Hormogonées.

Homéocystées ou Oscillariées.

Lyngbyées . . . . . . . . . . . . . . . . . . . 34

Vaginariées. . . . . . . . . . . . . . 42 
Hétérocystées ou Nostochinées.

PAGE

Rivulariées.

Sirosiphonées. . . . . . . . . . . . . . . 48

Scytonémées . . . . . . . . . . . . . . . . . . . 48

Nostocacées . . . . . . . . . . . . . . . 49

\section{Ordre des Chlorophycées}

Protococcacées . . . . . . . . . . . . . . . . . 51

Confervacées. . . . . . . . . . . . . . . . . . . . . 53

Siphonées. . . . . . . . . . . . . . . . . 73

\section{Ordre des Phéophycées}

Phéosporées.

Cutlériacées . . . . . . . . . . . . . . . . . 78

Ralfsiées . . . . . . . . . . . . . . . . . . 79

Laminariées . . . . . . . . . . . . . ... . 80

Sporochnées . . . . . . . . . . . . . . . 83

Chordariées . . . . . . . . . . . . . . 84

Elachistées. . . . . . . . . . . . . . . . . . . 89

Ponctariées. . . . . . . . . . . . . . . 91

Encéliacées. . . . . . . . . . . . . . . . . 94

Phéocapsées . . . . . . . . . . . . . . 9 94

Sphacélariées . . . . . . . . . . . . . . . 98

Ectocarpées . . . . . . . . . . . . . . . . 102

Tiloptéridées . . . . . . . . . . . . . . . . . . 114

Fucacées . . . . . . . . . . . . . . . . . . . 115

Ordre des Dictyotées . . . . . . . . . . . . . . . . . 123

\section{Ordre des Rhodophycées ou Floridées}

Porphyrées ou Bangiales. . . . . . . . . . . . . . . 126 Eufloridées.

Némalionales.

Helminthocladiées . . . . . . . . . . . . . . 129

Chétangiées . . . . . . . . . . . . . . 133

Gélidiacées. . . . . . . . . . . . . . . . 134

Gigartinales.

Gigartinées. . . . . . . . . . . . . . . . 137

Rhodophyllidées . . . . . . . . . . . . . . . 145

Rhodyméniales.

Sphérococcacées . . . . . . . . . . . . . . 147

Rhodyméniées. . . . . . . . . . . . . . 150

Delessériées . . . . . . . . . . . . . . . 156

Bonnemaisoniées. . . . . . . . . . . . . . 160

Rhodomélées . . . . . . . . . . . . . . . . 161

Céramiées . . . . . . . . . . . . 176 
Cryptonémiales. PAGE

Gléosiphoniées . . . . . . . . . . . . . 195

Grateloupiacées . . . . . . . . . . . . 196

Dumontiacées . . . . . . . . . . . . . . . . . 198

Némastomacées . . . . . . . . . . . . . . . 199

Rhizophyllidées . . . . . . . . . . . . . 200

Squamariées . . . . . . . . . . . . . . . 200

Corallinées. . . . . . . . . . . . . . . . 203

Appendice des Gorallinées . . . . . . . . . . . . . . . . 212

Florule de Tatihou. . . . . . . . . . . . . . . . . . 213

Errata . . . . . . . . . . . . . . . . . . 228

Répertoire alphabétique général . . . . . . . . . . . 229

Table des Matières. . . . . . . . . . . . . . . . . . 257 

EN VOIE D'ACHÈVEMENT

ET POUR PARAITRE SUCCESSIVEMENT

\section{PRODROME DE LA FLORE}

DES

\section{ALGUES MARINES}

DES ILES ANGLO-NORMANDES ET DU NORD-OUEST DE LA FRANCE

PAR LE

Dr HeNRI VAN HEURCK

PROFESSEUR-DIRECTEUR AU JARDIN BOTANIQUUE D'ANVERS

LES DIATOMÉES

DE L'EXPÉDITION ANTARCTIQUE

DE LA

\section{BELGICA}

GRAND IN $-4^{\circ}$ AVEC ENVIRON 300 FIGURES DE FORMES CRTTIQUES OU NOUVELLES

PAR IE MÂME

\section{LE MICROSCOPE}

SA CONSTRUCTION, SON MANIEMENT ETC.

PAR LE MÊME

6e ÉDITION

GRAND IN-8 $8^{\circ}$ AVEC NOMBRLUSES FIGURES. 